\title{
Structural and optical impact of transition metal implantation into zinc oxide single crystals and nanowires
}

Dissertation

zur Erlangung des Doktorgrades

der Mathematisch-Naturwissenschaftlichen Fakultäten der Georg-August-Universität zu Göttingen

\author{
vorgelegt von \\ Sven Müller \\ aus
}

Eilenburg

Göttingen, 2009 
D7

Referent:

Prof. Dr. C. Ronning

Koreferent:

Prof. Dr. R.G. Ulbrich

Tag der Mündlichen Prüfung: 30. März 2009 


\section{Contents}

$\begin{array}{llr}1 & \text { Introduction } & 7\end{array}$

2 Transition metals in semiconductors $\quad 13$

2.1 Magnetism . . . . . . . . . . . . . . . . . . . . . 16

2.1.1 Direct and super-exchange . . . . . . . . . . . 16

2.1.2 Carrier-mediated exchange . . . . . . . . . . . . 16

2.1.3 Double exchange . . . . . . . . . . . . . . 18

2.1.4 Bound magnetic polaron . . . . . . . . . . . . . . . 20

$2.23 d$-shell degeneration . . . . . . . . . . . . . . . . . . . 21

2.2.1 Crystal field perturbation . . . . . . . . . . . . . . 22

2.2.2 Spin-orbit interaction . . . . . . . . . . . . . 26

2.2 .3 Kramers degeneracy . . . . . . . . . . . . . . . 27

2.2.4 Jahn-Teller distortion . . . . . . . . . . . . . . . . . . . . . 27

2.3 Band-gap . . . . . . . . . . . . . . . . . . . 30

3 Preparation of transition metal alloyed $\mathrm{ZnO} 33$

3.1 Bulk crystals . . . . . . . . . . . . . . . . . 33

3.2 Thin films . . . . . . . . . . . . . . . . . 36

3.2.1 Pulsed laser deposition . . . . . . . . . . . . . . 36

3.2.2 Sputtering . . . . . . . . . . . . . . 37

3.2 .3 Sol-gel process . . . . . . . . . . . . . . . . . . 38

3.3 Nanowires . . . . . . . . . . . . . . . . . . . . . . 39

3.3.1 Vapor-liquid-solid process . . . . . . . . . . . . 39

3.3.2 High temperature growth of $\mathrm{ZnO}$ nanowires . . . . . . . . . . 45

3.4 Ion implantation and annealing . . . . . . . . . . . . . 47

4 Characterization techniques $\quad 51$

4.1 Electron microscopy . . . . . . . . . . . . . . . . 51

4.2 X-ray diffraction . . . . . . . . . . . . . . . . 54 
4.3 Raman spectroscopy . . . . . . . . . . . . . . . . . 55

4.4 Photo- and cathodoluminescence . . . . . . . . . . . . . . 58

4.5 The measurement of color . . . . . . . . . . . . . . . . . . . . 60

4.6 Deep-level transient spectroscopy . . . . . . . . . . . . . . . 61

5 Structural impact of transition metal implantation into $\mathrm{ZnO} \quad 63$

5.1 Crystal properties of pure $\mathrm{ZnO} \ldots \ldots . \ldots 63$

5.2 Situation after ion implantation . . . . . . . . . . . . . . 69

5.3 Annealing of defects . . . . . . . . . . . . . . . . . 72

5.4 Local vibration modes of manganese in $\mathrm{ZnO}$. . . . . . . . . . . 78

5.5 Secondary phase formation . . . . . . . . . . . . . . . . . . . 81

5.5.1 $\mathrm{ZnMn}_{2} \mathrm{O}_{4}$ in $\mathrm{Mn}$ implanted $\mathrm{ZnO} \ldots \ldots . . . . . . . .883$

5.5.2 $\mathrm{ZnCo}_{2} \mathrm{O}_{4}$ and $\mathrm{CoO}$ in $\mathrm{Co}$ implanted $\mathrm{ZnO}$. . . . . . . . . . . 89

5.5.3 $\mathrm{ZnFe}_{2} \mathrm{O}_{4}$ in $\mathrm{Fe}$ implanted $\mathrm{ZnO}$. . . . . . . . . . . . . . . . . 94

5.5.4 Formation of $\mathrm{NiO}$ secondary phases . . . . . . . . . . . . 98

5.6 Summary . . . . . . . . . . . . . . . . . . . . . . . . . 101

6 Luminescence of transition metal implanted $\mathrm{ZnO} 105$

6.1 The shallow indium donor . . . . . . . . . . . . . . . . . . . 109

6.2 Situation after transition metal implantation . . . . . . . . . . . . . 111

6.3 Deep center luminescence . . . . . . . . . . . . . . . . . . . . 112

6.3.1 The oxygen vacancy in $\mathrm{ZnO}$. . . . . . . . . . . . . 113

6.3.2 Red-yellow luminescence band in $\mathrm{ZnO}$. . . . . . . . . . . . 116

6.3.3 Dingle model . . . . . . . . . . . . . . . . . . . 119

6.3.4 Configuration coordinate model for the structured green luminescence band of $\mathrm{ZnO}$. . . . . . . . . . . . . . . 120

6.3.5 Interstitial zinc and oxygen in the green luminescence band . . 124

6.4 Intra-shell luminescence of transition metals . . . . . . . . . . . . 126

6.4.1 $\mathrm{Co}^{2+}$ ion . . . . . . . . . . . . . . . 126

$6.4 .2 \mathrm{Fe}^{3+}$ ion . . . . . . . . . . . . . . . . . 132

6.4.3 $\mathrm{Ni}$ and $\mathrm{Mn}$ ions in $\mathrm{ZnO}$. . . . . . . . . . . . . . . . . 140

6.5 White color luminescence emission of $\mathrm{V}$ implanted ZnO PLD films . . 142

6.6 Band gap shift in $\mathrm{ZnO}: \mathrm{Mn}$. . . . . . . . . . . . . . . . . . . . . 145

6.7 Summary . . . . . . . . . . . . . . . . . . . . . . . 146

7 Summary and Outlook $\quad 149$

7.1 Structural impact of ion implantation onto single crystals . . . . . . . 149

7.2 Secondary phases . . . . . . . . . . . . . . . . . 150 
7.3 Defect luminescence . . . . . . . . . . . . . . . . . . . . . . . . 150

7.4 Intra-shell luminescence . . . . . . . . . . . . . . . . . . . . 151

7.5 Transition metal implanted $\mathrm{ZnO}$ nanowires . . . . . . . . . . . . . . . 152

$\begin{array}{ll}\text { List of Figures } & 153\end{array}$

$\begin{array}{ll}\text { List of Tables } & 157\end{array}$

$\begin{array}{lr}\text { Bibliography } & 159\end{array}$

$\begin{array}{lr}\text { A Publications } & 173\end{array}$ 


\section{Chapter 1}

\section{Introduction}

Zinc oxide $(\mathrm{ZnO})$ is a II-VI wide band gap semiconductor $(3.437 \mathrm{eV})$ with an exciton binding energy of $60 \mathrm{meV}$ [1], causing stable excitons at room temperature, and therefore it is an extremely promising candidate for efficient visible-UVoptoelectronics [2]. Furthermore, $\mathrm{ZnO}$ as well as GaN are the most promising candidates for spin-based electronic devices, since room temperature ferromagnetism was predicted for transition metal alloyed $\mathrm{ZnO}$ and $\mathrm{GaN}$ [3-5]. The key question for the theoretical descriptions of the ferromagnetic origin in diluted magnetic semiconductors is the electronic configuration of the transition metal $3 d$-shell and its host lattice interaction to provide a spin-spin exchange mechanism between the magnetic impurities. There are three popular spin-coupling mechanisms: carrier exchange [3], double exchange [5-7], and coupling via a bound magnetic polaron [8-10]. Therefore, the charge state and the involved electronic $3 d$-shell configuration of transition metal ions, as well as the nature of defects in the host crystal are crucial points in understanding the magnetic mechanism within the diluted magnetic semiconductors. However, experimental data reveal inconsistent magnetic properties for transition metal alloyed $\mathrm{ZnO}[6 ; 8 ; 11-13]$, leaving the real mechanism for ferromagnetism in $\mathrm{ZnO}$ unclear. On the other hand, the formation of transition metal rich segregations in transition metal alloyed $\mathrm{ZnO}$ have a major effect onto the magnetic properties [14-20].

The incorporation of transition metal centers in wide band gap semiconductors results in a multiple splitting of degenerated $3 d$-shell states of free transition metal ions. The electronic structure is affected by the Stark effect of the crystal field, which has trigonal symmetry $\left(\mathrm{C}_{3 \nu}\right)$ in hexagonal wurtzite structures like $\mathrm{ZnO}$ and is treated as perturbation of a tetrahedral $\left(\mathrm{T}_{d}\right)$ symmetry of a cubic crystal $[21 ; 22]$. Further splitting of the electronic structure results from spin-orbit interaction, vibra- 
tional contributions and Jahn-Teller coupling [23]. As a result, transitions between these $3 d$-shell state become partly allowed and show very sharp transitions with long lifetimes (up to milliseconds) [22-25]. In addition to spintronic applications, these intra-shell transitions could be used in optoelectronic devices (light-emitting diodes or laser diodes). E.g. such intra-shell transitions of ions are commonly used in conventional optical communication technology for generation, guiding, switching, and amplification of light [26]. Microelectronic industry intends to use these concepts for optical on- and off-chip communication to conquer limitations of electronic communication [27-29].

The bottom-up production process of one-dimensional (1D) semiconductor nanostructures, especially nanowires, offers a great chance to overcome the limitations of up-to-date microelectronic devices built by top-down lithography processes. Up to now, field effect transistor arrays, logical and computational gates [30], nanosized light-emitting diodes and detectors [31-33], and nanosensors with functionalized semiconductor surfaces [34;35] have been demonstrated on the basis of semiconductor nanowires. $\mathrm{ZnO}$ nanowires can be applied in light-emitting diodes (LED), lasers or waveguides [36-38]. Commonly $\mathrm{ZnO}$ nanowires are grown via the vapor-liquid-solid growth mechanism [39; 40], which was already described in the 1960s for gold-assisted growth of silicon whiskers [41]. The utilization of transition metal alloyed $\mathrm{ZnO}$ nanowires for LEDs or spintronics requires the control of the transition metal concentration and distribution across the whole nanowire.

Typically, $\mathrm{ZnO}$ nanowires are grown by vapor transport with a source temperature above $800^{\circ} \mathrm{C}[39 ; 40]$. These high temperatures and the common introduction of oxygen are crucial parameters for the formation of $\mathrm{Zn}_{1-x} \mathrm{TM}_{x} \mathrm{O}$ nanostructures [42-45]. A too high temperature and oxygen partial pressure would force the oxidation of the transition metal in the source or nanowires and lead to a reduced transition metal concentration or the formation of possible transition metal oxide secondary phases within the nanowires. However, ion implantation of transition metals into semiconductor nanowires allows the direct control of the concentration and distribution, and is a common tool to avoid the limitations during growth [46-48].

The major disadvantage of the ion implantation is the formation of numerous defects, which requires an additional annealing step. Annealing of transition metal implanted $\mathrm{ZnO}$ thin films and nanowires in air or oxygen leads to the formation of a broad luminescence band in the visible spectral region [49; 50]. An almost whitish photoluminescence of those samples was shown, revealing the potential of $\mathrm{ZnO}$ for white light emitting diodes [49].

Light emitting diodes (LED) exist for the blue, green, yellow, and red spectral 
range of visible light [51]. They are either based on band-gap engineered ternary III-V semiconductors like InGaN, GaAlAs, or AlInGaP $[52 ; 53]$ or on wide band gap semiconductors (c-BN, AlN, GaN, ZnO, or ZnS) doped with color centers like rare earth elements or transition metals [54-58]. Such LEDs are used for lasers or for one color applications because of their high energy conversion efficiency. Also for illumination purposes white light diodes are envisioned in order to replace light bulbs and fluorescent lamps to reduce the power consumption of illuminants [59; 60]. White light sources on the basis of LEDs are usually fabricated in two different ways. The integration of red, green, and blue LEDs enables independent control of the three different color intensities yielding full-color displays [52]. The other route includes the use of near-ultraviolet light-emitting InGaN to excite a blue/yellow phosphorus [61]. Both approaches are effective, but reduce the energy conversion efficiency, are costly and complicated due to the multiple processing steps needed for the realization of white light emission.

White light emitting diodes were realized via a multilayer heterostructure consisting of $\mathrm{n}-\mathrm{ZnO}: \mathrm{In} / \mathrm{p}-\mathrm{GaN}: \mathrm{Mg} / \mathrm{n}-\mathrm{GaN}$ on sapphire, where white electroluminescence was generated at the $\mathrm{n}-\mathrm{ZnO} / \mathrm{p}-\mathrm{GaN}$ interface [62]. There, the white light is generated due to recombinations involving deep defects in the active region and strongly depends on defects generated during the fabrication of the multilayer heterostructure. Defects, which lie deep in the band gap of a semiconductor, behave as strong recombination centers (for a detailed list of deep defect centers in $\mathrm{ZnO}$ see Ref. [63] and references therein). For example, the broad green and yellow luminescence bands with modulated structure on the high energy side in $\mathrm{ZnO}$ and GaN, respectively, are due to the recombination between shallow donor levels and mainly the ground state of a deep level plus an integral number of a principal optical phonon energy $[64 ; 65]$. If distinct emission bands in the blue, green, and red spectral ranges are generated by defect-related donor-acceptor pair (DAP) transitions, the combined emission bands could give a spectrum that appears white to the naked eye.

It is quite complicated to produce a $\mathrm{ZnO}$-based pn-diode [66-68]. Among the potential impurities acting as acceptors, the group-V elements nitrogen, phosphorus, arsenic, and antimony are the most promising [69]. However, the realization and engineering of devices require both n- and p-type dopings as well as detailed knowledge of the doping issues, such as carrier concentration, mobilities, doping species, doping levels, and solubility limits. ZnO crystals are intrinsically n-type conducting due to native defects such as oxygen vacancies $\left(\mathrm{V}_{O}\right)[70 ; 71]$, zinc interstitials $\left(\mathrm{Zn}_{I}\right)$ [72], or donor impurities [73-75]. Therefore, the luminescence of $\mathrm{ZnO}$ shows a variety of very sharp lines in the photoluminescence spectra in the near band gap region. 
Most of these intense luminescence lines are usually dominated by transitions of donor bound excitons, which are commonly labeled from $\mathrm{I}_{1}$ to $\mathrm{I}_{11}$ [73], whereby the identity of the corresponding donors is in the most cases unknown. Some of them were assigned to specific elements such as hydrogen, aluminum, or gallium [73; 74].

The aim of this work is the controlled incorporation of transition metals into $\mathrm{ZnO}$ nanowires via ion implantation and the successful activation of the ion implanted transition metal ions in the $\mathrm{ZnO}$ host matrix. The results of the transition metal implanted $\mathrm{ZnO}$ nanowires are compared with observations of transition metal implanted bulk $\mathrm{ZnO}$ single crystals.

The successful activation of the transition metal ions in $\mathrm{ZnO}$ is determined via luminescence measurements (photo- and cathodoluminescence). Furthermore, the annealing effect of the implantation induced defects is studied by Raman spectroscopy and luminescence spectroscopy. However, broad deep luminescence bands are observed after ion implantation and annealing, the chemical nature of those bands will be discussed within this work. High dose transition metal implanted ZnO single crystals show the formation of secondary phases after annealing, which are determined as far as possible by means of Raman spectroscopy, X-Ray diffraction and transmission electron microscopy.

An introduction to the physical properties of transition metals in semiconductors is given in chapter 2 with focus on $\mathrm{ZnO}$. The prominent spin-coupling mechanisms are described including the theoretical predictions of the magnetic ordering in transition metal alloyed $\mathrm{ZnO}$. On the other hand, an introduction to the $3 d$-shell degeneration is presented.

Chapter 3 presents the preparation techniques of transition metal alloyed ZnO. Not only the preparation of $\mathrm{ZnO}$ nanowires and the issues to prepare transition metal alloyed $\mathrm{ZnO}$ nanowires are discussed, the chapter starts with the fabrication of bulk $\mathrm{ZnO}$ crystals and thin films. Within this chapter the growth of the CrysTec single crystals, pulsed laser deposited thin films as well as ZnO nanowires are shown, which are ion implanted with transition metal ions.

In chapter 4 the used characterization techniques are described, including the definition of color.

The structural properties of transition metal implanted $\mathrm{ZnO}$ directly after the ion implantation process as well as the annealing effect is presented in chapter 5 . The observation of a possible local vibration mode of $\mathrm{Mn}$ on a zinc lattice side is 
discussed in this chapter. The chapter winds up with the discussion and delimitation of secondary phases in high dose transition metal implanted $\mathrm{ZnO}$.

In chapter 6 the luminescence properties of transition metal implanted $\mathrm{ZnO}$ are presented and discussed. The chemical nature of the photoluminescence $\mathrm{I}_{9}$ line in the near band gap luminescence of $\mathrm{ZnO}$ is assigned to a shallow indium donor by intensity decay study of the $\mathrm{I}_{9}$ line after ion implantation of the radioactive ${ }^{111} \mathrm{In}$ isotope. The ion implantation of transition metals into $\mathrm{ZnO}$ as well as the subsequent annealing in air or oxygen results in broad and deep luminescence bands in the visible range. The chemical nature of the corresponding defects is discussed in the first major part of this chapter. The second major part discusses the observed intrashell luminescence of the transition metal ions in $\mathrm{ZnO}$, including the luminescence properties at different temperatures and excitation intensities.

The last chapter gives a short summary of this thesis and presents a short outlook for future work. 


\section{Chapter 2}

\section{Transition metals in semiconductors}

Diluted magnetic semiconductors (DMS) are semiconductive materials where a fraction of the host cations are replaced by magnetic ions such as transition metals (TM) or rare earth elements. DMS materials show a potential for spintronic devices which utilize the spin in magnetic materials together with the charge of electrons in semiconductors $[11 ; 13 ; 76 ; 77]$. For practical applications in spintronic devices, it is obvious that DMS materials have to show ferromagnetism with a Curie temperature above room temperature. In the early period especially $\mathrm{A}^{I I} \mathrm{~B}^{V I}$ compounds (with $\mathrm{A}=\mathrm{Zn}, \mathrm{Cd}$ and $\mathrm{B}=\mathrm{S}, \mathrm{Se}, \mathrm{Te}$ ) alloyed with transition metals ions were studied, but almost all show paramagnetic, antiferromagnetic or spin-glass behavior [78; 79]. The breakthrough was achieved with Mn alloyed III-V semiconductors, such as the 'high temperature' ferromagnetic GaMnAs. First observations of holemediated ferromagnetism (carrier-mediated exchange) in GaMnAs exhibit a Curie temperature of $110 \mathrm{~K}$ [80], which was increased to $173 \mathrm{~K}$ by MBE growth [81; 82]. Adapted from this experimental observation, Dietl et al. predicted ferromagnetism in $\mathrm{Mn}$ alloyed $\mathrm{ZnO}$ and $\mathrm{GaN}$ with Curie temperatures above room temperature [3], if both materials are highly p-type doped.

Two other popular spin-coupling mechanisms used to describe ferromagnetic ordering within semiconductors are: double exchange $[5 ; 7 ; 11]$, and coupling via a bound magnetic polaron [8-10]. The carrier exchange interaction requires free carriers for the spin coupling between TM centers [3], where a large number of free valence band or conduction band carriers and levels close to the bands is necessary. The Zener double exchange is an indirect coupling mechanism between two neighboring TM centers in different charge states, where the electron exchange is mediated 
by an oxygen atom [7]. The electron is transferred from TM ions in low states to TM ions in higher states if both magnetic moments are aligned parallel [7]. Spin coupling via a bound magnetic polaron is induced by alignment of the polaron spin to all impurities with a half-full or more than half-full $3 \mathrm{~d}$-shell within the hydrogen polaron orbit [9]. Therefore, the charge state and the involved electronic 3d-shell configuration of TM ions are crucial points in understanding the magnetic mechanism within the diluted magnetic semiconductors. Typically, all transition metal atoms occupy a zinc lattice sides in $\mathrm{ZnO}[83 ; 84]$. Given that zinc is commonly double positive charged within the $\mathrm{ZnO}$ lattice, all TM ions on a zinc lattice side are double positive charged ions in $\mathrm{ZnO}$ : $\mathrm{TM}^{2+}$. Table 2.1 shows the electronic configuration of transition metals with a completely filled 4s-orbital with the exception of $\mathrm{Cr}$ [85]. The d-shell of transition metals has ten available states, thereby the $3 \mathrm{~d}$ band of $\mathrm{Mn}^{2+}$ and $\mathrm{Cr}^{2+}$ is half-filled with 5 electrons with up-spin. The electronic configuration of the $3 \mathrm{~d}$-shell of a transition metal ion is commonly indicated with $\mathrm{d}^{n}$ (with $n$ the number of electrons in the d-shell). Therefore, the $\mathrm{Mn}^{2+}$ ion has a

Table 2.1: Electronic configuration of the 3d-shell of transition metal ions in crystal fields [85]. At this point further perturbations by the crystal field are neglected and discussed in section 2.2. Therefore, the last row specifies the ground state term of the corresponding free ion.

\begin{tabular}{|c|c|c|c|c|c|c|c|c|c|c|}
\hline \multicolumn{2}{|c|}{$\begin{array}{l}\text { Free transition } \\
\text { metal ion }\end{array}$} & \multicolumn{9}{|c|}{ Transition metal ions in crystal fields } \\
\hline $\mathrm{Ni}$ & $3 \mathrm{~d}^{8} 4 \mathrm{~s}^{2}$ & & & & & & & $\mathrm{Ni}^{4+}$ & $\mathrm{Ni}^{3+}$ & $\mathrm{Ni}^{2+}$ \\
\hline $\mathrm{Co}$ & $3 \mathrm{~d}^{7} 4 \mathrm{~s}^{2}$ & & & & & & & $\mathrm{Co}^{3+}$ & $\mathrm{Co}^{2+}$ & $\mathrm{Co}^{+}$ \\
\hline $\mathrm{Fe}$ & $3 \mathrm{~d}^{6} 4 \mathrm{~s}^{2}$ & & & & & & $\mathrm{Fe}^{3+}$ & $\mathrm{Fe}^{2+}$ & $\mathrm{Fe}^{+}$ & $\mathrm{Fe}^{0}$ \\
\hline $\mathrm{Mn}$ & $3 \mathrm{~d}^{5} 4 \mathrm{~s}^{2}$ & $\mathrm{Mn}^{7+}$ & $\mathrm{Mn}^{6+}$ & $\mathrm{Mn}^{5+}$ & $\mathrm{Mn}^{4+}$ & $\mathrm{Mn}^{3+}$ & $\mathrm{Mn}^{2+}$ & $\mathrm{Mn}^{+}$ & $\mathrm{Mn}^{0}$ & $\mathrm{Mn}^{-}$ \\
\hline $\mathrm{Cr}$ & $3 \mathrm{~d}^{5} 4 \mathrm{~s}^{1}$ & $\mathrm{Cr}^{6+}$ & $\mathrm{Cr}^{5+}$ & $\mathrm{Cr}^{4+}$ & $\mathrm{Cr}^{3+}$ & $\mathrm{Cr}^{2+}$ & $\mathrm{Cr}^{+}$ & $\mathrm{Cr}^{0}$ & & \\
\hline $\mathrm{V}$ & $3 \mathrm{~d}^{3} 4 \mathrm{~s}^{2}$ & $\mathrm{~V}^{5+}$ & $\mathrm{V}^{4+}$ & $\mathrm{V}^{3+}$ & $\mathrm{V}^{2+}$ & $\mathrm{V}^{+}$ & $\mathrm{V}^{0}$ & & & \\
\hline $\mathrm{Ti}$ & $3 \mathrm{~d}^{2} 4 \mathrm{~s}^{2}$ & $\mathrm{Ti}^{4+}$ & $\mathrm{Ti}^{3+}$ & $\mathrm{Ti}^{2+}$ & $\mathrm{Ti}^{+}$ & $\mathrm{Ti}^{0}$ & & & & \\
\hline Sc & $3 \mathrm{~d}^{1} 4 \mathrm{~s}^{2}$ & $\mathrm{Sc}^{3+}$ & $\mathrm{Sc}^{2+}$ & $\mathrm{Sc}^{+}$ & $\mathrm{Sc}^{0}$ & & & & & \\
\hline \multicolumn{2}{|c|}{$\begin{array}{l}\text { Electronic } \\
\text { configura- } \\
\text { tion } \mathrm{d}^{n}\end{array}$} & $3 \mathrm{~d}^{0}$ & $3 \mathrm{~d}^{1}$ & $3 \mathrm{~d}^{2}$ & $3 \mathrm{~d}^{3}$ & $3 d^{4}$ & $3 \mathrm{~d}^{5}$ & $3 \mathrm{~d}^{6}$ & $3 \mathrm{~d}^{7}$ & $3 \mathrm{~d}^{8}$ \\
\hline \multicolumn{2}{|c|}{$\begin{array}{l}\text { Number of } \\
\mathrm{d}^{n} \text { electrons }\end{array}$} & 0 & 1 & 2 & 3 & 4 & 5 & $6(4)$ & $7(3)$ & $8(2)$ \\
\hline \multicolumn{2}{|c|}{$\begin{array}{l}\text { Spin } \\
S=n / 2\end{array}$} & 0 & $1 / 2$ & 1 & $3 / 2$ & 2 & $5 / 2$ & 2 & $3 / 2$ & 1 \\
\hline \multicolumn{2}{|c|}{ Term ${ }^{2 S+1} L$} & ${ }^{1} S$ & ${ }^{2} D$ & ${ }^{3} \mathrm{~F}$ & ${ }^{4} F$ & ${ }^{5} D$ & ${ }^{6} S$ & ${ }^{5} D$ & ${ }^{4} F$ & ${ }^{3} F$ \\
\hline
\end{tabular}


$\mathrm{d}^{5}$ electronic configuration. Additional, table 2.1 presents the spin $S$ of the $3 \mathrm{~d}$-shell and the ground state term ${ }^{2 S+1} L$ of $3 \mathrm{~d}$-shell as function of the TM ionization, both values arise from Hund's rule [86; 87].

The incorporation of TM centers in wide band gap semiconductors results in a multiple splitting of degenerated 3d-shell states of free TM ions. The electronic structure is affected by the Stark effect of the crystal field, which has trigonal symmetry $\left(\mathrm{C}_{3 V}\right)$ in hexagonal wurtzite structures like $\mathrm{ZnO}$ and is treated as perturbation of a tetrahedral $\left(\mathrm{T}_{d}\right)$ symmetry of a cubic crystal $[21 ; 22]$. Further splitting of the electronic structure results from spin-orbit interaction, vibrational contributions and Jahn-Teller coupling [23]. As a result, transitions between these 3d-shell state become partly allowed and show very sharp transitions with long lifetimes (up to milliseconds) [22-25; 88]. In addition to spintronic applications, these TM intra-shell transitions within semiconductors could be used in optoelectronic devices.

Depending on the charge state of the transition metals donor or acceptor levels could be introduced into the band gap. Table 2.2 summarizes the expected charge state and its impact onto II-VI and III-V semiconductors [89]. Therefore, transition metals could be a hole or electron source, which is the case for $\mathrm{Mn}^{2+}$ ions in GaAs. For $\mathrm{ZnO}$, such transfer level was reported for copper impurities, which is a neutral acceptor [90].

This chapter gives an introduction to magnetic exchange interactions and summarizes the computed observations with emphasis onto transition metal alloyed ZnO. The discussion is continued with a short introduction to the degeneration of open inner shells $\left(d^{n}\right.$ and $f^{n}$ ) by the electrostatic crystal field of the host lattice in section 2.2. Finally, the anticipated impact onto the band gap of $\mathrm{ZnO}$ with increasing transition metal content is treated in section 2.3 .

Table 2.2: Expected oxidation and charge state of some transition metals in II$V I$ and III-V semiconductors, whereas all $T M^{2+}$ ions in $Z n O$ are typical neutral (denoted as $A^{0} / D^{0}$ ) [89].

\begin{tabular}{llllll}
\hline II-VI & $3 \mathrm{~d}^{3}$ & $3 \mathrm{~d}^{4}$ & $3 \mathrm{~d}^{5}$ & $3 \mathrm{~d}^{6}$ & $\mathrm{III}-\mathrm{V}$ \\
\hline $\mathrm{A}^{-}$ & & & $\mathrm{Cr}^{+}$ & $\mathrm{Mn}^{+}$ & $\mathrm{A}^{--}$ \\
$\mathrm{A}^{0} / \mathrm{D}^{0}$ & & $\mathrm{Cr}^{2+}$ & $\mathrm{Mn}^{2+}$ & $\mathrm{Fe}^{2+}$ & $\mathrm{A}^{-}$ \\
$\mathrm{D}^{+}$ & $\mathrm{Cr}^{3+}$ & $\mathrm{Mn}^{3+}$ & $\mathrm{Fe}^{3+}$ & & $\mathrm{A}^{0} / \mathrm{D}^{0}$ \\
$\mathrm{D}^{++}$ & $\mathrm{Mn}^{4+}$ & $\mathrm{Fe}^{4+}$ & & & $\mathrm{D}^{+}$ \\
\hline
\end{tabular}




\subsection{Magnetism}

\subsubsection{Direct and super-exchange}

The coupling of localized electron spins $\mathbf{s}_{i, j}$ can be described by the direct exchange interaction via the Heisenberg Hamiltonian [91-93]:

$$
H=\sum_{i j} J_{i j} \mathbf{s}_{i} \cdot \mathbf{s}_{j}
$$

where $J_{i j}$ is the exchange integral. $J_{i j}$ tends to be positive, if both states coupled by the exchange integral $J_{i j}$ are electronic states in a free atom. A positive exchange integral $J_{i j}$ represents a parallel alignment of spins. The situation changes completely for the spin exchange interaction between neighboring atoms. In that case $J_{i j}$ tends to be negative where two electrons are aligned antiparallel to form bonding states [93]. The sign of $J_{i j}$ in solids is in principle either positive or negative. This Heisenberg Hamiltonian (also 'Heisenberg Model') is used for the description of the ferromagnetism of transition metals, such as iron [91].

If the distance between the magnetic ions is too large for direct exchange coupling, the magnetic moments are coupled by the super-exchange mechanism [92; 93]. In this case the exchange interaction is mediated by means of a non-magnetic ion between the non-neighboring magnetic ions, occurring in ionic solids such as $\mathrm{MnO}$ [92]. The d-shell of $\mathrm{Mn}^{2+}$ ions has 5 electrons with parallel aligned spin, whereas the p-orbital of the $\mathrm{O}^{2-}$ ions is fully occupied with antiparallel spin alignment. The super-exchange mechanism mediates a parallel (ferromagnetic arrangement) or antiparallel (antiferromagnetic arrangement) alignment between two Mn ions via an oxygen ion. Thereby, the antiferromagnetic arrangement is energetically favored which allows a delocalization of the involved electrons due to a lowering of the kinetic energy [92]. It is important that the electrons of the oxygen atom are located within the same orbital, i.e. the oxygen atom must connect the both Mn atoms.

\subsubsection{Carrier-mediated exchange}

In carrier-mediated exchange processes the magnetic moments of transition metals are coupled via free carriers, requiring a high free carrier concentration. The RKKY ${ }^{1}$ exchange interaction occurs in metals with localized moments and the exchange is mediated via valence electrons (free electron gas) $[92 ; 93]$. A distance dependent

\footnotetext{
${ }^{1}$ RKKY: Ruderman, Kittel, Kasuya, Yosida
} 


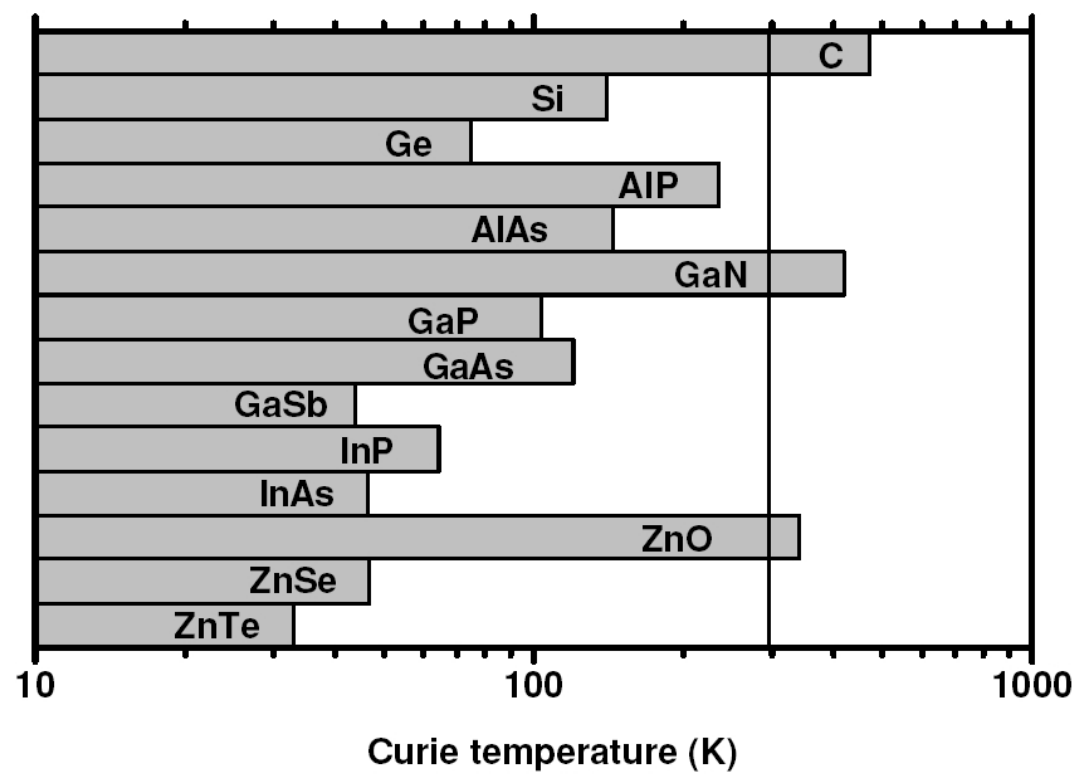

Figure 2.1: Computed Curie temperature for various semiconductors alloyed with 5 at.\% Mn with the assumption they show p-type conducten with a hole concentration of $3.5 \cdot 10^{20}$ per $\mathrm{cm}^{3}$ (from [3; 94]).

exchange integral characterizes the coupling between the magnetic moments [92]:

$$
J_{R K K Y}(r) \propto F\left(2 k_{F} r\right) \quad \text { with } \quad F(x)=\frac{\sin x-x \cos x}{x^{4}}
$$

This exchange coupling is of long range and anisotropic. Additionally, it possesses an oscillating behavior [92], resulting in a complicate spin arrangement. The ferroor antiferromagnetic ordering is a function of the distance between the magnetic moments. Rare earth metals are typically coupled via the RKKY exchange [92].

Dietl et al. proposes the mean-field Zener model to describe the ferromagnetic ordering in GaMnAs and ZnMnTe [3]. This model is based on the RKKY exchange interaction and the Zener model, whereas latter one proposes a spin exchange model between carriers and localized spins $[6 ; 95 ; 96]$. The d-shell spins of adjacent Mn atoms are aligned in an antiferromagnetic configuration due to super exchange interaction. On the other hand, the indirect coupling leads to a ferromagnetic alignment of the incomplete d-shell. Ferromagnetism could be observed, if the Zener coupling dominates over the direct super exchange coupling [3; 95; 96]. Dietl et al. assumes that the interaction between the localized Mn atoms is mediated by free holes in GaMnAs, and results in ferromagnetic ordering with a computed Curie tempera- 
ture of $120 \mathrm{~K}$. This computed value fits very well with the experimental value of $110 \mathrm{~K}$ for GaMnAs [80]. As Mn provides both a localized spin and at the same time an acceptor level within the band gap [89], Dietl et al. predicts the increase of the Curie temperature with increasing Mn content in GaMnAs [3], which was confirmed by experiments $[81 ; 82]$. On that basis Dietl et al. computed the Curie temperature for several p-type doped semiconductors containing 5 at. $\% \mathrm{Mn}$ with a hole concentration of $3.5 \cdot 10^{20}$ per $\mathrm{cm}^{3}$ (see figure 2.1) [3; 94]. Their results reveal a Curie temperature above room temperature for p-type $\mathrm{ZnO}, \mathrm{GaN}$ and diamond. As it is possible to prepare 5 at.\% $\mathrm{Mn}$ alloyed $\mathrm{ZnO}$ (see chapter 3), it is temporary impossible to prepare such high p-type doped $\mathrm{ZnO}$ crystals $[63 ; 97 ; 98]$.

\subsubsection{Double exchange}

Perovskite structure manganites $\mathrm{La}_{1-x} \mathrm{~A}_{x} \mathrm{MnO}_{3}$ (with $\mathrm{A}=\mathrm{Ca}$, Sr or $\mathrm{Ba}$ ) and transition metal oxides of the type $\mathrm{TM}_{3} \mathrm{O}_{4}$ include transition metal ions with two different charge states $[7 ; 92 ; 93]$. In these materials ferromagnetism could only be observed, if electronic conduction of this materials is good [7]. The oxygen ion is bound to two different charged transition metal ions. An electron can be transferred from the lower positive charged TM ion to the oxygen ion, if an electron is transfered from the oxygen ion to the higher positive charged TM ion at the same time [7]. Zener expresses two wave functions which represent the system configuration before $\psi_{1}$ and after $\psi_{2}$ the electron transfer for such manganese perovskite structure as [7]:

$$
\psi_{1}: \quad \mathrm{Mn}^{3+} \mathrm{O}^{2-} \mathrm{Mn}^{4+} \quad \psi_{2}: \quad \mathrm{Mn}^{4+} \mathrm{O}^{2-} \mathrm{Mn}^{3+}
$$

with an exchange energy of [7]:

$$
E_{\text {double }}=\int \psi_{1}^{*}\left(H-\epsilon_{0}\right) \psi_{2} d \tau
$$

where $H$ is the Hamiltonian of the whole system and $\epsilon_{0}$ the energy associated with the initial states $\psi_{1}$ and $\psi_{2}$. The integral is extended over the coordinates and spins of all electrons [7]. In compliance to Hund's rule, the hopping of electrons can only occur if the spins of both transition metal ions are aligned parallel $[7 ; 92]$.

Sato and Katayama-Yoshida have performed first principle electronic structure calculations for various II-VI and III-V semiconductors alloyed with transition metals $[4 ; 99-102]$. Their computed results for $\mathrm{ZnO}$ alloyed with $\mathrm{Mn}, \mathrm{Co}, \mathrm{Fe}$, or Ni are shown in figure 2.2 as function of carrier concentration for different transition metal concentrations. Thereby, the ferromagnetic state as well as the spin-glass state are 

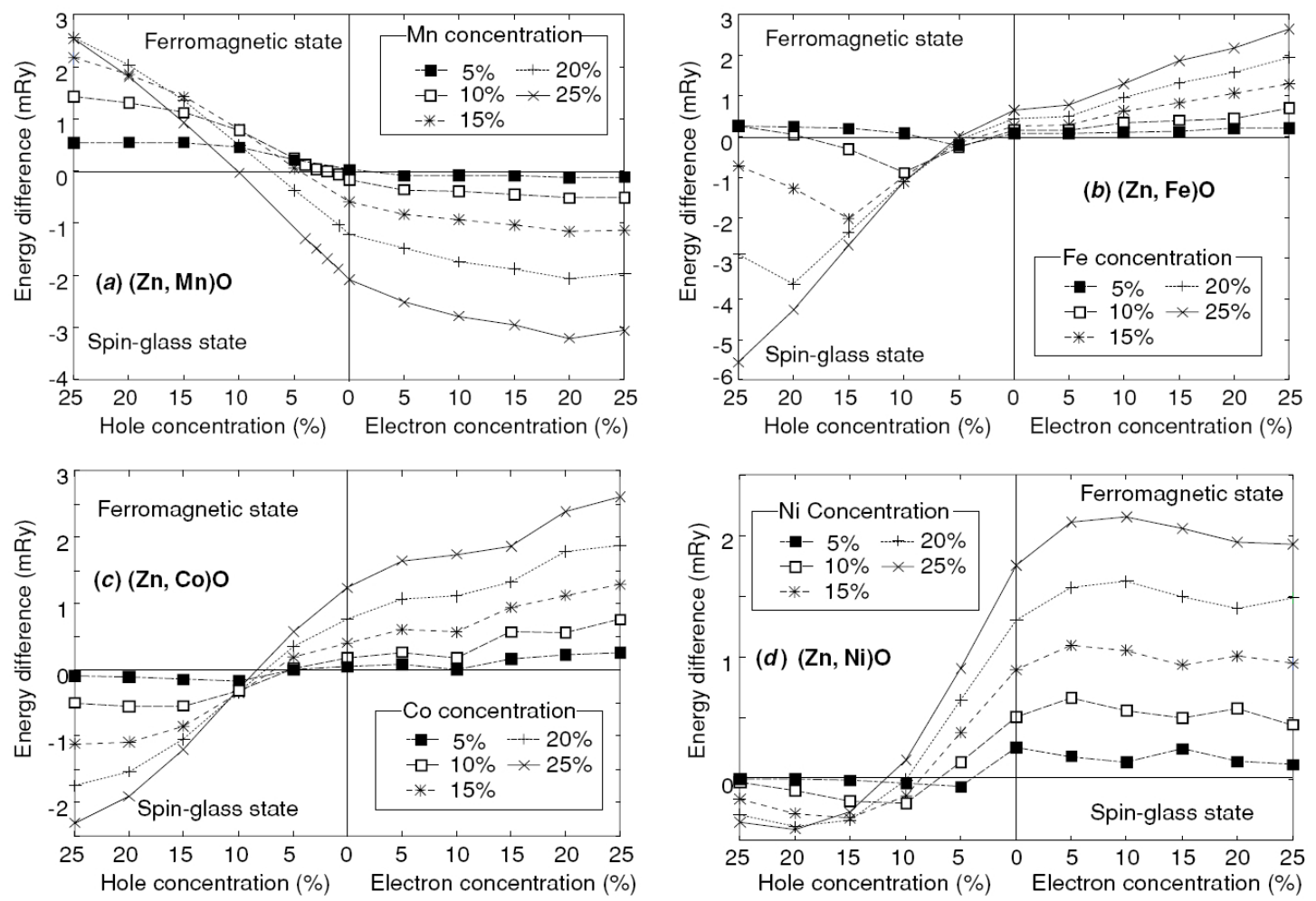

Figure 2.2: The stability of the ferromagnetic state in (a) Mn, (b) Fe, (c) Co and (d) Ni alloyed $\mathrm{ZnO}$ are shown as a function of the carrier concentration, which were computed by Sato and Katayama-Yoshida [99]. On the y-axis the energy difference between the ferromagnetic state and the spin-glass state is given.

indicated within the four diagrams. The y-axis demonstrates the stability of either the ferromagnetic state or the spin-glass state by means of the total energy difference between these two states and naively corresponds to the Curie temperature [99]. Their computed results exhibit the formation of a ferromagnetic state for TM alloyed $\mathrm{ZnO}$ in the series from $\mathrm{V}$ to $\mathrm{Ni}$ with exception of $\mathrm{Mn}$. For $\mathrm{Mn}$ alloyed $\mathrm{ZnO}$ they suggest that the super-exchange interaction between the Mn ion stabilizes the spin-glass state [99], due to the $\mathrm{d}^{5}$ electronic configuration. For transition metals with partially filled $3 \mathrm{~d}$ orbitals (3d-band of up-spin or down-spin states are not fully occupied) hopping of electrons from one TM ion to another TM ion by means of the double exchange interaction is allowed [99]. Figure 2.2 shows an increase in the energy difference of the ferromagnetic state with increasing electron concentration for $\mathrm{Fe}$, $\mathrm{Co}$ and $\mathrm{Ni}$ alloyed $\mathrm{ZnO}$, which results in a more stable ferromagnetic state and possibly in a higher Curie temperature [99]. In new publications, Sato and Katayama-Yoshida have simulated the Curie temperature of several diluted mag- 


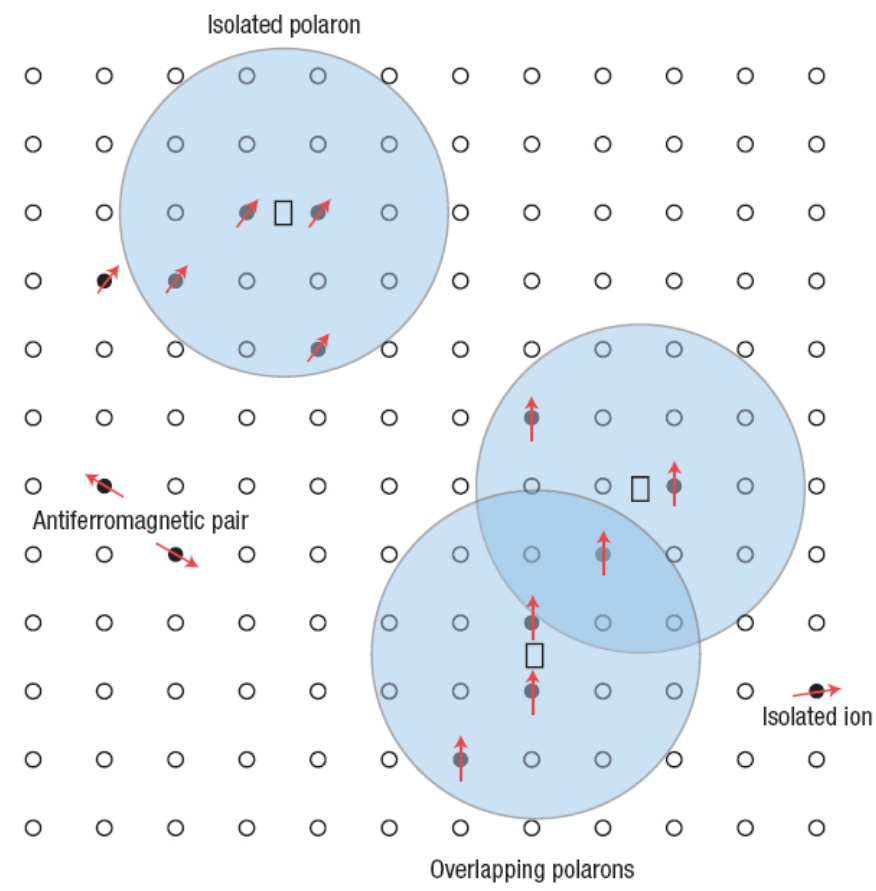

Figure 2.3: The spin of a bound magnetic polaron (donor electron) couples antiparallel to a half-full or more than half-full $3 d$-shell of a transition metal impurity (from [9]).

netic semiconductors via mean field approximation, random phase approximation or Monte Carlo simulation [103-105]. The mean field approximation suggests a Curie temperature of about $570 \mathrm{~K}$ for the calculated energy difference of $1.36 \mathrm{mRy}$ of $25 \%$ $\mathrm{V}$ alloyed $\mathrm{ZnO}[102]$.

\subsubsection{Bound magnetic polaron}

Given that $\mathrm{GaN}$ and $\mathrm{ZnO}$ are intrinsically n-type conducting, a high amount of shallow and deep donor levels within the band-gap could be found $[73 ; 106 ; 107]$ and act as electron traps. The formation of one charge-compensation electron for every donor defect leads to the production of a polaron [108-111], whereas the polaron size (hydrogenic orbital) depends on the effective mass of the donor electron and the dielectric constant [8]. In that case the donor tends to form a bound magnetic polaron, where the spin of the donor electron couples with 3d-moments of the transition metal ions inside the polaron orbit [108-111] and leads to the alignment of all 3d-moments [9]. This situation is shown in figure 2.3 [9], where the donor defects associated with the hydrogenic polaron orbits are represented as squares and blue 
circles, respectively. As shown in figure 2.3, all 3d-moments within an hydrogenic polaron orbit are aligned parallel. If two or more hydrogenic polaron orbits overlap, the $3 \mathrm{~d}$-moments within all the overlapping polaron orbits are aligned in the same direction. The interaction between the 3d-moments and the donor electron spin is represented by the Heisenberg exchange Hamiltonian (equation 2.1 in section 2.1.1) [8]. Therefore, it is possible to produce ferromagnetic diluted magnetic semiconductors where the ferromagnetism is mediated via defects and does not necessarily involve free charge carriers [8]. The Curie temperature depends on the donor and magnetic impurity concentration, as well as on the position of the impurity in the $3 \mathrm{~d}$-series [9]. The magnetic moment per dopant cation at room temperature of thin $\mathrm{Zn}_{0.95} \mathrm{TM}_{0.05} \mathrm{O}$ films shows a maximum at $\mathrm{Co}$, and between Ti and V [9].

\section{$2.23 d$-shell degeneration}

The incorporation of transition metal elements into a crystal lattice leads to an interaction between the host crystal and the $3 \mathrm{~d}$-shell of the transition metals. Bethe introduced in the early 20th century the crystal field theory, which describes the influence of an electric field with a predetermined symmetry onto an atom [112]. It should be noted, that this crystal field theory affects not only the partly filled $3 d$-shell of transition metals, it describes the splitting of $f^{n}$-shells as well. Thus, the following description of crystal field splitting is applied to all magnetic ions including the complete transition metal series (with partially filled $3 d-$, $4 d-$, or $5 d-$ shells), the lanthanide series (with partially filled $4 f$-shells) and the actinide series (with partially filled $5 f$-shells). The relative strength of the crystal field and other interactions differs between those magnetic ions, but the model of state splitting is essentially the same for all magnetic ions [113]. Otherwise, it was shown, that the crystal field is a significant factor for the susceptibility of paramagnetic iron group and rare earth group salts [114-116]. Therefore, the configuration of $d^{n}$ - and $f^{n}$ shell within semiconductors is a critical criterion for the above mentioned magnetic coupling mechanism. The electronic structure of a free atom can be solved via the Schrödinger equation [117]:

$$
\left(\sum_{i=1}^{Z}\left[\frac{\mathbf{p}_{i}^{2}}{2 m}-\frac{Z e^{2}}{r_{i}}+\sum_{i>j} \frac{e^{2}}{\left|\mathbf{r}_{i}-\mathbf{r}_{j}\right|}\right]\right) \psi(\mathbf{r})=E \psi(\mathbf{r})
$$

where the part in brackets is the Hamiltonian for an atom with the atomic number $Z$. For the crystal field perturbation the Hamiltonian for a free atom in equation 2.2 
is expanded with the crystal field interaction $H_{C F}$, spin-orbit interaction $H_{S O}$, and possible further interactions $[85 ; 113 ; 118]$ :

$$
H=\left(\sum_{i=1}^{N}\left[\frac{\mathbf{p}_{i}^{2}}{2 m}-\frac{Z e^{2}}{r_{i}}\right]+\sum_{i>j} \frac{e^{2}}{\left|\mathbf{r}_{i}-\mathbf{r}_{j}\right|}\right)+H_{C F}+H_{S O}+\ldots ;
$$

where the first term represents the Hamiltonian for a free transition metal ion and $N$ the number of electrons. Bethe used the group theory for the development of the crystal field theory [112]. Due to the complexity and length of the whole theory, a full description of the theory would go beyond the scope of this thesis. Therefore, only a short introduction into the crystal field theory and the main perturbation is presented within this section, a more detailed account is given in the references $[85 ; 112 ; 113 ; 118-122]$ and references therein.

\subsubsection{Crystal field perturbation}

The crystal field theory describes the effect of electrostatic forces of a host material onto the electronic configuration of a magnetic ion $[113 ; 119]$. The nature of bonding is one factor. In ionic crystals the electrostatic force is divided into a long-range attractive force and a short-range repulsive force, which is substantially assigned to the outer electrons acting between neighboring ions [113]. Covalent bonded materials share the electrons between orbitals of neighboring ions, which reduces the total energy of the system [113]. Within a crystal the electric forces onto an magnetic ion degrade the free ion electron states by the same mechanism which produces crystal bonding [113]. For this degeneration an additional Hamiltonian $H_{C F}$ is introduced into equation 2.2 and is specified as $[113 ; 119]$ :

$$
H_{C F}=-e V_{C F} \text {. }
$$

The intensity of the crystal field splitting is then determined by evaluation of the matrix elements [119]:

$$
\int \psi_{i}^{*} \cdot e V_{C F} \cdot \psi_{j} d \tau \equiv\left\langle\psi_{i}\left|e V_{C F}\right| \psi_{j}\right\rangle
$$

where $\psi_{i, j}$ are spherical wave functions. The main question at this point is: how to proceed with the $\psi$ wave functions on $V_{C F}$, which requires a detailed knowledge of the potential operator $V_{C F}$. Hence, a mathematical form of $V_{C F}$ is established via the expansion theorem, which is in detail described in reference [119]. In this way, 
the crystal-field potential $V_{C F}$ in equation 2.4 can be written as $[113 ; 119]$ :

$$
V_{C F}=\sum_{k} \sum_{q} c_{k}^{q} R_{k}(r) Y_{k}^{q}(\theta, \phi)
$$

where $c_{k}^{q}$ are expansion coefficients of $V_{C F}$ with respect to the basis functions $R_{k}(r) Y_{k}^{q}(\theta, \phi)$. Thereby, $R_{k}(r)$ is a radial function and $Y_{k}^{q}(\theta, \phi)$ a spherical harmonic. Equation 2.5 expresses the potential $V_{C F}$ as sum of potentials from all the surrounding ligands [119]. The crystal field potential energy of a magnetic ion can then be expressed as sum of individual electronic contributions of the crystalline environment and is solved in terms of many-electron matrix elements (more details in $[113 ; 119 ; 121])$, which includes one electron in an open-shell orbital of the magnetic ion and all outer $\mathrm{s}^{2} \mathrm{p}^{6}$-shell electrons of the ligands. The terms $R_{k}(r) Y_{k}^{q}(\theta, \phi)$ in equation 2.5 are called 'crystal field parameters'. For an electron with an angular momentum $l$, a linear expansion of the operator $W_{C F}$ is introduced to remove the dependence on angular coordinates, which generalizes the multipolar expansion of equation 2.5 [113]:

$$
\left\langle l m_{1}\left|W_{C F}\right| l m_{2}\right\rangle=\sum_{k, q}\left\langle l m_{1}\left|t_{q}^{(k)}\right| l m_{2}\right\rangle \hat{B}_{q}^{k}
$$

Thereby, the $\hat{B}_{q}^{k}$ are the crystal field parameters, which are in general complex quantities. The $m_{i}$ are the magnetic quantum numbers with values between $-l$ and $+l$. $\left\langle l m_{1}\left|t_{q}^{(k)}\right| l m_{2}\right\rangle$ are the matrix elements of the one-electron matrix operators $t_{q}^{(k)}$ (defined in Appendix 1 of reference [113]). In this vein, the crystal field potential in equation 2.5 can be supposed as crystal field operator acting on many-electron states and is written as [113]:

$$
V_{C F}=\sum_{k, q} \hat{B}_{q}^{k} T_{q}^{(k)} \quad \text { with } \quad T_{q}^{(k)}=\sum_{i} t_{q}^{(k)}(i)
$$

As illustrated in equation 2.7, the tensor operator $T_{q}^{(k)}$ is the sum of the one-electron tensor operators $t_{q}^{(k)}(i)$ from equation 2.6, which acts on the states of single electrons [113]. In quantum mechanics an energy operator must be Hermitian; thus, the crystal field operator in formula 2.7 must be Hermitian. Therefore, the Hermitian conjugates of the tensor operator $T_{q}^{(k)}$ and the crystal field parameter are given by [113]:

$$
T_{q}^{(k) \dagger}=(-1)^{q} T_{-q}^{(k)} \quad \text { and } \quad \hat{B}_{q}^{k \dagger}=(-1)^{q} \hat{B}_{-q}^{k}
$$

Consequently the crystal field operator $V_{C F}$ from equation 2.7 can be written as 
[113]:

$$
V_{C F}=\sum_{k, q} B_{q}^{k} \Omega_{k, q}
$$

where $B_{q}^{k}$ is defined for $q=0: \hat{B}_{0}^{k}=B_{0}^{k}$; and for $q>0$ :

$$
\hat{B}_{q}^{k}=B_{q}^{k}+\mathrm{i} B_{-q}^{k} \quad \text { and } \quad \hat{B}_{q}^{k \dagger}=(-1)^{q}\left(B_{q}^{k}-\mathrm{i} B_{-q}^{k}\right)
$$

Therefore, the crystal field is now expressed in terms of the real parameter $B_{q}^{k}$ and the tensor operator $T_{q}^{(k)}$ in equation 2.7 is replaced by the operator $\Omega_{k, q}$, which forms no tensor [113]. The latter is expressed by [113]:

$$
\begin{aligned}
& \Omega_{k, q}=\left(T_{q}^{(k)}+(-1)^{q} T_{-q}^{(k)}\right), \text { for } q>0 \\
& \Omega_{k, q}=\mathrm{i}\left(T_{-q}^{(k)}+(-1)^{q} T_{q}^{(k)}\right), \text { for } q<0 \\
& \Omega_{k, 0}=T_{0}^{(k)} .
\end{aligned}
$$

It is now necessary to assign numerical values to the crystal field parameter $B_{p}^{k}$.

Table 2.3: Listed are the algebraic expressions of the tensor operators $C_{q}^{(k)}$ with $q \geq 0$ (after [113]). For negative $q: r_{+}=x+i y$ is replaced with $r_{-}=x-i y$ and the overall expression is multiplied by the factor $(-1)^{q}$.

\begin{tabular}{lll}
\hline$k$ & $q$ & $r^{k} C_{q}^{(k)}$ \\
\hline 2 & 0 & $\frac{1}{2}\left(3 z^{2}-r^{2}\right)$ \\
2 & 1 & $-\frac{1}{2} \sqrt{6} z r_{+}$ \\
2 & 2 & $\frac{1}{4} \sqrt{6} r_{+}^{2}$ \\
4 & 0 & $\frac{1}{8}\left(35 z^{4}-30 r^{2} z^{2}+3 r^{4}\right)$ \\
4 & 1 & $-\frac{1}{4} \sqrt{5}\left(7 z^{2}-3 r^{2}\right) z r_{+}$ \\
4 & 2 & $\frac{1}{8} \sqrt{10}\left(7 z^{2}-r^{2}\right) r_{+}^{2}$ \\
4 & 3 & $-\frac{1}{4} \sqrt{35} z r_{+}^{3}$ \\
4 & 4 & $\frac{1}{16} \sqrt{70} r_{+}^{4}$ \\
6 & 0 & $\frac{1}{16}\left(231 z^{6}-315 r^{2} z^{4}+105 r^{4} z^{2}-5 r^{6}\right)$ \\
6 & 1 & $-\frac{1}{16} \sqrt{42}\left(33 z^{4}-30 r^{2} z^{2}+5 r^{4}\right) z r_{+}$ \\
6 & 2 & $\frac{1}{32} \sqrt{105}\left(33 z^{4}-18 r^{2} z^{2}+r^{4}\right) r_{+}^{2}$ \\
6 & 3 & $-\frac{1}{16} \sqrt{105}\left(11 z^{2}-3 r^{2}\right) z r_{+}^{3}$ \\
6 & 4 & $\frac{3}{32} \sqrt{14}\left(11 z^{2}-r^{2}\right) r_{+}^{4}$ \\
6 & 5 & $-\frac{3}{16} \sqrt{77} z r_{+}^{5}$ \\
6 & 6 & $\frac{1}{32} \sqrt{231} r_{+}^{6}$ \\
\hline
\end{tabular}




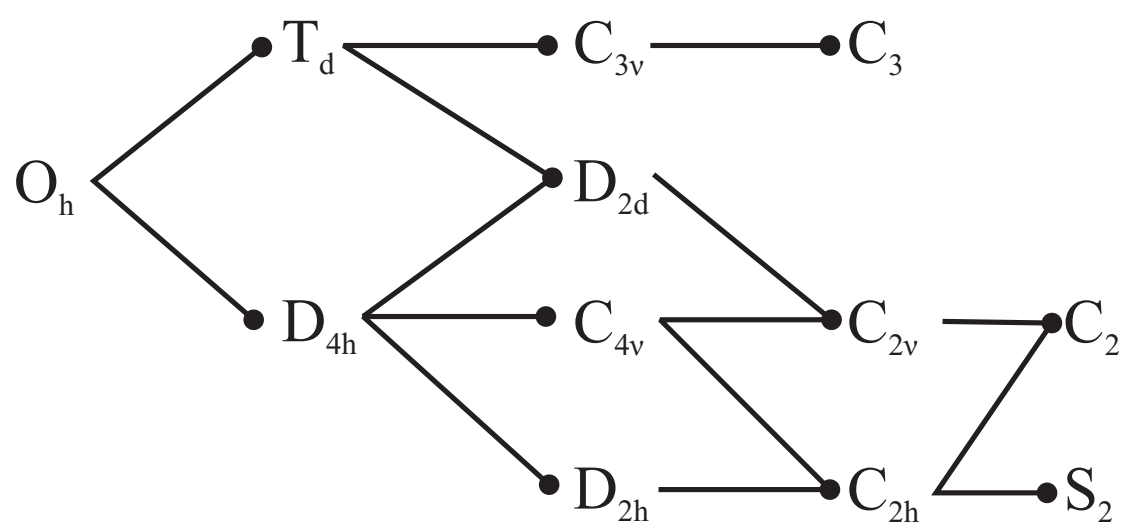

Figure 2.4: Crystal field $O_{h}$ symmetries and their subgroups (after [113]).

For this it is needed to adopt a specific normalization of the tensor operator $t_{q}^{(k)}(i)$. This is done via the so-called 'Wybourne normalization'by use of tensor operators which have the same normalization as the functions $C_{q}^{(k)}(i)=C_{q}^{(k)}\left(\theta_{i}, \phi_{i}\right)$ [113]. These functions are related to spherical harmonics [113]:

$$
C_{q}^{(k)}(i)=\sqrt{\frac{4 \pi}{2 k+1}} Y_{k, q}\left(\theta_{i}, \phi_{i}\right)
$$

And with the Hermitian combination of the complex functions $C_{q}^{(k)}(i)$, as in equation 2.9 , the crystal field operator is then written as [113]:

$$
V_{C F}=\sum_{k, q>0}\left[B_{q}^{k}\left(C_{q}^{(k)}+(-1)^{q} C_{-q}^{(k)}\right)+B_{-q}^{k} \mathrm{i}\left(C_{-q}^{(k)}-(-1)^{q} C_{q}^{(k)}\right)\right]+\sum_{k} B_{0}^{k} C_{0}^{(k)},
$$

where $C_{q}^{(k)}=\sum_{i} C_{q}^{(k)}(i)$ is the summation over all the electrons in the $\mathrm{d}^{n}$ - or $\mathrm{f}^{n}$-shell. Table 2.3 gives the list of the algebraic expression of the different tensor operators $C_{q}^{(k)}$ for different $k$ and $q$ combinations [113]. Site symmetry and matrix element selection rules are used to determine which tensor operator components in table 2.3 are introduced into the crystal field potential 2.11, whereas the restrictions are understood by the group theory [113]. For most magnetic ions the site symmetry group within a crystal lattice is a subgroup of the full rotation group $\mathrm{O}_{3}$ and are shown in figure 2.4 (drawn according to reference [113]). Transition metal ions in ZnO have a $\mathrm{C}_{3 \nu}$ site symmetry [22], which is a subgroup of the tetrahedral $\mathrm{T}_{d}$ side symmetry and therefore a subgroup of the $\mathrm{O}_{3}$ group (see figure 2.4). For a given $k$ the set of crystal field parameters of a $\mathrm{O}_{3}$ subgroup is determined by the number of site symmetry invariants for each of the allowed $k$ values [113], whereas a subgroup 
Table 2.4: Non-vanishing crystal field parameters which are indicated by '+' for $+q$ and \pm if $+q$ and $-q$ parameters are non-zero (after [113]).

\begin{tabular}{ccccccccc}
\hline$k$ & $|q|$ & $\mathrm{C}_{3 \nu}$ & $\mathrm{C}_{3 h} / \mathrm{D}_{3 h}$ & $\mathrm{C}_{2}$ & $\mathrm{D}_{2 d} / \mathrm{C}_{2 \nu}$ & $\mathrm{D}_{2 h}$ & $\mathrm{~S}$ & $\mathrm{D}_{4 h}$ \\
\hline 2 & 0 & + & + & + & + & + & + & + \\
2 & 2 & & & \pm & + & + & \pm & \\
4 & 0 & + & + & + & + & + & + & + \\
4 & 2 & & & \pm & + & + & \pm & \\
4 & 3 & + & & & & & & \\
4 & 4 & & & \pm & + & + & \pm & + \\
6 & 0 & + & + & + & + & + & + & + \\
6 & 2 & & & \pm & + & + & \pm & \\
6 & 3 & + & & & & & & \\
6 & 4 & & & \pm & + & + & \pm & + \\
6 & 6 & + & + & \pm & + & + & \pm & \\
\hline
\end{tabular}

contains one or more invariant representations. Table 2.4 shows the selection rules for the crystal field potential components [113]. For $\mathrm{ZnO}$ it is common to handle the $\mathrm{C}_{3 \nu}$ crystal field perturbation as further perturbation of the tetrahedral $\mathrm{T}_{d}$ crystal field $[22 ; 23]$.

\subsubsection{Spin-orbit interaction}

Not only the crystal field results in a splitting of inner-shell states of magnetic ions, also the magnetic dipole-dipole interaction between the spin $s_{i}$ and the angular magnetic moments $l_{i}$ of inner-shell electrons of magnetic ions results in energy level splitting. The Hamiltonian for the spin-orbit interaction can be written as [113]:

$$
H_{S O}=\zeta \sum_{i} \mathbf{s}_{i} \cdot \mathbf{l}_{i}
$$

where $\mathbf{s}_{i}$ and $\mathbf{l}_{i}$ are the spin and angular momentum operators for the $d$ or $f$ electron labeled $i . \zeta$ in equation 2.12 is the spin orbit coupling constant.

For transition metal ions in crystals the spin-orbit interaction is small compared to the crystal field interaction, which is the opposite way around for rare earth ions where spin-orbit interaction is dominating [122]. Hence, the spin-orbit interaction is 
neglected in a first approximation, where the crystal field interaction is determined ${ }^{2}$. As second approximation the spin-orbit interaction is determined.

\subsubsection{Kramers degeneracy}

A non degenerated state within a free ion in free space is only possible for evennumbered electronic system [120], then we have $\mathbf{J}=0$ for such systems ${ }^{3}$. But all odd-numbered electron states show a degeneracy where the degree is increased by a magnetic field [120]. Kramer shows that all states of an odd-numbered electronic system must still have a degeneracy in an electrostatic field in the absence of a magnetic field $[120 ; 121 ; 123]$. The degeneracy is a consequence of the invariance of the electron Hamiltonian to time-reversal, which follows from the antiunitary $T$ operator to wave function of an odd-number of electrons [123]. The electronic energy level splits into at least two states [121], whereas a Kramer degenerated electron state are named Kramers doublet [120]. It should be additionally noted that Kramers theorem does not exclude the double degeneracy of electron levels in even-numbered electron systems [121].

\subsubsection{Jahn-Teller distortion}

The Jahn-Teller distortion (also known as 'Jahn-Teller effect') describes the distortion of a molecule or crystal lattice to achieve a lower energy and a lower degeneration in a system with static environment [120; 124]. Therefore, the Jahn-Teller distortion removes the degeneration of an degenerated ground state [124]. Figure 2.5 shows an example considered by Jahn and Teller [120]. On the vertices of a square four identical closed-shell ions are arranged together with an ion in the center of the square. Thereby, latter one has closed shells with a single $p$ electron outside. The position of this $p_{x^{-}}$or $p_{y}$-orbitals are indicated by two ellipses within the squares in figure $2.5 \mathrm{a}$ and figure $2.5 \mathrm{~b}$, respectively. These orbitals are necessarily degenerated [120]. The electrostatic potential of the environment affecting the p-orbitals is than written as [120]:

$$
V=\beta_{00}+\beta_{20} Z_{20}
$$

where $Z_{l m}$ is the real quantity of a spherical harmonic and $\beta_{k \alpha}$ is a suitable function to describe the environmental configuration. Due to the distortion of the environ-

\footnotetext{
${ }^{2}$ For $\mathrm{ZnO}$ the first approximation deals only with the crystal field interaction in $\mathrm{T}_{d}$ symmetry, the trigonal field is added as third approximation after the spin-orbit interaction [22].

${ }^{3} \mathbf{J}$ is the total angular momentum with: $\mathbf{J}=\mathbf{L}+\mathbf{S}$
} 


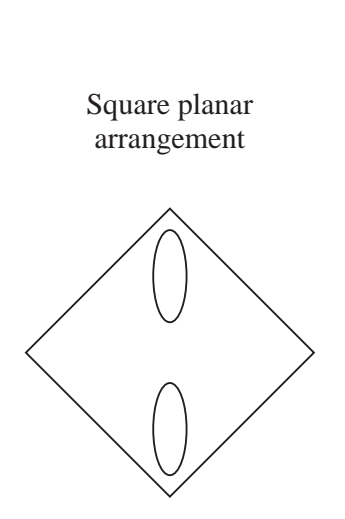

(a)

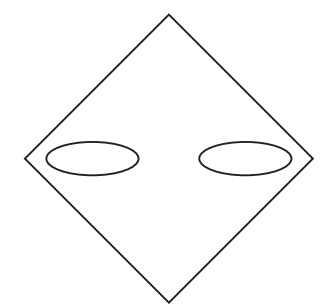

(d)

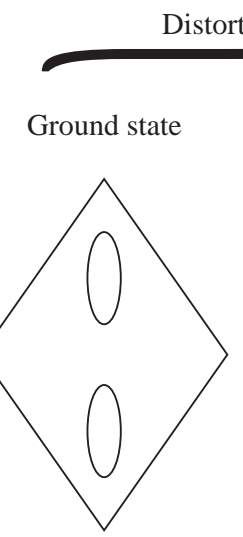

(b)

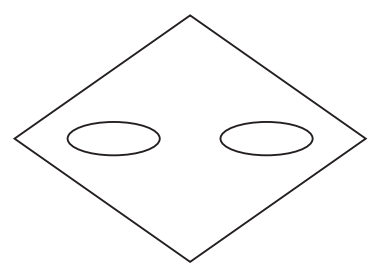

(e)

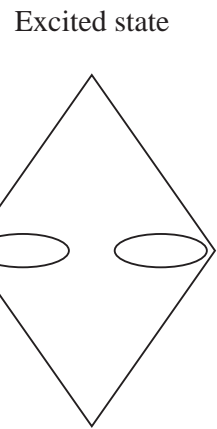

(c)

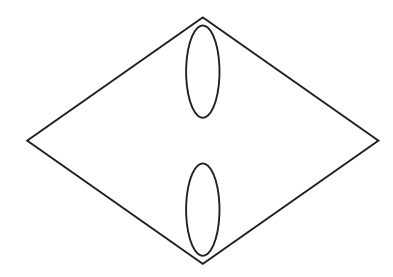

(f)

Figure 2.5: Schematic drawing of the Jahn-Teller effect in a planar compound

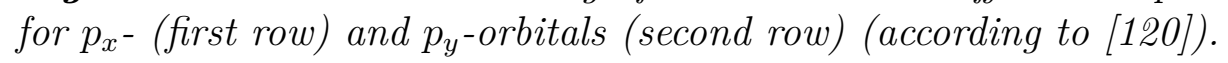

ment, by pushing two neighbors in and pulling two others out by the same amount [as visible in figure 2.5(b), (c), (e), and (f)], an additional term is inserted into the potential, whereby the parameter $\eta$ describes the degree of the distortion [120]:

$$
V(\eta)=\beta_{00}+\beta_{20} Z_{20}+\beta_{22}^{c}(\eta) Z_{22}^{c}
$$

where $\beta_{22}^{c}$ is zero, if $\eta$ is zero and if $\eta=\eta_{1}$ for the situation in figure 2.5(b) and (c), then $\eta=-\eta_{1}$ for the equal but opposite distortion shown in figure 2.5(e) and (f). The potentials for both configurations are coupled via the vector transformation matrix $C^{z}[120]$ :

$$
V(-\eta)=C^{z} V(\eta) \text { and therefore: } \quad \beta_{22}^{c}(-\eta)=-\beta_{22}^{c}(\eta)
$$

The energy of a distorted configuration is then given by [120]:

$$
E_{x x}=\left\langle p_{x}\left|\beta_{22}^{c} Z_{22}^{c}\right| p_{x}\right\rangle \quad \text { and } \quad E_{y y}=\left\langle p_{y}\left|\beta_{22}^{c} Z_{22}^{c}\right| p_{y}\right\rangle
$$


and depends on $\eta$, whereas both energies are coupled via the vector transformation matrix $C^{z}: C^{z} E_{x x}=-E_{y y}$.

For atoms in a macroscopic crystal the physics are slightly different, where the Jahn-Teller distortion is induced by the vibration of the nuclear framework [121]. The Hamiltonian of a system with $N$ electrons and $N_{0}$ nuclei can be written as [121]:

$$
\begin{aligned}
H_{J T} & =H_{E}+H_{N}+H_{N E}, \\
\text { with: } H_{E} & =-\frac{\hbar^{2}}{2 m} \sum_{i=1}^{N} \Delta_{e i}+\sum_{i>j=1}^{N} \frac{e^{2}}{r_{i j}}, \\
H_{N} & =-\sum_{k=1}^{N_{0}} \frac{\hbar^{2}}{2 M_{k}} \Delta_{n k}+\sum_{i>j=1}^{N} \frac{Z_{k} Z_{l} e^{2}}{R_{k l}}, \\
H_{N E} & =-\sum_{k=1}^{N_{0}} \sum_{i=1}^{N} \frac{Z_{k} e^{2}}{r_{i k}},
\end{aligned}
$$

where $H_{E}$ is the Hamiltonian for the electron energy, $H_{N}$ the nuclei energy and $H_{N E}$ the nuclei-electron interaction. Where $\Delta_{e i}$ is the Laplace operator of the $i$ th electron coordinates; $\Delta_{n k}$ is the Laplace operator of the $k$ th nuclear coordinates; $R_{k l}$ is the nuclear distance between nuclei $k$ and $l ; r_{i k}$ is the distance between electron $i$ and nucleus $k ; Z_{k} e$ is the charge of the nucleus $k$, and $M_{k}$ is the mass of nucleus $k$ [121]. The Hamiltonian is solved with an electronic wave function $\psi(\mathbf{r}, \mathbf{R})$, whereas the nuclei are fixed at $R$ [121]: ${ }^{4}$

$$
\left(H_{E}+\sum_{k>l} \frac{Z_{k} Z_{l} e^{2}}{R_{k l}}+H_{E N}\right) \psi(\mathbf{r}, \mathbf{R})=U_{\mu}(\mathbf{R}) \psi(\mathbf{r}, \mathbf{R})
$$

where $\mu$ is a set of quantum numbers. $\psi(\mathbf{r}, \mathbf{R})$ is considered as function of $\mathbf{r}$ with parameter $\mathbf{R}$, then the total wave function for the Hamiltonian in 2.14 can be written as [121]: $\Psi(\mathbf{r R})=\psi(\mathbf{r}, \mathbf{R}) \chi(\mathbf{R})$. Then, $\chi(\mathbf{R})$ is determined by [121]:

$$
\left[-\sum_{k=1}^{N_{0}} \frac{\hbar^{2}}{2 M_{k}} \Delta_{n k}+U_{\mu}(\mathbf{R})\right] \chi_{\mu \nu}(\mathbf{R})=E_{\mu \nu} \chi_{\mu \nu}(\mathbf{R}) .
$$

The separation of the equations of motion for the electron and nuclei from equation 2.14 to equation 2.15 is called Born-Oppenheimer approximation [121]. The potential $U(\mathbf{R})$ stimulates the nuclear motion and leads to a displacement of the nucleus around the equilibrium position $\mathbf{R}_{0 k}$ and its form depends on the electronic state $\mu$

\footnotetext{
${ }^{4}$ Due to the great difference in the velocity and mass between the electron and the nuclei, the nuclear kinetic energy term is neglected [121].
} 
[121]. This displacement induces a deformation of the electron orbital, but no jump from one orbital into another [121]. The Jahn-Teller distortion lowers the local symmetry, which lifts the degeneracy and these states are described as 'vibronic' [125]. The Jahn-Teller distortion is subdivided into a static Jahn-Teller distortion and a dynamic Jahn-Teller distortion. In static Jahn-Teller distortion the introduction of anharmonicity may force the system into a stable nuclear configuration [121], which is absence for the dynamic Jahn-Teller distortion. If the motions of electrons and nucleis are strongly coupled, then the systems moves from one equilibrium configuration to another, which is called dynamic Jahn-Teller distortion [121]. It was shown, that the Jahn-Teller distortion may not be excluded from the physics of intra-shell transitions of transition metal doped wide band gap semiconductors [21; 126-128].

\subsection{Band-gap}

So far only the theory behind the magnetism and the 3d-shell degeneration of transition metals in semiconductors are discussed with emphasis to ZnO. With increasing TM concentration replacing zinc atoms the physical properties are modified and the influence of the alloyed transition metal should rise. The band gap should be a part of the consideration for transition metal alloyed $\mathrm{ZnO}$ in the same way. The band gap of $\mathrm{ZnO}$ is increased by replacing $\mathrm{Zn}$ with $\mathrm{Mg}$ and decreased by replacing $\mathrm{Zn}$ with Cd $[98 ; 129]$. For these cases the band gap $E_{g}$ is a function of the concentration $x$ of $\mathrm{Mg}$ or $\mathrm{Cd}$ in the ternary semiconductor $\mathrm{A}_{x} \mathrm{Zn}_{1-x} \mathrm{O}$ (where $\mathrm{A}=\mathrm{Mg}$ or $\mathrm{Cd}$ ) [98]:

$$
E_{g}(x)=(1-x) E_{Z n O}+x E_{A O}-b x(1-x),
$$

where $b$ is the bowing parameter, $E_{A O}$ and $E_{Z n O}$ are the band-gap energies of the compounds $\mathrm{AO}$ and $\mathrm{ZnO}$. The bowing parameter $b$ is a function of the difference in electronegativity of the binaries $\mathrm{AO}$ and $\mathrm{ZnO}$ [98]. For $\mathrm{Mg}$ alloyed $\mathrm{ZnO}$ the band gap $E_{g}$ increases nearly up to $4.15 \mathrm{eV}$ for $\mathrm{Mg}_{0.36} \mathrm{Zn}_{0.64} \mathrm{O}$, for higher $\mathrm{Mg}$ concentrations the form of $\mathrm{MgO}$ segregations were observed [98]. A similar observation is anticipated for transition metal alloyed $\mathrm{ZnO}$, whereas the direction (increase or decrease) of the band gap depends first on the concentration of the selected transition metal and furthermore on the size of the transition metal monoxide band gap. The latter one is specified in table 2.5, where theoretically and/or experimentally determined values reveal a higher band gap for $\mathrm{MnO}$ and $\mathrm{NiO}$ than for $\mathrm{ZnO}$ [130; 131]. FeO, $\mathrm{CoO}$ and $\mathrm{CuO}$ have lower band gaps than $\mathrm{ZnO}$, and $\mathrm{TiO}$ and $\mathrm{VO}$ are metallic conductive compounds, and are therefore not included into tabel 2.5 [130-132]. 


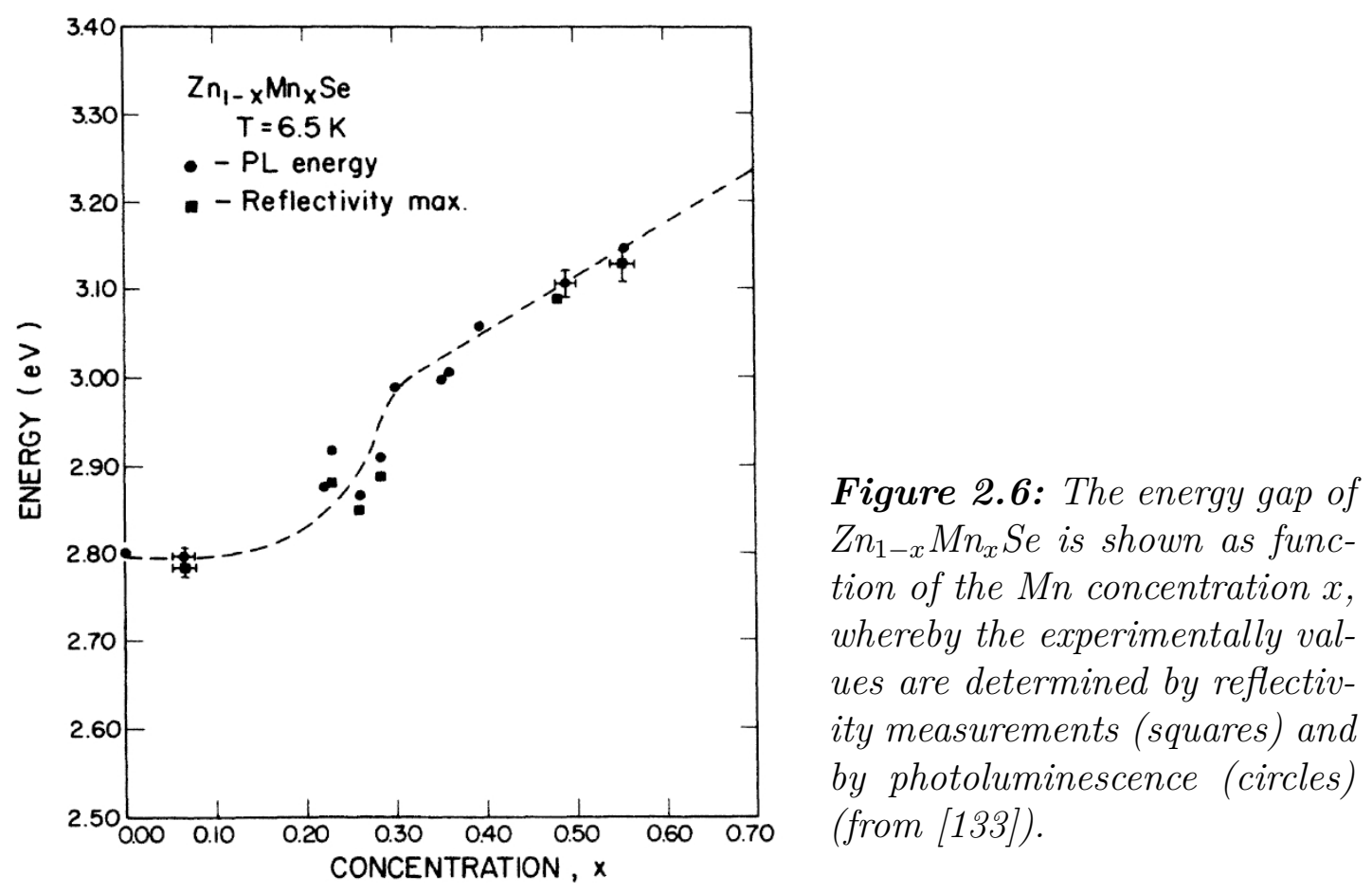

For $\mathrm{Zn}_{1-x} \mathrm{Mn}_{x}$ Se and $\mathrm{Cd}_{1-x} \mathrm{Mn}_{x} \mathrm{~S}$ alloys an increase of the band gap with increasing Mn concentration was found, which shows an anomalous drop of the band gap for low Mn concentrations [79; 133]. Figure 2.6 shows this anomalous drop of the band gap for $\mathrm{Zn}_{1-x} \mathrm{Mn}_{x}$ Se below $x \leq 0.3$ with an linear increase of the band gap above this value [133]. This behavior could not be explained by the differences in electronegativity as specified in equation 2.16 by the bowing parameter $b$. Bylsma et al. refers the anomalous band gap bowing to $s p-d$ exchange interaction [133], which is known to occur in diluted magnetic semiconductors. The $s p-d$ exchange interaction leads to a slight broadening of the d-levels of transition metals into flat and narrow bands [79].

Bylsma et al. shows a correlation between the $s p-d$ exchange interaction and the eigenenergies of the band carriers [133]. By second-order perturbation theory Bylsma et al. found a correlation between the paramagnetic susceptibilities of those

Table 2.5: Band gap $E_{g}$ of some 3d-metal monoxides [130-132].

\begin{tabular}{lllllll}
\hline & $\mathrm{ZnO}$ & $\mathrm{MnO}$ & $\mathrm{FeO}$ & $\mathrm{CoO}$ & $\mathrm{NiO}$ & $\mathrm{CuO}$ \\
\hline$E_{g}^{\text {theory }}[\mathrm{eV}]$ & & 3.59 & 2.5 & 2.66 & 3.9 & \\
$E_{g}^{\exp }[\mathrm{eV}]$ & 3.47 & $3.6-3.8$ & $?$ & $\sim 3$ & $4-4.3$ & 1.4 \\
\hline
\end{tabular}


material and the energy gap correction [133]. The band gap is then given by [133]:

$$
E_{g}(x, T)=E_{g}(Z n S e)+\Delta E \cdot x-b \chi T,
$$

where $\Delta E$ is the difference between the MnSe and ZnSe band gap, and $b$ is a parameter which is deduced from the second-order perturbation. Bylsma et al. noted, that this theory is only valid for small Mn concentrations $(x \leq 0.3)$. For high spin densities the energy correction is no longer proportional to $\psi$, which could be due to an increasing factor of higher perturbation terms as well as a change of magnetism from paramagnetic phase into a spin-glass phase [133]. This examples show, that the interaction between the d-shell states and conduction/valence band could not be neglected. Such anomalous band gap bowing was reported for transition metal alloyed $\mathrm{ZnO}$ [134], too. 


\section{Chapter 3}

\section{Preparation of transition metal alloyed $\mathrm{ZnO}$}

This chapter gives a short review of the fabrication of $\mathrm{ZnO}$ and $\mathrm{Zn}_{1-x} \mathrm{TM}_{x} \mathrm{O}$ single crystals, thin films and nanowires, and finishes with the preparation of ZnO:TM samples, which were characterized within this thesis. Thereby, it is necessary to avoid the formation of secondary phase clusters within the TM alloyed ZnO matrix $[15 ; 19 ; 20 ; 44 ; 135-137]$. The main goal is the successful optical and magnetical activation of $\mathrm{TM}$ in $\mathrm{ZnO}$.

\subsection{Bulk crystals}

Bulk $\mathrm{ZnO}$ single crystals were grown either by melt-growth technique or by hydrothermal growth with sizes up to three inch in diameter, whereas solid state reaction results in polycrystaline ZnO crystals [138-150].

By hydrothermal growth various substances could be crystallized from a high temperature aqueous solution at high vapor pressures [151]. The chemist Robert Bunsen used the hydrothermal growth for the first time in 1839 for the fabrication of barium carbonate and strontium carbonate at $200{ }^{\circ} \mathrm{C}$ and pressures above 100 bars [151]. These conditions mark the first use of hydrothermal aqueous solvents as media and was used later for the production of microscopic crystals by Schafhäult in 1845 and by de Sénarmont in 1851 [151]. Thereby, hydrothermal synthesis is a synthesis method for single crystals and depends on the solubility of minerals in hot water or mineralizers under high pressure [151]. Between the opposite ends of the growth chamber a temperature gradient is maintained, whereby the nutrient solution (marked with $\mathrm{N}$ in figure 3.1 ) is placed at the hotter end and the seed crystals $(\mathrm{S}$ ) 


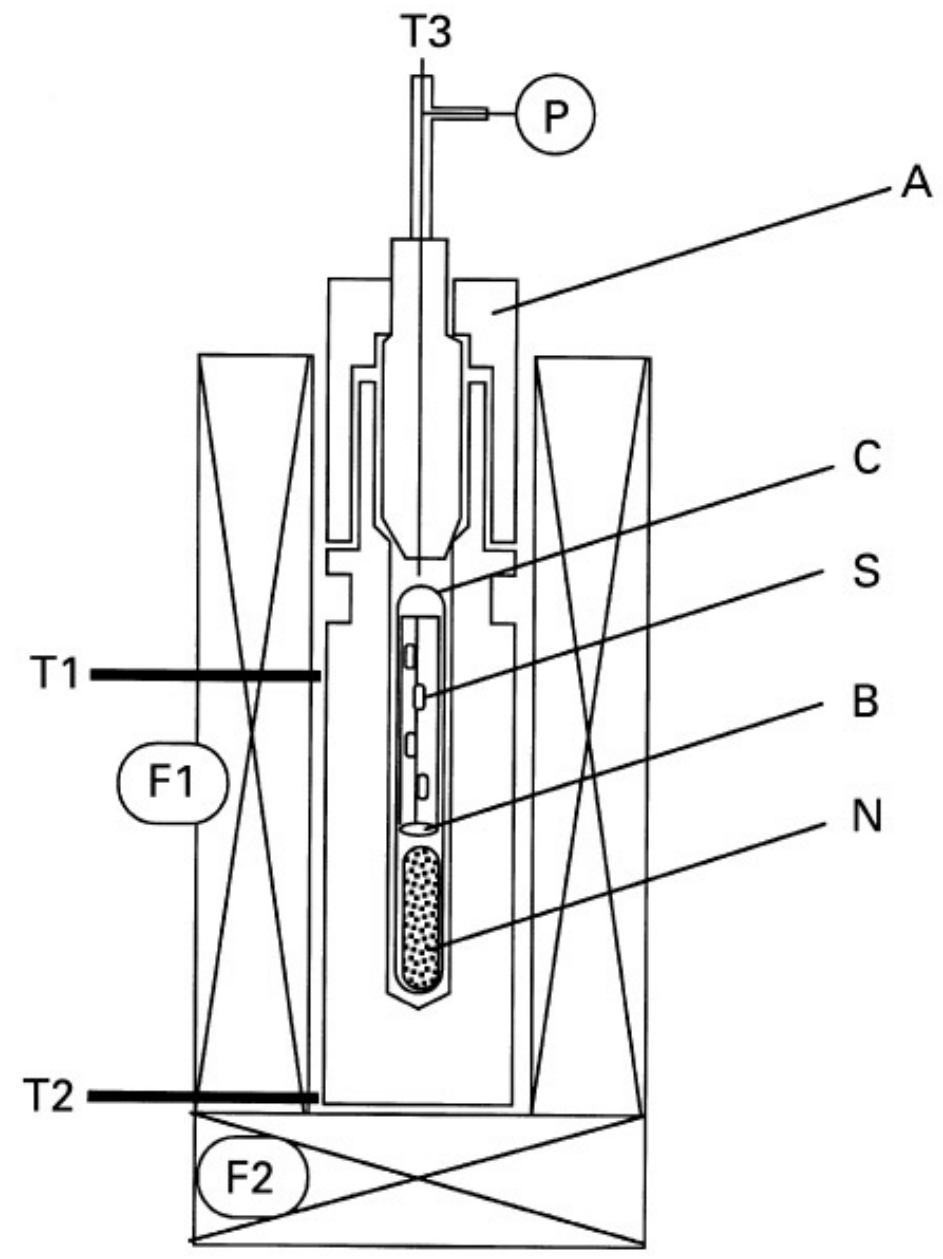

Figure 3.1: A hydrothermal growth system consists of two furnaces (F1 and F2), an autoclave and three thermocouples for temperature monitoring and control (T1 for furnace F1; T2 for furnace F2; and T3 within the autoclave) (image from [141]). Within the autoclave a Pt crucible (C) is placed which is filled with the nutrient (N) and seed crystals (S). (B) is a Pt baffle and (P) a pressure gauge.

at the cooler end $[141 ; 151]$. The hydrothermal method makes it possible to create crystalline phases which are not stable at the melting point or have a high vapour pressure near the melting point. Disadvantages of the method include the need of expensive autoclaves and good quality seeds of fair size [151].

High-purity $\mathrm{ZnO}$ reagent is pressed into tablets and sintered at $800-1000{ }^{\circ} \mathrm{C}$ for hydrothermal growth of $\mathrm{ZnO}$ single crystals [141; 142]. These $\mathrm{ZnO}$ tablets serve as starting nutrient and are placed in a Pt crucible together with a aqueous solution of potassium hydroxide $(\mathrm{KOH})$, lithium hydroxide $(\mathrm{LiOH})$ and ammonium hydroxide $\left(\mathrm{NH}_{4} \mathrm{OH}\right)[141 ; 142]$. Several $\mathrm{ZnO}$ seeds on a Pt wire are placed on top of the nutrient solution, whereas both are separated by a Pt baffle. The autoclave with the sealed crucible is put into a two-zone furnace system. A schematic setup is shown in figure 3.1 [141]. The system temperature is than raised to about $400{ }^{\circ} \mathrm{C}$, whereas the seed region temperature is set at about $8-15{ }^{\circ} \mathrm{C}$ lower than that of the nutrient region $[141 ; 142]$. The raising temperature produces a pressure up to $100 \mathrm{MPa}$. Growth 
rates in the [0001] direction of $0.15-0.2 \mathrm{~mm}$ per day could be achieved [142]. Li, K and $\mathrm{Na}$ are identified as main impurities within hydrothermal grown $\mathrm{ZnO}$ crystals from the aqueous solution and $\mathrm{Ti}, \mathrm{Fe}, \mathrm{Ca}$ and $\mathrm{Al}$ from the nutrient [141; 142].

All hydrothermal grown $\mathrm{ZnO}$ single crystals substrates, which were used within this thesis, were bought from CrysTec $\mathrm{GmbH}$. The $\mathrm{ZnO}$ substrates were cut from one $\mathrm{ZnO}$ crystal and subsequently polished on one side by the manufacturer.

For melt-grown ZnO single crystals a modified Bridgeman growth apparatus is used [146; 152-154], including a pressure vessel that contains pressurized oxygen from a source. A cooling unit is situated within the pressure vessel receiving the coolant flow from outside of the vessel and has cooled surfaces that define an enclosure [153]. The setup further includes an inductive heating element situated in the vessel and is coupled to a radio-frequency (rf) power-unit. $\mathrm{ZnO}$ powder is placed inside the cooling unit which might be optionally blended with dopand materials. A seed crystal of appropriate orientation can be placed inside the cooling unit. The interior portion of the $\mathrm{ZnO}$ is melted by the inductive heating element with a surrounding cooler solid-phase portion, that is cooled by the cooling outer unit [153]. The pressurized oxygen ambient could be mixed with an additional doping gaseous within the vessel [153]. The lowering of the cooling unit results in crystal nucleation at the base of the cooling unit and preferential crystal growth through the distance traveled [153]. After the growth process, the $\mathrm{ZnO}$ crystal is extracted from the cooling unit and is cut into wafer form, and processed by polishing and/or etchant to a predetermined or standard thickness.

Kane et al. show the possibility of the production of $\mathrm{Zn}_{1-x} \mathrm{Mn}_{x} \mathrm{O}$ and $\mathrm{Zn}_{1-x} \mathrm{Co}_{x} \mathrm{O}$ bulk single crystals by the melt-growth technique with a maximum concentration of 5 at.\% for $\mathrm{Mn}$ and 3 at.\% for Co in relation to Zn [154; 155], respectivelly. Thereby no secondary phase diffraction could be discovered within x-ray diffraction patterns. However, the presentation of ferrite single crystal growth by melt-growth might be a sign for the limitation of this technique [156]. And therefore, small segregations could be formed during the growth process which are too small enough to be undetectable by XRD.

Ceramic like pallets could be fabricated by the solid state reaction process [147150], where $\mathrm{ZnO}$ source powder is mixed together with a TM-oxide powder. The ratio of the mixture regulates the composition. These mixture is sintered at elevated temperatures between 500 to $1350{ }^{\circ} \mathrm{C}$. Such high TM alloyed ZnO ceramics reveal the formation of secondary phases $[148 ; 149]$. 


\subsection{Thin films}

Thin films of $\mathrm{ZnO}$ and $\mathrm{Zn}_{1-x} \mathrm{TM}_{x} \mathrm{O}$ are commonly grown by pulsed laser deposition [62; 157-171], sputtering [172-179], chemical solution deposition (sol-gel process) [180-185] or molecular beam epitaxy [186].

\subsubsection{Pulsed laser deposition}

Pulsed laser deposition (PLD) is a thin film deposition technique where a high power pulsed laser beam is focused inside a vacuum chamber to evaporate material from a target of the desired composition and deposited as thin film on a substrate (see figure 3.2) [187]. The physical phenomena of laser-target interaction and film growth are quite complex (form more details see reference [187-189] and references therein). The laser pulse is absorbed by the target and energy is first converted to electronic excitation and later on into thermal, chemical and mechanical energy, which result in evaporation, ablation and plasma formation [187]. The ejected species expand into the surrounding vacuum/ambient in the form of a plume containing many energetic species including atoms, molecules, electrons, ions and particles, before depositing on the typically hot substrate [187].

Pure $\mathrm{ZnO}$ is grown by PLD from a pure $\mathrm{ZnO}$ target, which was sintered from pure $\mathrm{ZnO}$ powder by the solid state reaction process (see section 3.1) [166-169], or from a pure Zn metal foil $[62 ; 170 ; 171]$. For the growth of TM alloyed ZnO, the PLD target are sintered with a defined TM concentration by intermixing ZnO powder with TM-oxide powder [166-169]. As alternative two different metal foils are used for TM:ZnO growth [170; 171], whereas one is a $\mathrm{Zn}$ foil and the other one is TM dopand foil. The laser is then screened over both foils. As for pure $\mathrm{ZnO}$ growth a partial oxygen pressure of some tenth mbar is applied and the substrates are heated to about $700{ }^{\circ} \mathrm{C}$. Thereby most work groups use a-plane $(11 \overline{2} 0)$ or c-plane (0001) sapphire as substrates. Transition metal concentrations of $x=0.3$ are obtained for $\mathrm{Zn}_{1-x} \mathrm{TM}_{x} \mathrm{O}$ (30 at.\% TM compared to Zn) [190-192], whereas especially highly TM alloyed $\mathrm{ZnO}$ shows the formation of secondary phases during the growth by PLD [162; 169; 192].

During this thesis PLD grown $\mathrm{ZnO}$ thin films were implanted with vanadium, these films were grown from a ceramic $\mathrm{ZnO}$ target. The $\mathrm{ZnO}$ films have a typical thickness of about $1 \mu \mathrm{m}$ and were grown on a-plane sapphire substrates at $650{ }^{\circ} \mathrm{C}$ with an oxygen partial pressure of about 0.002 mbar using a $\mathrm{KrF}$ laser with an energy density of $2 \mathrm{~J} / \mathrm{cm}^{2}$ [167]. A second set of PLD ZnO samples were grown for 


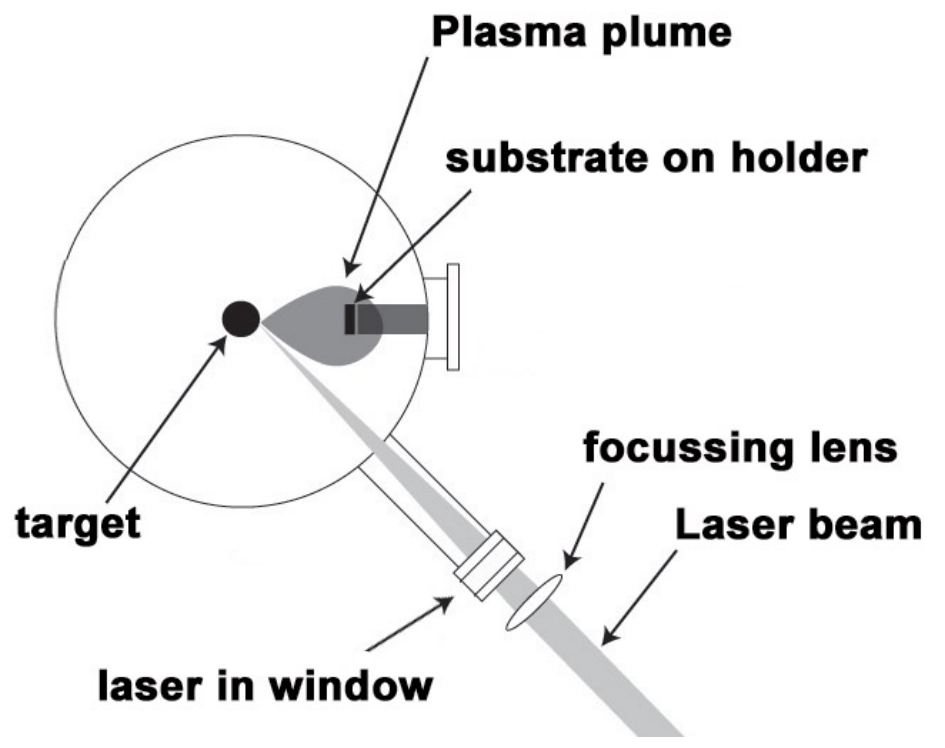

Figure 3.2: Schematic drawing of a pulsed laser deposition system: A laser beam is focused onto a target, where material is evaporated from the target and deposited on a substrate [187].

deep level transient spectroscopy measurements (see section 4.6) with an additional Al doped bottom layer with a thickness of about $200 \mathrm{~nm}$.

\subsubsection{Sputtering}

Sputtering is a process whereby atoms are ejected from a solid target material due to bombardment of the target by energetic ions and is commonly used for thin-film deposition, etching and analytical techniques [193]. Sputter deposition is a method of depositing thin films by sputtering material from a target which is then deposited onto a substrate. Atoms can be ejected from the target by momentum exchange between the sputtering ions and the target atoms, due to collisions [193]. The average number of atoms ejected from the target per incident ion is called the sputter yield. The sputtering yield from the target is thereby one of the main parameters for sputter deposition of thin films and depends on the ion incident angle, the energy of the ion, the masses of the ion and target atoms, and the surface binding energy of atoms in the target [193]. Sputtered atoms and ions ejected from the target have a wide energy distribution, whereas the sputtered ions can ballistically fly from the target in straight lines and impact energetically on the substrates or vacuum chamber (causing resputtering) [193]. However, the sputtered ions could also collide with gas atoms which act as moderator or as necessary growth reactant. Typically an inert gas is used for sputtering (for example argon or xenon).

Reactive sputtering is used to prepare oxide or nitride films by chemical reaction between the target material and the sputtering gas mixture which is introduced into 
the vacuum chamber [194-197]. Thereby the film composition could be controlled by varying the ratio of the gas mixture. Most work groups prepare $\mathrm{Zn}_{1-x} \mathrm{TM}_{x} \mathrm{O}$ thin films by reactive magnetron sputtering with a gas mixture of argon and oxygen [172178]. The ratio between zinc and transition metal dopand is controlled by the sputter ratio between both targets during co-sputtering $[173 ; 174 ; 176-178]$ or by the use of different $\mathrm{Zn}_{1-x} \mathrm{TM}_{x} \mathrm{O}$ ceramic targets $[172 ; 175 ; 179]$ during sputtering from only one target. During growth the substrate temperature is kept between $300-600{ }^{\circ} \mathrm{C}$ and c-plane sapphire, silicon or silicon oxide are used as substrates. By reactive magnetron sputtering $\mathrm{Zn}_{1-x} \mathrm{TM}_{x} \mathrm{O}$ thin fims were grown with $x=0.3[173 ; 174]$, whereby some studies reveal the formation of secondary phases $[173 ; 177]$.

\subsubsection{Sol-gel process}

The sol-gel process is a wet-chemical technique for the deposition of materials starting either from a chemical solution (sol for solution) or colloidal particles (sol for nanoscale particle) to produce an integrated network (gel) [198].

At first oxalate precursors are formed from metal alkoxides and/or metal chlorides, which undergo hydrolysis and polycondensation reactions to form these precursors (sol) [198]. The sol evolves then towards the formation of an inorganic continuous network containing a liquid phase (gel). The liquid phase from the gel is than removed by drying and forms a porous material, which is further treated by annealing to enhance the mechanical and structural properties [198]. The precursor sol can be either deposited on a substrate to form a film (e.g. by dip-coating or spin-coating), cast into a suitable container with the desired shape (e.g. to obtain a monolithic ceramics, glasses, fibers, membranes, aerogels), or used to synthesize powders (e.g. microspheres, nanospheres) [198].

$\mathrm{Zn}_{1-x} \mathrm{TM}_{x} \mathrm{O}$ is prepared by dissolving zinc acetate dihydrate $\left[\mathrm{Zn}\left(\mathrm{CH}_{3} \mathrm{COO}\right)_{2} \cdot 2 \mathrm{H}_{2} \mathrm{O}\right]$ and transition metal acetate tetrahydrate $\left[\mathrm{TM}\left(\mathrm{CH}_{3} \mathrm{COO}\right)_{2} \cdot 4 \mathrm{H}_{2} \mathrm{O}\right]$ in ethanol or diethylene glycol and is stirred for about one hour $[180 ; 184]$. The chelating reactions could be supported by the addition of acetylaceton as agent and hydrogen chloride $(\mathrm{HCl})$ as catalyst [180]. The solution is than deposited on a substrate by spin coating at room temperature and subsequently annealed in reduced oxygen atmosphere between 400 to $600{ }^{\circ} \mathrm{C}$ for $10 \mathrm{~min}$ by rapid thermal annealing [180; 182]. All deposited thin films are polycrystalline and show the incorporation of secondary phases [180; 182]. 


\subsection{Nanowires}

Typically, $\mathrm{ZnO}$ nanowires are grown via a vapor transport technique or wet chemical growth (sol-gel) [39; 199-203], whereas the vapor transport technique exploits the vapor-liquid-solid growth process. On the other hand TM-O (with TM $=\mathrm{Fe}, \mathrm{Mn}$, $\mathrm{Ni}$ or Co) are commonly fabricated by wet chemical processes (sol-gel) [204-217], whereas up to now only one report deals with the formation of $\mathrm{MnO}$ nanowires by a vapor transport technique [218]. However, during the the vapor transport growth of $\mathrm{MnO}$ nanowires also $\mathrm{Mn}_{3} \mathrm{O}_{4}$ nanowires are produced [218]. Hence, the wet chemical growth method seems to be the perfect method for the production of $\mathrm{Zn}_{1-x} \mathrm{TM}_{x} \mathrm{O}$ nanowires, but the sol gel method is also used for growth of $\mathrm{TMO}_{2}, \mathrm{TM}_{2} \mathrm{O}_{3}$ and $\mathrm{TM}_{3} \mathrm{O}_{4}$ nanowires $[217 ; 219-223]$. Therefore, controlled doping of $\mathrm{ZnO}$ with TM during growth seems not easy. During wet chemical growth the dopant species could form easily aquo ions which could not be incorporated into the crystal lattice [203]. This problem is solved by adding transition metal acetate into the solution but yield the formation of elemental secondary phases. Those secondary phase could be removed by further treatment in $\mathrm{HCl}$ acid [201], resulting in a further preparation step. It is possible to add acids directly during the wet chemical preparation of $\mathrm{ZnO}$ nanowires. The resulting change of the ph-value of the solution affects the resulting morphology [203] and needs an accurate control of the solution.

Growth of transition metal doped $\mathrm{ZnO}$ nanowires by the vapor transport technique encounters comparable problems, like the formation of secondary phase oxides [44; 224] or an insufficient control of the doping concentration of a $\mathrm{ZnO}$ nanowire $[225 ; 226]$. Due to the high growth temperature the transition metals sources could be quite simply oxidized, especially if the oxygen partial pressure is high. As a result the transition metal fraction in the vapor and therefore in the $\mathrm{ZnO}$ nanowire is reduced. The addition of transition metal and/or transition metal oxide vapor during the growth changes the conditions within a catalytic process such as vapor-liquidsolid growth.

\subsubsection{Vapor-liquid-solid process}

The vapor-liquid-solid (VLS) growth process was describet in 1964 by Wagner and Ellis for the growth of single crystalline silicon rods with diameters from $100 \mathrm{~nm}$ up to $0,2 \mathrm{~mm}$ [41]. The salient feature of the VLS growth is a small sphere like head on top of every nanorod or nanowire [41;228], which acts as catalyst during the growth. The catalyst is deposited prior the growth on a substrate as thin film 

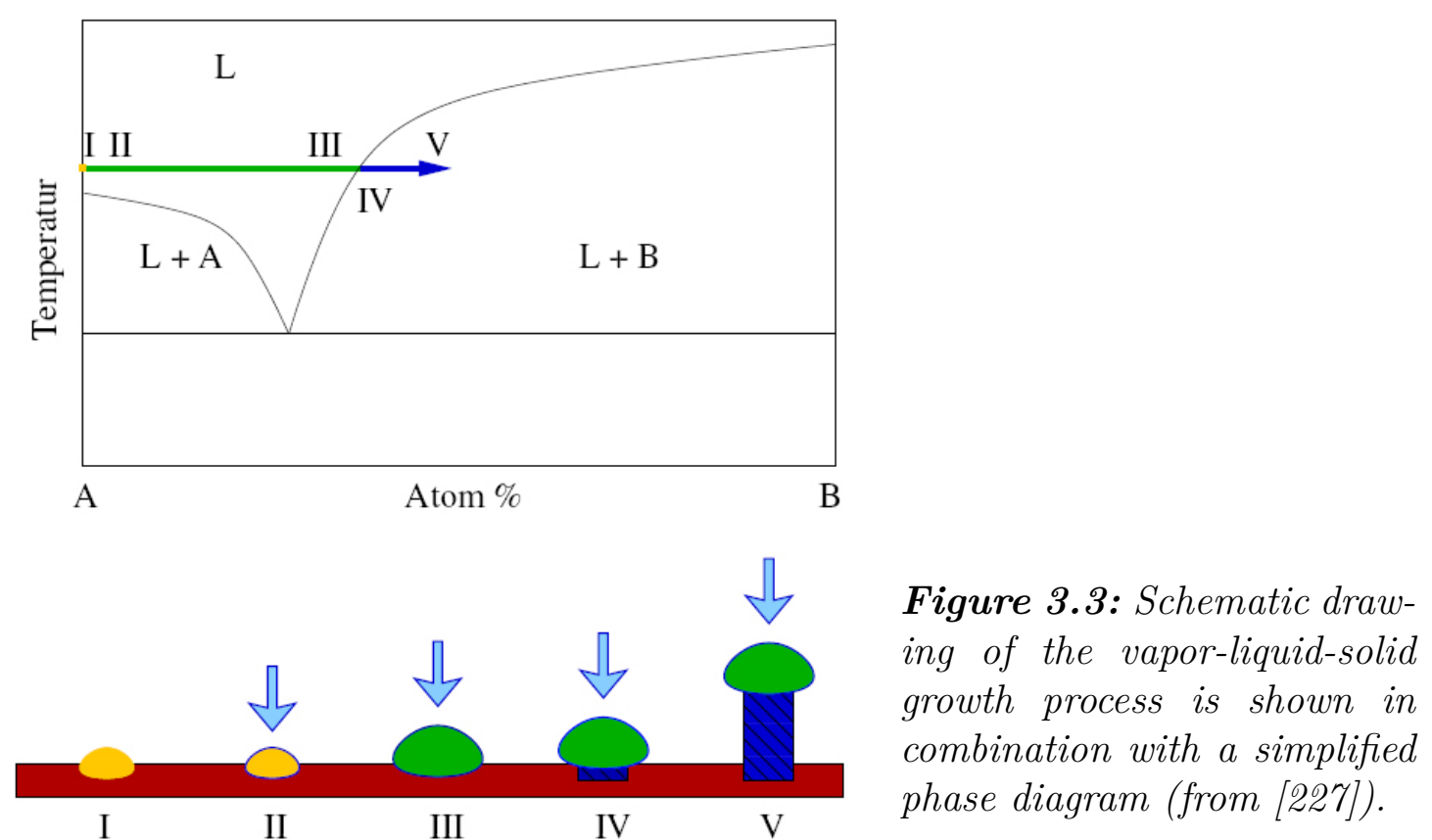

or from a metal colloide [39;229-231], whereas the size of the catalyst particles controls the diameter of the grown nanowires. The process of the VLS growth is shown in figure 3.3 with a schematic demonstration of the growth process within a simplified phase diagram. The substrate with the catalyst is heated near or above the melting point of the catalyst (I). The source vapor (B) is condensed on the catalyst (A) particle which forms a liquid alloy of the catalyst material and the source vapor (II). With the time the concentration of the source material within the liquid alloy increases and the composition of the liquid alloy moves further to the right side of the phase diagram to point (III). In point (IV) the liquid alloy reaches a critical concentration of the source material (B), the alloy supersaturates and forms a solid nucleus on the interface to the substrate. Consecutively with time, material is deposited on the nucleus which forms a small rod with further increasing length during the growth and a sphere like catalyst aquaplaning on top of the nanowire (see high resolution transmission electron microscopy image in figure 3.4a [40]). The length depends thereby on the growth duration, at which the source materials is provided. Successful VLS growth needs an eutectic in the respective alloy system with a very small mutual solubility in the solid state, that is given for the binary phase diagrams of the $\mathrm{Au}-\mathrm{Si}$ and $\mathrm{Au}-\mathrm{Ge}$ systems [41; 232].

For the synthesis of compound semiconductors nanowires ( $\mathrm{ZnO}, \mathrm{ZnS}$ and GaAs) a pseudobinary phase diagram is treated for the VLS growth of these materials [233], if the compound semiconductor material is immiscible with the catalyst. In geological 

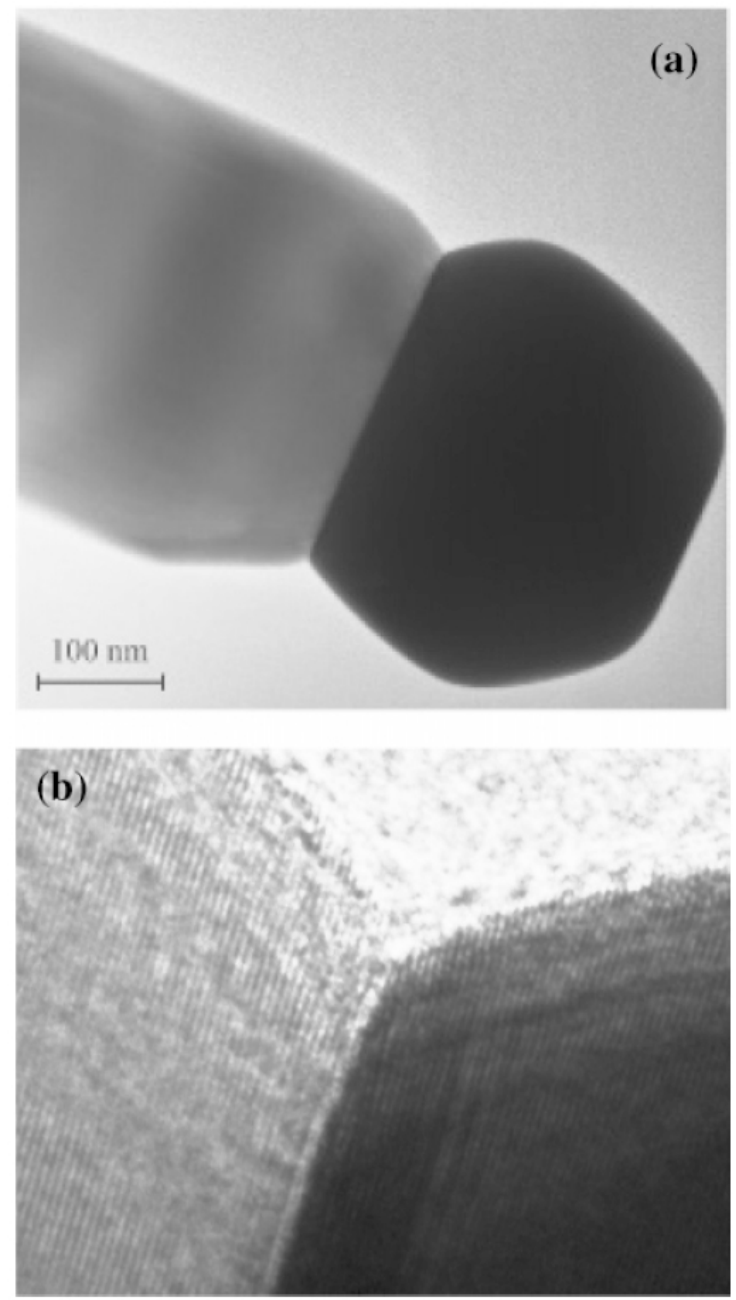

Figure 3.4: HR-TEM micrograph of the interface between a $\mathrm{ZnO}$ nanostructure and the Au catalyst after growth. The lattice fringes of both two phases, $\mathrm{ZnO}$ and $\mathrm{Au}$, are parallel to each other and match the lattice spacing of $\mathrm{ZnO}$ (0002) [ 0.30 nm] and $\mathrm{Au}$ $\{110\}[\sim 0.28 \mathrm{~nm}]$ (see [40]). ).

studies of the Zn-Au mineralization $\mathrm{ZnS}$ was found as sphalerite (cubic $\mathrm{ZnS}$ ) and $\mathrm{Au}$ as pure metal, whereas $\mathrm{Fe}, \mathrm{Cd}, \mathrm{Sb}$, and $\mathrm{Cu}$ are found as solutes in sphalerite, but no $\mathrm{Au}$ [234]. EDS measurements on ZnS nanowires capped with an Au dot on top of the wire reveal no Au signal from the $\mathrm{ZnS}$ nanowire and vice versa no signal from $\mathrm{Zn}$ and sulfur from the $\mathrm{Au}$ dot [235]. This observation was made for $\mathrm{ZnO}$ nanowires capped with an $\mathrm{Au}$ dot as well [236], which shows the chemical similarity of the ZnS/Au combination to the system $\mathrm{ZnO} / \mathrm{Au}$. The immiscibility is also shown in figure 3.4, where (a) shows the bright $\mathrm{ZnO}$ nanowire with a dark Au droplet on top. Figure $3.4 \mathrm{~b}$ is a close-up of the interface and reveal the lattice fringes of the two phases $(\mathrm{ZnO}$ and $\mathrm{Au})$ which are perfectly parallel to each other. The lattice spacing in the $\mathrm{ZnO}$ is $(0.30 \pm 0.03) \mathrm{nm}$, corresponding to $\mathrm{ZnO}(0002)$. The lattice spacing in the $\mathrm{Au}$ droplet is $(0.28 \pm 0.03) \mathrm{nm}$, corresponding to the forbidden $\mathrm{Au}\{110\}$. Thereby, no evidence of a eutectic microstructure can be detected. In XRD investigations, the $\mathrm{ZnO}(0002)$ lattice spacing was established as $(0.26 \pm 0.01) \mathrm{nm}[237]$. 

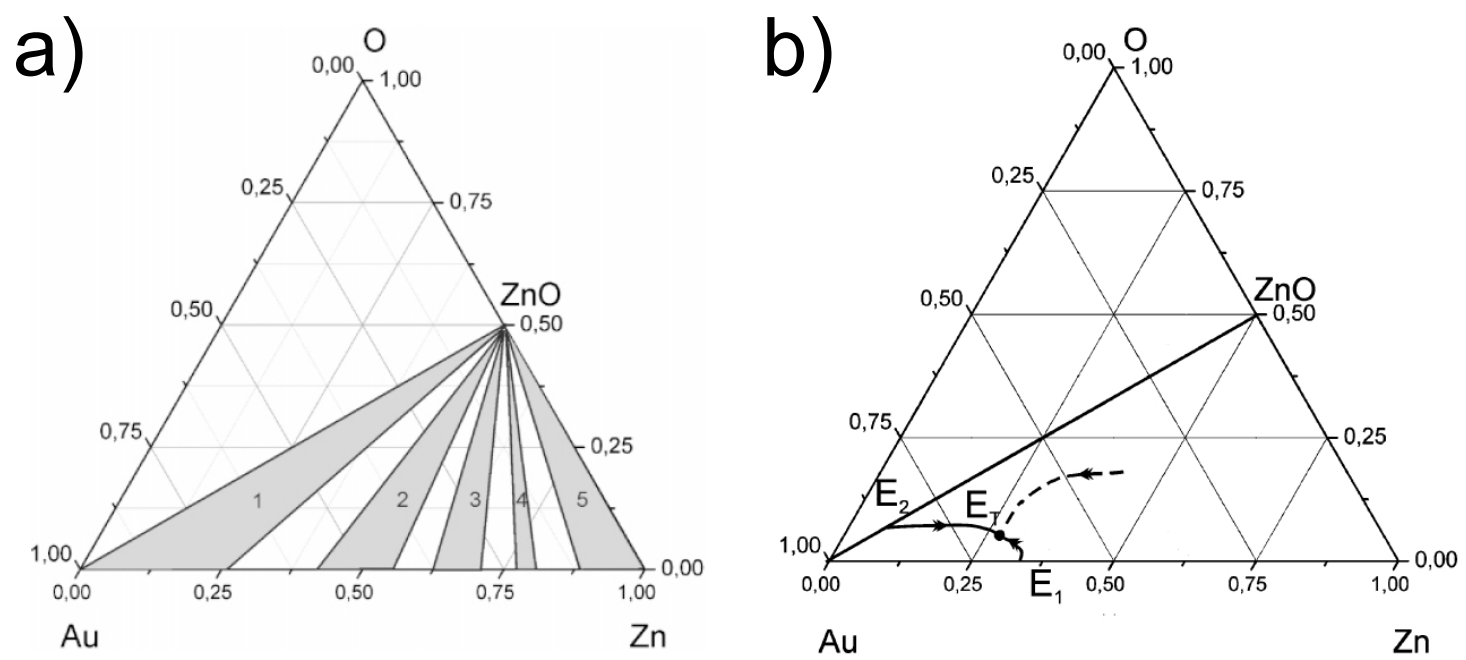

Figure 3.5: A) shows an isothermal section of a tentative ternary Au-Zn-O phase diagram at about $550{ }^{\circ} \mathrm{C}$ (see [40]), whereas two-phase regions are gray: (1) $(A u)+Z n O$, (2) $A u Z n+Z n O$, (3) $A u Z n_{3}+Z n O$, (4) $A u Z n_{4}+Z n O$, and (5) $L+Z n O$, where $L$ is the $Z n$-rich liquidus. The white regions are three-phase regions. B) Tentative liquidus surface of the proposed phase diagram. $E_{1}$ : eutectic $L \leftrightarrow(A u)+A u Z n$ at $683^{\circ} \mathrm{C}$ and 33.5 at.\% $\mathrm{Zn}$. $E_{2}$ : quasibinary eutectic between $A u$ and $Z n O: L \rightleftharpoons(A u)+Z n O$ at $650^{\circ} C<T<1000^{\circ} C$. $E_{T}$ : ternary eutectic $L \rightleftharpoons(A u)+Z n O+A u Z n$ at $T \leq 650^{\circ} C$ (see [40]).

The temperature of the source material $\mathrm{ZnO}$ is heated to $1350{ }^{\circ} \mathrm{C}$ during the growth of $\mathrm{ZnO}$ nanowires. It was shown by mass spectrometric intensities during the vaporization of $\mathrm{ZnO}$, that the vapor pressure of $\mathrm{ZnO}$ is about three orders of magnitude lower than the vapor pressure of $\mathrm{Zn}$ and $\mathrm{O}_{2}$ at about $1350{ }^{\circ} \mathrm{C}$ [238]. Therefore, the use of a pseudobinary phase diagram is unsatisfactory, whereas no ternary phase diagram of the $\mathrm{Au}-\mathrm{Zn}-\mathrm{O}$ system is known. To our knowledge no ternary solid phase has been reported for Au-Zn-O. Figure 3.5a shows a tentative ternary phase diagram [40], whereby it should be mentioned that no binary diagram of the $\mathrm{Au}-\mathrm{O}$ and Zn-O system is known. The miscibility of Au with oxygen is rather small (smaller than $10^{-4}$ in the liquidus as well as in the solid state phase) and only a intermediate high pressure phase $\mathrm{Au}_{2} \mathrm{O}_{3}$ exists. It is assumed that an equilibrium of unknown type exists in which the liquid and solid Au-rich solution is involved near the pure concentration of $\mathrm{Au}$ and melting point of $\mathrm{Au}\left(1064{ }^{\circ} \mathrm{C}\right)$ [40]. The Zn-O system looks similar: a ternary reaction of $L+(Z n)+Z n O$ near the melting temperature of pure $\mathrm{Zn}\left(420^{\circ} \mathrm{C}\right)$ at concentrations near pure $\mathrm{Zn}$ is assumed with an additional ternary reaction near the concentration of pure $\mathrm{ZnO}$. The binary phase diagram of the Au-Zn system is well know (see figure 3.6 [239]) and shows numerous 


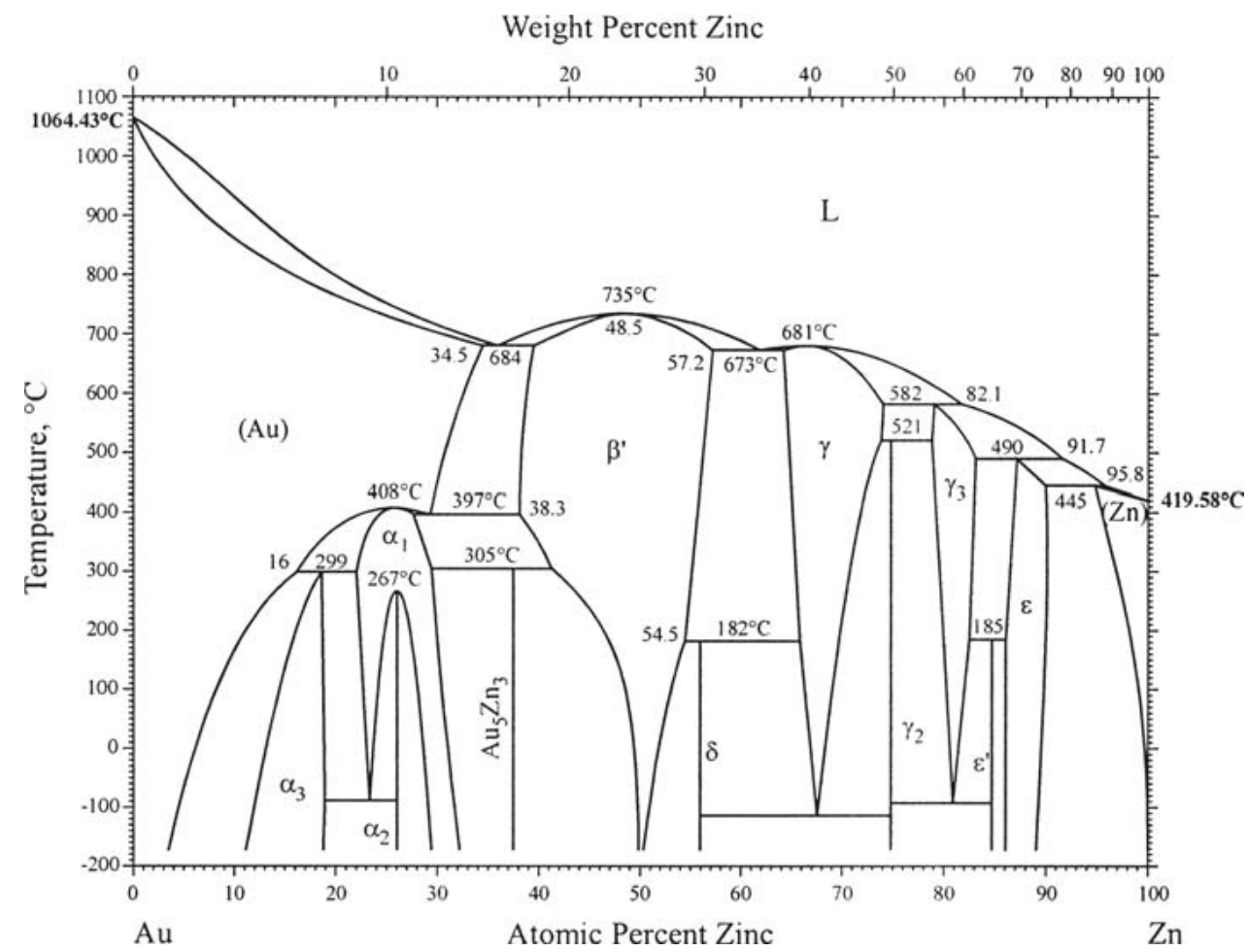

Figure 3.6: The figure shows the binary phase diagram of Au-Zn (from [239]).

intermetallic phases, and two eutectic points. The eutectic point on the Au-rich side is located at $683{ }^{\circ} \mathrm{C}$ with an Zn concentration of 33.5 at. $\%: L \leftrightarrow(A u)+A u Z n$, whereas $A u Z n$ has a melting point of $753{ }^{\circ} \mathrm{C}$. Figure 3.5 a shows a proposed isothermal section of a ternary Au-Zn-O phase diagram at about $550{ }^{\circ} \mathrm{C}$, whereas the quasibinary system $\mathrm{Au}-\mathrm{ZnO}$ divides the system into two parts: $\mathrm{Au}-\mathrm{ZnO}-\mathrm{O}$ and $\mathrm{Au}-$ ZnO-Zn. Due to the Zn-rich growth conditions of the VLS process [39; 240], only the Au-ZnO-Zn part will be considered further. The gray and market zones in figure 3.5a are two-phase regions: (1) $(A u)+Z n O,(2) A u Z n+Z n O,(3) A u Z n_{3}+Z n O$, (4) $A u Z n_{4}+Z n O$, and (5) $L+Z n O$, where $L$ is the Zn-rich liquidus. The extensions of the two-phase regions can be derived from the binary Au-Zn phase diagram [239]. Thereby the three-phase region (white zones are three-phase regions) between the two-phase region 1 and 2 in figure 3.5a is of special interest. This treephase region is composed of: $(A u)+Z n O+A u Z n$. From the binary eutectic point at 33.5 at\% $\mathrm{Zn}$, a ternary melting path must lead to a ternary eutectic equilibrium: $L \rightleftharpoons(A u)+Z n O+A u Z n$ at $T \leq 650{ }^{\circ} \mathrm{C}$, whereas a second melting path must lead to this eutectic point from a quasibinary eutectic between $\mathrm{Au}$ and $\mathrm{ZnO}$ : $L \rightleftharpoons(A u)+Z n O$ at $650{ }^{\circ} \mathrm{C}<T<1000{ }^{\circ} \mathrm{C}$. This situation is illustrated in fig- 


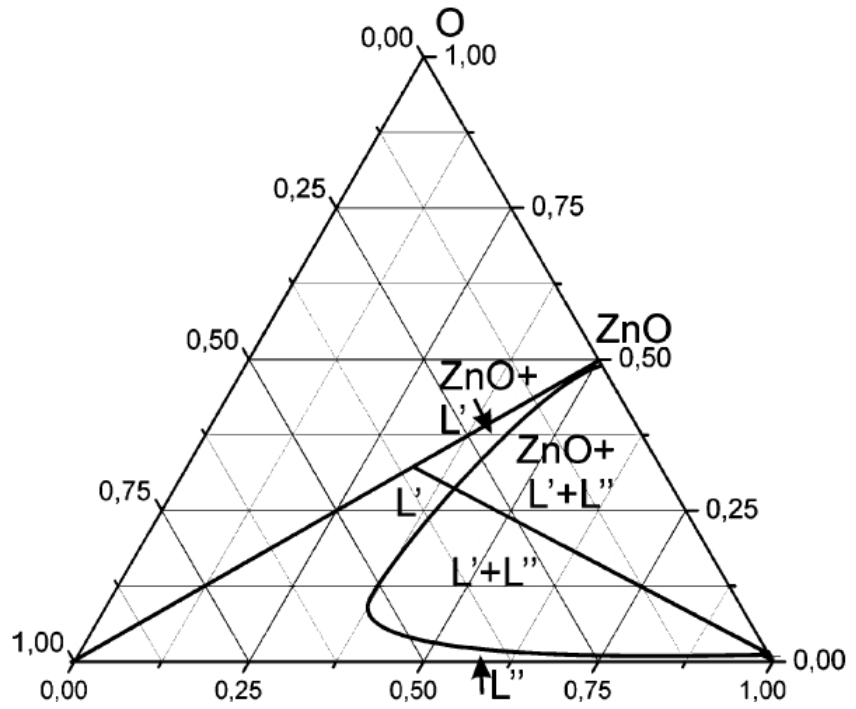

$\mathrm{Au}$
$\mathrm{Zn}$
Figure 3.7: Tentative isothermal section at $1100^{\circ} \mathrm{C}$ of the proposed phase diagram. (see [40]).

ure $3.5 \mathrm{~b}$ which shows the tentative liquidus surface of the proposed $\mathrm{Au}-\mathrm{Zn}-\mathrm{O}$ phase diagram.

Figure 3.7 shows a tentative isothermal section at $1100{ }^{\circ} \mathrm{C}$ of the proposed ternary system. With increasing zinc and oxygen content within the liquid Au droplet the Au-rich liquidus $L$ branches into ZnO-rich $L^{\prime}$ and $\mathrm{Zn}$-rich $L^{\prime \prime}$, due to the immisibility of $\mathrm{Zn}$ with $\mathrm{ZnO}$ [40]. There is a two-phase field $Z n O+L^{\prime}$ near $\mathrm{ZnO}$ and a three-phase field $Z n O+L^{\prime}+L^{\prime \prime}$ between $\mathrm{ZnO}$ and $\mathrm{Zn}$. The location of the critical point of the Au-rich liquidus in the phase diagram is purely hypothetical, the same applies to the solidus between $\mathrm{ZnO}$ and $L^{\prime}$. During growth the liquid alloy proceeds from pure $\mathrm{Au}$ toward $\mathrm{ZnO}$ into the $L^{\prime}$ branch and eventually supersaturates. Than $\mathrm{ZnO}$ is segregated in solid form from the melt which takes place as long as the temperature is held and $\mathrm{Zn}$ and $\mathrm{O}_{2}$ vapor is supplied to the melt. During cooling the liquidus turns Au-rich and solidifies as Au droplet. As long as excess zinc is available some material can remain molten, but the melt cannot take up any oxygen. During the cooling, Zn is still in the atmosphere, so the ternary eutectic ET can be reached below $650{ }^{\circ} \mathrm{C}$, where, if equilibrium is given, the melt must solidify to ( $\mathrm{Au}$ ), $\mathrm{ZnO}$, and AuZn. From literature it is know, that ternary eutectics degenerate to only two of three phases [241], and therefore only two phases could be observed.

In comparison to the $\mathrm{Au}-\mathrm{Zn}-\mathrm{O}$ system, where only a phase diagram for $\mathrm{Au}-\mathrm{Zn}$ exists, TM-O phase diagrams are well known [242-248]. Other than for Ni and Zn, all these phase diagram reveal the existence of many different TM-oxide phases with chemical composition such as TMO; $\mathrm{TM}_{2} \mathrm{O}_{3}$; and $\mathrm{TM}_{3} \mathrm{O}_{4}$, which are indicated 

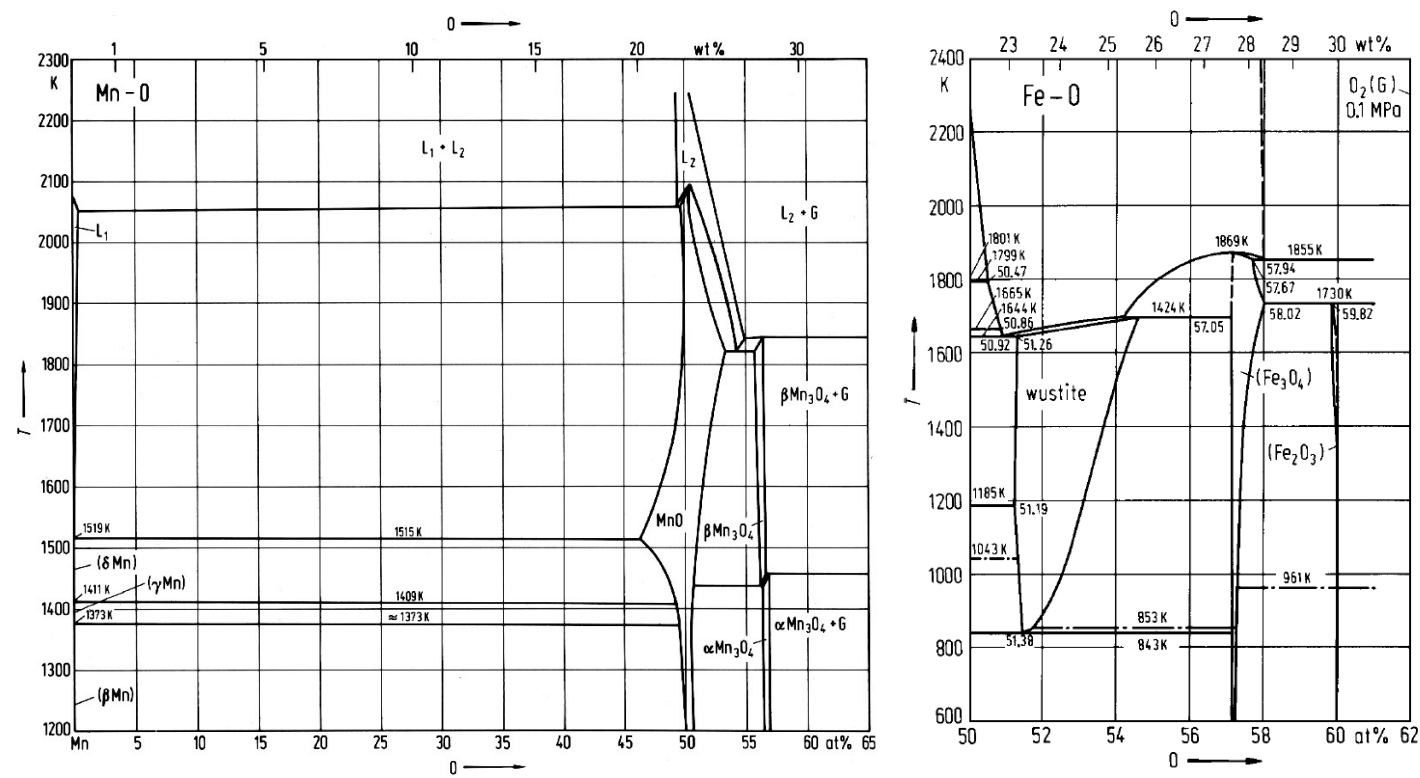

Figure 3.8: The left hand side shows the phase digram of the Mn-O system and the right hand side the phase diagram of the Fe-O system [242; 243]. Both digrams reveal the formation of different manganese oxides and iron oxides.

in figure 3.8 for the $\mathrm{Mn}-\mathrm{O}$ and Fe-O systems [242; 243]. As visible in figure 3.8 all these oxide phases are arranged side by side within the phase diagrams. Thereby the $\mathrm{Mn}-\mathrm{O}$ phase diagram is not complete, for higher oxygen concentrations also $\mathrm{MnO}_{2}$ and $\mathrm{Mn}_{2} \mathrm{O}_{3}$ exists [244]. It should be noted that some of these phases appear with different crystal structures. One other point is the high melting point of pure TM and TM-oxides, for all these systems the melting point is higher than the melting point of $\mathrm{Au}$ [249-252]. The high melting point and the number of different TM-oxide phases shows the complexity of the parameter space for the controlled growth of $\mathrm{Zn}_{1-x} \mathrm{TM}_{x} \mathrm{O}$ nanowires. Thereby, the temperature and source vapor ratios, especially the oxygen fraction, would be the main key factors for a controlled and successful growth.

\subsubsection{High temperature growth of $\mathrm{ZnO}$ nanowires}

The vapor transport technique is used for the growth of $\mathrm{ZnO}$ nanowires via the VLS growth process. As source 2 g pure $\mathrm{ZnO}$ powder (99\%, Merck) is placed on a sliced alumina tube as visible in figure 3.9, which is deposited in the center of a tube furnace. The $\mathrm{ZnO}$ nanowires are grown on p-type silicon (100) substrates which are coated with a $4 \mathrm{~nm}$ thick Au film. The Au coated substrates are placed on a second 


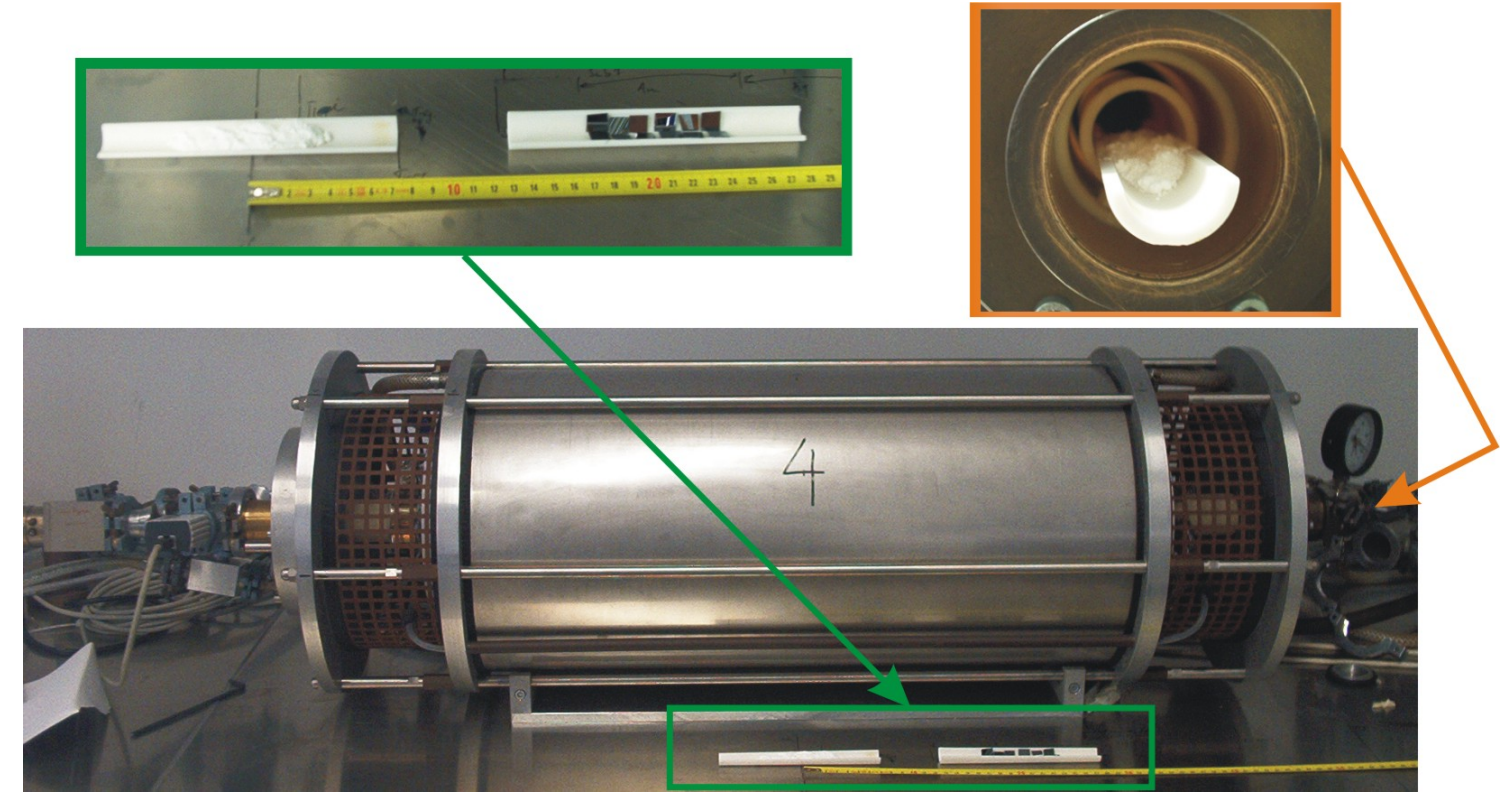

Figure 3.9: In front of the high temperature furnace two sliced alumina tubes are aligned, as they are placed during the growth in the furnace tube. The left one is filled with $\mathrm{ZnO}$ source powder and the right one with Au coated silicon substrates.

sliced alumina tube and placed behind the souce material in the furnace. The furnace is than closed and slowly evacuated to a base pressure of about $10^{-2}$ mbar. Figure 3.9 shows the self-made high temperature furnace which can be heated up to $1500{ }^{\circ} \mathrm{C}$ and is controlled via a programmable controller unit . The furnace is typically heated up to $1350{ }^{\circ} \mathrm{C}$ with a ramp of $190{ }^{\circ} \mathrm{C}$ per hour and kept at $1350{ }^{\circ} \mathrm{C}$ for $30 \mathrm{~min}$. An argon gas flow of $50 \mathrm{sccm}$ with a system pressure between 100-200 mbar was applied as transporting gas during the entire process. However, the flow direction was changed during the growth process. Only during the growth at $1350{ }^{\circ} \mathrm{C}$ the argon gas flow direction was adjusted to transport the $\mathrm{ZnO}$ vapor from the source to the substrate. An opposite gas flow direction was applied during the heating-up and cooling-down phases.

Figure 3.10a shows a scanning electron microscopy micrograph of an as-grown $\mathrm{ZnO}$ nanowire sample. Our samples are covered with triangular shaped belts and nanowires with a huge aspect ratio. The $\mathrm{ZnO}$ nanowires have a mean diameter of about $150 \mathrm{~nm}$ with an length up to $100 \mu \mathrm{m}$. Nanowire growth could only observed in the temperature range between 960 to $1160{ }^{\circ} \mathrm{C}$, and grow typically along the $\mathrm{ZnO}$ c-axis. Further details of the high temperature growth of $\mathrm{ZnO}$ nanostructures are presented in reference [237]. 


\subsection{Ion implantation and annealing}

In contrast to all this direct growth techniques ion implantation is used to prepare transition metal alloyed $\mathrm{ZnO}$ thin films within single crystal substrates, $\mathrm{ZnO}$ thin films or nanowires $[16-18 ; 47-49 ; 186 ; 253-262]$. By ion implantation the concentration and distribution could be controlled via the ion energy and ion fluence. It overcomes the obstacles of all growth techniques, especially the above mentioned doping difficulties of nanowires. Therefore, $\mathrm{ZnO}$ nanowires are prepared via a high temperature growth technique with the vapor-liquid-solid growth process (see section 3.3.2). Ion implantation is not limited to solubility limits of elements within the desired matrix in contrast to doping during growth or thermal in-diffusion. Even elements, which are not soluble within the desired matrix, could be introduced.

The kinetic energy of an impinging ion is transfered by nuclear and electronic interaction into the target host lattice. During nuclear collusion the nucleus of the impinged ion interacts with the nuclei of the target atoms. Whereas in electronic collusion the ion energy is transfered to electronic system of the target atoms. The relative importance of nuclear and electronic stopping depends on the ion energy, whereby the nuclear stopping energy is dominant for small ion energies and the electronic stopping for high ion energies [263]. If the transfered energy from the ion to a lattice atom is large enough, than a lattice atome is knocked out of its lattice side. This target atom then itself becomes a projectile in the solid which could generated further defects. Thus one impinging ion could generated hundreds of lattice defects, such as vacancies, interstitial atoms or atoms on antisites [263; 264]. The range and depth distribution of an implanted ion is represented by a Gauss distribution and depends on the ion mass, ion energy and the composition of the host crystal. The range and depth distributions is calculated with SRIM/TRIM [265], whereas for every transition metal element as set of ion energies was calculated to create boxlike implantation profiles. Figure $3.10 \mathrm{~b}$ shows a simulated box-like depth profile of Fe in $\mathrm{ZnO}^{1}$. As grown $\mathrm{ZnO}$ nanowire samples and CrysTec $\mathrm{ZnO}$ single crystal substrate were implanted with transition metal ions and the argon implanted reference series. Table 3.1 shows the used ion energies for the appropriate transition metal element with the overall ion fluences and the corresponding transition metal concentration. For reference argon reference series are made, whereas the ion implantation profile (energies) and ion fluences are adopted from the Fe implantation. All samples were implanted at room temperature.

The heavy ion $500 \mathrm{kV}$ implanter IONAS was used for all ion implantation experi-

${ }^{1}$ For the calculation a $\mathrm{ZnO}$ density of $5,6 \mathrm{~g} / \mathrm{cm}^{3}$ was assumed. 

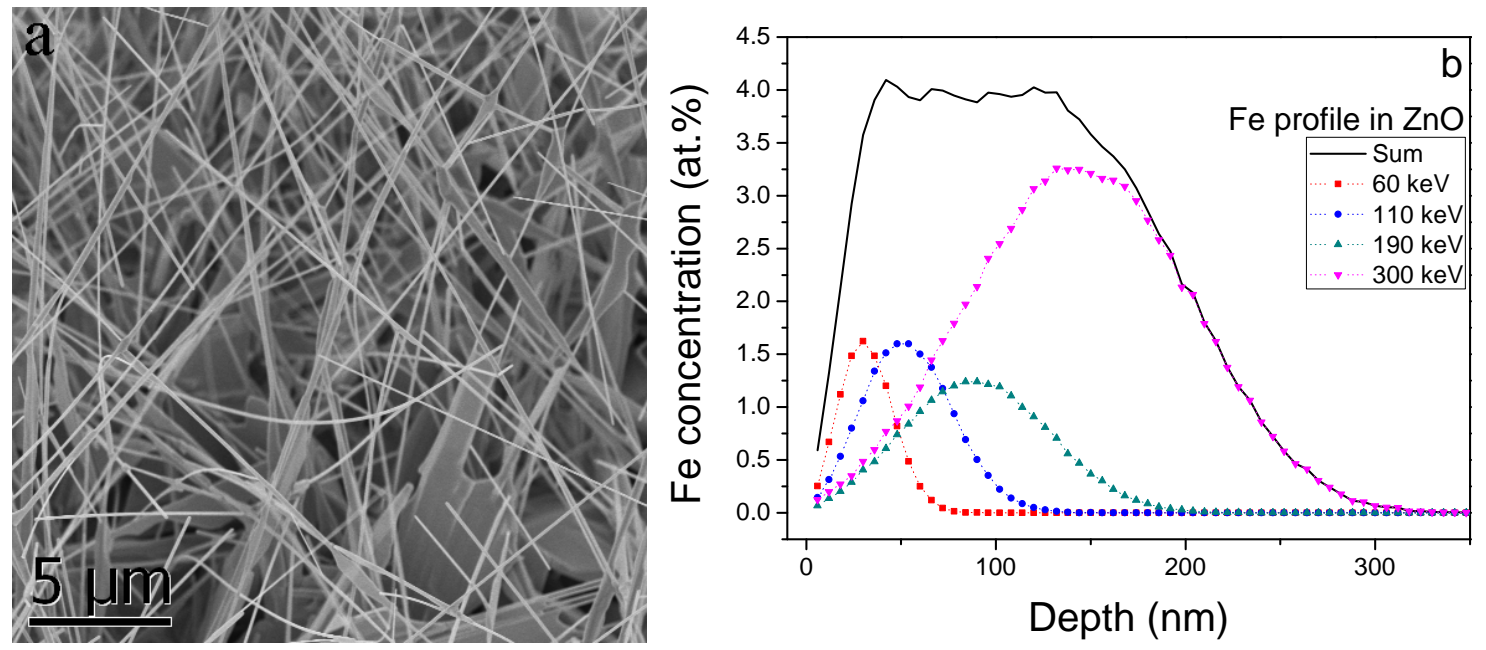

Figure 3.10: a) shows a scanning electron microscopy micrograph of an representative $Z n O$ nanowire sample which are used for ion implantation of transition metals. b) shows a box-like ion implantation profile of $\mathrm{Fe}$ in $\mathrm{ZnO}$ which is generated by a set of four different ion energies and is calculated with SRIM/TRIM [265].

ments [266]. IONAS is a open-air Cockcroft-Walton type implanter. The acceleration unit is protected in a separated cabin to maintain the a constant temperature of $20{ }^{\circ} \mathrm{C}$ and relative humidity of $40 \%$ for stable operation at $500 \mathrm{kV}$. The implanter is

Table 3.1: Energies, fluences and the corresponding transition metal concentration used within this thesis. For ${ }^{*}$ marked series PLD thin films are used, which are annealed in oxygen ambient at $800^{\circ} \mathrm{C}$ for 30 minutes. For the Ar reference samples of this series we chose the same ion energy and ion fluence as for the 5 at.\% V implanted sample.

\begin{tabular}{lrrr}
\hline Element & $\begin{array}{r}\text { Energy range } \\
{[\mathrm{keV}]}\end{array}$ & $\begin{array}{r}\text { Overall fluence } \\
{\left[\mathrm{cm}^{-2}\right]}\end{array}$ & $\begin{array}{r}\text { TM concentration } \\
\text { at. } \%\end{array}$ \\
\hline $\mathrm{V}^{*}$ & $30-250$ & $1.46 \cdot 10^{16}-8.76 \cdot 10^{16}$ & $0.83-5$ \\
$\mathrm{~V}$ & $65-300$ & $1.7 \cdot 10^{16}-6.8 \cdot 10^{16}$ & $1-4$ \\
$\mathrm{Mn}$ & $50-450$ & $2.6 \cdot 10^{15}-4.1 \cdot 10^{17}$ & $0.1-16$ \\
$\mathrm{Co}$ & $65-450$ & $2.6 \cdot 10^{15}-4.1 \cdot 10^{17}$ & $1-16$ \\
$\mathrm{Fe}$ & $60-300$ & $1.6 \cdot 10^{15}-2.51 \cdot 10^{17}$ & $0.1-16$ \\
$\mathrm{Ni}$ & $65-300$ & $1.6 \cdot 10^{16}-2.53 \cdot 10^{17}$ & $1-16$ \\
$\mathrm{Ar}$ & $60-300$ & $1.6 \cdot 10^{15}-1.26 \cdot 10^{17}$ & $1-8$ \\
\hline
\end{tabular}


equipped with a Sidenius type hot-filament hollow-cathode ion source, whereas the ions are extrected from the source with an acceleration voltage of 10, 20 or $30 \mathrm{kV}$. After mass separation in a $90^{\circ}$ double focusing magnet the ions are passed into the second acceleration stage with an maximum acceleration voltage of $500 \mathrm{keV}$. Four beamlines are attached on the ion implanter IONAS, whereas three were used for material analysis and one is for ion implantation/irradiation.

After ion implantation all samples were annealed in air to remove the majority of implantation defects. Prior every annealing step all samples were cleaned, whereat nanowire sample were cleaned by careful movement of sample back side over acetone impregnated paper. Single crystals were cleaned in boiling acetone, afterwards in boiling isopropanol and were subsequently dried under nitrogen gas flow. High dose (TM concentration above 4 at.\%) implanted $\mathrm{ZnO}$ single crystal samples were annealed stepwise at 100,300 and $500{ }^{\circ} \mathrm{C}$ for $15 \mathrm{~min}$ and for $30 \mathrm{~min}$ at 700 and $900{ }^{\circ} \mathrm{C}$. All other $\mathrm{ZnO}$ single crystal samples were only annealed for $30 \mathrm{~min}$ at 700 and $900{ }^{\circ} \mathrm{C}$ and all implanted nanowires sample at 30 min at $700{ }^{\circ} \mathrm{C}$. 


\section{Chapter 4}

\section{Characterization techniques}

This chapter introduces the main characterization techniques which were applied to transition metal implanted $\mathrm{ZnO}$ nanowires and single crystals. The structural impact and the formation of secondary phases of the ion implantation and annealing were analyzed by scanning electron microscopy and transmission electron microscopy (see section 4.1), X-ray diffraction (section 4.2) and Raman spectroscopy (section 4.3). Photoluminescence and cathodoluminescence (section 4.4) were used to quantify the optical impact and activation of transition metal impurities within ZnO. Section 4.5 introduces the determination of a color and its specification in numbers. Deep level transient spectroscopy (section 4.6) was used to get an better understanding of the deep centers which are involved in the green luminescence band.

\subsection{Electron microscopy}

\section{Scanning electron microscopy}

For micro structural analysis of nanowires after growth and ion implantation the Scanning Electron Microscope (SEM) Leo Supra35 ${ }^{\mathrm{TM}}$ from the manufacturer Carl Zeiss SMT AG was used [267]. A SEM is ideal to distinguish the morphology of nanowire samples like alignment, diameter and aspect ratio. The Leo Supra35 TM is equipped with Gemini column, which is composed of a Shottky-field emission cathode $(\mathrm{ZrO} / \mathrm{W})$, electron lenses and secondary electron ring detector (In-Lense detector). Furthermore a Everhart-Thornley secondary electron detector [268] and a quadrant back scattering detector is available. It is possible to record an image by data signal mixing from the In-Lense detector and from the secondary electron detector, where the signal ration is variable. The acceleration voltage and the mag- 
nification of this microscope is continuously variable from $0.1 \mathrm{kV}$ to $30 \mathrm{kV}$ and from 12x to 900000x (manufacturer's data), respectively. The manufacturer specifies a maximal resolution of $2.5 \mathrm{~nm}$ at an acceleration voltage of $1 \mathrm{kV}$.

\section{Transmission electron microscopy}

Transmission electron microscopy (TEM) or high resolution transmission electron microscopy (HR-TEM) provides an insight into the properties of the crystal lattice on atomic scale. TEM is used to analyze crystal orientations, ion implantation defects (like dislocations), as well as the orientation and size of secondary phases within the host matrix.

Nanowires were transfered onto a copper grid, which were coated with a thin amorphous carbon film, by an imprint method. Thereby the copper grid is weakly pressed onto a nanowire sample, resulting in an adequate number of nanowires on the copper grid. The preparation of a TEM specimen of a single crystal is more complicated and was done with an focus ion beam (FIB) system from FEI (Nova 600 Nanolab). FIB milling becomes in recent years a standard tool for the preparation of TEM specimen of metals, ceramics, semiconductors, biological and geological materials $[269 ; 270]$. The preparation process starts with the deposition of a platinum line by electron beam and ion beam assisted chemical vapor deposition to prevent damage and sputtering of the top portion of the specimen. Platinum is provided by an organometallic gas via thin needle close to sample surface and ion/electron beam. With a $\mathrm{Ga}^{+}$ion beam $8 \mu \mathrm{m}$ depth trenches were milled on both sides of the platinum line resulting in the future TEM specimen between both trenches, as visible in figure 4.1a. This fairly thick specimen is thinned and subsequently cut free by the Ga ion beam, leaving only one bridge between specimen and the surrounding material. By platinum deposition the specimen was fastened to an Omniprobe nanomanipulator and additionally the connection to the sample was removed by the focused ion beam. The specimen was than mounted on a TEM sample holder by platinum deposition and thereupon cutted from the manipulator. A final thinning process was applied to reduce the specimen thickness to a few nanometers. The final TEM specimen of an 16 at.\% $\mathrm{Ni}$ implanted $\mathrm{ZnO}$ single crystal is shown in figure $4.1 \mathrm{~b}$.

The lattice properties of TM implanted $\mathrm{ZnO}$ single crystals were characterized with the Philips CM 200-FEG-UT at the 4th Institute of Physics at the University of Göttingen. This TEM works with an acceleration voltage of $200 \mathrm{kV}$ and a condenser lens system to govern the electron beam onto the sample. Thereby the sample is located within the objective lens. Thereafter the electron beam is focused onto a 


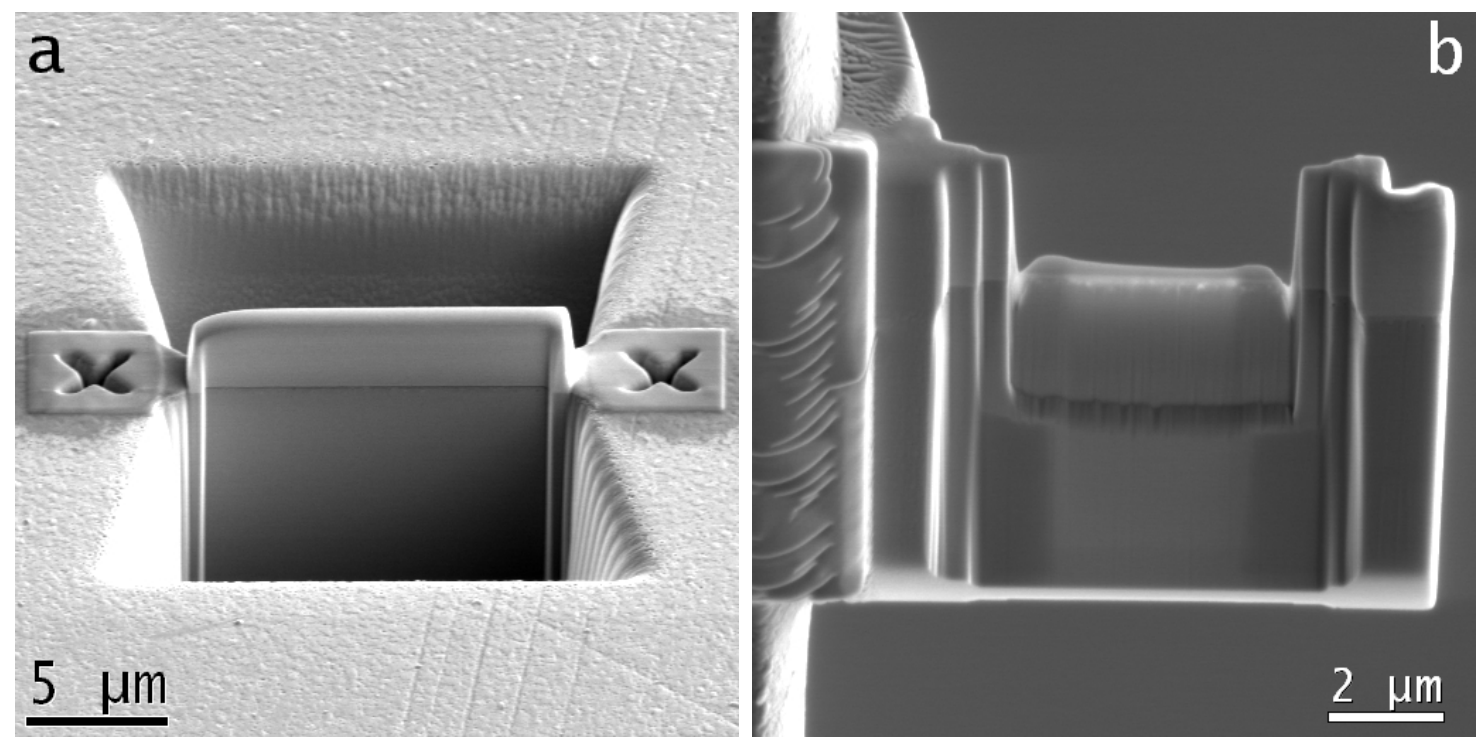

Figure 4.1: TEM specimen preparation by focused ion beam milling out of an 16 at.\% Ni implanted $\mathrm{ZnO}$ single crystal: (a) After deposition of a Pt metal line trenches on both sides of the metal line are milled. (b) shows the final TEM specimen after cutting and final thinning.

screen or a CCD camera. The Philips CM 200-FEG-UT has a maximum resolutions of $0.11 \mathrm{~nm}$. All TM implanted $\mathrm{ZnO}$ nanowires were characterized at the Chinese University of Hong Kong with a Philips CM120 and a FEI Tecnai 20 ST FEG, whereas the latter one is equipped with an electron energy loss spectrometer (EELS).

\section{Energy dispersive X-ray}

All electron microscopes are equipped with an energy dispersive X-ray spectrometer (EDS). The sample atoms were excited by the electron beam and the recombination energy from the excited state to the ground state of an atom is transformed into element characteristic X-ray radiation. EDS is a space-resolved X-ray fluorescence analysis [271], and provides a qualitative and quantitative elemental analysis. The lateral resolution is limited by the penetration depth of the electron beam in the material. The excited volume has a shape like a pear, whereas the size depends on the electron energy. Within pure zinc electron irradiation with an energy of $30 \mathrm{keV}$ results in an excited volume with a penetration depth of about $4 \mu \mathrm{m}$ and a diameter of about $1 \mu \mathrm{m}$ [272]. The reduced dimensions of a TEM specimen result in a smaller excited volume and therefore in a better space-resolution of some nanometer. The typical detection limit of an EDS system is about 1 at.\%. 


\subsection{X-ray diffraction}

X-ray diffraction (XRD) allows the study of the crystal quality and phase composition without destruction or modification. The periodical arrangement of crystal lattice planes with a lattice spacing of some Ångström results in the diffraction of $\mathrm{X}$-ray waves if there wavelength is less than the lattice spacing. The diffraction angle $\theta$ is a function of X-ray wavelength $\lambda$ and the lattice spacing $d_{h k l}$ of lattice planes with the Miller indices $h k l$ :

$$
2 d_{h k l} \sin \theta=n \lambda,
$$

whereas $n$ is a positive integer $[273 ; 274]$. A typical XRD spectrum shows several diffractions which are identified with the database values from [275].

The experimental XRD line profile is a convolution of the instrumental and physical curve which is furthermore a convolution of strain and crystallite size [274]. For the correction of the instrumental broadening a standard sample must be measured under instrumental conditions which are identical to the sample measurements. This standard sample must have the same chemical composition such as the sample material and the crystallite size must be large enough to eliminate all particle-size broadening. As we have no clear identification of the chemical composition of the secondary phases within $\mathrm{ZnO}$, the crystallite size was estimated with the Scherrer relation [276]:

$$
B=\frac{0.94 \cdot \lambda}{D_{B} \cos \theta}
$$

whereas $B$ is the full width at half maximum (FWHM), $\lambda$ is the X-ray wavelength and $D_{B}$ the crystallite size. For the calculation of the crystallite size the diffraction peak was fitted with the Gaussian function:

$$
f_{\text {Gauss }}=\frac{1}{\sigma \sqrt{2 \pi}} \exp \left(-\frac{1}{2}\left(\frac{x-\mu}{\sigma}\right)^{2}\right)
$$

whereas $\sigma>0$ is the standard deviation and $\mu$ the expected value. FWHM is than given by:

$$
F W H M=2 \sqrt{2 \ln 2} \sigma \approx 2.35 \cdot \sigma
$$

All XRD-spectra were measured with a D8 Discover from Bruker AXS [277] which works with a copper X-ray source at a wavelength of 1,540601 $\AA$. All spectra were taken in the unlocked $\theta-2 \theta$ mode from $25^{\circ}$ to $85^{\circ}$. Due to minor variances between the surface normal and the c-axis of the single crystals, all implanted single crystal samples were aligned via a fast $\theta-2 \theta$ scan and a subsequent rocking curve measure- 
ment. During the fast $\theta-2 \theta$ scan $2 \theta_{\text {fast }}$ and $\theta_{\text {fast }}$ of the (0002) diffraction of $\mathrm{ZnO}$ was determined, whereas $2 \theta_{\text {fast }}$ was used for the rocking curve measurement. Thereby the $2 \theta_{\text {fast }}$ angle between the sample and the detector is fixed and the intensity is measured as function of the incident beam angle $\theta$. The rocking curve gives the perfect $\theta_{\text {rocking }}$ angle and the discrepancy between $\theta_{\text {fast }}$ and $\theta_{\text {rocking }}$ is the adjustment of $\theta$ for the unlocked $\theta-2 \theta$ measurement.

\subsection{Raman spectroscopy}

Raman spectroscopy is a non-invasive research method for chemical analysis or solid state physics by inelastic scattering of monochromatic light within the studied sample [278-280]. It provides access to the lattice dynamics with the information about the chemical composition, orientation, or crystalline quality [281]. However, electronic and magnetic properties could be addressed, by Raman resonance effects and Raman scattering from magnons, respectively [281]. All this informations were obtained from frequency position and intensity, frequency width, and line shape.

The Raman shift is given by the frequency difference between the scattered light and the monochromatic excitation light due to the generation or annihilation of elementary excitations $[278 ; 279 ; 281]$. The excitation light transfers an electron onto a virtual electronic state or an excited electronic state, whereas the latter one is designated as resonant Raman scattering and shows an enhanced intensity if the number of such electronic states is large [278; 279]. During the recombination of this virtual electron state energy could be transfered via generation (Stokes Raman scattering) or annihilated (Anti-Stokes Raman scattering) of elementary excitation which results in positive or negative Raman shift (see figure 4.2) [279; 281], respectively. Below room temperature only few phonons are thermally excited, therefor the Stokes scattering process is dominant [281]. All presented Raman spectra show only the spectral part of Stokes Raman signals, whereas all spectra are recorded with a small offset to the Rayleight scattering peak at $0 \mathrm{~cm}^{-1}$ (see figure 4.2). During the inelastic scattering process energy from the incident photon $\hbar \omega_{i}$ is transfered to the sample by generation or vice versa by annihilation of elementary excitation, which is assumed to be phonons [281]. The energy $\hbar \Omega_{s}$ and wave vector $\vec{q}_{j}$ of the participating phonon are given by $[278 ; 279]$ :

$$
\begin{array}{rlll}
\text { Stokes process: } & \hbar \omega_{i}-\hbar \omega_{s}=\hbar \Omega_{s} & \text { and } & \vec{k}_{i}-\vec{k}_{s}=\vec{q}_{j} \\
\text { Anti-Stokes process: } & \hbar \omega_{s}-\hbar \omega_{i}=\hbar \Omega_{s} & \text { and } & \vec{k}_{s}-\vec{k}_{i}=\vec{q}_{j}
\end{array}
$$




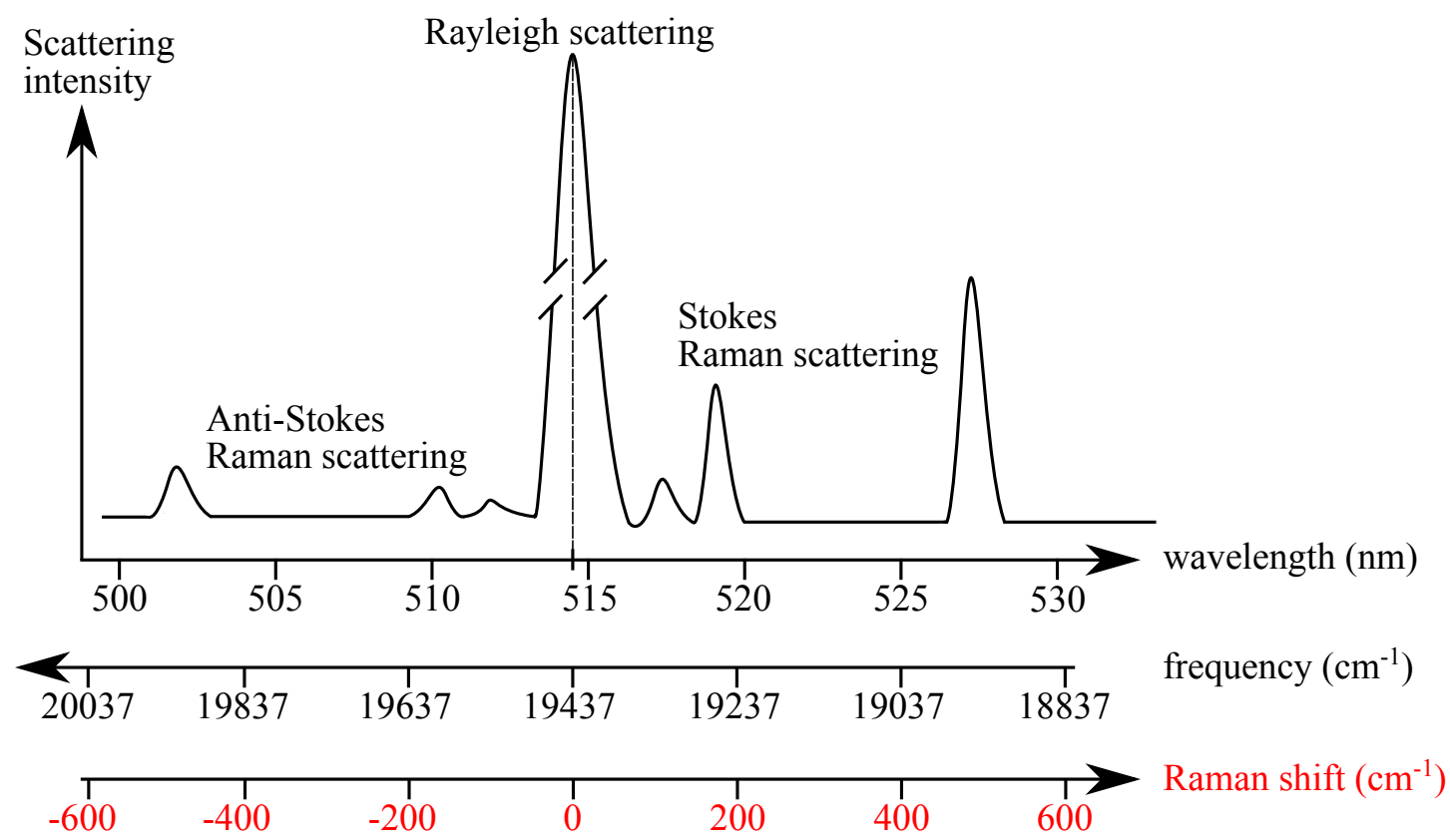

Figure 4.2: Schematic drawing of an Raman spectrum excited with an green laser source (514.5 $\mathrm{nm}$ ) representing the positive wavelength shift of Stokes Raman scattering, the negative shift of Anti-Stokes scattering, and Rayleight scattering (from [281]).

whereas $\hbar \omega_{s}$ is the energy of the scattered photon, $\vec{k}_{i}$ and $\vec{k}_{s}$ are the wave vectors of the incident and scattered light, respectively. Raman scattering involving elementary excitations are characterized by well-defined $(\Omega, q)$ pairs due to the conservation of energy and momentum [278; 279]. All Raman measurements within this thesis were applied in backscattering geometry, by what the momentum vectors (4.5) could be simplified into scalar functions [281]:

$$
\text { For Stokes process: } q_{j}=\frac{1}{c}\left(n\left(\omega_{i}\right) \omega_{i}-n\left(\omega_{s}\right) \omega_{s}\right) \text {, }
$$

whereas $c$ is the velocity of light and $n(\omega)$ is the index of refraction. Due to the much longer wavelength of incident $\lambda_{i}$ and scattered light $\lambda_{s}$ compared to the lattice constant $a_{0}$ and much smaller wave vectors $k_{i}, k_{s}$ and $q_{j}$ than the wave vector of the Brillouin zone boundary, the phonon wave vector is: $q_{j} \approx 0$ for first order Raman scattering. Therefore, one-phonon Raman scattering is restricted to radiationphonon interactions at the center of the Brillouin zone [278; 279], which is in contrast to multi-phonon scattering. In that case only the sum of the phonon wave vectors must be close to zero, for which reason also phonons from outside the Brillouin zone 


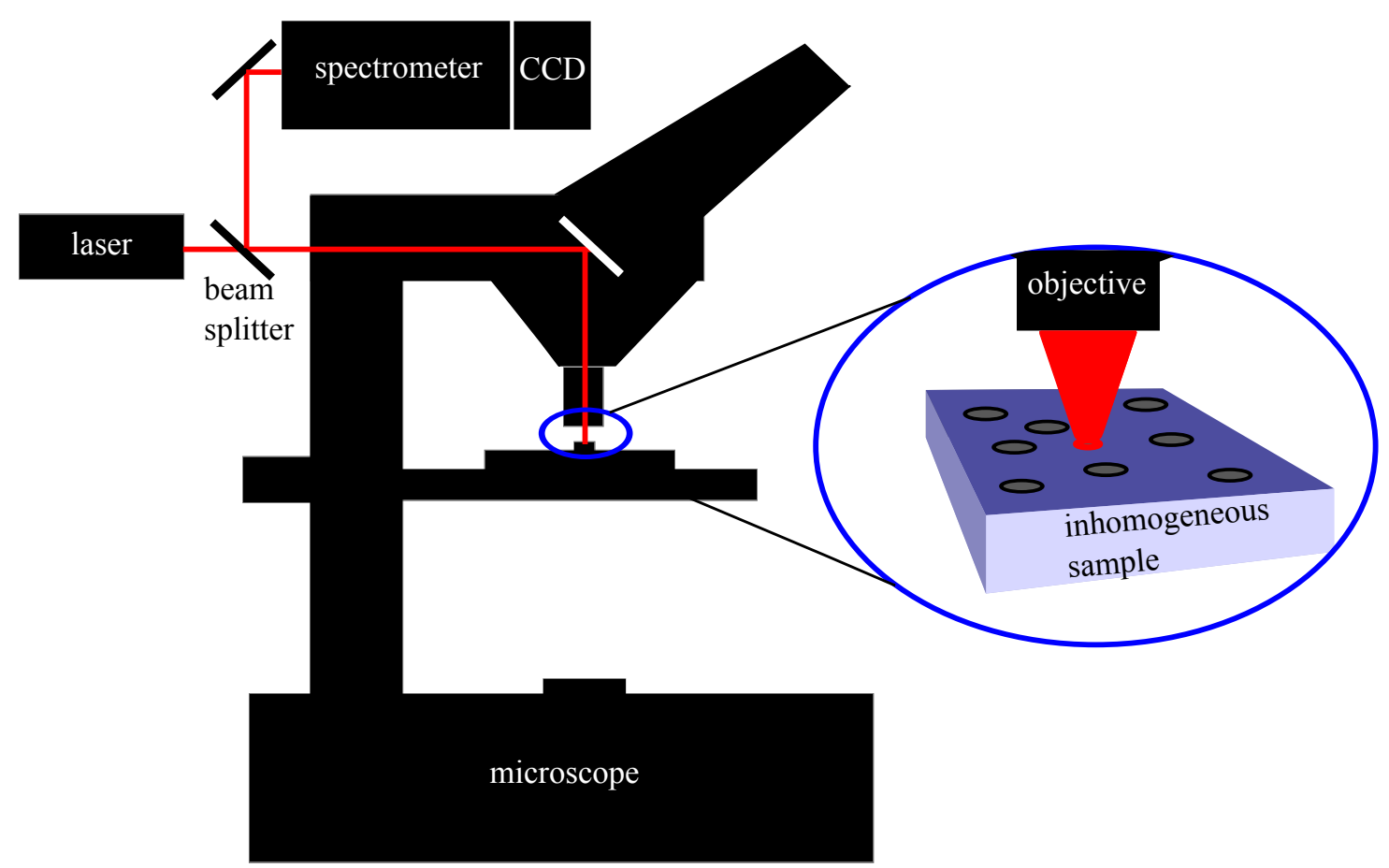

Figure 4.3: Schematic drawing of a micro-Raman setup: The excitation laser light is inserted through a beam splitter and focused on the sample by an objective. The backscattered light is collected by the objective and deflected by the beam splitter into the spectrometer (from [281]).

center can be involved. A detail description of the Raman method and theory is given in [278-281].

All Raman measurements were performed as micro-Raman or macro-Raman experiments in collaboration with the work group of Prof. Dr. J. Geurts at the University of Würzburg. An optical microscope was inserted into the setup for micro-Raman measurements (see figure 4.3) [281]. Micro-Raman scattering with an optical microscope gives the advantage for a lateral resolution with the opportunity to detect and record inhomogeneities on the sample surface [281]. The excitation laser light is coupled into the microscope (Leica DM LM on Renishaw Raman system RM 1000 and Olympus BHT on Dilor XY) via a beam splitter and focused onto the sample by an objective (Leica DM LM: 50x/Olympus BHT: 10x, 50x ULWD ${ }^{1}$, 80x ULWD, and 100x) [281]. As visible in figure 4.3, the objective lens collects the Raman scattered light from the sample which is then split from the excitation laser light by the beam

${ }^{1}$ Ultra Long Working Distance objective 
splitter and deflected into the spectrometer Renishaw RM $1000^{2}$ or Dilor XY $\mathrm{XY}^{3}$ [281]. Thereby, the Ramishaw setup is equipped with a camera for optical photography. For excitation several laser lines from an argon ion laser ${ }^{4}$ or a HeNe laser ${ }^{5}$ [281]. The Dilor XY sample holders for mirco-Raman and macro-Raman measurements are equipped with a liquid helium cryostat, whereas the macro-Raman setup could be utilized as magneto-cryostat with a maximum magnetic field of 6 Tesla [281].

\subsection{Photo- and cathodoluminescence}

Extrinsic and intrinsic defects within a semiconductor introduce donor or acceptor states within the band gap and could be characterized by photoluminescence (PL) and cathodoluminescence (CL) measurements. Thereby a semiconductor is excited with accerlerated electrons (CL) or high energetic photons (PL). Three different lasers were used for excitation: two HeCd laser, an InGaAs laser and a Nd:YAG laser. Both HeCd lasers are continuous wave (cw) lasers with a wavelength of $325 \mathrm{~nm}$ and a power output of $25 \mathrm{meV}$ (TOPAG HCL-30Ymc) and $50 \mathrm{meV}$ (KIMMON KOHA CO., LTD; IK3552R-G). The pulsed Nd:YAG laser (Quantel/Big Sky Laser Technologies Inc. - Ultra CFR) is equipped with a frequency doubler $(\lambda=532 \mathrm{~nm})$ and a frequency quadrupler $(\lambda=266 \mathrm{~nm})$. The Nd:YAG laser emits pulses at $266 \mathrm{~nm}$ with a length of $5 \mathrm{~ns}$ and a variable frequency from 1 to $20 \mathrm{~Hz}$. The pulse power is tunable within twenty power levels between $12 \mathrm{~kW} / \mathrm{cm}^{2}$ and $3 \mathrm{MW} / \mathrm{cm}^{2}$. The InGaAs laser is a frequency doubled diode laser emitting light at $535 \mathrm{~nm}$. All lasers were coupled into the vacuum chamber via two dielectric high performance mirrors onto the sample with an angle of $60^{\circ}$ to the sample normal (see figure 4.4). For cathodoluminescence measurements an electron gun was mounted on top of the vacuum chamber, where the acceleration voltage and the beam current could be varied between 0.5 to $5 \mathrm{keV}$ and 0.1 to $50 \mu \mathrm{A}$, respectively. All samples were mounted on a copper block with conductive silver, where the copper block was attached to helium cooling stage (CTI Cryogenics 22C/350C). Around the copper sample holder a cooling shield was attached to the cooling stage and is held at a temperature of about $12 \mathrm{~K}$ during the measurements. On the cooling stage a resistance heating element was installed. The temperature was controlled by a LakeShore 331 temperature controller. The temperature could be varied from $12 \mathrm{~K}$ to $300 \mathrm{~K}$ via a silicon

\footnotetext{
2 spectral resolution: $4 \mathrm{~cm}^{-1} /$ pixel

${ }^{3}$ spectral resolution: $1 \mathrm{~cm}^{-1} /$ pixel in low dispersion and $0.3 \mathrm{~cm}^{-1} /$ pixel in high dispersion mode

${ }^{4}$ Laser lines: $457.9 \mathrm{~nm}, 476.5 \mathrm{~nm}, 488 \mathrm{~nm}, 496.5 \mathrm{~nm}$, or $514.5 \mathrm{~nm}$

${ }^{5}$ Laser line: $632.8 \mathrm{~nm}$
} 


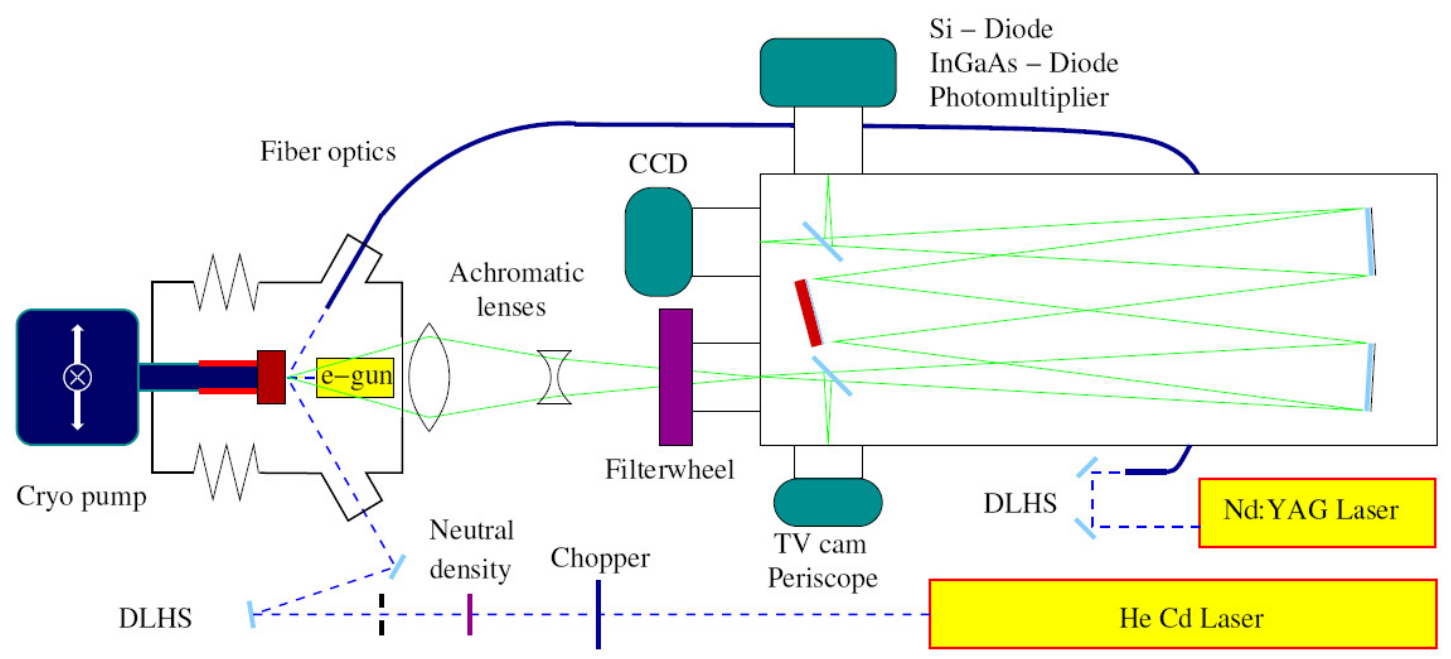

Figure 4.4: Schematic drawing of the photoluminescence (PL) and cathodoluminescence (CL) equipment: The sample is placed on a copper holder which is mounted on a cryo cooling stage. Excitation could be done with a HeCd laser, a Nd:YAG laser or an electron gun. The sample luminescence is directed by an achromatic lens system into the monochromator and measured with a CCD detector or a photomultiplier (taken from [227]).

temperature diode (DT 430) within the copper block. One or two achromatic lenses were used to focus the luminescence light onto the entrance slit of the monochromator. The monochromator is a computer controlled Czerny-Turner spectrograph (Jobin Yvon Horiba 1000M) with a focal length of one meter. The dispersion of the luminescence spectrum is created by exchangeble gratings within the monochromator. Three different gratings ${ }^{6}$ are available and the monochromator is equipped with two exit slits. A liquid nitrogen cooled CCD camera (Jobin-Yvon Horiba Spektrum One) with a resolution of $1024 \times 128$ pixel is attached on the axial exit slit and has a working range between $200 \mathrm{~nm}$ and $1100 \mathrm{~nm}$. A photomultiplier (Hamamatsu) is attached on the second exit slit, where a mirror deflects the luminescence light onto the photomultiplier. The photomultiplier is operating in the photocounting mode and has a working range between $190 \mathrm{~nm}$ and $750 \mathrm{~nm}$. The monochromator was calibrated with a mercury arc lamp (Oriel 6035) with closed entry slits. The spectra

\footnotetext{
${ }^{6}$ Grating 1: 1200 lines per $\mathrm{mm}(300 \mathrm{~nm})$, Grating 2: 600 lines per mm $(700 \mathrm{~nm})$, Grating 3: 100 lines per mm (450 nm).

The values in brackets refer to the blaze wavelength.
} 
were calibrated with the line at $\lambda=365,48 \mathrm{~nm}$ for high resolution measurements and the $\lambda=546,07 \mathrm{~nm}$ line for survey measurements [282].

\subsection{The measurement of color}

The standard of color measurement was defined by the Commission internationale de l'Éclairage (CIE) in 1931 which is known as the CIE 1931 or CIE XYZ color space. The human eye was taken as basis of this color standard, where the three types of cone cells in the retina of the eye are sensitive to short (blue), middle (green) or long (red) wavelengths. The human color vision is generated by varying amounts of these tristimulus values $X, Y$ and $Z$ which results in a three dimensional color space CIE XYZ. With the CIE primaries $\bar{x}, \bar{y}$ and $\bar{z}$ which are shown in figure $4.5 \mathrm{a}$, the tristimulus values $X, Y$ and $Z$ of the presented luminescence measurements were calculated by [283-285]:

$$
\begin{aligned}
& X=\int_{0}^{\infty} I(\lambda) \bar{x}(\lambda) d \lambda \\
& Y=\int_{0}^{\infty} I(\lambda) \bar{y}(\lambda) d \lambda \\
& Z=\int_{0}^{\infty} I(\lambda) \bar{z}(\lambda) d \lambda
\end{aligned}
$$

where $I(\lambda)$ is the intensity of the recorded photoluminescence spectrum. For color specification it is necessary to distinguish between chromaticity and luminosity. Luminosity defines the brightness of a color ranging from white to black. The ratios between the tristimulus values of white and gray are equal whereas the tristimulus values of gray have a smaller magnitude, so gray has a lower brightness than white. The quality of color is determined by the chromaticity which is a function of the tristimulus values $X, Y$ and $Z$ and is specified by the chromaticity coordinates [283-285]:

$$
\begin{aligned}
& x=\frac{X}{X+Y+Z} \\
& y=\frac{Y}{X+Y+Z} \\
& z=\frac{Z}{X+Y+Z}
\end{aligned}
$$

Taking into account that $x+y+z=1$, only two coordinates are necessary to specify the chromaticity of a material, here $x$ and $y$ [283-286]. The chromaticity values of our samples are plotted in the so called chromaticity diagram, shown in figure 4.5b. The solid black boundary is the locus of all pure monochromatic light sources. A real light emitter has a more or less broad spectral distribution and is located inside 

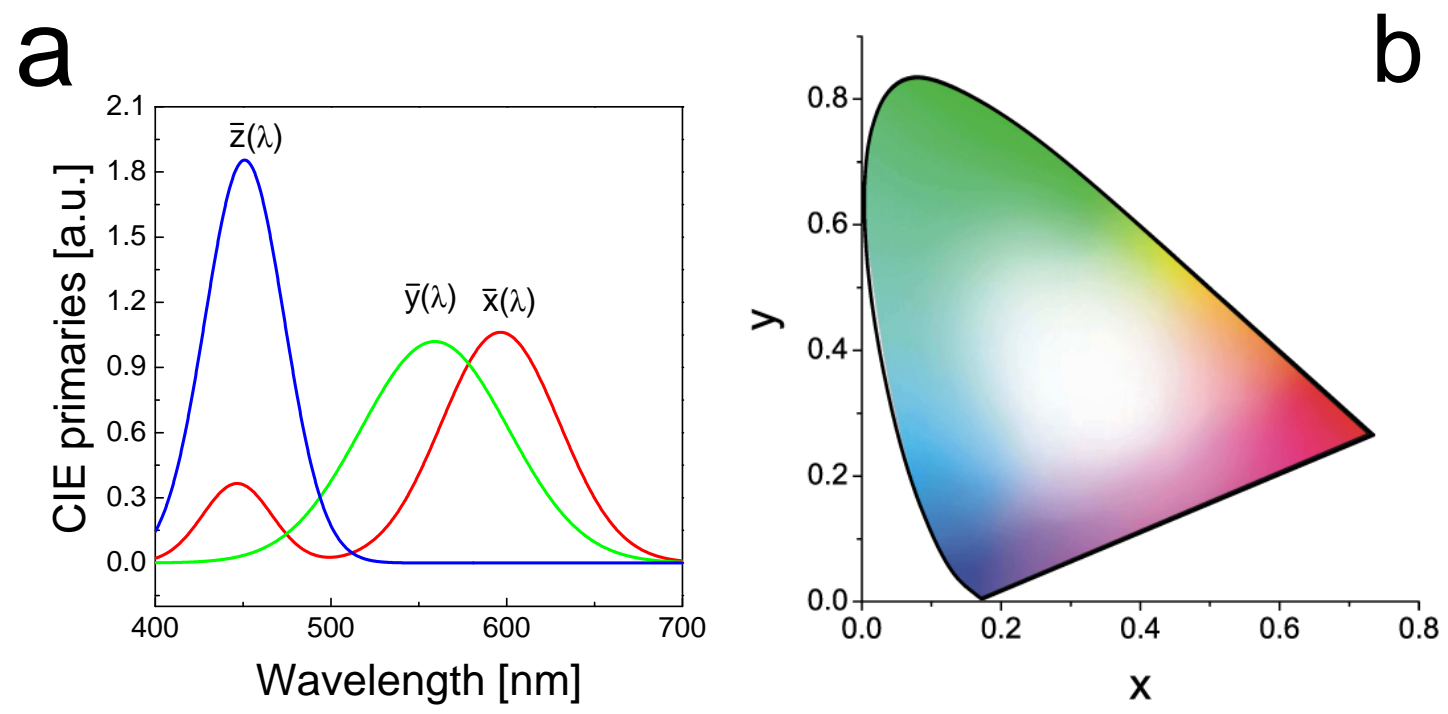

Figure 4.5: (a) shows the CIE primaries which represent the color vision of a human eye for red $[\bar{x}(\lambda)]$, green $[\bar{y}(\lambda)]$ and blue $[\bar{z}(\lambda)]$ (values are taken from [283]). (b) is the chromaticity diagram expressing colors in numbers.

or unto the spectral locus $[283 ; 284]$. The specific color regions blue, green, red and white are indicated in the diagram.

\subsection{Deep-level transient spectroscopy}

Luminescence experiments are only capable for the verification of radiative centers and their position within the band gap. Nonradiative centers could be detected by measuring the capacitance of a $\mathrm{p}-\mathrm{n}$ junction or a Schottky barrier by changing the charge state of the center. Deep-level transient spectroscopy (DLTS) is a high frequency ( $\mathrm{MHz}$ range) junction capacitance transient thermal scanning technique. A positive or negative peak on a flat base-line plotted as a function of temperature indicates the presence of each trap, whereas the heights are proportional to their respective trap concentration and the sign indicates whether it is a majority- or minority-carrier trap. It is possible to determine the thermal emission rate, activation energy, concentration profile, and the capture rate of each trap. A detailed description of the DLTS method and theory is given in $[287 ; 288]$ and references therein.

Three samples with an additional $0.2 \mu$ m thick $1 \%$ Al-doped $\mathrm{ZnO}$ bottom contact layer between the $1 \mu \mathrm{m}$ thick nominally undoped $\mathrm{ZnO}$ and the sapphire substrate have been grown by PLD under the same conditions as the implanted samples used 
for PL. After identical ion implantation and thermal treatment, circular Schottky contacts were fabricated by thermal evaporation of Pd [289]. We recorded the capacitance transients up to $300 \mathrm{~K}$ in steps of $1 \mathrm{~K}$ at different period widths $T_{w}$ and filling pulse times $t_{p}$ ranging between 5 and $500 \mathrm{~ms}$ using a FT 1030 DLTS system and a 'square-lock-in' correlation function with a largest detectable emission rate of $e_{i}=15000 \mathrm{~s}^{-1}$. All deposited films were n-type conducting with an apparent free charge carrier concentration increasing from $2 \cdot 10^{17} \mathrm{~cm}^{-3}$ in the surface near region to $8 \cdot 10^{17} \mathrm{~cm}^{-3}$ close to the $\mathrm{Al}$-doped $\mathrm{ZnO}$ layer, as determined by $\mathrm{CV}$-measurements (see results). This could be partially due to the diffusion of $\mathrm{Al}$ from the bottom contact layer into the undoped $\mathrm{ZnO}$ and to the partial compensation of intrinsic defects by implantation induced defects. DLTS measurements were made in collaboration with the work group of Prof. Dr. Grundmann at the University of Leipzig. 


\section{Chapter 5}

\section{Structural impact of transition metal implantation into $\mathrm{ZnO}$}

Ion implantation induces a high number of defects in the solid target material with different chemical and structural nature by transferring the ion energy into the target lattice $[265 ; 290]$. The impinging ion knocks out target atoms from their lattice sites, producing vacancies, interstitials, atoms on antisites, and dislocations. High dose ion implantation could even lead to amorphization of crystalline materials. After the ion implantation process, the properties of the target material are dominated by those defects. Therefore, a technique is required to reduce the defect concentration after ion implantation. Commonly the ion implantation defects are healed by annealing at elevated temperature. None the less, it was shown that massive ion implantation of transition metal could form secondary phases especially after annealing [15-18].

This chapter presents the impact of ion implantation of transition metals on the $\mathrm{ZnO}$ lattice as well as the annealing effect in air after the ion implantation. First of all the crystal structure of pure $\mathrm{ZnO}$ nanowires and single crystals is presented, which is continued with the annealing behavior of the transition metal implanted $\mathrm{ZnO}$ samples. The chapter is closed with the identification of secondary phases formed in high dose implanted $\mathrm{ZnO}$ single crystals.

\subsection{Crystal properties of pure $\mathrm{ZnO}$}

$\mathrm{ZnO}$ has a wurtzite lattice structure with a stacking sequence ..ABAB.. along the c-axis, which is illustrated in figure 5.1. Every stacking sequence is composed of two hexagonal plains, one is occupied with zinc atoms the other with oxygen atoms. Whereby, both plains are arranged in hexagonal closed package. The wurtzite lattice 


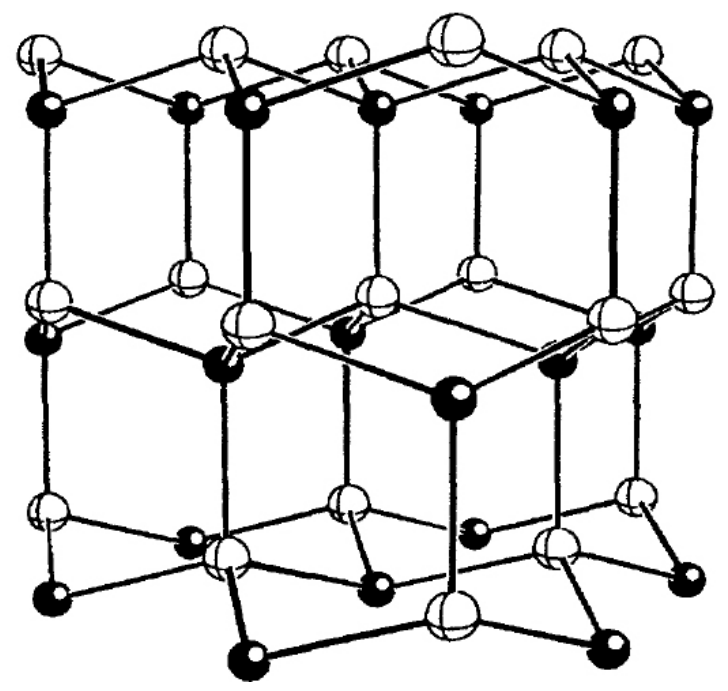

Figure 5.1: Wurtzite lattice structure of $\mathrm{ZnO}$, the black and white colored spheres represent zinc and oxygen atoms, respectively. (from [291]).

is an overlap of two hexagonal closed packages. The wurtzite $\mathrm{ZnO}$ phase has lattice constants of $a_{0}=3,29 \AA$ and $c_{0}=5,241 \AA$, with a ratio of $c_{0} / a_{0}=1,593$ [1]. All atoms within the $\mathrm{ZnO}$ lattice form ionic bonds between the $\mathrm{Zn}^{2+}$ and $\mathrm{O}^{2-}$ ions and every ion is bonded with four surrounding ions of the other species in tetrahedral symmetry. Besides the wurtzite $\mathrm{ZnO}$ phase a sphalerite $\mathrm{ZnO}$ phase exists with a lattice constant of 4,47 $\AA$, which can be fabricated under extreme conditions [292; 293].

The phase of the $\mathrm{ZnO}$ nanowires and the single crystals was identified by XRD and is shown in figure 5.2 for nanowires in (a) and for CrysTec single crystals in (b). The $\mathrm{ZnO}$ nanowires grow randomly distributed on the silicon substrates. Therefore, several diffraction reflexes are observed in XRD patterns of the nanowire samples, which are indicated in figure 5.2a and assigned to [237]: (10 $\overline{1} 0),(0002),(10 \overline{1} 1)$, $(10 \overline{1} 2),(11 \overline{2} 0)$ and (1013) lattice planes of wurtzite $\mathrm{ZnO}$ [275]. From this diffraction lines the lattice constants of the $\mathrm{ZnO}$ nanowires can be estimated to [237]: $a=3,25 \pm 0,01 \AA$ and $c=5,20 \pm 0,02 \AA$, which differ insignificantly from the reference values [1]. ZnO single crystals must be aligned before XRD measurements to improve the statistics (see section 4.2), a XRD diffraction pattern after alignment is shown in figure 5.2b. In contrast to $\mathrm{ZnO}$ nanowires, only the (0002) and (0004) diffraction can be observed in figure $5.2 \mathrm{~b}$, which are very sharp and show a good crystalline quality (note; the logarithmic scale).

A TEM and HR-TEM micrograph of an as-grown $\mathrm{ZnO}$ nanowire is shown in figure 5.3a and 5.3b [237], respectively. The survey in figure 5.3a suggests a single crystalline structure of an as-grown $\mathrm{ZnO}$ nanowire, neither extended defects nor stacking faults are observable. Figure $5.3 \mathrm{~b}$ is the HR-TEM micrograph of figure 

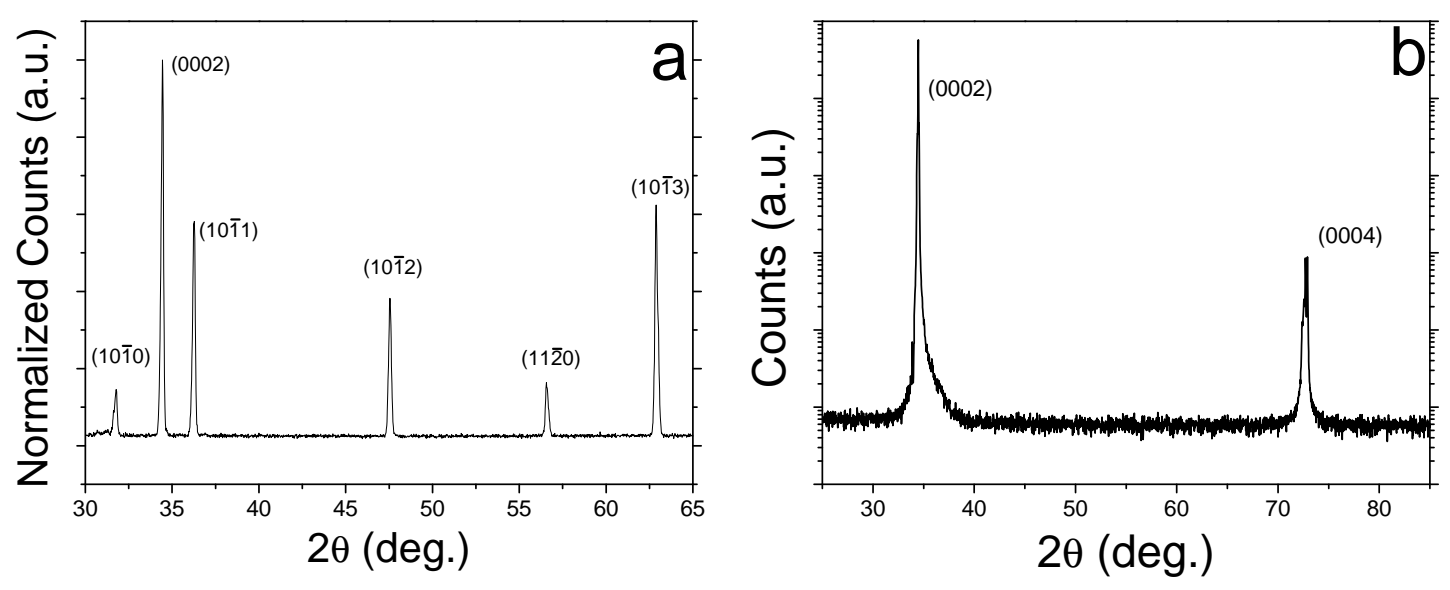

Figure 5.2: Due to the random distribution of $\mathrm{ZnO}$ nanowires on a sample, various XRD diffraction reflexes are indicated in (a) [237]: (1010), (0002), (1011),

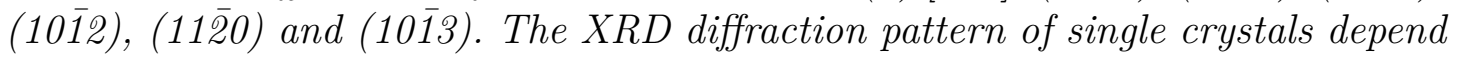
on the orientation of the crystal, (b) shows the XRD pattern of a single crystal with c-axis surface normal (plotted with logarithmic scale).

5.3a, the inset shows the fast Fourier transformation (FFT) of this micrograph. The HR-TEM micrograph shows unambiguously the single crystalline arragnement of the lattice with a high quality. The distance between the central point and the lightly displaced points above and below the central point are displaced by about $3.99 \mathrm{~nm}^{-1}$ in the FFT (inset in 5.3b), which equates to the lattice spacing of $0.25 \mathrm{~nm}$ and fits well to the distance between the (0002) lattice plains (0.2603 $\mathrm{nm}$ [294]). The reflexes, which are arranged in right angle to the (0002) reflexes in the FFT, have a distance of $7.24 \mathrm{~nm}^{-1}$ between each other. This value is consistent with an lattice spacing of $0.14 \mathrm{~nm}$ and matches the distance between the (2020) planes (0.1407 nm [294]). The corresponding lattice planes of the FFT reflexes are indicated in the FFT (inset in figure 5.3b). With respect to the results of the FFT image, the growth direction of the $\mathrm{ZnO}$ nanowires is the [0001] (c-axis) lattice direction. However, an review of the recent literature indicates the c-axis as the main growth direction of $\mathrm{ZnO}$ nanowires and nanorods [295-298]. The $\{10 \overline{1} 0\}$ lattice plane can be assigned as the side surfaces of $\mathrm{ZnO}$ nanowires from the FFT image and agree with literature [299; 300].

Figure 5.4 shows Raman spectra of one pure $\mathrm{ZnO}$ single crystal for different scattering configurations [281]. The Porto notation is used for the declaration of the scattering configurations $[281 ; 301]$, which presents the scattering configuration with:

$$
\vec{k}_{i}\left(\vec{\Pi}_{i} \vec{\Pi}_{s}\right) \vec{k}_{s}
$$



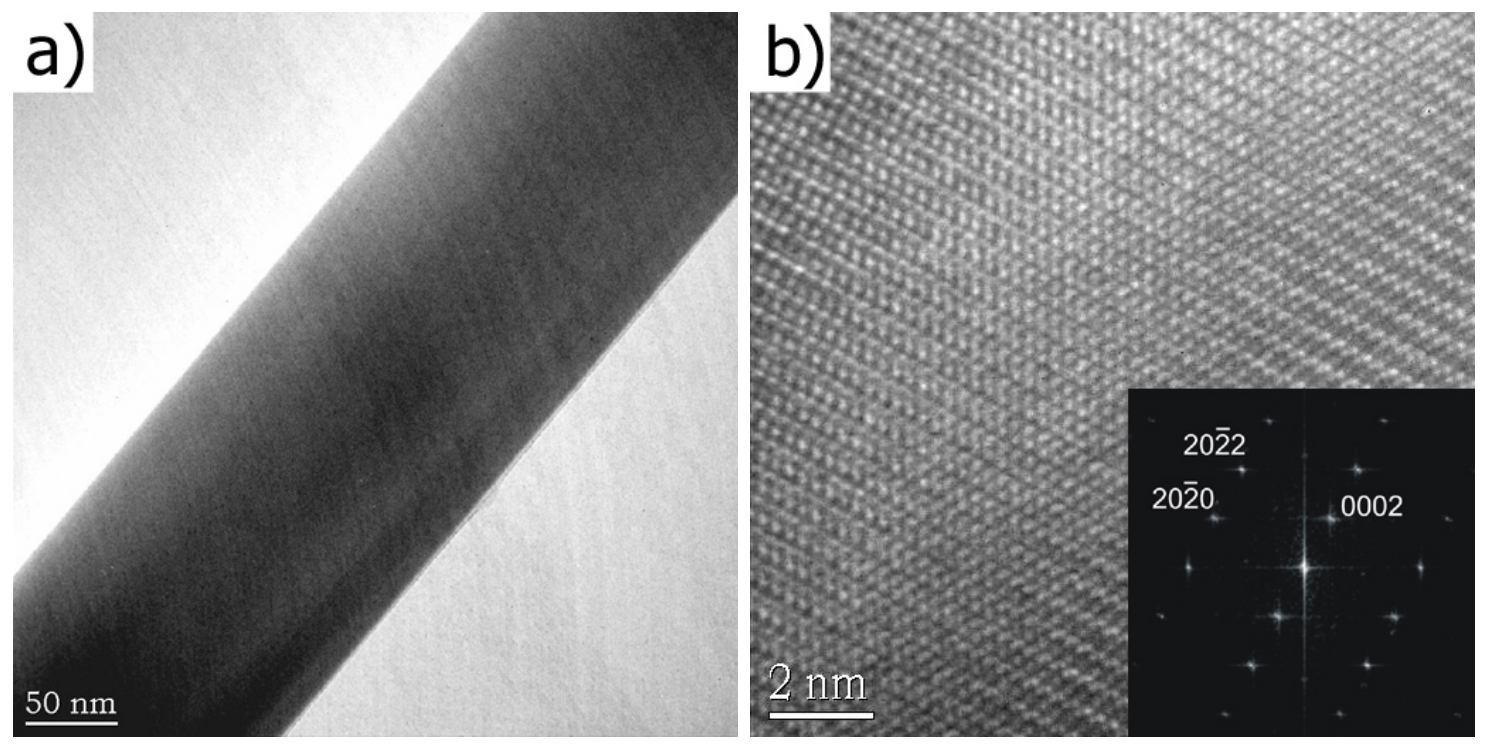

Figure 5.3: TEM micrograph of an as-grown ZnO nanowire with a diameter of about $165 \mathrm{~nm}$ shows no formation of stacking faults in (a). HR-TEM micrograph (b) of $Z n O$ nanowire in (a) reveal a uniform arrangement of the lattice without any dislocation (inset shows the FFT of (b)) [237].

where $\vec{k}_{i, s}$ is the propagation direction of light in the crystal and $\vec{\Pi}_{i, s}$ is the polarization of light. The index $i$ indicates the incident light and $s$ the scattered light. Thus, in $x(z z) \bar{x}$ scattering configuration the incident light is propagating in $x$ direction with polarization along $z$, the scattered light is propagating in $\bar{x}$ direction (backscattering direction) with polarization along $z$ [301]. However, the intensity of a lattice vibration mode in Raman spectroscopy depends in the classical model on [281]: $I_{R} \propto\left|\vec{\Pi}_{i} \Re \vec{\Pi}_{s}\right|^{2}$, where $\Re$ is the Raman tensor. $\Re$ is a symmetry tensor of rank two [281]:

$$
\Re=\left(\begin{array}{ccc}
\Re_{x x} & \Re_{x y} & \Re_{x z} \\
\Re_{y x} & \Re_{y y} & \Re_{y z} \\
\Re_{z x} & \Re_{z y} & \Re_{z z}
\end{array}\right)
$$

The Raman tensor $\Re$ represents the change of the Raman polarizability during the scattering process of the incident light and defines [281; 302], if a phonon mode is Raman active or not. A detailed description of the scattering theory as well as the Raman scattering tensor for different crystal symmetries is given in references [302; 303] and references therein.

All Raman spectra shown in figure 5.4 are recorded in backscattering configuration, whereby the $z(x x) \bar{z}$ configuration is commonly used for $\mathrm{ZnO}$ [281]. In almost 


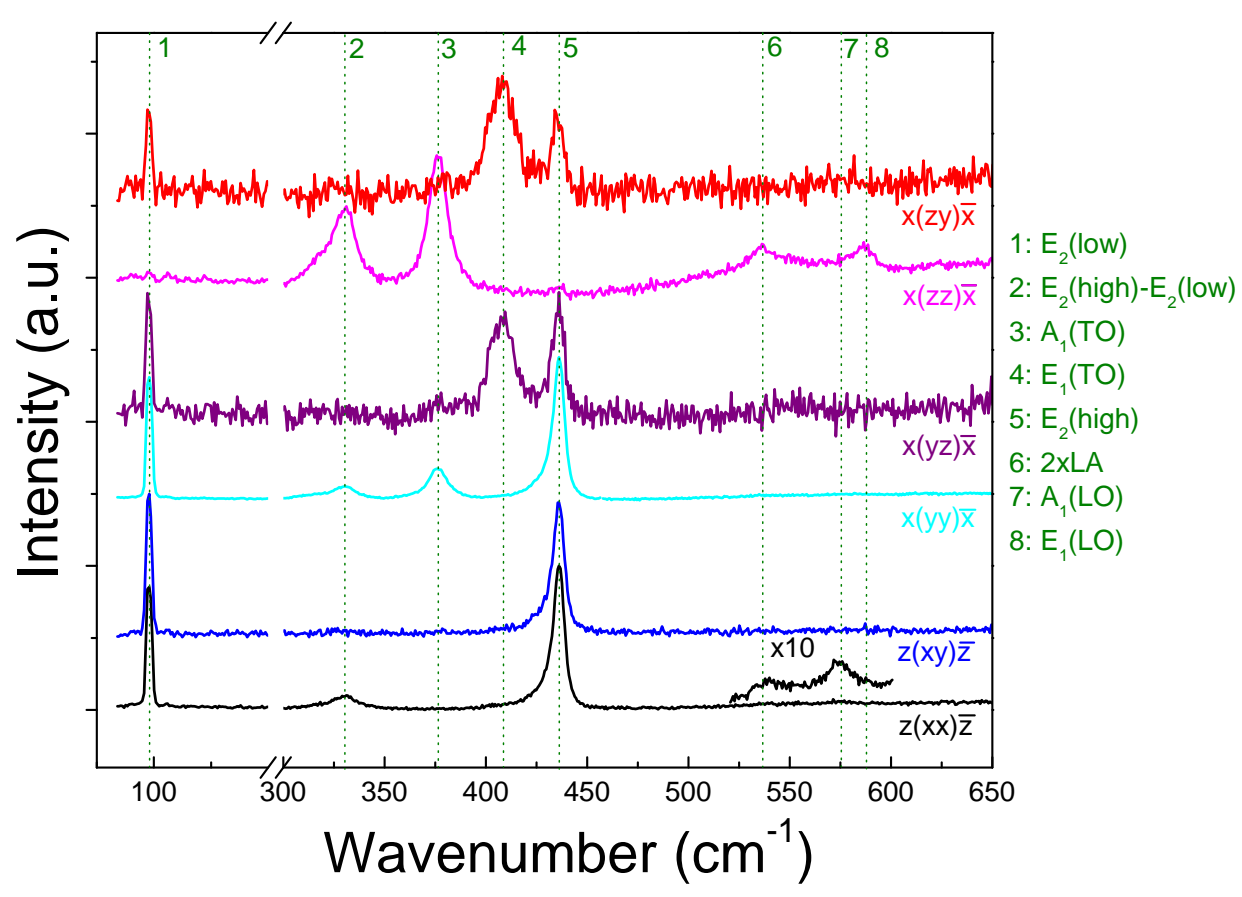

Figure 5.4: Raman spectra of pure $\mathrm{ZnO}$ recorded in different scattering configurations under excitation at $514.5 \mathrm{~nm}$ (all spectra taken at room temperature) [281]. The scattering configuration is given in Porto notation [301].

all Raman spectra in figure 5.4 two phonon modes at about $101 \mathrm{~cm}^{-1}$ and $437 \mathrm{~cm}^{-1}$ can be observed, which are assigned to the $E_{2}^{\text {low }}$ and $E_{2}^{\text {high }}$ phonon modes [303], respectively. Cusco et al. noticed that the $E_{2}^{\text {low }}$ mode involves mainly zinc motion and the $E_{2}^{\text {high }}$ mode mainly oxygen motion [303]. Both phonon modes dominated the Raman spectra in $z(x x) \bar{z}$ scattering configuration [303], whereby weak $A_{1}(L O)^{1}$ and $2 L A$ phonon modes can be observed (ten times enhanced interval in figure 5.4). At $333 \mathrm{~cm}^{-1}$ the $E_{2}^{\text {high }}-E_{2}^{\text {low }}$ difference mode is observed in several Raman scattering configurations, the assignment of this difference mode is supported by temperature dependent Raman measurements shown by Schumm and Cusco et al. [281; 303]. For the $z(x x) \bar{z}$ and $z(x y) \bar{z}$ only the up to now discussed phonon modes can be observed, but these modes can not be observed in $x(z z) \bar{x}$ configuration with exception of the $E_{2}^{\text {high }}-E_{2}^{\text {low }}$ difference mode. In $x(z z) \bar{x}$ and $x(y y) \bar{x}$ configuration an additional phonon mode at $378 \mathrm{~cm}^{-1}$ occurs, which is assigned to the $A_{1}(T O)$ mode [303]. TO modes are allowed in directions, where the incident and scattered light is perpendicular to the c-axis of $\mathrm{ZnO}$ [281; 303]. Therefore, the $E_{1}(T O)$ mode

\footnotetext{
${ }^{1}$ Typically, phonon modes are abbreviated with: $L O$ - longitudinal optical mode, $L A$ - longitudinal acoustical mode, $T O$ - transversal optical mode, and $T A$ - transversal acoustical mode.
} 
is observed additionally in the $x(y z) \bar{x}$ and $x(z y) \bar{x}$ configuration. Table 5.1 presents a list of phonon modes in $\mathrm{ZnO}$, which are identified by Raman spectroscopy and/or neutron scattering [88; 303-305].

Table 5.1: Phonon modes in $\mathrm{ZnO}$ including their energy, Brillouin zone point and scattering symmetry in Raman (adapted from [88; 303-305]).

\begin{tabular}{|c|c|c|c|c|}
\hline \multirow[t]{2}{*}{ Phonon mode } & \multicolumn{2}{|c|}{ Energy } & \multirow{2}{*}{$\begin{array}{c}\text { Brillouin zone } \\
\text { point/lines }\end{array}$} & \multirow{2}{*}{$\begin{array}{l}\text { Scattering } \\
\text { symmetry }\end{array}$} \\
\hline & $\left(\mathrm{cm}^{-1}\right)$ & $(\mathrm{meV})$ & & \\
\hline$T A, T O$ & 75 & 9.3 & $A$ & \\
\hline$E_{2}^{l o w}$ & 101 & 12.5 & $\Gamma$ & $\begin{array}{c}z(x x) \bar{z}, x(y y) \bar{x} \\
z(x y) \bar{z}\end{array}$ \\
\hline$B_{1}(L O)$ & 138 & 17.1 & $\Gamma$ & \\
\hline$L A, L O$ & 180 & 22.3 & $A$ & \\
\hline $2 T A, 2 E_{2}^{\text {low }}$ & 203 & 25.2 & $L, M, H ; \Gamma$ & \\
\hline$T O, L O$ & 243 & 30.1 & $\Gamma$ & \\
\hline$B_{1}^{\text {high }}-B_{1}^{\text {low }}$ & 284 & 35.2 & $\Gamma$ & \\
\hline$E_{2}^{\text {high }}-E_{2}^{\text {low }}$ & 333 & 41.3 & $\Gamma$ & \\
\hline$A_{1}(T O)$ & 378 & 46.9 & $\Gamma$ & $x(y y) \bar{x}$ \\
\hline$E_{1}(T O)$ & 410 & 50.8 & $\Gamma$ & $x(z y) \bar{x}, x(z y) y$ \\
\hline TO & 425 & 52.7 & $A$ & \\
\hline$E_{2}^{h i g h}$ & 437 & 54.2 & $\Gamma$ & $\begin{array}{c}z(x x) \bar{z}, x(y y) \bar{x} \\
z(x y) \bar{z}\end{array}$ \\
\hline $2 L A, L O$ & 489 & 60.6 & $M-K ; \Gamma$ & \\
\hline$L O, 2 B_{1}^{\text {low }}, 2 L A$ & 540 & 67 & $\Gamma ; L, M, H$ & \\
\hline$A_{1}(L O)$ & 574 & 71.2 & $\Gamma$ & $z(x x) \bar{z}$ \\
\hline$E_{1}(L O)$ & 590 & 73.2 & $\Gamma$ & $x(z y) \bar{y}$ \\
\hline$T A+T O$ & 618 & 76.6 & $H, M$ & \\
\hline$T A+L O$ & 657 & 81.5 & $L, H$ & \\
\hline$T A+L O$ & 666 & 82.6 & $M$ & \\
\hline$L A+T O$ & 700 & 86.8 & $M$ & \\
\hline$L A+T O$ & 723 & 89.8 & $L-M$ & \\
\hline$L A+T O$ & 745 & 92.4 & $L-M$ & \\
\hline$L A+T O$ & 773 & 95.9 & $M, K$ & \\
\hline$L A+L O$ & 812 & 100.7 & $L, M$ & \\
\hline
\end{tabular}




\subsection{Situation after ion implantation}

Figure 5.5 shows Raman spectra after ion implantation of $\mathrm{Ar}(\mathrm{a})$, Fe (b) and $\mathrm{Mn}$ (c) in $\mathrm{ZnO}$ single crystals [19], all spectra were recorded before annealing in air. Therefore, the Raman spectra show the disorder produced by the ion implantation process. All Raman spectra in figure 5.5 are recorded without polarization selection for the incident and scattered light to be sensitive for diagonal as well as off-diagonal Raman tensor $\Re_{i j}$ components. This corresponds to the detection of contributions of the $z(x x) \bar{z}$ and $z(x y) \bar{z}$ Raman scattering configuration [281]. In figure 5.5a the Raman spectrum of a pure $\mathrm{ZnO}$ single crystal is compared with Raman spectra after Ar irradiation with ion fluences of $6.3 \cdot 10^{16} \mathrm{~cm}^{-2}$ and $12.6 \cdot 10^{16} \mathrm{~cm}^{-2}$. These ion fluences given in figure 5.5a correspond to theoretical Ar concentrations of about 4 at. $\%$ and 8 at. $\%{ }^{2}$ respectively. The $E_{2}^{\text {high }}$ phonon mode is dominating all Raman spectra after Ar ion implantation. For low Ar ion fluences minor changes can be observed in figure $5.5 \mathrm{a}$ around the $A_{1}(L O)$ phonon mode between $500 \mathrm{~cm}^{-1}$ and $600 \mathrm{~cm}^{-1}$. The inset in figure 5.5a shows the interval around the $A_{1}(L O)$ phonon mode in more detail for Ar ion fluences from $1.6 \cdot 10^{16} \mathrm{~cm}^{-2}$ up to $6.3 \cdot 10^{16} \mathrm{~cm}^{-2}$. Only very small intensity increases can be observed with increasing Ar fluence. However, the sample implanted with the highest Ar fluence shows a drastic increase as well as a broadening of this band around the $A_{1}(L O)$ phonon mode. In addition the second-order signal strenght ( $2 x L O$ region) increases, too. This band around the $A_{1}(L O)$ phonon mode has a maximal intensity of about $20 \%$ of the $E_{2}^{\text {high }}$ phonon mode after ion implantation of Ar with a fluences of $12.6 \cdot 10^{16} \mathrm{~cm}^{-2}$.

Figures 5.5b and 5.5c show the Raman spectra of Fe and $\mathrm{Mn}$ implanted ZnO single crystals before annealing [19], for comparison the Raman spectra of pure as well as of Ar implanted $\mathrm{ZnO}$ from figure 5.5a are added in figure 5.5b. The $E_{2}^{\text {high }}$ phonon mode is clearly visible in all Raman spectra of Fe implanted ZnO in figure 5.5b. All Raman spectra of Fe implanted samples are dominated by the broad band around the $A_{1}(L O)$ phonon mode and show an increasing intensity of this band with increasing Fe concentration. On the other hand, a red shift of the band can be observed in all transition metal implanted samples. The Raman spectra for $\mathrm{Ni}$ and $\mathrm{Co}$ implanted $\mathrm{ZnO}$ single crystals are not shown at this point, but the samples implanted with $\mathrm{Ni}$ and Co show the same behavior as the Fe implanted $\mathrm{ZnO}$. However, a wide difference is not expected due to the similar mass of all the implanted ions. But figure $5.5 \mathrm{c}$ reveals a much more stronger vibration band

\footnotetext{
${ }^{2}$ I would like to remind the reader, that the Ar implantation fluences and energies are chosen with respect to $\mathrm{Fe}$ implantation into $\mathrm{ZnO}$.
} 

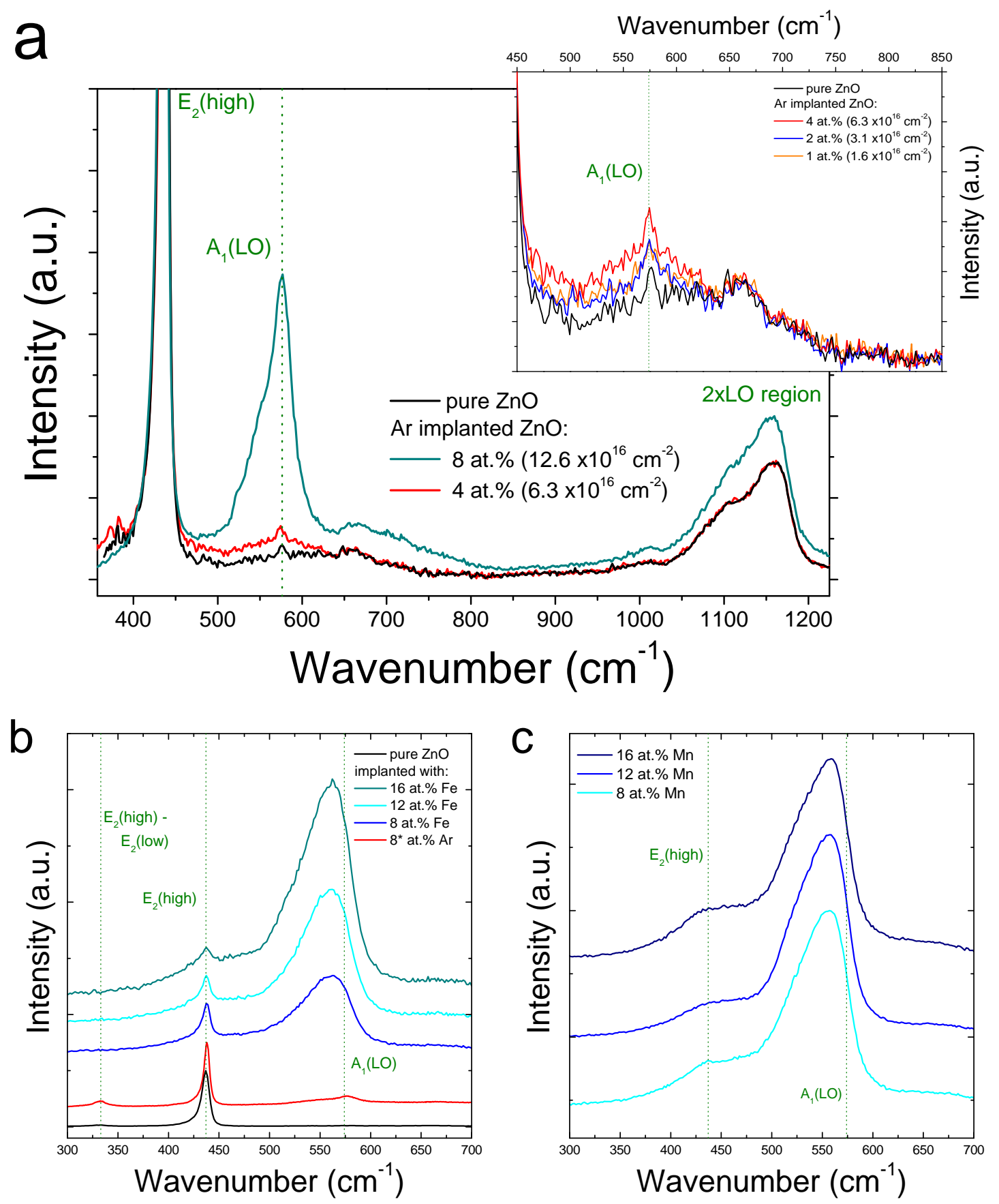

Figure 5.5: Raman measurements after ion implantation of $\mathrm{Ar}$ in (a), Fe in (b) and $M n$ in (c) show an increases of the $A_{1}(L O)$ phonon mode compared to the $E_{2}^{\text {high }}$ phonon mode [19]. The inset in (a) shows the low damage for low Ar ion fluences. All spectra are recorded under excitation at $514.5 \mathrm{~nm}$ at room temperature. 
around the $A_{1}(L O)$ phonon mode than all other transition metal implanted ZnO samples. The $E_{2}^{\text {high }}$ phonon mode could only be observed as a weak shoulder at about $437 \mathrm{~cm}^{-2}$. This observed difference between the Mn implanted samples and all other transition metal implanted samples can not be a result of the ion mass. Jin et al. had shown by absorption measurements that in ZnO:TM alloys defined absorption bands exists [306], which can be assigned to intra- $d$-shell transitions or to charge transfer processes of the incorporated transition metal ion (see discussion in section 6.4 and references therein). The Mn alloyed $\mathrm{ZnO}$ samples of Jin et al. reveal a broad absorption band below the band gap absorption edge, though, this band is assigned to a charge transfer process of the $\mathrm{Mn}^{2+}$ ion in $\mathrm{ZnO}$ or to intra- $d$-shell (see discussion in 6.4 .3 and references $[158 ; 307 ; 308])$. This band leads to a higher absorption of the excitation laser light (wavelength $514.5 \mathrm{~nm}$ ) in all $\mathrm{Mn}$ implanted samples than in all other transition metal implanted samples. Therefore, the transparency of the Mn implanted $\mathrm{ZnO}$ is strongly reduced, resulting in the excitation of only the implanted layer, which is commonly not the case. The sensitivity depth of the micro-Raman setup is between $5 \mu \mathrm{m}$ and $10 \mu \mathrm{m}$ [281], which is larger than the implantation depth. Typically, the Raman signal contains signals from the implanted layer as well as the intact single crystal below.

The additional band around the $A_{1}(L O)$ mode was observed after ion implantation for several different elements [309-313]. The broadening of the band around the $A_{1}(L O)$ phonon mode for the highest Ar implanted sample can be attributed to scattering contributions of the $A_{1}(L O)$ phonon branch outside the Brillouin zone center [281]. The ion implantation induces disorder, which reduces the crystal symmetry and gives rise to this phonon branch. The red-shift of this band in transition metal implanted samples is in accordance with the dispersion of the $A_{1}(L O)$ phonon branch near the $\Gamma$-point of the Brillouin zone [281]. Additionally it was shown that the $A_{1}(L O)$ phonon branch is affected by Raman resonance effects and is attributed to the strong resonances of $L O$ phonon modes [281; 313]. However, these resonances are dominated by Fröhlich scattering $[281 ; 313]$ and can be induced by impurities or defects. Commonly this $A_{1}(L O)$ phonon band is assigned to oxygen vacancy $[314 ; 315]$, zinc interstitials $[316 ; 317]$, or a defect complex containing both defect types [316]. Both defect types are generated during ion implantation, and are therefore a sign for the generated disorder.

The comparison between the Raman spectra of the 8 at.\% Ar implanted and the 8 at.\% Fe implanted sample in $5.5 \mathrm{~b}$ reveals a huge discrepancy of the main two Raman signals. It is necessary to notice that for both samples the same ion energies as well as the same ion fluences were used. The $E_{2}^{\text {high }}$ phonon mode dominates the 
Raman spectrum of the Ar implanted sample, but is of minor importance in the Raman spectrum of the Fe implanted sample. The $A_{1}(L O)$ phonon band shows a contrary behavior, and is dominant in the Raman spectrum of the Fe implanted sample. At first blance one may think that the observable differences between both Raman spectra are induced due to the mass difference between Ar and Fe. However, Kucheyev et al. had shown that the variation of collision cascade density by increasing ion mass has a negligible effect on the damage buildup behavior in $\mathrm{ZnO}$ [318; 319]. The authors had observed amorphization of ZnO by Si-implantation above an ion fluence of $4 \cdot 10^{16} \mathrm{~cm}^{-2}$ at $60 \mathrm{keV}$ [318], but only a strong damage for Auimplantation with $300 \mathrm{keV}$ and an ion fluence of $4 \cdot 10^{16} \mathrm{~cm}^{-2}$. The experiment of Kucheyev et al. indicates a more complicated process of defect formation during ion implantation. It is suggested that the ion implantation does not only produce lattice interstitials/vacancies but also impurity defects of the implanted species, which depend on their chemical effects [318]. The damange buildup during ion implantation is enhanced by chemical effects, if [320]: (I) an implanted impurity atom is trapped by an ion-beam-generated migrating point defect, (II) a secondary phase is formed, and/or (III) the irradiation-induced defects have an enhanced stability with a high concentration of the implanted species. Argon is chemical inert; thus, it can not be bonded in the crystal lattice. A major fraction should diffuse out of the crystal during the ion implantation, and at latest after annealing no Ar atoms should be inside the $\mathrm{ZnO}$ crystal lattice. On the other hand, all transition metal elements remain in the crystal, and capture a zinc lattice or produce defect complexes [83; 84]. As a result, the defect density in the Fe implanted sample should be higher than in the Ar implanted sample, giving rise to the $A_{1}(L O)$ phonon band.

\subsection{Annealing of defects}

The ion implantation damage dominates all physical properties of an as-implanted sample. The samples were annealed in air or oxygen ambient for reduction of ion implantation damage. Figure 5.6 summarizes the annealing effect for different annealing stages of $\mathrm{Ar}$ (a) and $\mathrm{Fe}$ (b) implanted $\mathrm{ZnO}$ single crystals as well as the situation after annealing at $700{ }^{\circ} \mathrm{C}$ for $\mathrm{Ni}$ implanted $\mathrm{ZnO}$ single crystals with different $\mathrm{Ni}$ concentrations. In figure 5.6a the Raman spectra of the as-implanted status of the $12.6 \cdot 10^{16} \mathrm{~cm}^{-2} \mathrm{Ar}$ implanted $\mathrm{ZnO}$ samples is compared to different annealing temperature stages. Annealing at $100{ }^{\circ} \mathrm{C}$ shows no major change to the as-implanted status, but further increase of the annealing temperature to $300{ }^{\circ} \mathrm{C}$ reveals a decrease 

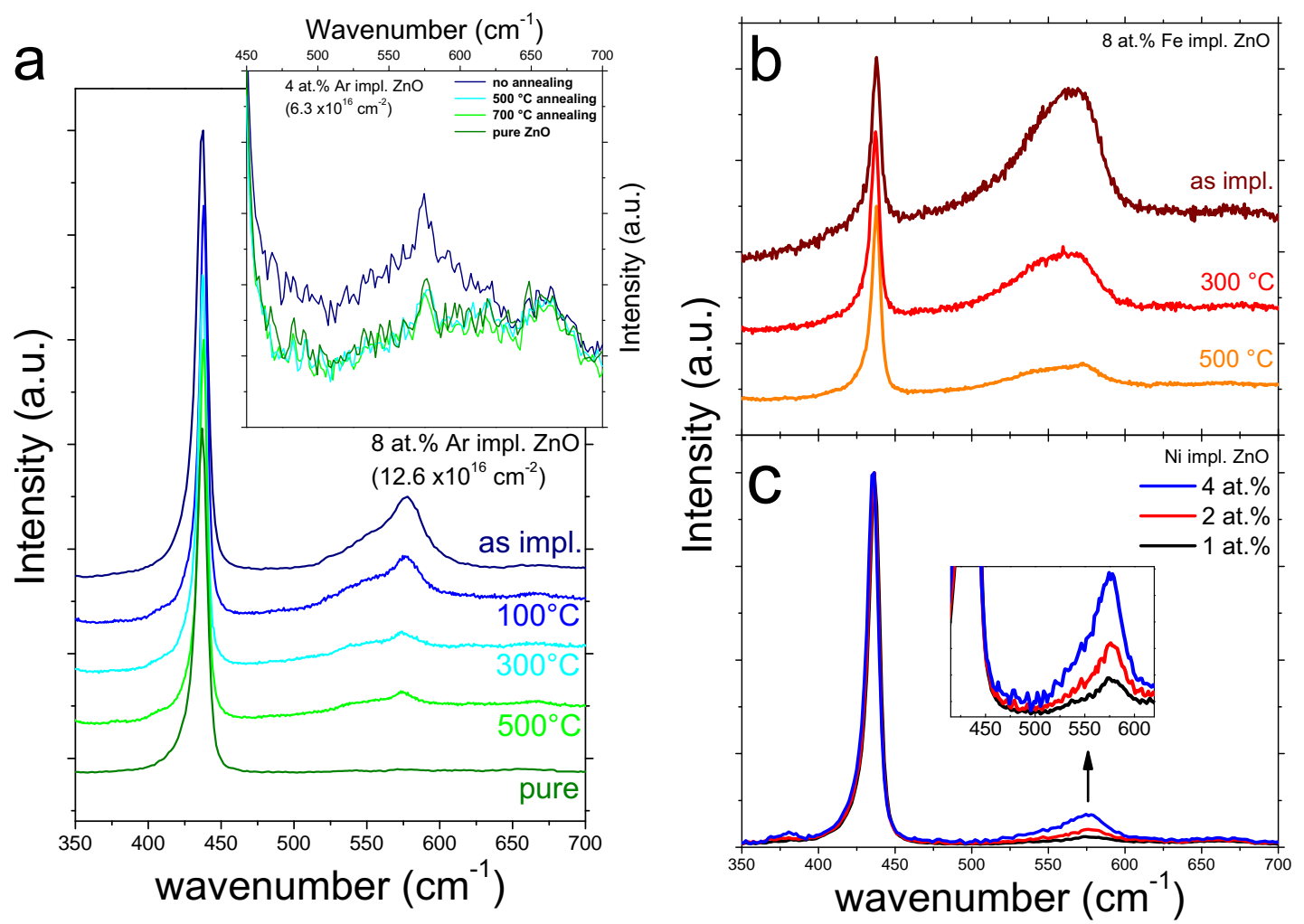

Figure 5.6: The annealing of $A r(a), F e(b)$ and $N i(c)$ implanted $Z n O$ single crystal reveals an decreasing $A_{1}(L O)$ phonon band in all Raman spectra (recorded at room temperature under excitation at $514.5 \mathrm{~nm}$ ) [19]. (c) illustrates the situation after annealing at $700{ }^{\circ} \mathrm{C}$ for $\mathrm{Ni}$ implanted $\mathrm{ZnO}$.

of the $A_{1}(L O)$ phonon band and the healing of the major part of ion implantation damage. A low intense $A_{1}(L O)$ phonon side band can be observed in figure $5.6 \mathrm{a}$ after annealing in air at $500{ }^{\circ} \mathrm{C}$. However, the inset in figure 5.6 a reveals the annealing process for a sample, which was implanted with a lower Ar ion fluence. After annealing at $500{ }^{\circ} \mathrm{C}$ no difference can be observed between the Ar implanted sample and a pure $\mathrm{ZnO}$ single crystal. These temperature is far below the melting point of $\mathrm{ZnO}\left(\sim 1975^{\circ} \mathrm{C}\right)$, typically annealing temperatures of $\sim 2 / 3$ of the melting point (in $\mathrm{K}$ ) are required for the healing of extended defects in semiconductors [321]. On the other hand, the annealing temperature depends on the character of the defect, especially on the chemical nature. Yoshiie et al. had reported that dislocations involving oxygen vacancies are removed by annealing at $300{ }^{\circ} \mathrm{C}$ [322]. The $\mathrm{Ar}$ ion implantation into $\mathrm{ZnO}$ results in the formation of vacancies and interstitials of both atomic species, which should recombine during annealing. The annealing in oxygen rich ambients (air or oxygen) was chosen to avoid the out-diffusion of oxygen during 
annealing as well as to restore the loss of oxygen during high dose ion implantation [318; 323]. It should be noticed at this point, that the luminescence measurements of Ar implanted and air/oxygen annealed $\mathrm{ZnO}$ samples show deep luminescence bands, which will be attributed to interstitial oxygen (see discussion of deep luminescence bands in section 6.3). Based on the assignment of the deep luminescence band, annealing in air/oxygen of $\mathrm{Ar}$ implanted $\mathrm{ZnO}$ leads to the incorporation of interstitial oxygen by in-diffusion of oxygen.

It is know that air annealing of transition metal alloyed $\mathrm{ZnO}$ favors the formation of secondary oxide phases [324]. However, the formation of secondary phases was also observed for vacuum annealed samples for low fluences [14-17]. The annealing behavior for transition metal implanted $\mathrm{ZnO}$ shows the same development (such as the 8 at.\% Fe implanted $\mathrm{ZnO}$ in figure 5.6b) as observed for Ar implanted $\mathrm{ZnO}$ in figure 5.6a. The annealing effect starts at $300{ }^{\circ} \mathrm{C}$ and annealing at higher temperatures leads to further reduction of the ion implantation damage (see figure 5.6). However, figure $5.6 \mathrm{c}$ shows, that after annealing at $700{ }^{\circ} \mathrm{C}$ the relative intensity of the $A_{1}(L O)$ phonon band increases with increasing transiton metal concentration, and therefore with increasing fluence. This observation would support the assignment of interstitial zinc to the $A_{1}(L O)$ phonon band [316; 317], because the implanted transition metals occupies a zinc lattice site, which was confirmed by emission channeling experiments $[83 ; 84]$. Therefore, a higher concentration of interstitial zinc is produced by the ion implantation of transition metals.

The emission channeling experiments of Rita el al. and Wahl et al. show that the perfect annealing temperature in vacuum is $800{ }^{\circ} \mathrm{C}$ for $\mathrm{Fe}$ and $600{ }^{\circ} \mathrm{C}$ for $\mathrm{Cu}$ $[83 ; 84]$. For higher annealing temperatures, the fraction of $\mathrm{Fe}$ and $\mathrm{Cu}$ on zinc lattice sites decreases due to diffusion and/or interaction with near surface defects [83; 84]. The enhanced diffusion as well as the interaction with defects would support the formation of secondary phases at higher annealing temperatures. However, the experiments were made with $\mathrm{Cu}$ and $\mathrm{Fe}$ concentrations of parts per million which is several orders of magnitude lower than transition metal concentrations used in this thesis. Therefore, the formation of secondary phases during annealing is more likely. All Raman and X-ray diffraction measurements of transition metal implanted ZnO with concentrations below 8 at.\% show no additional signal after annealing, which could be assigned to secondary phases (see for example figure 5.13 on page 82). However, both techniques are limited for the detection of secondary phases. Small secondary phase clusters with low volume ratio would show extreme low intense and broad diffraction peaks under high intensive X-ray diffraction measurements such as synchrotron XRD measurements. Therefore, such small phases are not de- 


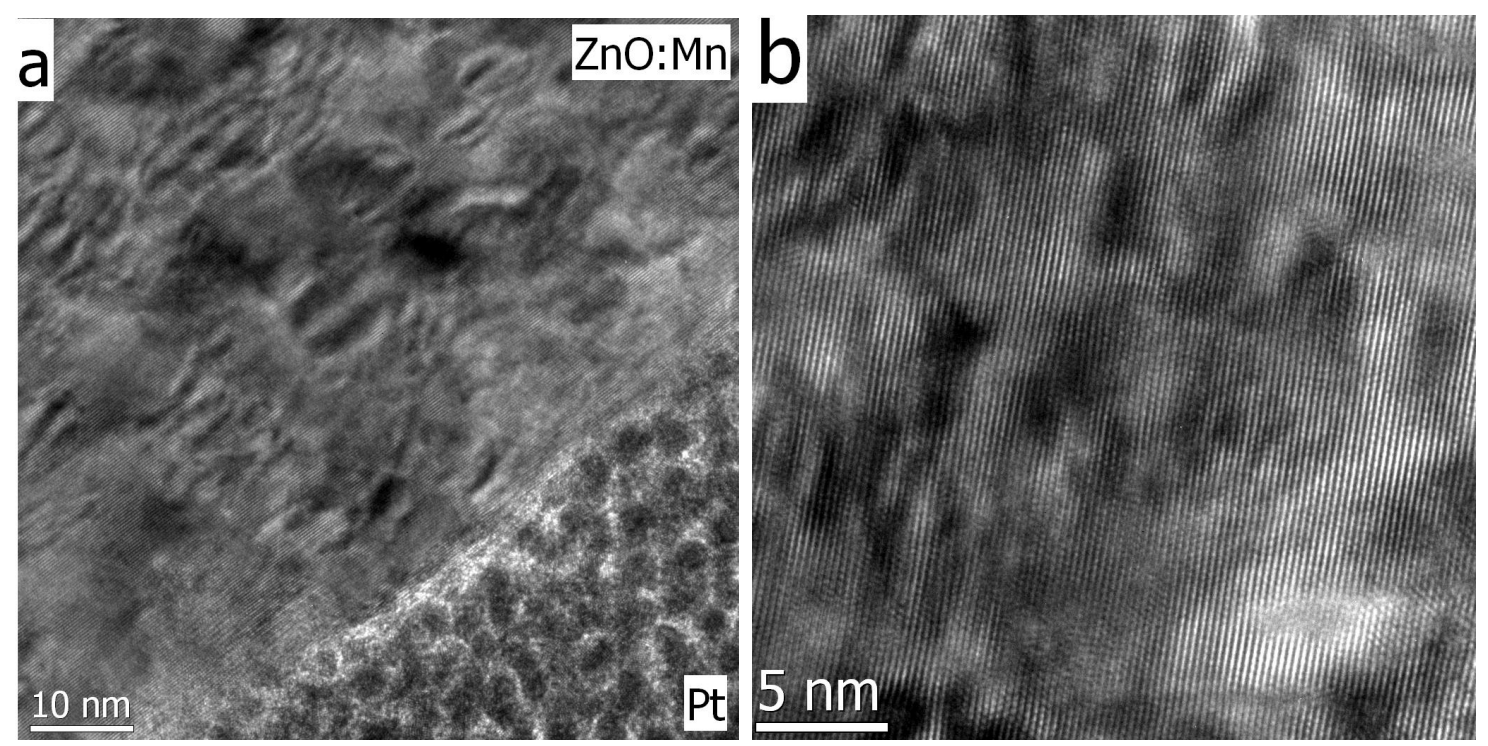

Figure 5.7: (a) Cross-section TEM and (b) HR-TEM micrographes of a 4 at.\% $M n$ implanted $Z n O$ single crystal show no secondary phase [325]. The TEM specimen was cutted out by FIB milling after annealing at $700^{\circ} \mathrm{C}$.

tectable in common XRD setups. Furthermore, phases could be formed, which are not Raman active such as crystals with cubic symmetry. Therefore, TEM specimen of the transition metal implanted $\mathrm{ZnO}$ single crystal samples were fabricated by FIB milling.

Figure 5.7 shows a TEM (a) and HR-TEM micrograph of the 4 at.\% Mn implanted $\mathrm{ZnO}$ single crystal after annealing at $700{ }^{\circ} \mathrm{C}$. All TEM studies show irregular bright and dark regions within the implantion depth as visible in figure 5.7a. The high resolution micrograph in figure 5.7b demonstrates the high defect density remaining in the $\mathrm{ZnO}$ lattice after annealing at $700{ }^{\circ} \mathrm{C}$. But no secondary phases are found in samples with transition metal concentrations below 8 at.\%, which is well in agreement with the XRD and Raman results.

As-grown $\mathrm{ZnO}$ nanowires are single crystalline and grow along the $\mathrm{ZnO}$ c-axis (see section 5.1 and references $[237 ; 326]$ ), where neither stacking faults nor any dislocations were observed. This changes completely after the ion implantation process and subsequent annealing and is illustrated in figure 5.8 [50]. The high-resolution (HR) TEM micrograph of a 4 at.\% Co implanted $\mathrm{ZnO}$ nanowire in figure 5.8a reveals an irregular contrast, where heavily damaged lattice regions appear as dark spots. The highlighted regions in figure $2 \mathrm{a}$ are magnified in figures $5.8 \mathrm{~b}$ (left region) and $5.8 \mathrm{c}$ (right region), respectively. Figure 5.8c shows a nearly perfect and regularly arranged $\mathrm{ZnO}$ lattice, in contrast to figure 5.8b. These micrographs display 


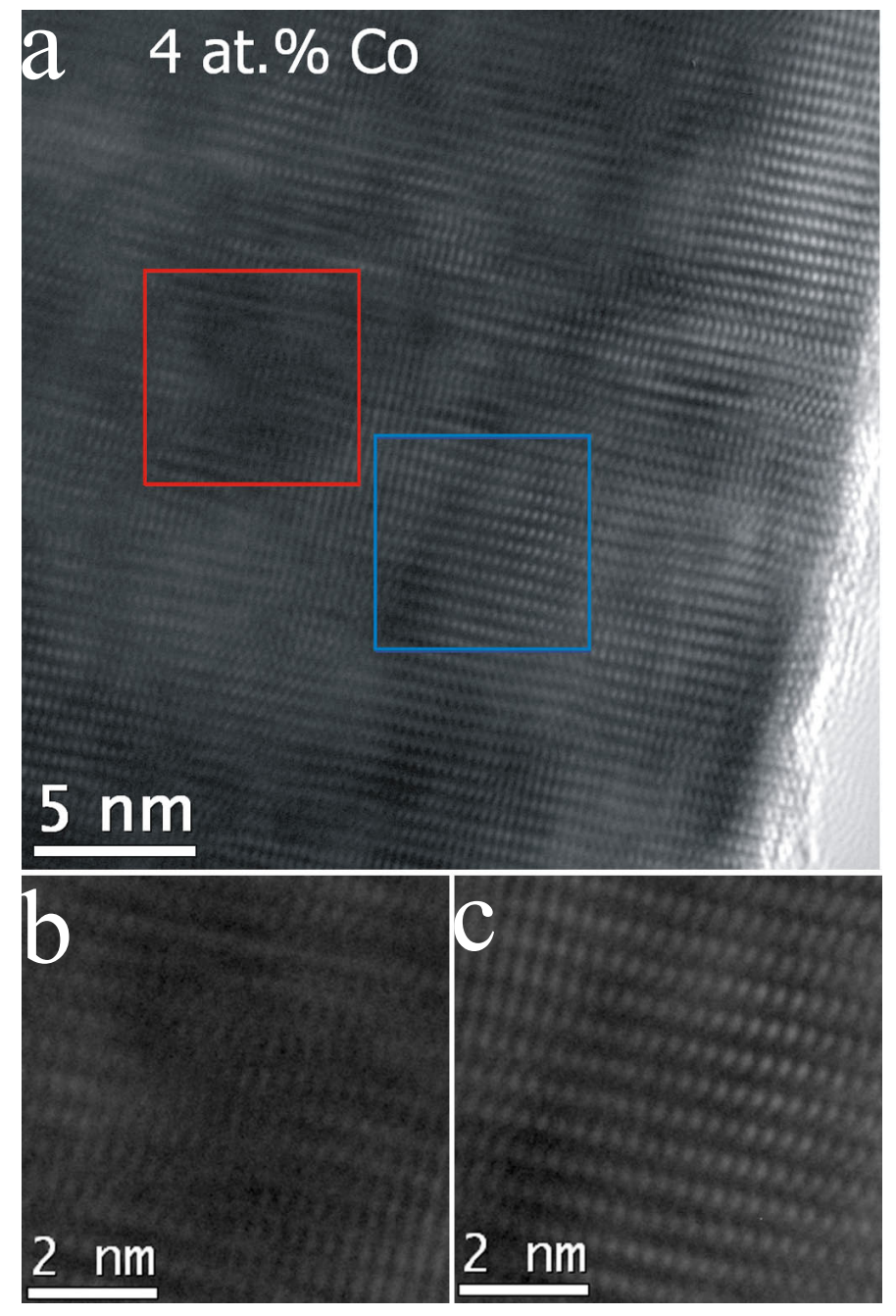

Figure 5.8: (a) High resolution TEM micrograph of a 4 at.\% Co implanted $\mathrm{ZnO}$ nanowire revealing a high damage remaining in the crystal after annealing, (b) and (c) are magnifications of the left and right marked regions in (a) [50], respectively.

the differences between a highly damaged region (5.8b) and slightly damaged region (5.8c). Nevertheless, there is no formation of new secondary phases as reported for TM implanted $\mathrm{ZnO}$ single crystals.

Irradiation of $\mathrm{ZnO}$ with electrons or ions generates a large number of dislocations and point defects $[318 ; 322]$ and subsequent annealing reduces the defect concentration. Yoshiie et al. had shown the formation of two different interstitial type dislocations in $\mathrm{ZnO}$ after electron irradiation with Burgers vector parallel to the c-axis or Burgers vectors perpendicular to the c-axis [322]. Experimental data reveal a low healing temperature for dislocations with Burgers vectors perpendicular to the c-axis [327], and should result in a higher annealing rate. However, the involved impurity complex is also a crucial factor for the annealing behaviour just as much as the type of dislocation [327]. Ion implantation of transition metals would result in high multiplicity of possibly involved defect complexes, such as: interstitialvacancies, interstitial-antisites or interstitial-interstitial defect complexes. Whereby 

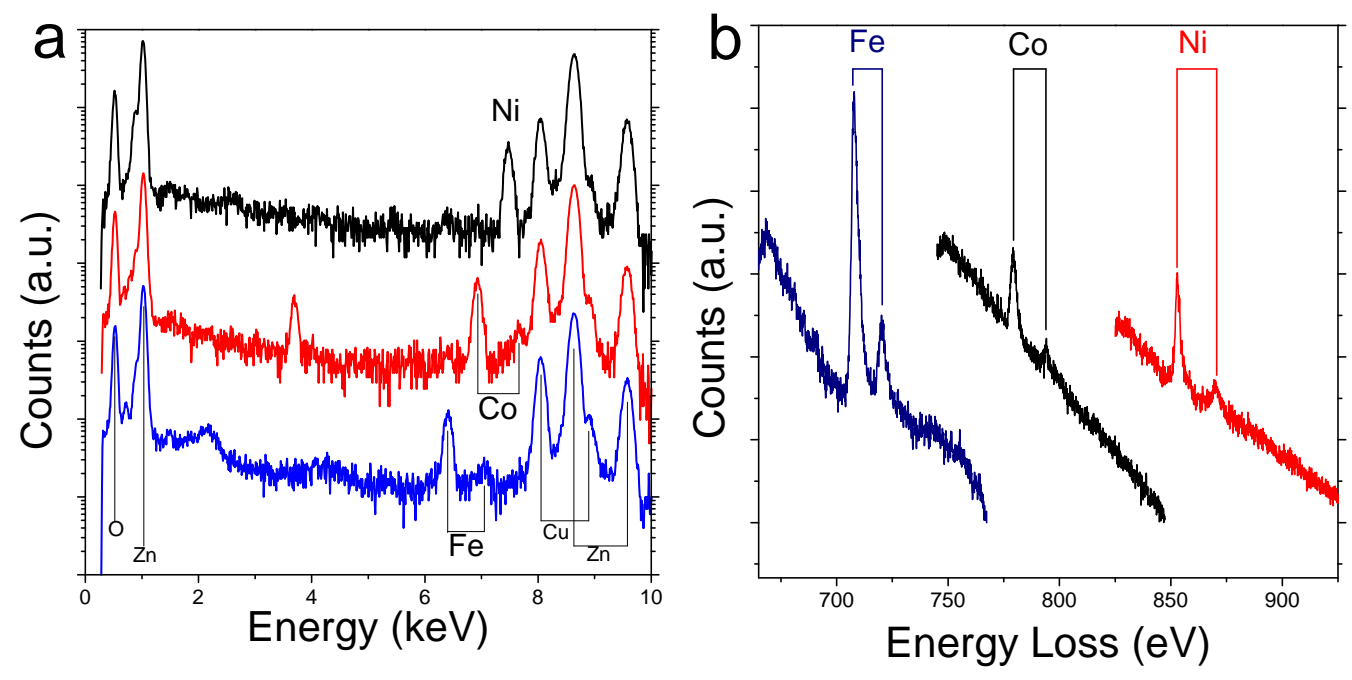

Figure 5.9: Composition after ion implantation and annealing was recorded with EDS (a) and EELS (b) on Fe, Co and Ni implanted ZnO nanowires with TM concentrations of 4 at. $\%$.

dislocations involving oxygen vacancies are removed by annealing at $300{ }^{\circ} \mathrm{C}$ [322]. $\mathrm{ZnO}$ exhibits a small activation energy for dislocation glide motion [328], but the activation energy and glide velocity of a dislocation depends strongly on the involved defect complexes. Dislocations could be annealed by sliding out of the surface or by mutual annihilation, whereby the latter one is only possible for dislocations with parallel arranged Burgers vectors with opposite sign [329]. Hence, highly immobile dislocation defect complexes would constrain the movement of mobile dislocations and result in the formation of regions with high dislocation densities as visible in figure 5.7 and 5.8. In the present study, we found that it was not possible to completely remove the structural damage created by the ion implantation process after annealing at $700{ }^{\circ} \mathrm{C}$ for 30 minutes.

The incorporation of the implanted TM was checked with EDS and EELS during TEM analysis. Figure 5.9 shows the spectra obtained for the different TM implanted and annealed $\mathrm{ZnO}$ nanowires with a nominal TM concentration of 4 at.\%. EDS spectra show clear signals of $\mathrm{Zn}$ and $\mathrm{O}$ from the analyzed $\mathrm{ZnO}$ nanowire and $\mathrm{Cu}$ signals from the TEM grid. According to the implanted TM ion, additional signals appear in all analysed nanowires, where representative spectra of $\mathrm{Fe}$, Ni and Co implanted $\mathrm{ZnO}$ nanowires are shown in figure 5.9a. Figure 5.9b shows representative EELS spectra of $\mathrm{Fe}, \mathrm{Ni}$ or $\mathrm{Co}$ implanted $\mathrm{ZnO}$ nanowires with their corresponding TM signals. All data reveal the incorporation with the desired concentration as well as no outdiffusion upon annealing. 

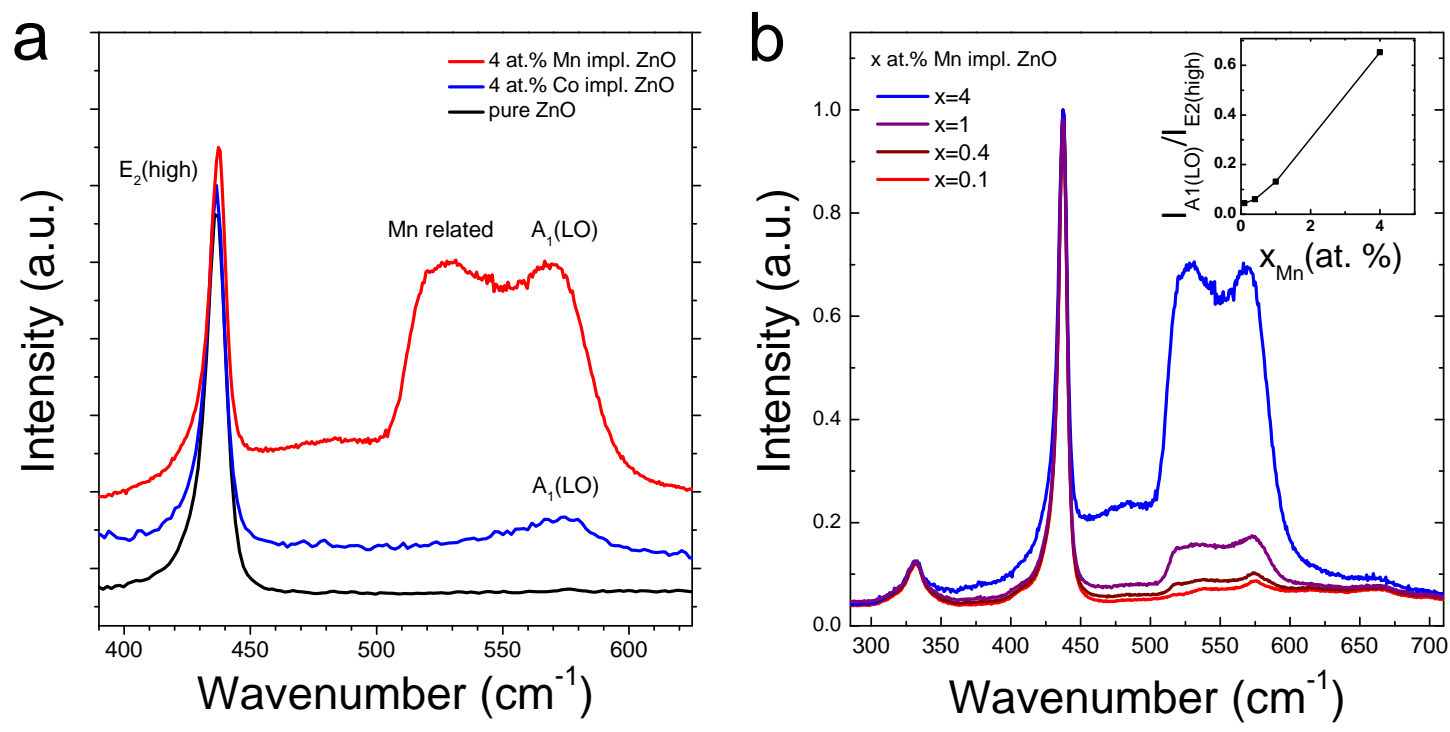

Figure 5.10: (a) Raman spectra of 4 at.\% $M n$ and 4 at.\% Co implanted $Z n O$ single crystals are compared to pure ZnO single crystals [325]. (b) Raman spectra of $M n$ implanted $Z n O$ reveal an increase of the $I_{A_{1}(L O)} / I_{E_{2}^{\text {high }}}$ ration (inset) with increasing Mn concentration [325].

\subsection{Local vibration modes of manganese in $\mathrm{ZnO}$}

Figure 5.10a shows the Raman spectra of pure $\mathrm{ZnO}$ and $\mathrm{ZnO}$ implanted with 4 at.\% Mn, compared to $\mathrm{ZnO}$ implanted with 4 at.\% Co [19]. In addition to the $\mathrm{ZnO} E_{2}^{\text {high }}$ mode and $A_{1}(L O)$ phonon band the $\mathrm{Mn}$ implanted $\mathrm{ZnO}$ samples show an broad band ranging from $500 \mathrm{~cm}^{-1}$ to $600 \mathrm{~cm}^{-1}$, whereby the shape of this band indicates the contribution of at least two different phonon bands. The phonon mode with higher Raman shift contributing to this band can be assigned to the ion implantation disorder induced $A_{1}(L O)$ phonon mode. The mode with lower Raman shift is controversially discussed in literature as partly or completely disorder related [136; 312; 330-332]. Figure 5.10b reveals an increase of the additional phonon band between $500 \mathrm{~cm}^{-1}$ and $600 \mathrm{~cm}^{-1}$ with increasing Mn concentration [19]. However, the $\mathrm{Mn}$ concetration by ion implantation can be increased by increase of the $\mathrm{Mn}$ ion fluence, which results in a rise of ion implantation induced damage. Of course, an increase of the $A_{1}(L O)$ phonon side band is observed in all other transition metal implanted $\mathrm{ZnO}$ single crystals with increasing concentration (see figure 5.6c). Otherwise, the increasing $\mathrm{Mn}$ concentration within the $\mathrm{ZnO}$ crystal can induce local vibration modes or disorder: Even if perfectly incorporated on Zn sites, the Mn ions induce disorder, inherent to the mixture of $\mathrm{Zn}$ and $\mathrm{Mn}$ on the cation sublattice. 

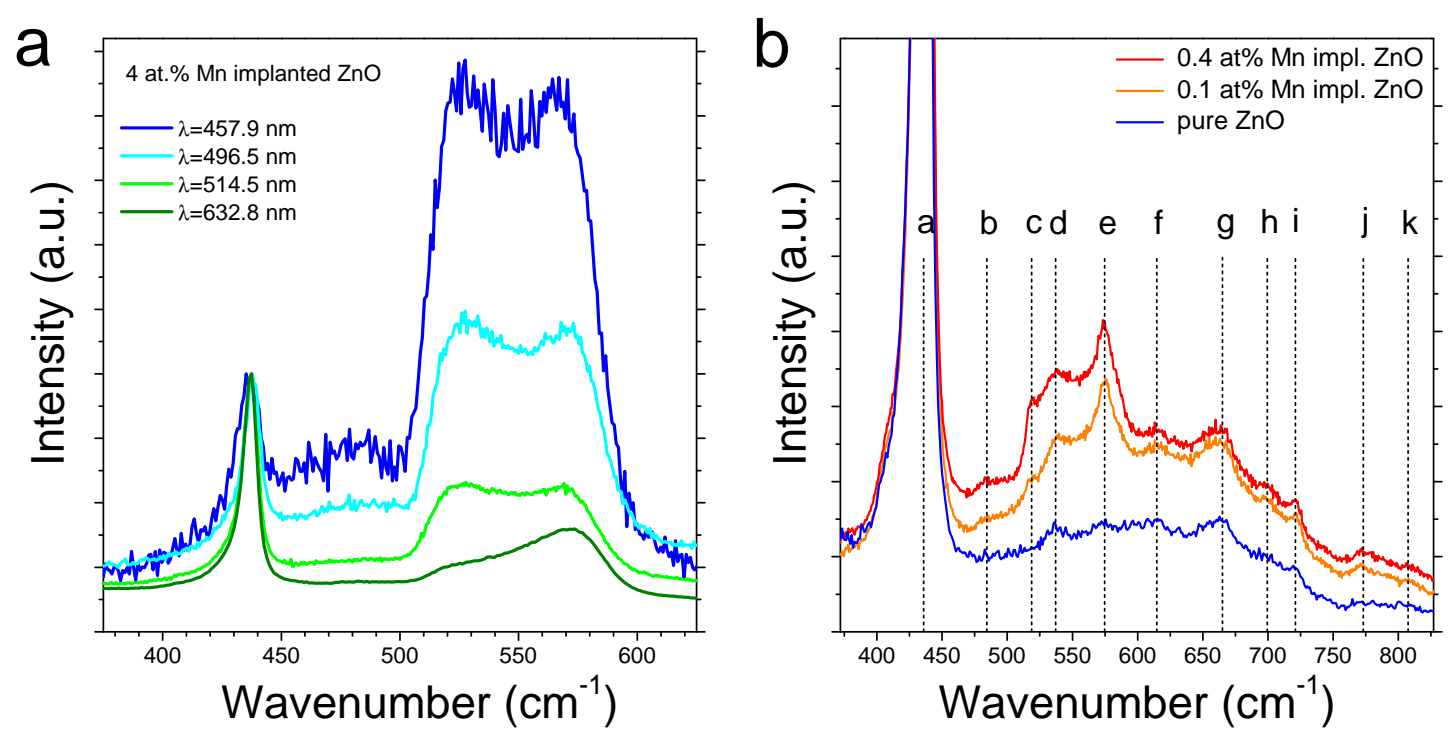

Figure 5.11: (a) shows the Raman resonance measurements of the 4 at.\% Mn implanted $\mathrm{ZnO}$ single crystal at room temperature (all spectra are normalized to the $E_{2}^{\text {high }}$ mode) [325]. (b) Detail comparision of broad band between $500 \mathrm{~cm}^{-1}$ and $600 \mathrm{~cm}^{-1}$ of $\mathrm{Mn}$ implanted $\mathrm{ZnO}$ with pure $\mathrm{ZnO}$ (at room temperature, excitation: $514.5 \mathrm{~nm}$ (Mn implanted $\mathrm{ZnO}$ ) and $632.8 \mathrm{~nm}$ (pure $\mathrm{ZnO})$ ) [325].

Resonance measurements using different laser excitation wavelengths are conducted to study the different contributions to the broad band between $500 \mathrm{~cm}^{-1}$ and $600 \mathrm{~cm}^{-1}$ in Raman spectra, which is shown in figure 5.11a. With decreasing excitation wavelength this broad band rises in intensity relative to the $E_{2}^{h i g h}$ phonon mode, which can be explained by impurity activated Fröhlich scattering [313]. The resonance effect is particularly strong for the phonon mode with lower Raman shift in the broad band. Due to the observations it can be supposed, that the Mn ion in $\mathrm{ZnO}$ plays a major role in the broad band between $500 \mathrm{~cm}^{-1}$ and $600 \mathrm{~cm}^{-1}$ besides the ion implantation damage, especially for the left shoulder.

Figure $5.11 \mathrm{~b}$ shows the broad band between between $500 \mathrm{~cm}^{-1}$ and $600 \mathrm{~cm}^{-1}$ of the 0.4 and 0.1 at.\% Mn implanted $\mathrm{ZnO}$ in more detail. The low $\mathrm{Mn}$ concentration and therefore lower disorder in those samples gives a higer possiblity to resolve separate modes instead of the broad unresolved band caused by the high phonon DOS in this region [333]. The features at about $437 \mathrm{~cm}^{-1}$ (labelled a) and $575 \mathrm{~cm}^{-1}$ (labelled e) can be attributed to the modes $E_{2}^{\text {high }}$ and $A_{1}(L O)$ mode, respectively. The mode at $483 \mathrm{~cm}^{-1}$ (labelled b) can be assigned to a multi-phonon process of ZnO [303]. All features registered between 600 and $825 \mathrm{~cm}^{-1}$, i.e. (f)-(k) in figure 5.11b, are also seen in the measurements for the pure $\mathrm{ZnO}$ substrate and can therefore be 


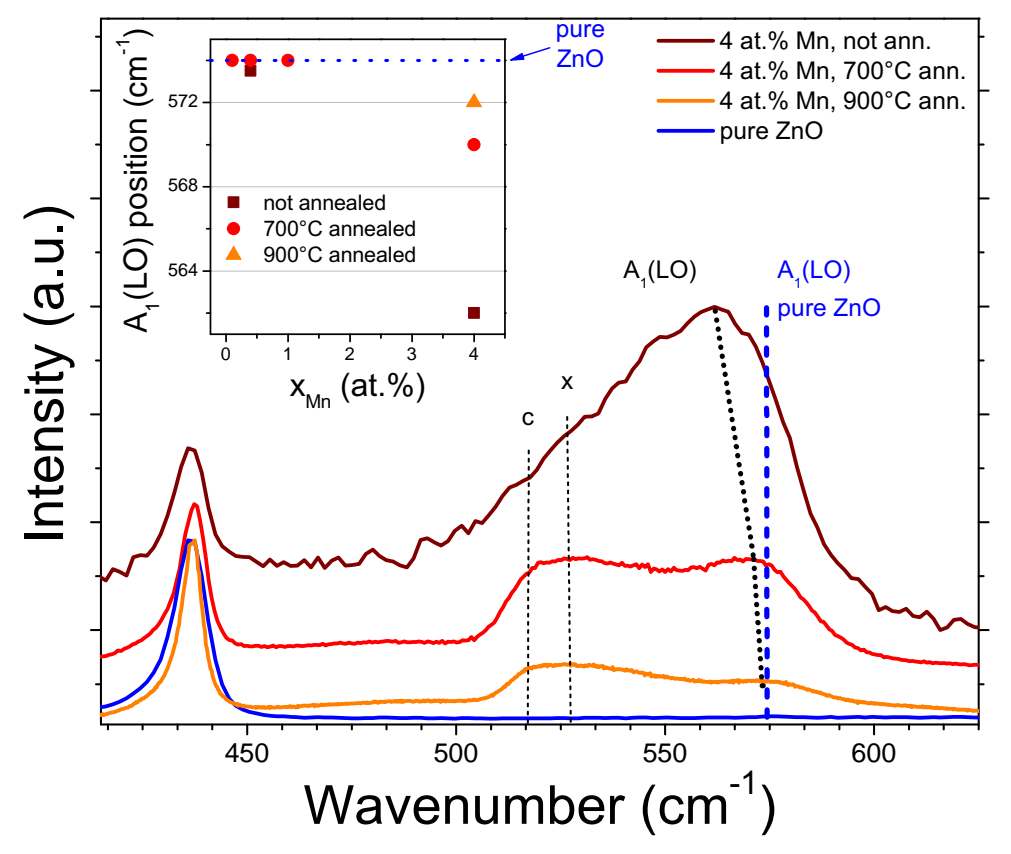

Figure 5.12: Raman spectra of the 4 at.\% Mn implanted samples shows the annealing behavior of the Raman mode at $519 \mathrm{~cm}^{-1}$ and $527 \mathrm{~cm}^{-1}$, which are labeled with $c$ and $x$ [325], respectively.

explained by multiphonon processes of Raman active modes from pure ZnO (see for instance table 5.1 and reference [303]). Some of these multiphonon modes are stronger in intensity in the $\mathrm{Mn}$ implanted samples than for pure $\mathrm{ZnO}$ due to disorder effects and impurity induced resonance. The mode labeled with $\mathrm{d}$ in figure $5.11 \mathrm{~b}$ at $\sim 537 \mathrm{~cm}^{-1}$ can be assignet to a $2 L A$ or to the $2 B_{1}^{\text {low }}$ process acording to Cusco et al. [303]. However, the mode at $\sim 519 \mathrm{~cm}^{-1}$ (labelled with c in figure $5.11 \mathrm{~b}$ ) can not be assigned to a $\mathrm{ZnO}$ phonon mode. Interestingly, this feature significantly increases in intensity with rising $\mathrm{Mn}$ concentration from the 0.1 at.\% Mn implanted sample to the 0.4 at.\% Mn implanted sample.

A further additional peak (which will be labeled as $\mathrm{x}$ ) was observed in bulk $\mathrm{Zn}_{0.96} \mathrm{Mn}_{0.04} \mathrm{O}$ grown via vapor transport at about $527 \mathrm{~cm}^{-1}$ [325]. Due to the high growth temperature during the vapor transport growth of $900{ }^{\circ} \mathrm{C}$ the $\mathrm{Mn}$ ions might be better substitutional incorporated in the $\mathrm{ZnO}$ lattice, therefore $\mathrm{Mn}$ implanted $\mathrm{ZnO}$ samples are further annealed at $900{ }^{\circ} \mathrm{C}$ in air. This annealing step results in a further reduction of the $A_{1}(L O)$ phonon mode and brings out a weak vibration mode at $527 \mathrm{~cm}^{-1}$ (labelled as $\mathrm{x}$ ) in the Raman spectra (see figure 5.12) [325]). Both modes, at $519 \mathrm{~cm}^{-1}$ and $527 \mathrm{~cm}^{-1}$, are reported in the literature for $\mathrm{Mn}$ alloyed $\mathrm{ZnO}$ 
and are taken as positive proof for the substitutional incorporation of $\mathrm{Mn}$ on zinc lattice sites $[312 ; 331 ; 332 ; 334]$. However, the mode at $527 \mathrm{~cm}^{-1}$ is very strong in the Raman spectra of high dose $\mathrm{Mn}$ implanted $\mathrm{ZnO}$ and decreases upon annealing (see figure 5.12). It should be noted that in $\mathrm{Sb}$ alloyed $\mathrm{ZnO}$ a phonon mode at $528 \mathrm{~cm}^{-1}$ was observed $[335 ; 336]$, which indicates that this mode can not be assigned to a Mn induced phonon mode. Though, this mode is possibly a $\mathrm{ZnO}$ mode, which is activated or intensified by $\mathrm{Mn}$ incorporation. Calculations of $B_{1}^{\text {low }}$ mode Raman shift by Manjon et al. show that the $2 B_{1}^{\text {low }}$ mode lies in this spectral region [337]. The $519 \mathrm{~cm}^{-1}$ mode can only be seen in low dose Mn implanted $\mathrm{ZnO}$ samples and in high dose $\mathrm{Mn}$ implanted $\mathrm{ZnO}$ samples after thermal annealing at $900{ }^{\circ} \mathrm{C}$, because of the strong and broad disorder band. In low dose $\mathrm{Mn}$ implanted $\mathrm{ZnO}$ samples, the intensity of this mode scales with the Mn ion concentration (see figure 5.11b). By electron paramagnetic resonance measurements, it is shown, that the $\mathrm{Mn}$ ions are incorporated on a zinc lattice site after thermal annealing at $700{ }^{\circ} \mathrm{C}$ [325]. The phonon mode at $519 \mathrm{~cm}^{-1}$ is a candidate for a local vibration mode of $\mathrm{Mn}$ ions on zinc lattice site in $\mathrm{ZnO}$.

The Raman spectra of $\mathrm{Fe}, \mathrm{Ni}$ and $\mathrm{Co}$ implanted $\mathrm{ZnO}$ reveal no additional phonon mode, which can be assigned to a local vibration mode of the corresponding transition metal ion in ZnO. This observation is contradicting the results of Sudakar et al., who had assigned an additional phonon mode at $690 \mathrm{~cm}^{-1}$ to a local vibration mode of $\mathrm{Co}$ in $\mathrm{ZnO}$ [338].

\subsection{Secondary phase formation}

In high dose transition metal implanted $\mathrm{ZnO}$ some new signals are observable in $\mathrm{XRD}$ and/or Raman spectra after annealing at $700{ }^{\circ} \mathrm{C}$ in air. Figure 5.13 shows the development of $\mathrm{Ni}$ implanted $\mathrm{ZnO}$ single crystals after annealing at $700{ }^{\circ} \mathrm{C}$, where additional diffraction peaks appear above 4 at.\%. These additional XRD diffraction peaks as well as all additional Raman signals do not fit to typical signals of $\mathrm{ZnO}$. A low resolution TEM micrograph of the 16 at.\% Mn implanted $\mathrm{ZnO}$ single crystals in figure 5.14 reveals some bright regions within $\mathrm{ZnO}$, which are $20 \mathrm{~nm}$ x $100 \mathrm{~nm}$ in size. All these bright regions are alligned parallel to the surface of the $\mathrm{ZnO}$ single crystal. Starting from the surface of the single crystal an EDS line scan was performed along the TEM cross section of the 16 at.\% Mn implanted $\mathrm{ZnO}$ single crystals and is indicated by the red line in figure 5.14. The EDS spectrum is shown in figure 5.14 , whereby the zinc $L \alpha$, oxygen $K \alpha$, and the manganese $K \alpha \& K \beta$ X-ray lines 


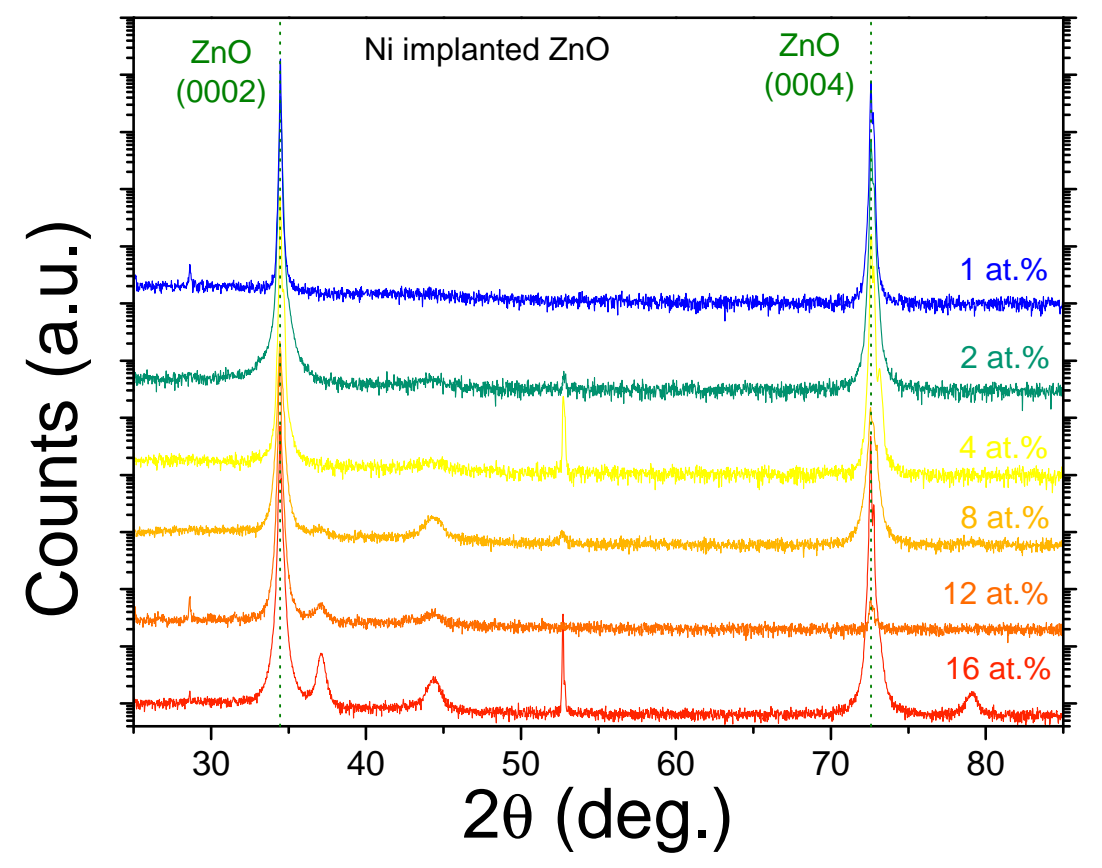

Figure 5.13: XRD spectra of Ni implanted ZnO single crystals after annealing at $700{ }^{\circ} \mathrm{C}$ show additional diffractions for all samples with a Ni concentration above 4 at.\%. The peak marked with an asterisk is attributed to the (0003) peak of ZnO.

are presented. Several slumps can be observed in the zinc $K \alpha$ alongside the scan direction. On the other hand, the manganese $K \alpha$ and $K \beta$ lines show break-outs at the same scanning depth where the slumps in the zinc $L \alpha$ line appear. Also the oxygen $K \alpha$ line shows such break-outs at the same position along the scanning direction, but these break-outs are rather weak. The break-outs and slumps in the $\mathrm{X}$-ray lines of the EDS line scan take place as soon as the exciting electron beam crosses the bright regions in the TEM micrograph. This observation confirms the formation of $\mathrm{Mn}$ rich and $\mathrm{Zn}$ depleted seggregations, which appear as bright regions in the TEM micrograph in figure 5.14. These segregations may contain a slightly higher oxygen concentration, as indicated by the break-outs in the EDS line scan.

The formation of secondary phases are observed in all high dose transition metal implanted $\mathrm{ZnO}$ single crystals after annealing. Within this chapter of this thesis the chemical nature of these secondary phases are delimitated as far as possible, also the limitations of all techniques used to delimitate the segregations are presented. 


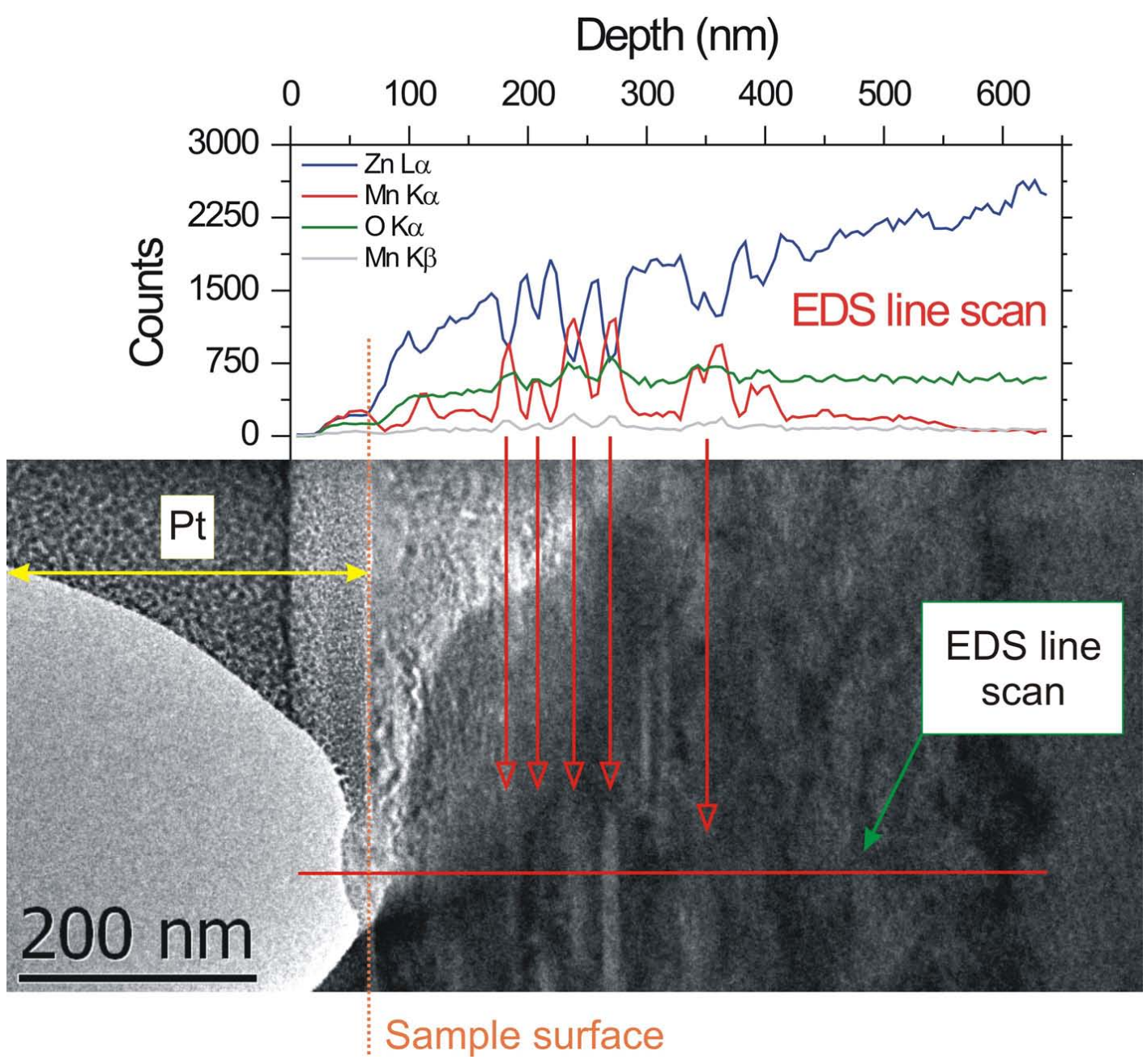

Figure 5.14: The cross section of a 16 at.\% Mn implanted $Z n O$ single crystal after annealing at $700^{\circ} \mathrm{C}$ reveal parallel arranged bright segregations. The EDS line scan reveal several slums in the zinc $L \alpha$ line and break-outs manganese $K \alpha$ and $K \beta$ lines.

\subsection{1 $\mathrm{ZnMn}_{2} \mathrm{O}_{4}$ in $\mathrm{Mn}$ implanted $\mathrm{ZnO}$}

Optical microscope ${ }^{3}$ images show small inclusions with some micrometer size in all $\mathrm{Mn}$ implanted $\mathrm{ZnO}$ single crystals with $\mathrm{Mn}$ concentrations of $\geq 8$ at.\%. Lateral mapping of the inclusions show an increase in the coverage with increasing $\mathrm{Mn}$ concentration from about $0.1 \%$ in the 8 at.\% Mn implanted $\mathrm{ZnO}$ sample to about $30 \%$ in the 16 at.\% $\mathrm{Mn}$ implanted $\mathrm{ZnO}$ sample (see microscope image in figure 5.15 [19]). In the 8 at.\% Mn implanted $\mathrm{ZnO}$ sample these inclusions appear orange under the light microscope and all inclusions are randomly distributed over the

\footnotetext{
${ }^{3}$ Both Raman setups are equipped with an optical microscope.
} 


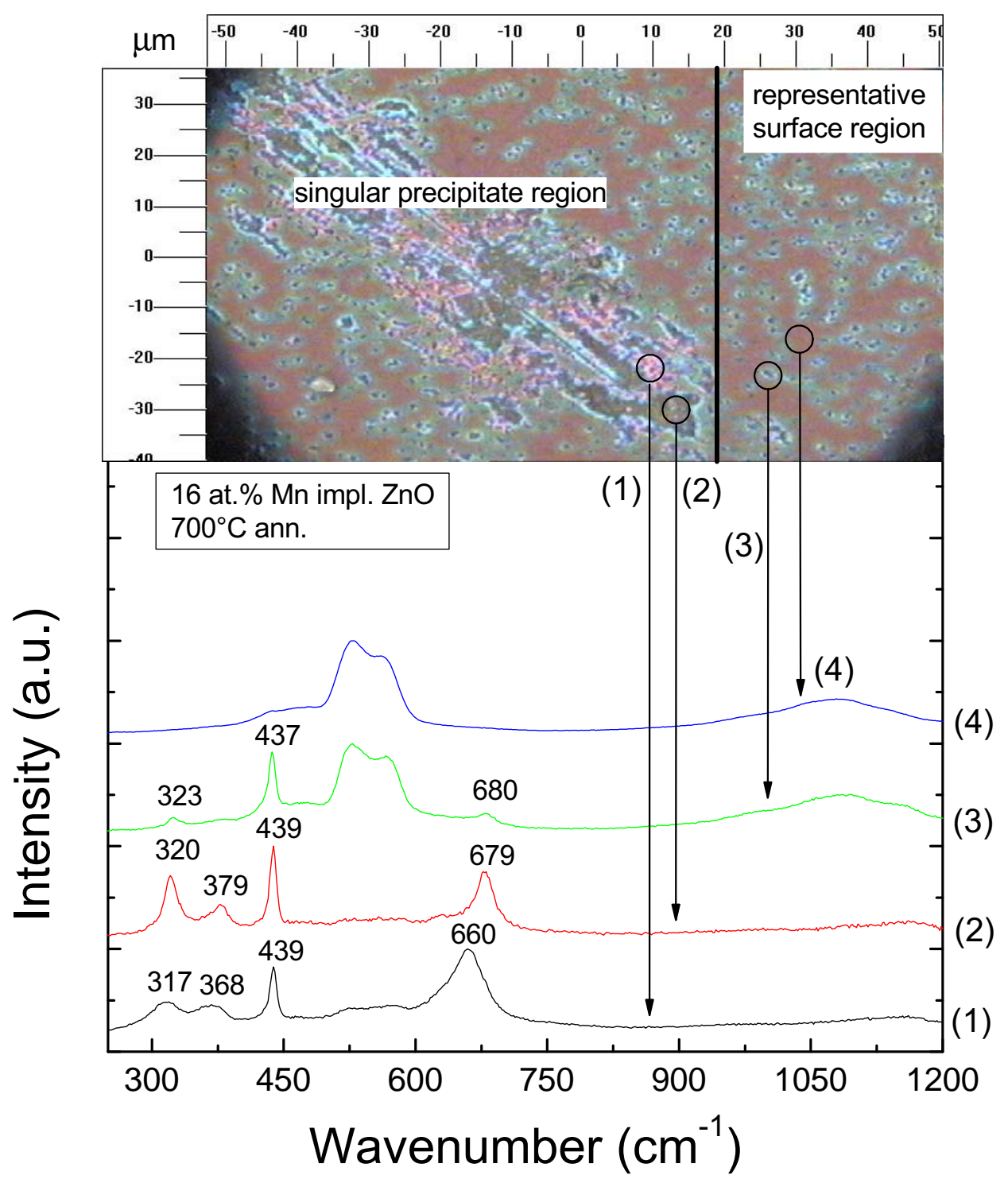

Figure 5.15: The lateral inhomogeneity of the 16 at.\% Mn implanted $\mathrm{ZnO}$ single crystal due to precipitate formation, demonstrated by micro-Raman spectra taken after $700^{\circ} \mathrm{C}$ annealing (excitation: $514.5 \mathrm{~nm}$ ) [19]. The laser focus positions are marked in the optical microscope picture. Spectra from bottom to top: laser focused on (1) violet spot and (2) dark spot in the shown singular precipitate area; laser focused on (3) the green island and (4) the red-coloured region, representative for most of the surface region.

whole sample surface. Both samples with higher Mn concentrations show precipitate regions in which the density of the inclusions is higher. However, with the optical microscope image in figure 5.15 of the 16 at.\% Mn implanted $\mathrm{ZnO}$ the crystal can be devided into four different $\mathrm{ZnO}: \mathrm{Mn}$ domains, which are indicated in figure 5.15 
by small circles. The Raman spectra from those domains differ from each other. The Raman spectrum of the red domain [spectrum (4) in figure 5.15] shows the typical Raman spectrum of 'low' dose Mn implanted ZnO samples with the strong local vibration mode and disorder band between $500 \mathrm{~cm}^{-1}$ and $600 \mathrm{~cm}^{-1}$. All other three domains reveal three additional Raman signals at about $320 \mathrm{~cm}^{-1}, 380 \mathrm{~cm}^{-1}$, and $680 \mathrm{~cm}^{-1}$ (see spectra (1)-(3) in figure 5.15). The spectral position as well as the intensity ratios of these three signals are characteristic for $\mathrm{ZnMn}_{2} \mathrm{O}_{4}$ [136]. The comparision of the three Raman spectra (1)-(3) in figure 5.15 reveals slight differences in the spectral positions of those three lines. A line shift of Raman features can be induced by strain effects. The red shift of up to $10 \mathrm{~cm}^{-1}$ indicates a tensile strain affecting the lattice of the $\mathrm{ZnMn}_{2} \mathrm{O}_{4}$ secondary phases in $\mathrm{Mn}$ implanted $\mathrm{ZnO}$, which could be induced by a lattice mismatch between the $\mathrm{ZnMn}_{2} \mathrm{O}_{4}$ secondary phases and the $\mathrm{Mn}$ implanted $\mathrm{ZnO}$ matrix. Otherwise, for $\mathrm{Mn}_{3} \mathrm{O}_{4}$ Raman lines at about $310-315 \mathrm{~cm}^{-1}, 370 \mathrm{~cm}^{-1}$, and $650 \mathrm{~cm}^{-1}$ are reported in the literature [339], and therefore, the shift of those lines can also be explained by non-shoichometric phases of $\mathrm{Zn}_{x} \mathrm{Mn}_{3-x} \mathrm{O}_{4}$.

Furthermore the $E_{2}^{\text {high }}$ phonon mode of $\mathrm{ZnO}$ is observable in all three spectra, whereby the disorder band between $500 \mathrm{~cm}^{-1}$ and $600 \mathrm{~cm}^{-1}$ can only be observed in the spectrum (3) and is weakly indicated in spectrum (1). This observation indicates the self-purification process of $\mathrm{ZnO}$ by formation of secondary phases. Domains with a high density of inclusions reveal a less disordered ZnO Raman spectrum with the additional intensive Raman signals of $\mathrm{Zn}_{x} \mathrm{Mn}_{3-x} \mathrm{O}_{4}$ [136; 339].

Figure 5.16 shows the X-ray diffraction spectra for the 16 at.\% Mn implanted $\mathrm{ZnO}$ single crystal after annealing at $700{ }^{\circ} \mathrm{C}$ recorded with a conventional XRD setup (Brucker AXS D8 Discover) in (a) and after annealing at $900{ }^{\circ} \mathrm{C}$ recorded with a syncrotron XRD beam (DESY Hamburg) ${ }^{4}$ in (b). Besides the strong diffraction peaks of $\mathrm{ZnO}$ some new diffractions are observable in the 16 at.\% Mn implanted $\mathrm{ZnO}$ after annealing (see figure 5.16a), which are visible for all $\mathrm{Mn}$ implanted $\mathrm{ZnO}$ single crystals with a Mn concentration $\geq 8$ at.\%. These additional diffractions are located at about 35.8 deg., 36.9 deg. (see inset in figure 5.16a), 56.8 deg. and 75.9 deg.. Within figure 5.16a possible secondary phases are indicated, whereby the diffraction angle of the corresponding phase diffraction peak were taken from [275]. During the Raman measurements, the $\mathrm{Zn}_{x} \mathrm{Mn}_{3-x} \mathrm{O}_{4}$ phase was identified as secondary phase for all $\mathrm{Mn}$ implanted $\mathrm{ZnO}$ single crystals with $\mathrm{Mn}$ concentrations $\geq 8$ at. $\%$. The diffraction peaks at $36.9 \mathrm{deg}$. (see inset in figure $5.16 \mathrm{a}$ ) and $56.8 \mathrm{deg}$. correspond

\footnotetext{
${ }^{4}$ In collaboration with E. Dynowska, Z. Golacki, W. Szuszkiewicz from the Institute of Physics, Polish Academy of Sciences, Warszawa, Poland.
} 

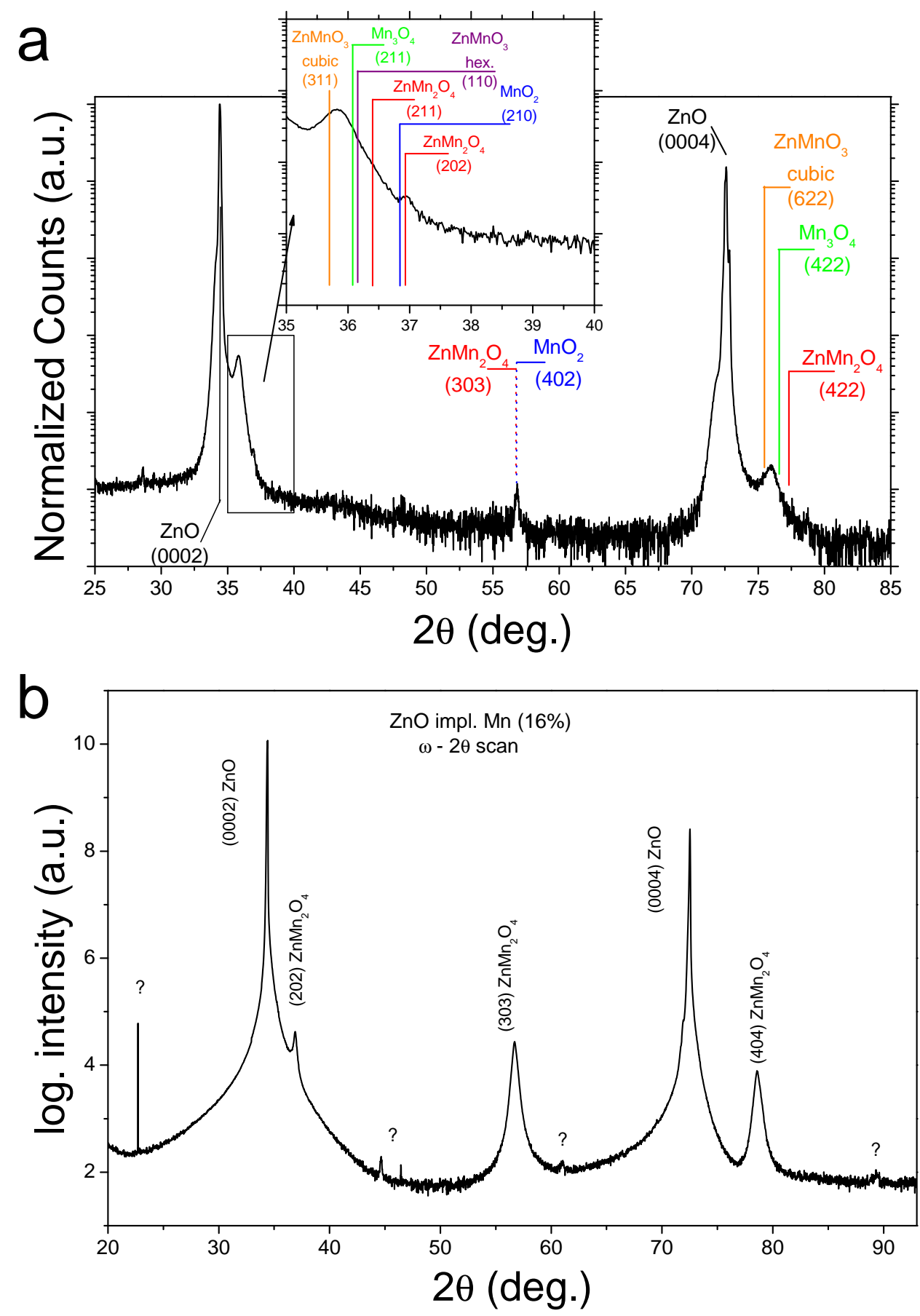

Figure 5.16: XRD diffractogram of the 16 at.\% Mn implanted $\mathrm{ZnO}$ single crystal after $700^{\circ} \mathrm{C}(\mathrm{a})$ and $900^{\circ} \mathrm{C}(\mathrm{b})$ annealing. Besides the (0002) and (0004) diffractions of the $\mathrm{ZnO}$ host, new additional features are observed after annealing at $700^{\circ} \mathrm{C}$ and $900^{\circ} \mathrm{C}$. These diffraction lines can be assigned to different $\mathrm{Mn}$ rich phases. 
very well to the literature values of the (202) and (303) Bragg peaks of $\mathrm{ZnMn}_{2} \mathrm{O}_{4}$ (hetaerolite, tetragonal lattice structure) [275], respectively. Also cubic $\mathrm{MnO}_{2}$ can be assigned to these diffraction peaks. Though $\mathrm{MnO}_{2}$ is not Raman active, one can not exclude the formation of $\mathrm{MnO}_{2}$

These diffraction peaks are fitted with Gaussian functions (equation 4.3 in section 4.2) for the determination of the full width at half maximum (FWHM, equation 4.4). With the FWHM value one can derive the size of the segregations via the Scherrer relation (given in equation 4.2) [276]. With this relation the size of the $\mathrm{ZnMn}_{2} \mathrm{O}_{4}$ segregations can be estimated to $(15 \pm 2) \mathrm{nm}$.

The inset in figure 5.16a shows perfectly the complexity of the determination of secondary phases of transition metal alloyed $\mathrm{ZnO}$. The diffraction peaks at about $35.8 \mathrm{deg}$. and $75.9 \mathrm{deg}$. must have the same origin, whereby the diffraction at 75.9 deg. must be the second order of the diffraction at the angle of $35.8 \mathrm{deg}$.. The reported (211) Bragg angle of the $\mathrm{ZnMn}_{2} \mathrm{O}_{4}$ and $\mathrm{Mn}_{3} \mathrm{O}_{4}$ phase is bigger than the observed diffraction at 35.8 deg., which is also the case for the (422) Bragg angle of both phases and the diffraction at $75.9 \mathrm{deg}$. [275]. On the other hand, the cubic $\mathrm{ZnMnO}_{3}$ phase has a Bragg angle of $35.7 \mathrm{deg}$. [275], which is smaller than the observed diffraction. However, strain within the segregations can induce a deformation of the lattice, which results in a shift of the diffraction peaks. Tensile strain would result in a shift to lower diffraction angles due to a larger distance between the lattice plains. For compressive strain an opposite behavior should be observed. Even though, this explains the differences between the observed diffraction angle and the database values for all three phases, one should raise the following question. All additional observed Raman signals are assigned up to now to $\mathrm{ZnMn}_{2} \mathrm{O}_{4}, \mathrm{Mn}_{3} \mathrm{O}_{4}$ and in-between those two phases, but where are the Raman signals of $\mathrm{ZnMnO}_{3}$ ? That is a question which can not be answered up to now, because no Raman identification of $\mathrm{ZnMnO}_{3}$ was reported, yet.

Hetaerolite $\left(\mathrm{ZnMn}_{2} \mathrm{O}_{4}\right)$ and hausmannite $\left(\mathrm{Mn}_{3} \mathrm{O}_{4}\right)$ have a tetragonal lattice structure with slightly different lattice constants [275]. As long as the $\mathrm{Zn}^{2+}$ ion does not replace an $\mathrm{Mn}^{3+}$ ion in the octahedral site of hausmannite a regular solid solution behavior should be observable [340], and therefore stable intermediate phases of the system $\mathrm{Zn}_{x} \mathrm{Mn}_{3-x} \mathrm{O}_{4}$ should exist. The diffraction peaks of those intermediate phases should be in-between the diffraction peaks of hausmannite and hetaerolite (for example: $2 \theta_{\text {hausmannite }, 211}=36.09 \leq 2 \theta_{\text {intermediate }, 211} \leq 36.525=2 \theta_{\text {hetaerolite }, 211}$ [275]). The same observation should be made for the Raman signals of the intermediate phase. After annealing at $900{ }^{\circ} \mathrm{C}$ in air the XRD spectrum shows only the additional diffraction peaks of $\mathrm{ZnMn}_{2} \mathrm{O}_{4}$ (see figure 5.16). The positions of the 

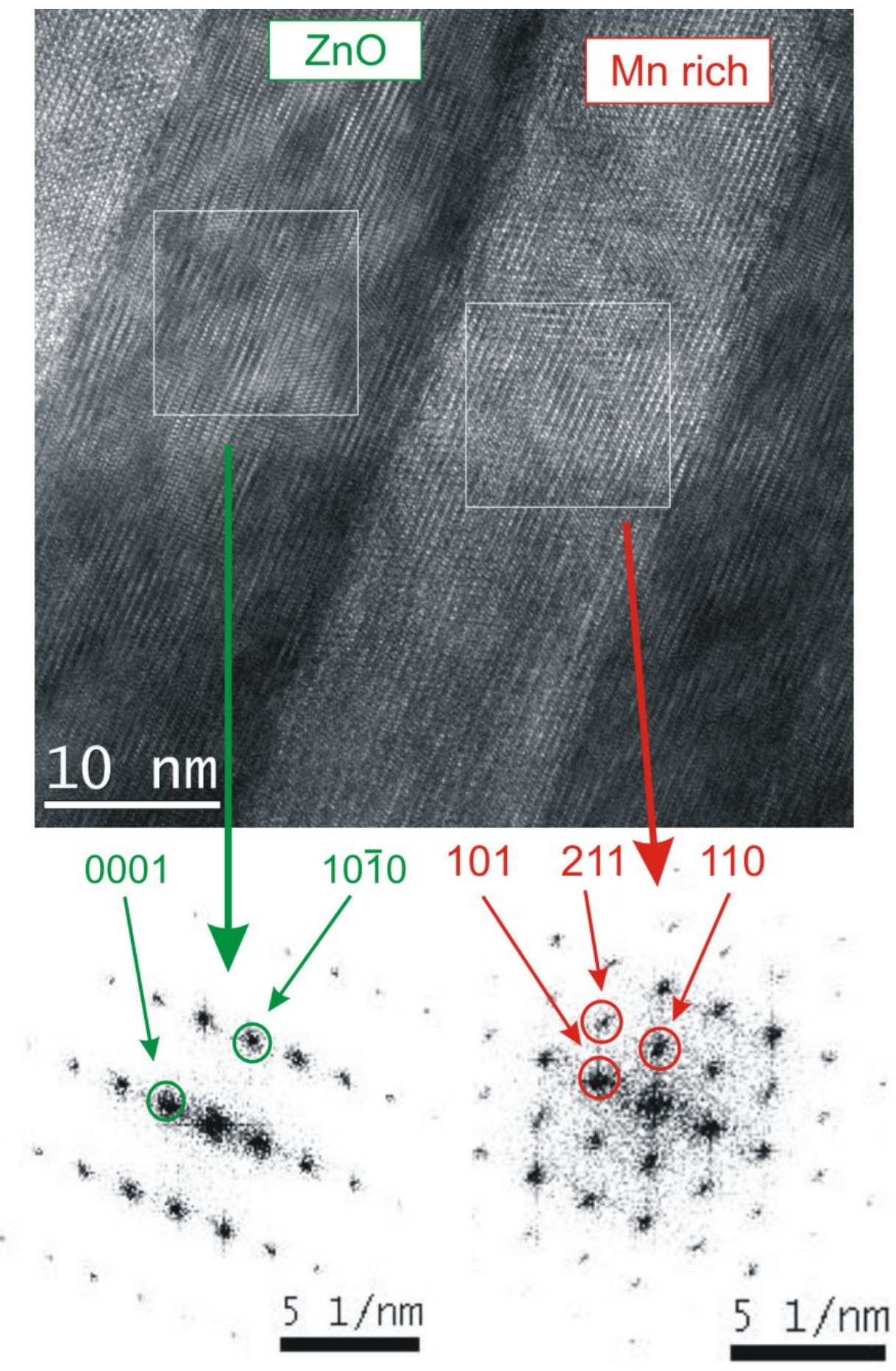

Figure 5.17: The high resolution TEM micrograph presents a Mn rich segregation in the 16 at.\% Mn implanted $\mathrm{ZnO}$ after annealing at $900{ }^{\circ} \mathrm{C}$. The FFT of the highlighted region in $\mathrm{ZnO}$ is shown on the left hand side and on the right hand side the FFT of the Mn rich segregation. 
diffraction peaks fit perfectly to the values given in the database [275], suggesting a reduction of the strain in the secondary phase clusters.

Figure 5.16b suggests that after annealing at $900{ }^{\circ} \mathrm{C}$ the [101] lattice direction of the $\mathrm{ZnMn}_{2} \mathrm{O}_{4}$ segregations are parallel oriented to the c-axis of $\mathrm{ZnO}$. Figure 5.17 shows a HR-TEM micrograph of the 16 at.\% Mn implanted $\mathrm{ZnO}$ samples after annealing at $900{ }^{\circ} \mathrm{C}$. Within the TEM micrograph in figure 5.17 bright and dark regions are visible, which corespond to the $\mathrm{Mn}$ rich regions (bright) and Mn poor regions (dark) of the low magnified TEM image in figure 5.14. To avoid confusion the $\mathrm{Mn}$ poor region is indicated as $\mathrm{ZnO}$ in figure 5.17, but it is necessary to note that this region is also $\mathrm{Mn}$ alloyed $\mathrm{ZnO}$. The fast Fourier transformed (FFT) images of the Mn poor and Mn rich regions are shown in figure 5.17 on the left hand side and right hand side, respectively. The FFT of the Mn rich phase is consistent with an electron diffraction pattern of a tetragonal lattice arrangement with zone axis [1 $\overline{1} \overline{1}]$ [294]. The distances of the marked diffraction spots to the central spot in the Mn rich FFT correspond to the lattice spacing of the (110) and (211) lattice planes of $\mathrm{ZnMn}_{2} \mathrm{O}_{4}$. A comparision of the lattice directions via the FFTs in figure 5.17 reveal a parallel arrangeent of the [0001] axis of $\mathrm{ZnO}$ and [101] direction of $\mathrm{ZnMn}_{2} \mathrm{O}_{4}$, and a parallel arrangement of the $[10 \overline{1} 0] \mathrm{ZnO}$ lattice direction with the $[12 \overline{1}]$ lattice direction of $\mathrm{ZnMn}_{2} \mathrm{O}_{4}$.

\subsection{2 $\mathrm{ZnCo}_{2} \mathrm{O}_{4}$ and $\mathrm{CoO}$ in $\mathrm{Co}$ implanted $\mathrm{ZnO}$}

The 16 at.\% Co implanted $\mathrm{ZnO}$ single crystal shows after annealing at $700{ }^{\circ} \mathrm{C}$ some new features in the Raman spectra (see figure 5.18) and some additional X-ray diffraction peaks (see figure 5.19) [19]. However, all other Co implanted samples show no additional feature in their Raman spectra, in contrast to the observations for $\mathrm{Mn}$ implanted $\mathrm{ZnO}$ and the data obtained by X-ray diffraction. The latter one shows new diffraction for Co implanted $\mathrm{ZnO}$ single crystals for Co concetrations $\geq 8$ at. $\%$.

Figure 5.18 shows the Raman spectra recorded at four different points (A-D) on the 16 at.\% Co implanted $\mathrm{ZnO}$ single crystal after annealing at $700{ }^{\circ} \mathrm{C}$. For all four points the images recorded with the optical mircoscope are shown in figure 5.18. All four points show a different look, which is also reflected in the Raman spectra of the corresponding points. Within the Raman spectra of point A and B no additional phonon mode other than the $A_{1}(L O)$ disorder band can be observed (see figure 5.18) and is contrary to the Raman spectra of point $\mathrm{C}$ and D. Point $\mathrm{C}$ and D show five additional phonon bands at $487 \mathrm{~cm}^{-1}, 524 \mathrm{~cm}^{-1}, 624 \mathrm{~cm}^{-1}, 692 \mathrm{~cm}^{-1}$, 


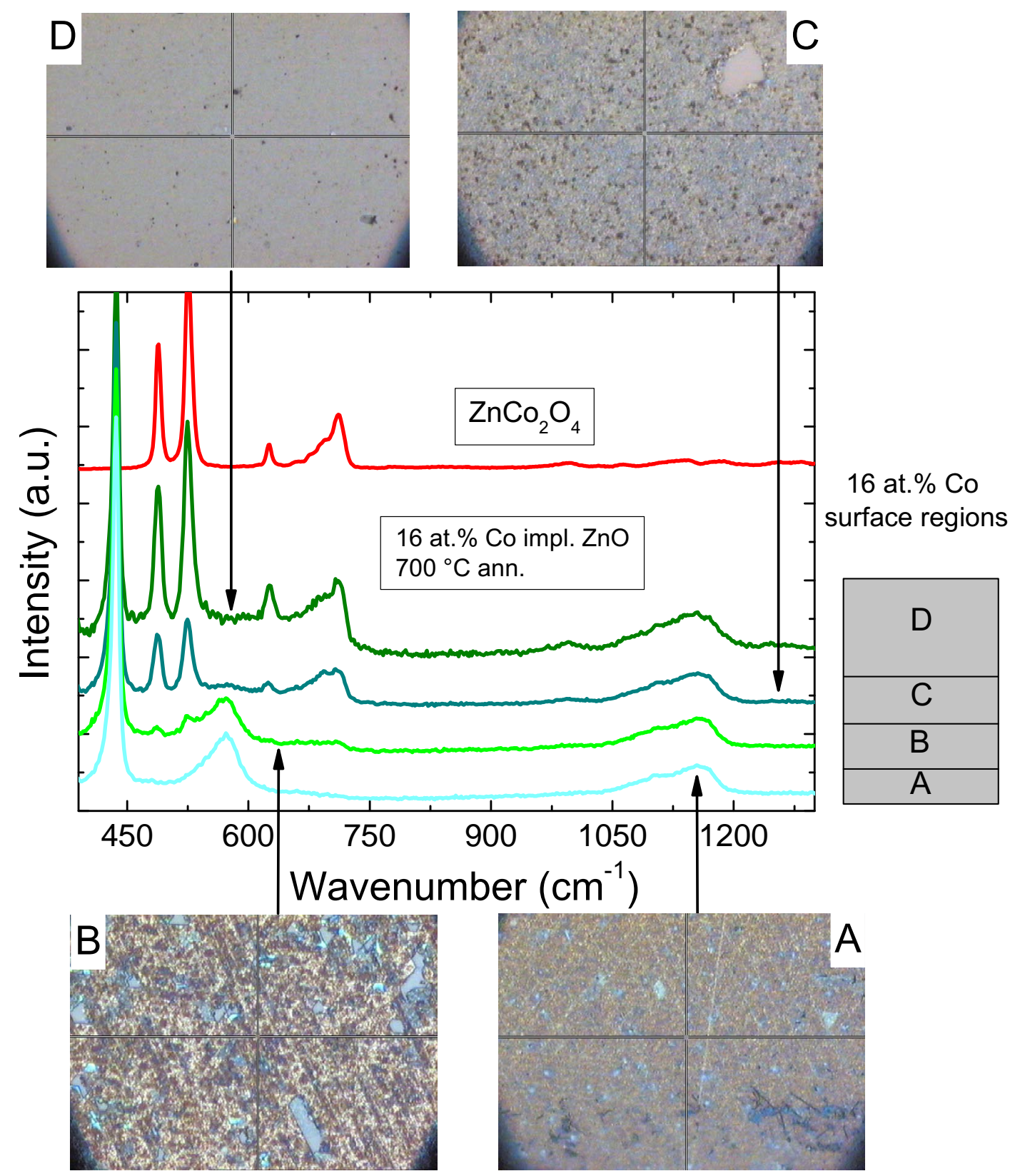

Figure 5.18: Micro-Raman spectra of different spots on the 16 at.\% Co implanted $\mathrm{ZnO}$ after $700^{\circ} \mathrm{C}$ annealing (excitation: $514.5 \mathrm{~nm}$ ) [19]. The Raman spectrum of a bulk ZnCo2O4 bulk sample is shown for comparison. The optical microscope pictures show the studied surface regions $A, B, C, D$ of this sample with inhomogeneously distributed secondary phase formation. Spectra from bottom to top: laser focused in the middle of the surface region (A), (B), (C), and (D). 
and $709 \mathrm{~cm}^{-1}$, which can not be assigned to $\mathrm{ZnO}$ phonon modes. For comparision the Raman spectrum of a ploycrystalline bulk $\mathrm{ZnCo}_{2} \mathrm{O}_{4}$ sample is added in figure 5.18. The phonon modes of the bulk $\mathrm{ZnCo}_{2} \mathrm{O}_{4}$ sample fit very well to the additional phonon mode of point $\mathrm{C}$ and $\mathrm{D}$ of the 16 at.\% Co implanted $\mathrm{ZnO}$ single crystal. Therefore, the Raman spectra indicate the formation of $\mathrm{ZnCo}_{2} \mathrm{O}_{4}$ in the 16 at.\% Co implanted $\mathrm{ZnO}$ single crystal as secondary phase. All Raman spectra do not show the formation of any other secondary phases.

The four indicated surface regions are not randomly distributed, on the right hand side of figure 5.18 a scheme of the surface arrangement of those four surface regions is illustrated. The scheme presents to a greater or lesser extent continuous evolution from the (A) type surface to the (D) type surface from one side of the sample to the other. The inhomogeneous surface of the 16 at.\% Co implanted $\mathrm{ZnO}$ can be either induced by an inhomogeneous implantation or by a temperature gradient during the annealing or cooling down of the sample.

Figure 5.19 shows the XRD spectra of the 16 at.\% Co implanted $\mathrm{ZnO}$ single crystal after annealing at $700{ }^{\circ} \mathrm{C}$ and $900{ }^{\circ} \mathrm{C}$. After annealing at $700{ }^{\circ} \mathrm{C}$ two new diffraction peaks are observed in the XRD spectra of all Co implanted $\mathrm{ZnO}$ single crystals with Co concentrations of $\geq 8$ at.\%. However, those two peaks can not be assigned to any diffraction peak of $\mathrm{ZnCo}_{2} \mathrm{O}_{4}$, but these peaks fit very well to metallic hcp Zn and metallic Co (with hcp or cubic symmetry). A clear identification of these two phases could not been made, as no further diffraction of higher-order is observed in the XRD spectra. The diffraction at $43.4 \mathrm{deg}$. can be assigned to the (1011) Bragg peak of hcp Zn at $43.23 \mathrm{deg}$. [275]. On the other hand, the diffraction at $44.3 \mathrm{deg}$. can be assigned to the (111) Bragg peak of cubic Co (at 44.22 deg.) or to the (0002) Bragg peak of hcp Co (at 44.76 deg.) [275]. The second-order diffraction of all these Bragg peaks lies far outside of the measurement range of the used XRD diffractometer. Though, the usual crystal structure of metallic Co is the hexagonal crystal symmetry (hcp Co) and was observed in Co alloyed ZnO by synchrotron XRD as well as by Raman scattering $[14 ; 15 ; 341]$. The Raman signals of both hcp phases lie outside of the accessible region of the Raman setup [341].

After annealing at $900{ }^{\circ} \mathrm{C}$ the XRD spectrum of the 16 at.\% Co implanted $\mathrm{ZnO}$ single crystal changes completly. Both metallic phases could not be observed any more. However, the formation of oxide phases is favoured for annealing in oxygen rich ambients $[324 ; 342]$, resulting in the decomposition of the metallic phases to oxide phases. Five new diffraction lines can be found after annealing at $900{ }^{\circ} \mathrm{C}$ at: 28.28 deg., 36.4 deg., 42.1 deg., 51.1 deg., and 77.5 deg.. These diffraction peaks can be assigned to either $\mathrm{CoO}, \mathrm{Co}_{2} \mathrm{O}_{3}$ or $\mathrm{ZnCo}_{2} \mathrm{O}_{4}$ [275]. The diffraction peaks at 


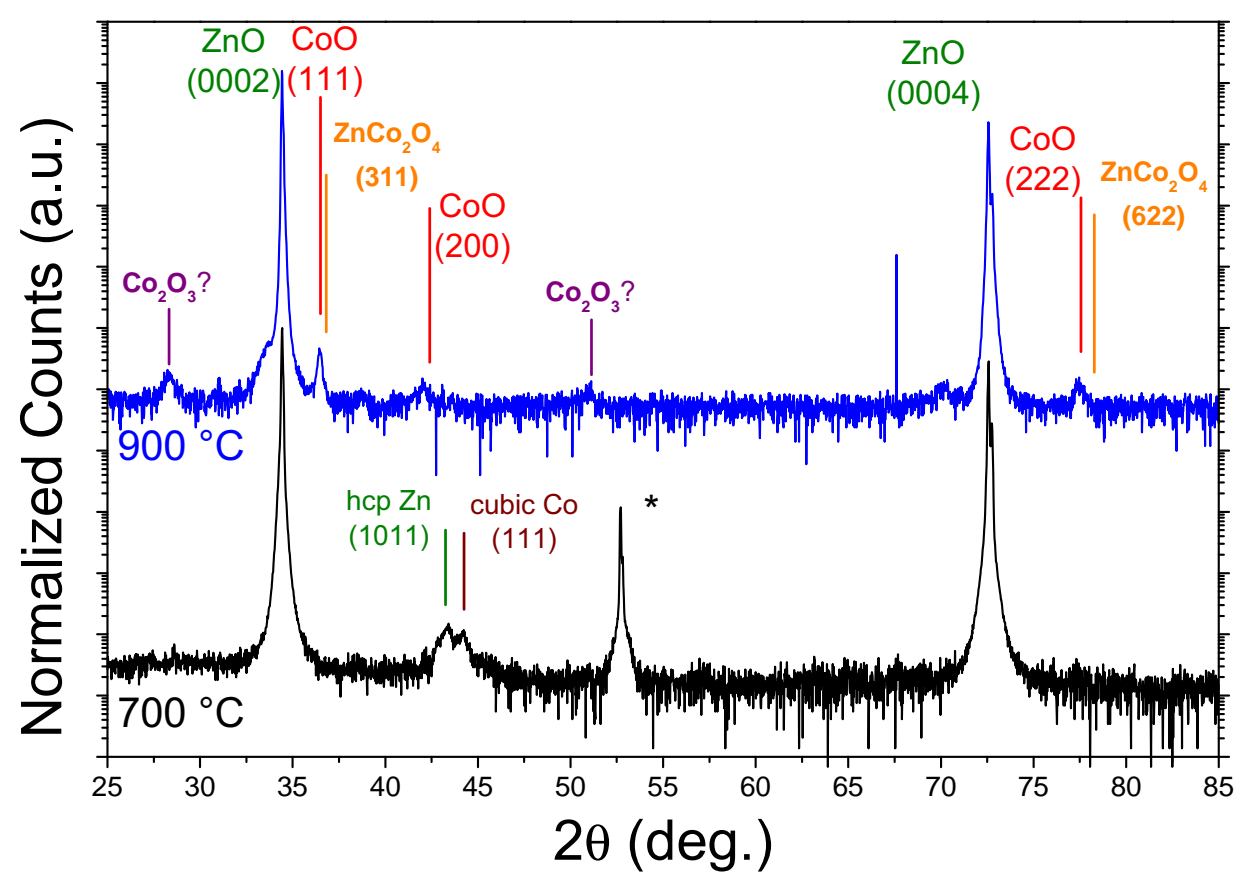

Figure 5.19: XRD diffractogram of the 16 at.\% Co implanted ZnO after annealing at $700^{\circ} \mathrm{C}$ and $900^{\circ} \mathrm{C}$. Besides the 0002 and 0004 peaks of the $\mathrm{ZnO}$ host, two additional features can be observed after annealing at $700^{\circ} \mathrm{C}$, which can be assigned to the (1011) peak of elemental hcp Zn and the (0002) peak of elemental hcp Co. After annealing at $900^{\circ} \mathrm{C}$ the situation changes completly, the additional peaks can be assigned to either $\mathrm{CoO}$ or $\mathrm{ZnCo}_{2} \mathrm{O}_{4}$, and $\mathrm{Co}_{2} \mathrm{O}_{3}$.

36.4 deg. and 77.5 deg. can be assigned to the (111) and (222) Bragg peaks of CoO or to the (311) and (622) peaks of $\mathrm{ZnCo}_{2} \mathrm{O}_{4}$ [275], whereby the position of the diffractions fit better to the $\mathrm{CoO}$ phase. The diffraction at $42.1 \mathrm{deg}$. fits perfectly to the (200) Bragg peak of CoO. The diffractions at 28.28 deg. and $51.1 \mathrm{deg}$. correspond to diffractions of $\mathrm{Co}_{2} \mathrm{O}_{3}$, however, the corresponding lattice planes are not specified in the available database [275]. Therefore, the assignement of those two diffraction lines to $\mathrm{CO}_{2} \mathrm{O}_{3}$ is ambiguous.

Figure 5.20 shows a high resolution micrograph of one large Co rich cluster in the 16 at.\% Co implanted $\mathrm{ZnO}$ single crystal. It is clearly visible that the cluster is composed of several different small Co rich grains, whereby the lattice directions of those grains are not justified to each other. This unjustified alignement of those grains support the observations of X-ray diffraction measurements after annealing at $900{ }^{\circ} \mathrm{C}$. If these grains would be aligned in the $\mathrm{ZnO}$ matrix, only the diffraction of one lattice plane group should be visible in figure 5.19. But the occurence of 


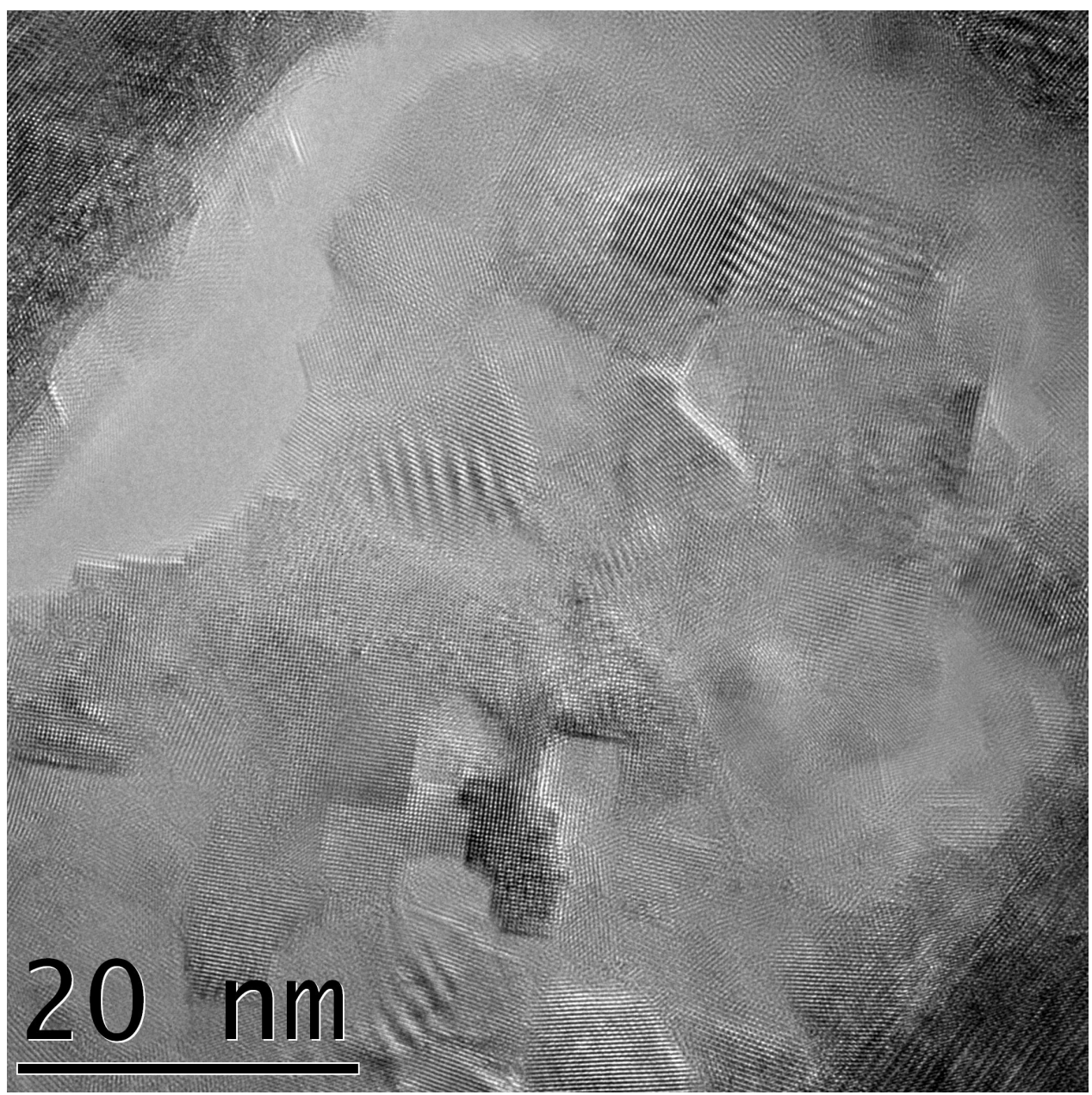

Figure 5.20: High resolution TEM micrograph of a Co rich cluster in the 16 at.\% Co implanted $\mathrm{ZnO}$ single crystal after annealing at $900^{\circ} \mathrm{C}$ shows several different non-oriented Co rich phases.

the (111) and (200) Bragg peak of CoO reveals this unjustified arrangement of the grains. However, an identification of a specific phase is not ambiguous as a FFT taken from one grain shows several superimposed diffraction patterns (not shown). A clear assignment of lattice directions in those FFTs is not possible. In the top right part of figure 5.20 the Co rich cluster seems to be partly amorphous, whereby in the top left part parts of the Co rich cluster are burned away after some seconds of exposure with the electron beam.

The data obtained by Raman and XRD show that at least three different phases exist after annealing at $700{ }^{\circ} \mathrm{C}$ : metallic $\mathrm{Zn}$, metallic $\mathrm{Co}$ and $\mathrm{ZnCo}_{2} \mathrm{O}_{4}$. During annealing at $900{ }^{\circ} \mathrm{C}$ both metallic phases oxidize to $\mathrm{CoO}, \mathrm{Co}_{2} \mathrm{O}_{3}$ and $\mathrm{ZnCo}_{2} \mathrm{O}_{4}$. 
However, HR-TEM analysis of those Co rich clusters suggest the occurrence of an amorphous Co rich phase after annealing at $900{ }^{\circ} \mathrm{C}$.

\subsection{3 $\mathrm{ZnFe}_{2} \mathrm{O}_{4}$ in Fe implanted $\mathrm{ZnO}$}

For all Fe implanted $\mathrm{ZnO}$ single crystals with Fe concentrations $\geq 8$ at.\% Raman spectra reveal the formation of secondary phases. Figure 5.21 shows the Raman spectra of the 16 at.\% Fe implanted $\mathrm{ZnO}$ sample after annealing at $700{ }^{\circ} \mathrm{C}$ on different sample points. Small particles peel off from the annealed sample surface, therefore three different surface types can be found under the optical light microscope: an intact surface region, an partly peeled-off surface region, and a completly peeled-off surface region (see figure 5.21). The Raman spectra from these three regions differ from each other. The spectra from the intact and partly peeled-off surface region show an additional phonon band between $500 \mathrm{~cm}^{-1}$ and $700 \mathrm{~cm}^{-1}$ (see figure 5.21), which has an absolutly different shape than the disorder induced $A_{1}(L O)$ phonon band in low dose transition metal implanted $\mathrm{ZnO}$ single crystals. This additional band is unstructured and therefore it is difficult to assign unambiguously a secondary phase. However, EDS line scans show a comparable behavior like the line scan of the 16 at.\% Mn implanted $\mathrm{ZnO}$ single crystal shown in figure 5.14. Therefore, one can assume, that this band may occur because of Fe rich segregations within the $\mathrm{ZnO}$ matrix. Though, nearly all possible iron oxide phases as well as zinc ferrite $\left(\mathrm{ZnFe}_{2} \mathrm{O}_{4}\right)$ show strong phonon modes within the spectral region of this band [343-346].

The completely peeled-off surface region exhibits a Raman spectrum (see figure 5.21), which shows no difference to the Raman spectrum of pure ZnO. On the other hand the Raman spectrum of an peeled-off sample piece (see inset in figure 5.21) shows the additional broad band between $500 \mathrm{~cm}^{-1}$ and $700 \mathrm{~cm}^{-1}$. This observation suggests, that the destruction of the sample surface is effected by the formation of the segregations, the lattice mismatch and the size of those segregations could induce stress. The segregation induced stress leads to the peel-off of the implanted layer.

Figure 5.22 shows the X-ray diffraction spectra of the 16 at.\% Fe implanted ZnO single crystal after annealing at $700{ }^{\circ} \mathrm{C}$ and $900{ }^{\circ} \mathrm{C}$. Three additional diffraction peaks can be observed at: 36.9 deg., 56.9 deg., and 78.8 deg., whereby after annealing $700{ }^{\circ} \mathrm{C}$ only the diffraction at 56.9 deg. can be surely identified. For samples with lower Fe concentration no additional diffraction peak can be surely identified after annealing at $700{ }^{\circ} \mathrm{C}$. All three peaks fit very well to the Bragg peaks of zinc ferrite 


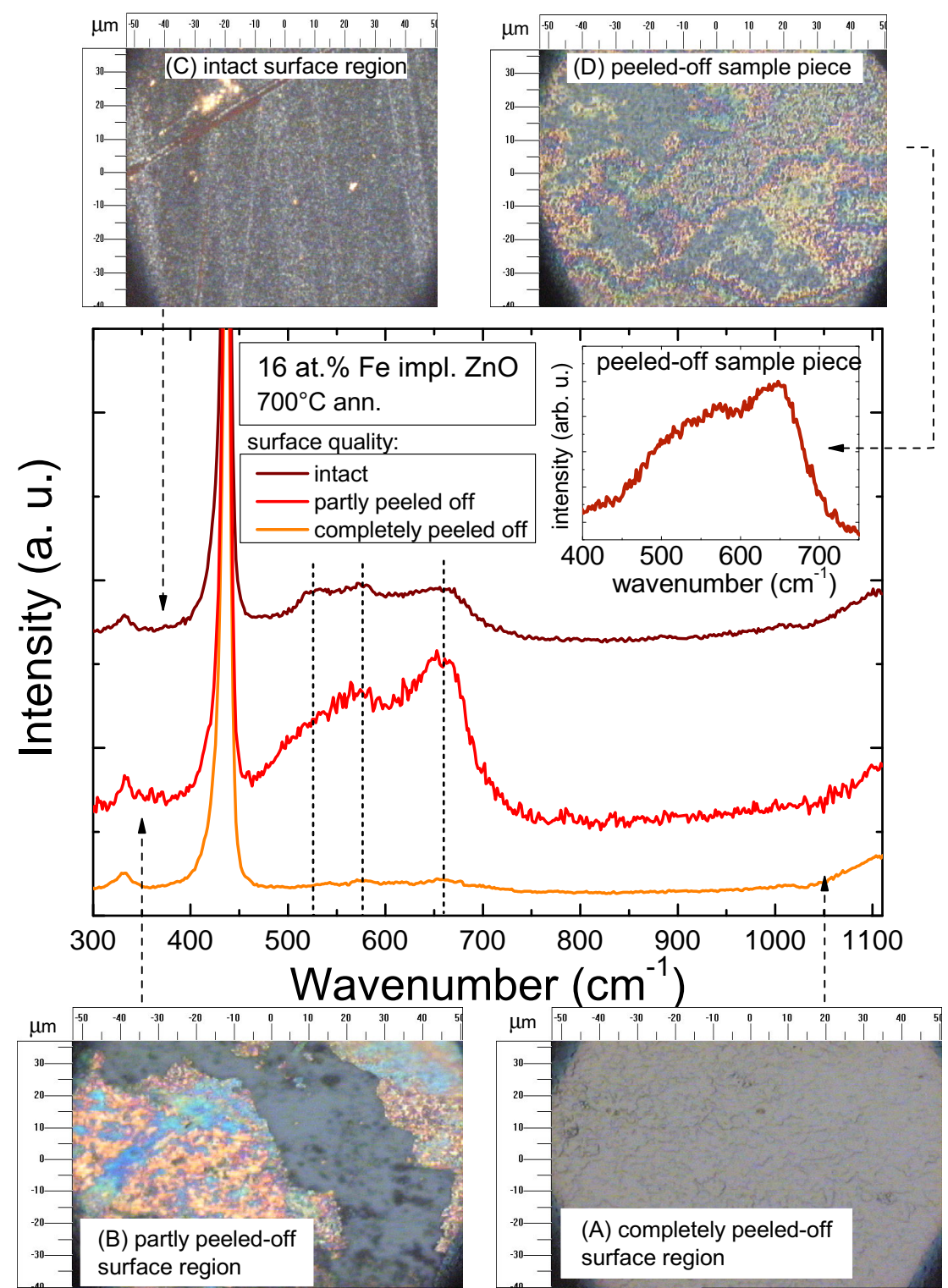

Figure 5.21: Raman spectra of different spots on 16 at.\% Fe implanted $\mathrm{ZnO}$ single crystal after $700^{\circ} \mathrm{C}$ annealing [19]. The optical microscope pictures show the studied surface regions. Spectra from bottom to top: laser focused on (A) completely peeled-off surface region, (B) partly peeled-off surface region, (C) intact surface region, and (D) peeled-off sample flake (inset).

$\left(\mathrm{ZnFe}_{2} \mathrm{O}_{4}\right)$, whereby the diffraction peak at $56.9 \mathrm{deg}$. can either be assigned to the (511) or (333) diffraction. With the Scherrer relation given in section 4.2 the size of the segregations is estimated to $(11.6 \pm 1.2) \mathrm{nm}$ after annealing at $700{ }^{\circ} \mathrm{C}$ and $(10 \pm 0.5) \mathrm{nm}$ after annealing at $900{ }^{\circ} \mathrm{C}$. The evaluation of the crystallite size of the segregations exhibits a decrease with increasing annealing temperature, however, 


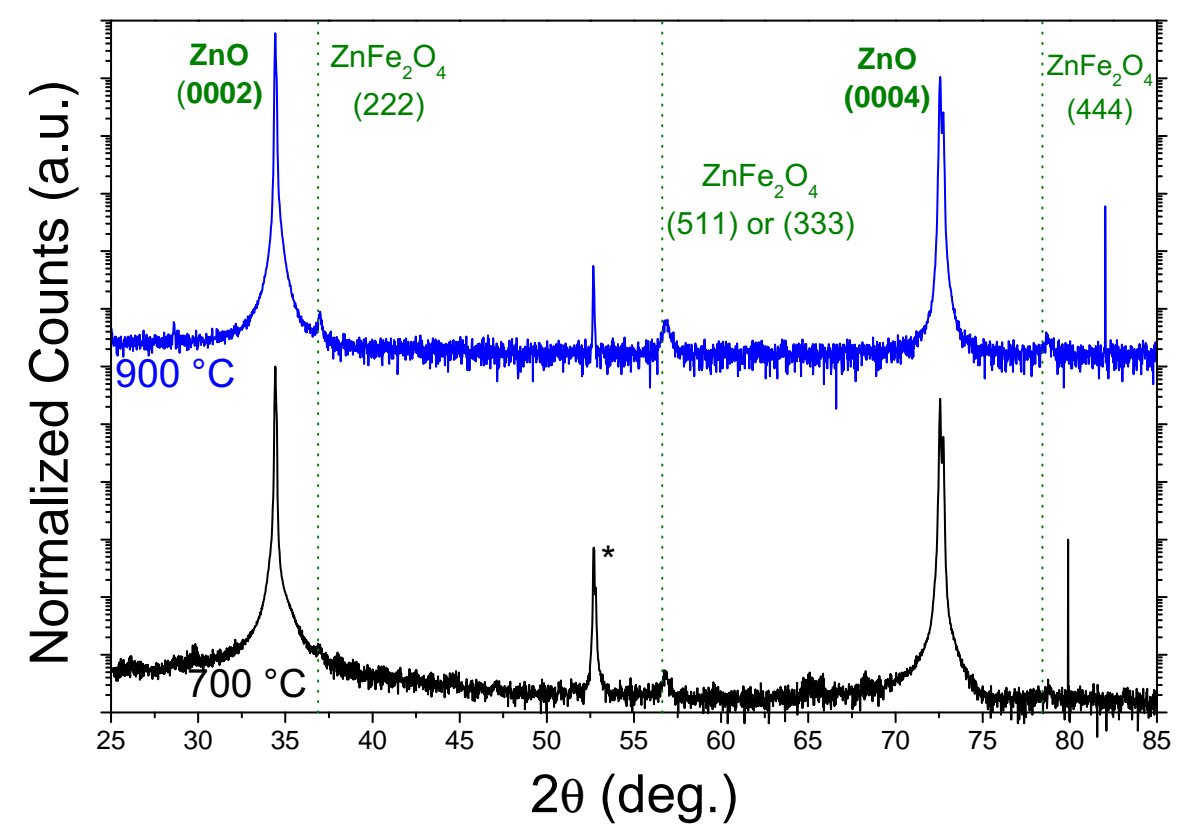

Figure 5.22: XRD diffractogram of 16 at.\% Fe implanted ZnO single crystal after annealing at $700^{\circ} \mathrm{C}$ and $900^{\circ} \mathrm{C}$. Besides the (0002) and (0004) peaks of the $\mathrm{ZnO}$ host, two additional features can be observed. The additional feature is assigned to the (511) diffraction peak of $\mathrm{ZnFe}_{2} \mathrm{O}_{4}$.

taking the error values into account no real size difference can be observed. Though, the weak increase of the $\mathrm{ZnFe}_{2} \mathrm{O}_{4}$ can be explained by a decomposition process of other iron oxide phases into zinc ferrite during the annealing at $900{ }^{\circ} \mathrm{C}$. The formation of different iron rich phases and the decomposition into zinc ferrite was reported before by Zhou et al. [16; 17]. The volume ratio of all other iron oxides might be too small to detect their diffraction peaks with a conventional XRD setup. Zhou et al. had oberserved a parallel alignement between the zinc ferrite [111] axis and the c-axis of $\mathrm{ZnO}$. If this statement is truth, then the diffraction at $56.9 \mathrm{deg}$. corresponds to the (333) Bragg peak of zinc ferrite.

Figure 5.23 shows the high resolution TEM micrograph of the 16 at.\% Fe implanted $\mathrm{ZnO}$ single crystal after annealing at $900{ }^{\circ} \mathrm{C}$. The Fe rich phase and the $\mathrm{ZnO}$ phase are indicated in figure 5.23, whereby it is necessary to note that the 'ZnO' labelled domain is not pure $\mathrm{ZnO}$. The label ' $\mathrm{ZnO}$ ' is chosen to avoid confusion, this domain is the Fe poor region. Within the figure the FFTs of both domains are shown. The FFT of the Fe rich phase is consistent with an electron diffraction pattern of a cubic lattice arrangement with a [11̄] zone axis [294]. The distances of the marked diffraction spots to the central spot in the Fe rich FFT correspond to 


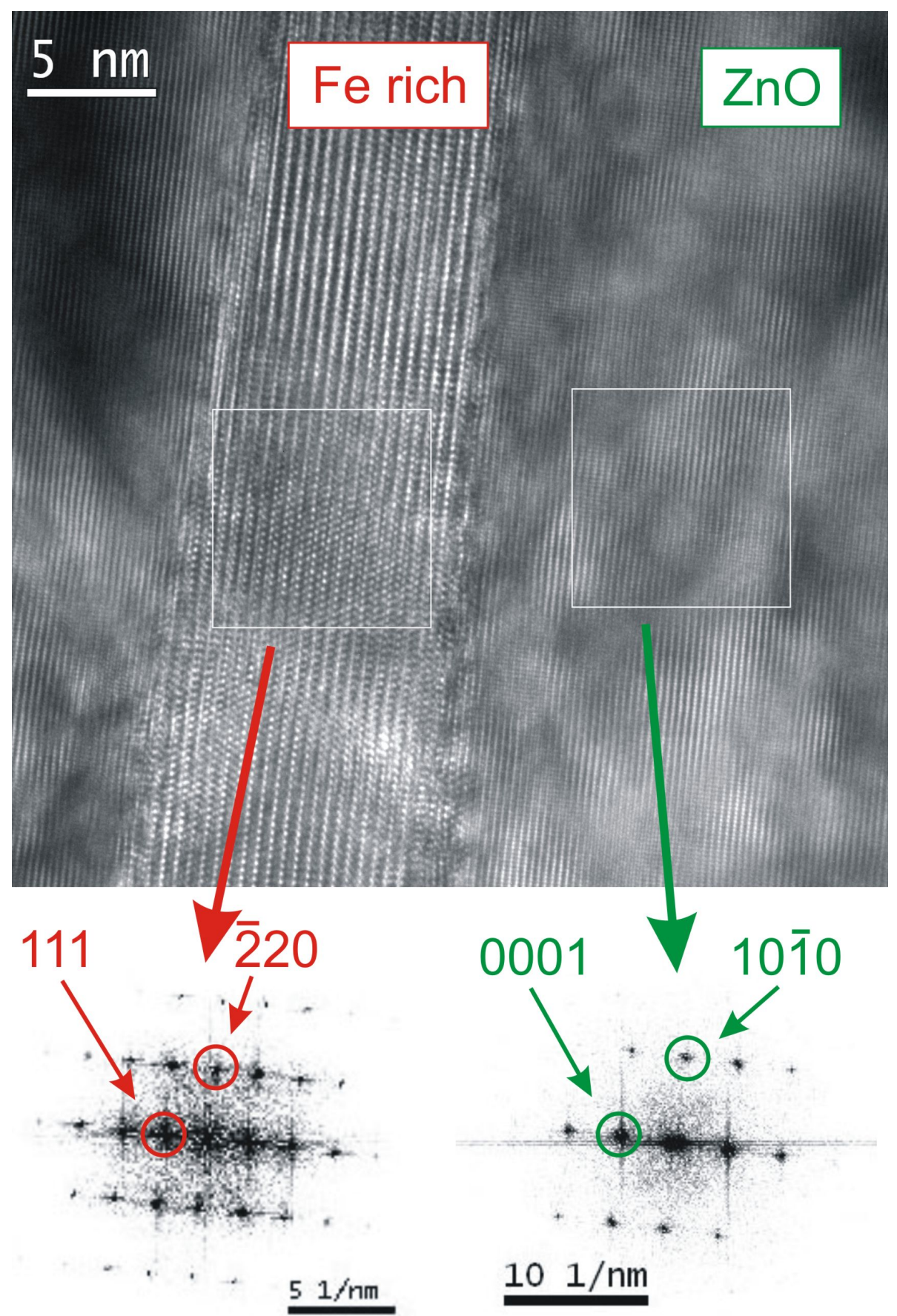

Figure 5.23: The high resolution TEM micrograph presents a Fe rich segregation in the 16 at.\% Fe implanted ZnO after annealing at $900{ }^{\circ} \mathrm{C}$. The FFT of the highlighted region in $\mathrm{ZnO}$ is shown on the right hand side and on the left hand side the FFT of the Fe rich segregation. 
the lattice spacing of the (111) and (220) lattice planes of $\mathrm{ZnFe}_{2} \mathrm{O}_{4}$. A comparision of the lattice directions via the FFTs in figure 5.23 reveal a parallel arrangement of the [0001] axis of $\mathrm{ZnO}$ and [111] direction of $\mathrm{ZnFe}_{2} \mathrm{O}_{4}$, and a parallel arrangement of the $[10 \overline{1} 0] \mathrm{ZnO}$ lattice direction with the [220] lattice direction of $\mathrm{ZnFe}_{2} \mathrm{O}_{4}$.

\subsubsection{Formation of $\mathrm{NiO}$ secondary phases}

The Raman spectra of low dose $\mathrm{Ni}$ implanted $\mathrm{ZnO}$ single crystals after annealing at $700{ }^{\circ} \mathrm{C}$ are presented in figure $5.6 \mathrm{c}$, which shows only the $E_{2}^{\text {high }}$ and the disorder induced $A_{1}(L O)$ phonon band. The intensity of the disorder induced $A_{1}(L O)$ phonon band scales with the $\mathrm{Ni}$ ion implantation fluence and shows an increase with increasing fluence; however, this observation does not change for the high dose $\mathrm{Ni}$ implanted $\mathrm{ZnO}$ samples. The Raman spectra (not shown) of high dose Ni implanted $\mathrm{ZnO}$ single crystals show no further signal, which could correspond to a secondary phase. The absence of any additional Raman mode especially in the 16 at.\% Ni implanted sample is not expected, due to the observaltion of secondary phase Raman modes in all other transition metal implanted $\mathrm{ZnO}$ single crystals.

Figure 5.24 shows the X-ray diffraction spectra of the 16 at.\% Ni implanted $\mathrm{ZnO}$ single crystal after annealing at $700{ }^{\circ} \mathrm{C}$ and $900{ }^{\circ} \mathrm{C}$. Three additional diffractions can be observed at about: 37.1 deg., 44.4 deg., and 79.1 deg.. These three diffractions were observed in all $\mathrm{Ni}$ implanted $\mathrm{ZnO}$ single crystals with Ni concentrations $\geq 8$ at. $\%$ after annealing at $700{ }^{\circ} \mathrm{C}$ (see figure 5.13). The diffraction peak at 44.4 deg. dissapears during annealing at $900{ }^{\circ} \mathrm{C}$, suggesting a transformation of the corresponding phase. This diffraction peak can be assigned to the 111 Bragg peak of cubic Ni at $44.51 \mathrm{deg}$. or to the 011 Bragg peak of hcp Ni [275]. The annealing in oxygen ambient at higher temperatures would oxidize the metallic Ni segregations to $\mathrm{NiO}$. The 111 and 222 Bragg peaks of $\mathrm{NiO}$ at $37.25 \mathrm{deg}$. and $79.41 \mathrm{deg}$. fit very well to the observed diffraction peaks at $37.1 \mathrm{deg}$. and 79.1 deg.. However, elemental Ni crystallizes in the cubic form under ambient conditions and the formation of cubic $\mathrm{Ni}$ segregations was observed in $\mathrm{Ni}$ alloyed $\mathrm{ZnO}[15 ; 18]$. After $700{ }^{\circ} \mathrm{C}$ annealing the crystallite sizes of the $\mathrm{NiO}$ and metallic $\mathrm{Ni}$ is estimated to $(9.2 \pm 0.2) \mathrm{nm}$ and $(5.1 \pm 0.1) \mathrm{nm}$, respectively. The crystallite size of $\mathrm{NiO}$ increases to $(17.5 \pm 0.2) \mathrm{nm}$, which supports on one hand the oxidization of metallic Ni and on the other hand the Ostwald ripening can also discribe the increase of the $\mathrm{NiO}$ grains. Both measured diffraction angles differ marginally from the Bragg positions of $\mathrm{NiO}$ given in the database [275] and reveal a tensile strain affecting the lattice constant of the $\mathrm{NiO}$ 


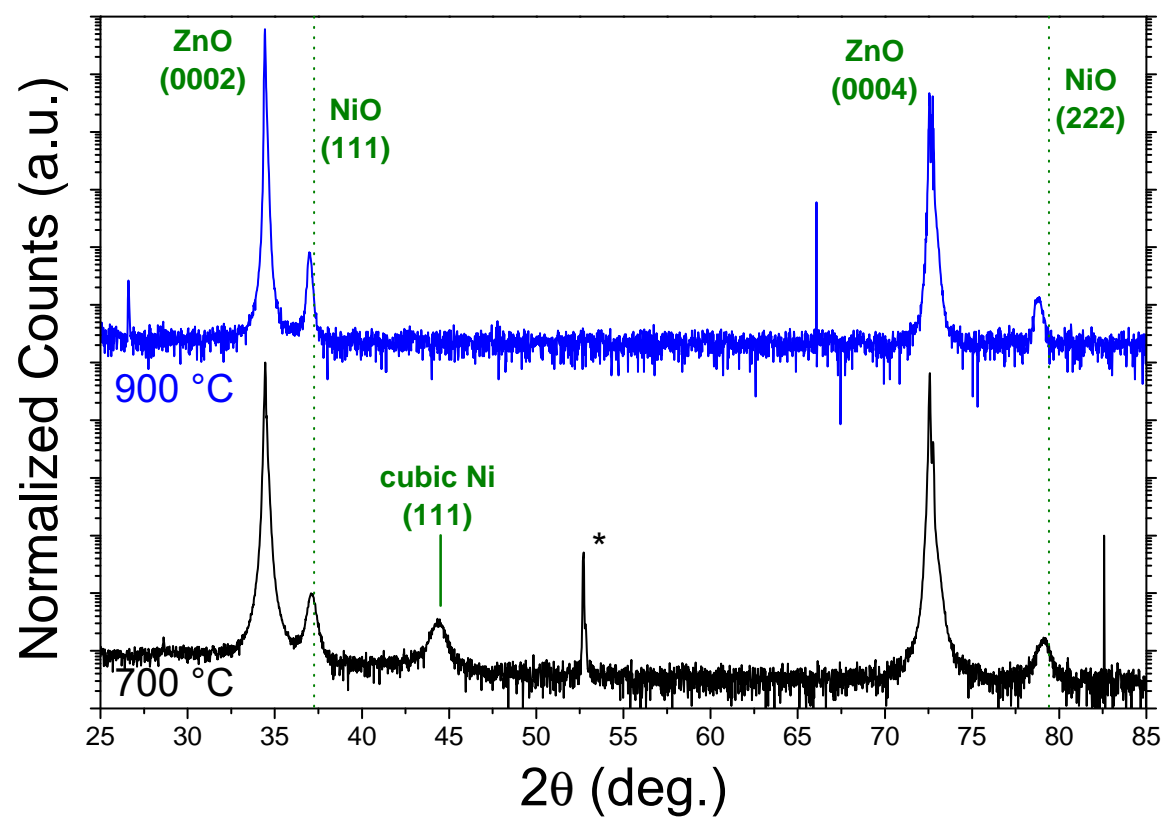

Figure 5.24: XRD diffractogram of 16 at.\% Ni implanted $Z n O$ single crystal after annealing at $700^{\circ} \mathrm{C}$ and $900^{\circ} \mathrm{C}$. Besides the (0002) and (0004) peaks of the $\mathrm{ZnO}$ host, four additional features can be observed. The additional features correspond to the (111) and (222) peaks of NiO and the (111) peak of elemental cubic Ni, which decomposes during the annealing at $900{ }^{\circ} \mathrm{C}$.

grains. The XRD spectra in figure 5.24 suggest an parallel arrangement of the $\mathrm{ZnO}$ c-axis and the (111) direction of $\mathrm{NiO}$.

TEM analysis of the 16 at.\% $\mathrm{Ni}$ implanted $\mathrm{ZnO}$ sample after annealing show two different domains. Figure 5.25 shows the high resolution TEM micrograph of a boundary area between these two different domains (the $\mathrm{NiO}$ domain and $\mathrm{ZnO}$ domain are indicated). The two boxes in figure 5.25 indicate the areas from which the FFTs of the corresponding domains are taken. Both FFTs indicate different lattice arrangements, whereby the left hand side FFT indicates the $\mathrm{ZnO}$ domain. It is necessary to note that the ' $\mathrm{ZnO}$ ' labelled domain is not pure $\mathrm{ZnO}$. The label ' $\mathrm{ZnO}$ ' is chosen to avoid confusion, this domain is the Ni poor region. The FFT labelled with $\mathrm{NiO}$ is consistent with a cubic lattice arrangement with a [110] zone axis (see figure 5.25) [294]. The distances of the marked diffraction spots to the central spot in the $\mathrm{NiO}$ labelled FFT correspond to the lattice spacing of the (111) and (003) lattice planes of $\mathrm{NiO}$. A comparision of the lattice directions via the FFTs in figure 5.25 reveals a parallel arrangeent of the $\mathrm{ZnO}$ c-axis and [111] lattice direction of 

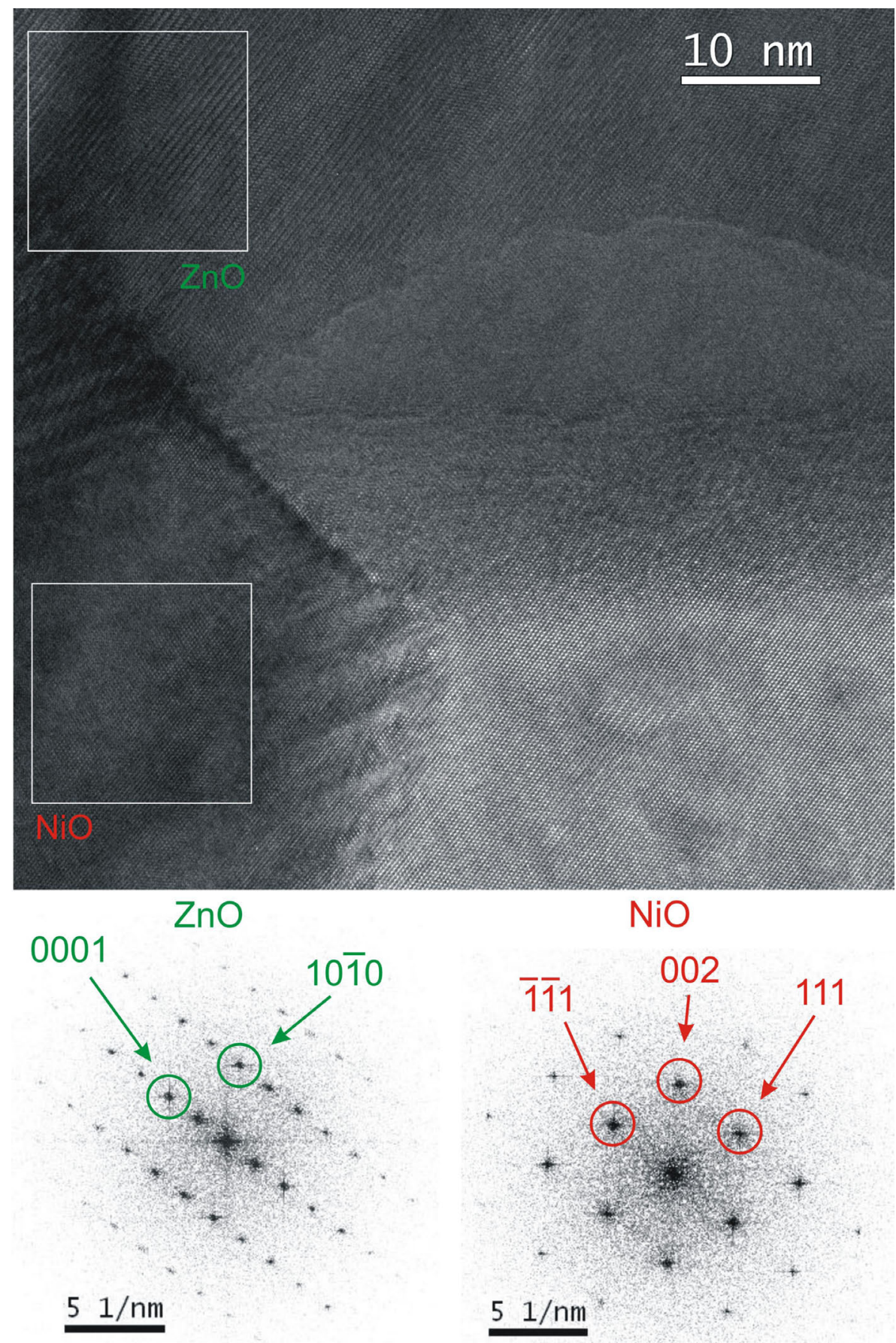

Figure 5.25: The high resolution TEM micrograph presents the boundary area between a Ni rich segregation (indicated as $\mathrm{NiO}$ ), Ni implanted $\mathrm{ZnO}$ (indicated as $\mathrm{ZnO}$ ) and a bright domain in the (down right) in the 16 at.\% Ni implanted $\mathrm{ZnO}$ after annealing at $900^{\circ} \mathrm{C}$. The FFT of the NiO segregation is shown on the right hand side and the FFT of the ZnO phases on the left hand side. 
$\mathrm{NiO}$, and a parallel arrangement of the [1010] ZnO lattice direction with the [113] lattice direction of $\mathrm{NiO}$.

\subsection{Summary}

In this chapter the structural impact of transition metal ion implantation into $\mathrm{ZnO}$ single crystals and $\mathrm{ZnO}$ nanowires was studied. For low transition metal concentrations $(\leq 4$ at.\%) only a damage formation due to the ion implantation generated defects can be observed. The formation of defects was observed by an increase of the $A_{1}(L O)$ phonon band intensity in Raman spectra, whereby the intensity scales with the transition metal ion implantation fluence. Annealing in air leads to a recovery of the lattice and a deacrease of the $A_{1}(L O)$ phonon band, but the initial state was not reached for all transition metal implanted $\mathrm{ZnO}$ single crystals. This situation was also observed in all TEM micrographs, which reveal a high density of defects and indicates a too low annealing temperature for a complete recovery. However, the annealing behavior, which was observed for transiton metal implanted $\mathrm{ZnO}$ single crystals, does not show any difference for transition metal implanted $\mathrm{ZnO}$ nanowires.

With energy electron loss spectroscopy and energy dispersive X-ray spectroscopy it was shown, that the incorporation of transition metals was succesful. No outdiffusion of the implanted transition metals was observed after annealing at $700{ }^{\circ} \mathrm{C}$. Therefore, the ion implantation of transition metals is a technique for the incorporation of transition metals into $\mathrm{ZnO}$ nanowires even beyond solubility limits.

With the exception of Mn, no additional phonon modes in the Raman spectra of transition metal implanted $\mathrm{ZnO}$ single crystals were found, which could be assigned to local vibration modes of the corresponding transition metal. Mn implanted $\mathrm{ZnO}$ single crystals had shown an additional phonon mode at $519 \mathrm{~cm}^{-1}$, which is a candidate for a local vibration mode of $\mathrm{Mn}$ ions on a zinc lattice side.

High dose transition metal implanted $\mathrm{ZnO}$ single crystals had shown additional features in Raman and XRD spectroscopy. These additional features were assigned to secondary phase segregations in the $\mathrm{ZnO}$ matrix. The formation of secondary phases after annealing at $700{ }^{\circ} \mathrm{C}$ was observed for all transition metal implanted $\mathrm{ZnO}$ single crystals with transition metal concentrations of $\geq 8$ at.\%. The detection limits of a conventional XRD setup and Raman differ slightly. A clear identification of the chemical nature of a segregation by only one technique is not possible. At least the combination of Raman, XRD and TEM is necessary to delimitate the chemical nature of the segregations. Commonly XRD measurements were used to destinguish 
the formation of secondary phases, but the verification of secondary phases with XRD is not easy. All XRD measurements of transition metal implanted ZnO single crystals presented in this thesis need a very good alignement of the single crystals in the X-ray beam. If the alignement was not perfect, the maximum intensity of the $\mathrm{ZnO}$ diffraction is several orders of magnitude lower, resulting also in a reduction of the intensity of the diffractions of segregations. On the other hand, the verification of segregations is only possible for Raman active materials; non-active materials such as $\mathrm{NiO}, \mathrm{CoO}$ or $\mathrm{MnO}_{2}$ are not detectable with Raman.

Annealing at higher temperatures of $900{ }^{\circ} \mathrm{C}$ results in a phase transformation of the segregations in $\mathrm{Ni}, \mathrm{Mn}$ and Co implanted $\mathrm{ZnO}$ single crystals. No clear indications were observed for a phase transformation of Fe rich segregations in the Fe implanted $\mathrm{ZnO}$ single crystals.

Table 5.2 presents a list of possible or identified secondary phases in transition metal alloyed $\mathrm{ZnO}$ and their bulk magnetic properties. Potzger et al. and Zhou et al. had shown that the formation of segregations with diameters of some nanometers can change the magnetic nature of the segregations in $\mathrm{ZnO}$ [14-18]. The authors

Table 5.2: Magnetic Properties of potential or identified second phases (discussion for nanoparticels see text).

\begin{tabular}{llr}
\hline Phase & Nature of magnetism & Reference \\
\hline $\mathrm{Mn}$ & Anti-ferromagnetic & {$[12]$} \\
$\mathrm{MnO}$ & Anti-ferromagnetic & {$[12 ; 91 ; 347]$} \\
$\mathrm{MnO}_{2}$ & Anti-ferromagnetic & {$[12 ; 347]$} \\
$\mathrm{Mn}_{3} \mathrm{O}_{4}$ & Ferromagnetic & {$[12]$} \\
$\mathrm{ZnMn}_{2} \mathrm{O}_{4}$ & Ferromagnetic & {$[12]$} \\
$\mathrm{Co}$ & Ferromagnetic & {$[91]$} \\
$\mathrm{CoO}$ & Anti-ferromagnetic & {$[91 ; 137 ; 347]$} \\
$\mathrm{Co}_{3} \mathrm{O}_{4}$ & Anti-ferromagnetic & {$[137]$} \\
$\mathrm{ZnCo}_{2} \mathrm{O}_{4}$ & Paramagnetic & {$[348]$} \\
$\mathrm{Fe}$ & Ferromagnetic & {$[91]$} \\
$\mathrm{Fe}_{3} \mathrm{O}_{4}$ & Ferrimagnetic & {$[345]$} \\
$\mathrm{ZnFe}_{2} \mathrm{O}_{4}$ & Anti-ferromagnetic & {$[16]$} \\
$\mathrm{Ni}$ & Ferromagnetic & {$[91]$} \\
$\mathrm{NiO}$ & Anti-ferromagnetic & {$[91 ; 347]$} \\
\hline
\end{tabular}


had shown that the size of the segregations play a major role, due to changes of the magnetic nature from the anti-ferromagnetic or ferromagnetic state to a superparamagnetic state [14-18]. It was shown that the transition temperature of nanosized clusters between the superparamagnetic state and the paramagnetic state depends on the size of the segregations $[16 ; 17]$ and could be the explanation for the extreme variance of the experimental determined Curie temperature of transition metal alloyed $\mathrm{ZnO}[6 ; 11]$. 


\section{Chapter 6}

\section{Luminescence of transition metal implanted $\mathrm{ZnO}$}

$\mathrm{ZnO}$ is a typically n-type doped wide band gap semiconductor with numerous intrinsic and extrinsic donors $[63 ; 97 ; 98]$. Most of these donors are optical active and dominate the luminescence of $\mathrm{ZnO}$ at low temperature $[73 ; 107 ; 349]$. Figure 6.1 shows a survey of the $\mathrm{ZnO}$ luminescence at low temperature for nanowires (a) and single crystals from CrysTec (b). Typically, luminescence spectra of $\mathrm{ZnO}$ are divided into two fragments: near band gap luminescence in the ultra-violet (UV) range and the luminescence of deep centers in the visible/near infrared range.

All luminescence spectra, plotted in figure 6.1 on logarithmic scale, show a broad and faint luminescence band in the visible range, which is labeled as 'green luminescence band'. This band has two different shapes as visible in figure 6.1: a structured or a Gaussian like. Additionally to the luminescence of virgin samples, the circumstances after annealing in air at $700{ }^{\circ} \mathrm{C}$ is shown for both samples in figure 6.1. The intensity of the structured green luminescence band in figure 6.1a increases slightly compared to the near band gap luminescence. The Gaussian like green luminescence band in figure $6.1 \mathrm{~b}$ shows a contrary behavior and decreases in intensity compared to the near band gap luminescence. The green luminescence band is typically assigned to oxygen vacancies $V_{O}[70 ; 71 ; 323 ; 350]$ or copper $[90 ; 351]$. Annealing in air or oxygen should result in a reduction of the oxygen vacancy concentration. Therefore, the intensity of this band should decrease [323; 350]. However, annealing in air does not change the concentration of copper impurities in $\mathrm{ZnO}$. The intensity of the green band should not show any effect due to the annealing in air, unless the nature of this defect is more complex. The nature of the green luminescence band is discussed in more detail in section 6.3. 

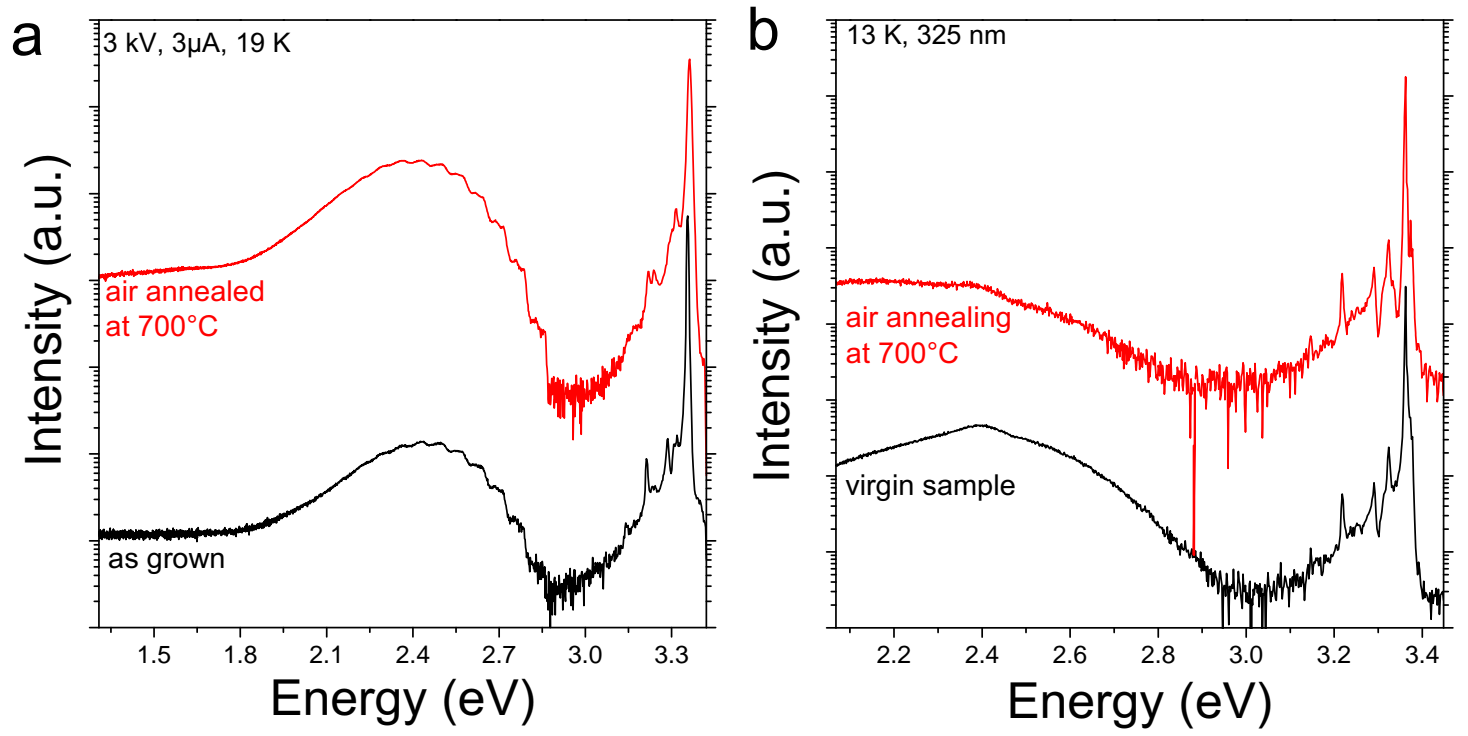

Figure 6.1: Survey of ZnO luminescence at low temperature for $Z n O$ nanowires (a) and CrysTec single crystals (b) show a sharp and intensive UV luminescence. The cathodoluminescence spectra of ZnO nanowires in (a) show a broad structured green luminescence band, whereas the photoluminescence spectra of Crys Tec single crystals show an unstructured green luminescence band.

Figure 6.2 shows a detailed look on the near band gap luminescence, which is a composition of sharp and intense transitions. Those transitions are assigned to free exciton transitions (FX) and bound exciton lines [73; 107]. Thereby, bound exciton transitions are divided into acceptor bound exciton transitions (AX) and donor bound exciton transitions (DX) $[73 ; 107]$. The near band gap luminescence of as-grown as well as annealed $\mathrm{ZnO}$ nanowires show several narrow lines with low differences in their spectral position (see figure $6.2 \mathrm{a}$ ). The $\mathrm{I}_{2-3 a}, \mathrm{I}_{6}$ and $\mathrm{I}_{9}$ lines dominate the spectra of as-grown as well as annealed $\mathrm{ZnO}$ nanowires, which are assigned to donor bound exciton transitions [73; 107]. The near band gap luminescence of Crys Tec $\mathrm{ZnO}$ single crystals are dominated by the $\mathrm{I}_{5-6}$ luminescence lines with a weak intensity of the $\mathrm{I}_{3,3 a}$ line (see figure $6.2 \mathrm{~b}$ ). The free exciton transitions FX can be observed as a weak shoulder on the high energy tail in all $\mathrm{ZnO}$ nanowires and as sharp and low intense transition line in the $\mathrm{ZnO}$ single crystals. The spectral positions of all possible donor bound exciton lines and free exciton transitions are listed in table $6.1[73-75 ; 107 ; 352]$. The chemical nature of this donor bound exciton transitions is up to now ambiguous with some exceptions. Extrinsic impurities were found to be the origin for the $\mathrm{I}_{1}, \mathrm{I}_{4}, \mathrm{I}_{6,6 a}, \mathrm{I}_{8}$ and $\mathrm{I}_{9}$ donor bound transitions (see table 6.1) [73-75]. The $\mathrm{I}_{6}$ and $\mathrm{I}_{9}$ transitions are assigned to $\mathrm{Al}$ and $\mathrm{In}[73 ; 75]$, 

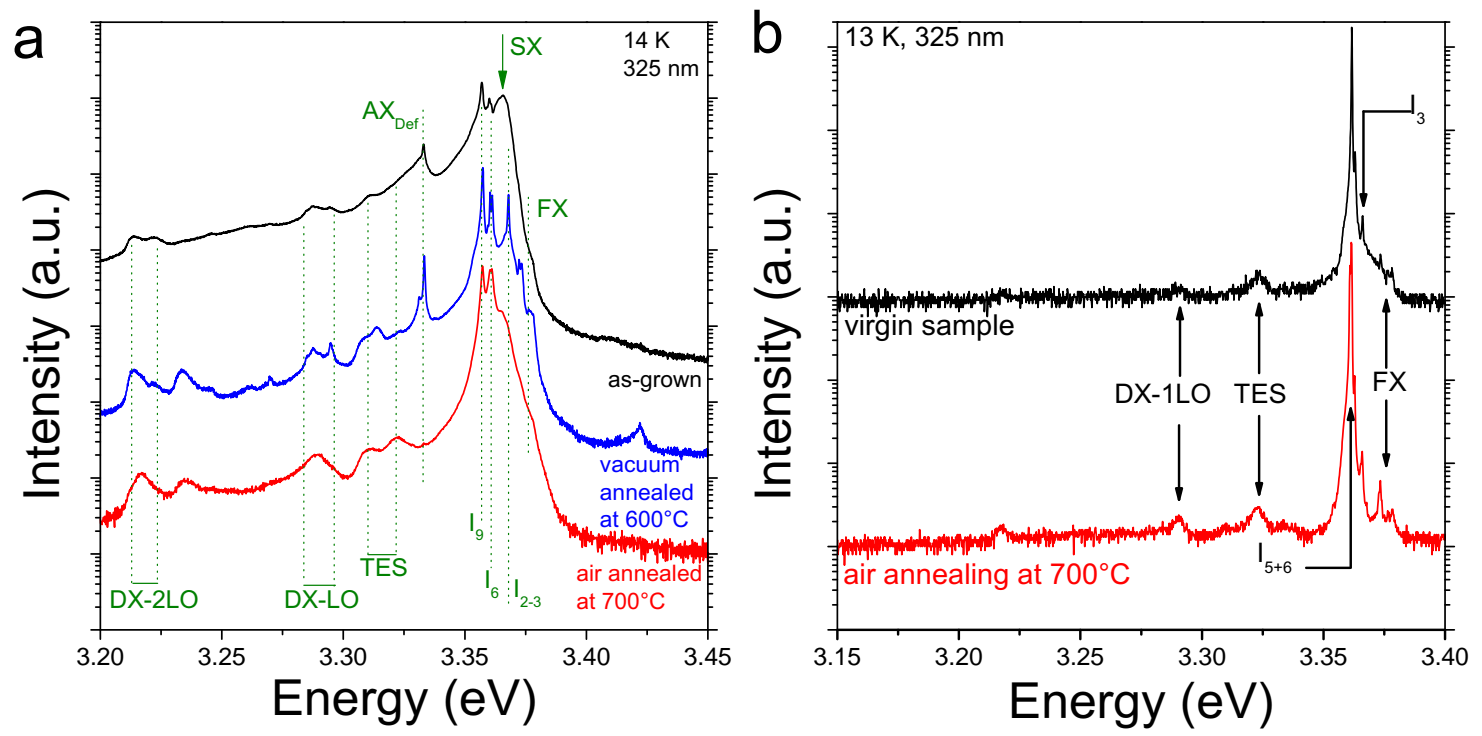

Figure 6.2: Ultra-violet photoluminescence of unimplanted and annealed $\mathrm{ZnO}$ samples at low temperatures excited with $325 \mathrm{~nm}$ line of the HeCd laser. (a) shows the luminescence of nanowire samples and (b) of single crystals from CrysTec.

respectively. The radioactive photoluminescence experiment to assign the $\mathrm{I}_{9}$ line to indium is presented in the next section 6.1.

However, also intrinsic defects could form a shallow donor, which could be the reason of a donor bound exciton line. Theoretical calculations show, that interstitial zinc $\mathrm{Zn}_{i}$ is a shallow donor [353; 354]. Sann et al. assigns this interstitial zinc as origin of the $\mathrm{I}_{3,3 a}$ lines of the near band gap luminescence of $\mathrm{ZnO}$ [352]. The appearance of the $\mathrm{I}_{3,3 a}$ lines in figure 6.2 confirms the zinc rich growth conditions especially for the $\mathrm{ZnO}$ nanowires (see section 3.3.1). The photoluminescence of CrysTec single crystals as well as of $\mathrm{ZnO}$ nanowires present marginal differences in figure 6.2. Especially the free exciton transitions show a slight increase. Annealing under high vacuum ambient of $\mathrm{ZnO}$ nanowires in $6.2 \mathrm{~b}$ reveal a narrowing and more defined donor bound luminescence than air annealed $\mathrm{ZnO}$ nanowires. However, the observed differences are within the tolerance between different samples, especially for different $\mathrm{ZnO}$ nanowire growth runs.

In figure 6.2a the transition at an energy of about $3.367 \mathrm{eV}$ is marked with SX. This transition is only observed in nanocrystalline $\mathrm{ZnO}$ and assigned to recombination of surface excitons [355; 356]. The SX peak intensity increases compared to the DX peak intensity with decreasing nanowire diameter [355]. Besides the SX transition of surface excitons, no further peak or band originated from the low size of the $\mathrm{ZnO}$ nanowires can be observed. 
Figure 6.2 indicates several luminescence transitions at lower energies than the donor bound exciton transitions. At $3.333 \mathrm{eV}$ a defined but weak transition occurs, which is labeled with $\mathrm{AX}_{\text {Def }}$ in figure 6.2. Analysis of single crystalline epitaxial grown $\mathrm{ZnO}$ films show this luminescence at crystalline defects and is therefore assigned to structural defects [73]. However, neither the type of defect nor the chemical nature contributing to this defect is up to now known. It should be noted that this spectral position fits very well with the spectral position of 'two electron satellites' (TES) of the donor bound exciton transitions [73; 357; 358]. Every TES transition is related to a defined donor bound exciton transition $\mathrm{I}_{x}$. During the recombination of a bound exciton, the neutral donor is excited from its $1 s$ ground state to an excited state such as $2 s, 2 p$, etc $[357 ; 359]$. Therefore, the energy difference between the TES

Table 6.1: List of free exciton (FX) and donor bound exciton (DX) lines and their chemical nature ( ${ }^{*}$ indicate ionized donor bound exciton transitions $\left(D^{-} X\right)$, data and chemical assignment are taken from [73-75; 10\%; 352]).

\begin{tabular}{lrrrr}
\hline line & \multicolumn{2}{c}{ spectral position } & $\begin{array}{r}\text { donor binding } \\
\text { energy } \\
(\mathrm{meV})\end{array}$ & $\begin{array}{r}\text { chemical } \\
\text { nature }\end{array}$ \\
\cline { 2 - 3 } & $\begin{array}{r}\text { wavelength } \\
(\mathrm{nm})\end{array}$ & $\begin{array}{r}\text { energy } \\
(\mathrm{eV})\end{array}$ & & \\
\hline $\mathrm{FX}_{L}$ & 367.12 & 3.3772 & & $\mathrm{Ga}$ \\
$\mathrm{FX}_{T}$ & 367.26 & 3.3759 & & \\
$\mathrm{I}_{0}$ & 367.63 & 3.3725 & & \\
$\mathrm{I}_{1}$ & 367.71 & 3.3718 & & $\mathrm{Surface}$ exciton \\
$\mathrm{I}_{1 a}$ & 368.13 & 3.3679 & & $\mathrm{Zn}$ \\
$\mathrm{I}_{2}^{*}$ & 368.19 & 3.3674 & & $\mathrm{H}$ \\
$\mathrm{SX}^{*}$ & 368.23 & 3.367 & & $\mathrm{Al}$ \\
$\mathrm{I}_{3}^{*}$ & 368.29 & 3.3665 & & $\mathrm{Al}$ \\
$\mathrm{I}_{3 a}$ & 368.34 & 3.3660 & & $\mathrm{Ga}$ \\
$\mathrm{I}_{4}$ & 368.34 & 3.3628 & 46.1 & $\mathrm{In}$ \\
$\mathrm{I}_{5}$ & 368.86 & 3.3614 & & \\
$\mathrm{I}_{6}$ & 368.92 & 3.3608 & 51.55 & \\
$\mathrm{I}_{6 a}$ & 368.96 & 3.3604 & 53 & \\
$\mathrm{I}_{7}$ & 369.01 & 3.3600 & & \\
$\mathrm{I}_{8}$ & 369.03 & 3.3598 & 54.6 & \\
$\mathrm{I}_{8 a}$ & 369.08 & 3.3593 & & \\
$\mathrm{I}_{9}$ & 369.37 & 3.3567 & 63.2 & \\
$\mathrm{I}_{10}$ & 369.76 & 3.3531 & 72.6 & \\
$\mathrm{I}_{11}$ & 370.28 & 3.3484 & & \\
\hline & & & & \\
\hline
\end{tabular}


and the corresponding donor bound exciton line is the energy difference between the $1 s$ ground state and the excited donor state [73; 358], for example $2 s$.

Figure 6.2 shows broad and weak transitions at about $3.29 \mathrm{eV}$ and $3.22 \mathrm{eV}$, which are labeled with DX-LO and DX-2LO, respectively. Those transitions are assigned to phonon replicas, because they are shifted by about $72 \mathrm{meV}$ or an even multiple of this value to lower energies. The difference of $72 \mathrm{meV}$ fits well to the energy of the longitudinal optical phonon (LO) in $\mathrm{ZnO}$ [1]. A more detailed look onto the luminescence of as-grown $\mathrm{ZnO}$ nanowires is given in reference [237].

\subsection{The shallow indium donor}

The contribution of In to the near band gap luminescence of $\mathrm{ZnO}$ was shown by radioactive indium doping in $\mathrm{ZnO}$ single crystals. Figure 6.3 shows the photoluminescence of ${ }^{111} \mathrm{In}$ implanted $\mathrm{ZnO}$ single crystals $^{1}$ [75]. The isotope ${ }^{111} \mathrm{In}$ decays via electron capture into stable ${ }^{111} \mathrm{Cd}$ with a lifetimes of 97,11 h [361]; thus, an element transition from a donor to an isoelectronic element within the $\mathrm{ZnO}$ crystal occurs upon time. Figure 6.3 a shows the decrease of intensity of the $\mathrm{I}_{9}$ line with increasing time, while the intensity of the $\mathrm{I}_{5,6}$ reference lines stays constant. The inset in figure 6.3a shows a plot of the area ratio of the $\mathrm{I}_{9} / \mathrm{I}_{5,6}$ PL lines as a function of time. A single exponential decay (solid line) fits very well the data and yields into a lifetime of 102(15) h matching the lifetime of the implanted ${ }^{111} \mathrm{In}$. Therefore, this PL line cannot be assigned to any implantation related intrinsic defects such as Zn, and $\mathrm{O}$ interstitials or $\mathrm{Zn}$ and $\mathrm{O}$ vacancies, but solely to excitons bound to the donor indium on substitutional $\mathrm{Zn}$ lattice sites. The lattice site of the In atom in $\mathrm{ZnO}$ was determined by perturbed- $\gamma \gamma$-angular-correlation (PAC) [75], which show the incorporation of $\mathrm{In}$ on $\mathrm{Zn}$ lattice site in the majority and a $\mathrm{In}-\mathrm{Zn}_{i}$ defect complex in the minority [75]. Additionally, the sharpness of this line rules out an assignment to the randomly oriented $\mathrm{In}-\mathrm{Zn}_{i}$ complexes [362], and furthermore, acceptor-donor-pairs do not create levels that close at band edges.

Note, the $\mathrm{I}_{9}$ line does not vanish with infinite times, as one would expect, and a constant background intensity $\mathrm{I}_{0}$ has been added to the fit function in Fig. 6.3a. The reason for this is given, on one hand, by the limited mass resolution of the used ion

\footnotetext{
${ }^{1}$ Radioactive ${ }^{111} \mathrm{In}$ was implanted into CrysTec $\mathrm{ZnO}$ single crystals with an ion energy of $400 \mathrm{keV}$ at room temperature. Subsequent annealing of the samples was performed in air at $700{ }^{\circ} \mathrm{C}$ for $45 \mathrm{~min}$ in order to remove the implantation damage. The loss of radioactivity upon this procedure is negligible demonstrating that indium does not significantly diffuse in $\mathrm{ZnO}$ up to $700{ }^{\circ} \mathrm{C}$, in agreement with Refs. [73] and [360].
} 

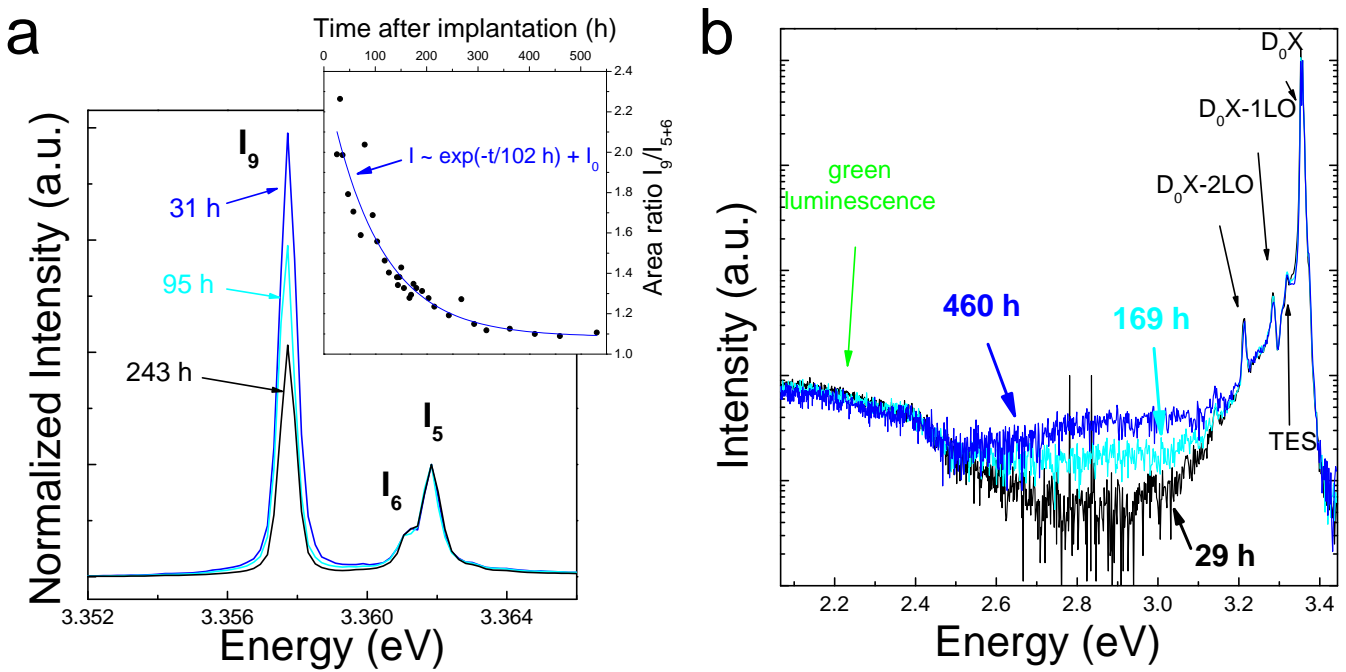

Figure 6.3: Photoluminescence of an ${ }^{111}$ In implanted $\mathrm{ZnO}$ single crystal [75]: (a) near band gap luminescence after $31 h, 95 h$, and $243 h$ reveal an decrease of the $I_{9}$ intensity with the time; inset in (a) shows the time dependent intensity ration of the $I_{9}$ line to the $I_{5,6}$ lines; the survey spectra show an increasing intensity of a new luminescence band between the green band and the near band gap luminescence.

implanter and, on the other hand, by the use of stable ${ }^{115} \mathrm{In}$ for mass calibration of the implanter. Therefore, small amounts of stable ${ }^{115}$ In were coimplanted with the radioactive implantations yielding into the background PL signal. This explanation is supported by the fact that the mass difference of the two In isotopes (115 versus 111) becomes visible in the PL spectra: the $\mathrm{I}_{9}$ line shows a very weak double structure for intermediate times, which is just within the pixel resolution of the used PL setup. Additional implantation experiments with solely stable ${ }^{115} \mathrm{In}$ resulted into the appearance of the $\mathrm{I}_{9}$ line located at $3.3572 \mathrm{eV}$, which is at the most $0.8 \mathrm{meV}$ redshifted compared to ${ }^{111} \mathrm{In}$. This shift is a consequence of the fact that the involved levels in the transition are vibronic levels of the defect in the lattice, and the mass of the donor perturbs the vibronic band [363]. The observed redshift in our experiments is in good agreement with Ref. [363].

While recording the PL spectra over the complete UV and visible range of the radioactive implanted $\mathrm{ZnO}$ sample, an arising broad band centered at $2.85 \mathrm{eV}$ between the band edge and green luminescence was noticed, as shown in figure 6.3b. Again, the increase of this band can be clearly correlated with the lifetime of the implanted isotope. The interpretation of this feature is not unambiguous. One possible explanation is that the radioactive atom receives during the electron capture transition enough recoil energy in order to be reimplanted into the lattice leaving behind 
a vacancy and creating further defects, which causes the new luminescence band. However, the recoil energy can be calculated to 3 and $6 \mathrm{eV}$ and, on the other hand, the displacement energies for $\mathrm{O}$ and $\mathrm{Zn}$ atoms in $\mathrm{ZnO}$ are 45 and $18 \mathrm{eV}$ [364; 365], respectively. Therefore, such a scenario is very unlikely, but cannot be completely ruled out. The second and more reliable reason is that the aborning Cd atoms, which are nominally isoelectronic in $\mathrm{ZnO}$, cause deep levels in the band gap. This effect is not uncommon and has been already observed for $\mathrm{Hg}$ in $\mathrm{ZnO}$ [366]. It can be attributed either to a lattice deformation due to the atomic size difference or to the difference in electronegativity between impurity and host atom [367]. The fact of an arising band rather than a sharp line indicates a nonuniform surrounding of the isoelectronic Cd impurities.

\subsection{Situation after transition metal implantation}

Figure 6.4 presents the luminescence properties of transition metal and argon implanted $\mathrm{ZnO}$ samples with different origins, where the CrysTec single crystals, nanowires, and pulsed laser deposited thin films are abbreviated with CT, NW, and PLD, respectively. Except the Ar implanted PLD sample, all samples implanted with transition metals or argon show an identical trend in their optical properties. It should be noted at this point that the vanadium and argon implanted pulse laser deposited films were annealed under pure oxygen ambient at $800{ }^{\circ} \mathrm{C}$ [49]. All other samples were annealed in air. The luminescence of implanted nanowires in figure 6.4 was recorded after annealing at $700{ }^{\circ} \mathrm{C}$ [50], whereas the luminescence of implanted CrysTec single crystals in figure $6.4 \mathrm{~b}$ was recorded after annealing at $900{ }^{\circ} \mathrm{C}$.

All samples show a dramatic increase of the intensity in the visible spectral region compared to the near band gap luminescence. The comparison of figure $6.1 \mathrm{~b}$ with figure 6.4 indicates a turnaround of the green luminescence band from a Gaussian like shape into a structured shape. However, some samples show an additional deep luminescence band in the red-yellow spectral region, which is entitled in the following as 'red-yellow band'. The argon implanted PLD film shows only this red-yellow luminescence band, and no indication of a green luminescence band (see figure 6.4b). The nature behind both deep luminescence bands are discussed in the next section.

The Fe and Co implanted $\mathrm{ZnO}$ samples show after annealing some additional transitions (see figure 6.4). The spectral positions of those transitions depend on the transition metal ion, whereby this luminescence bands are centered at about $675 \mathrm{~nm}$ and $715 \mathrm{mn}$ for Co implanted samples and for Fe implanted samples, respectively. 

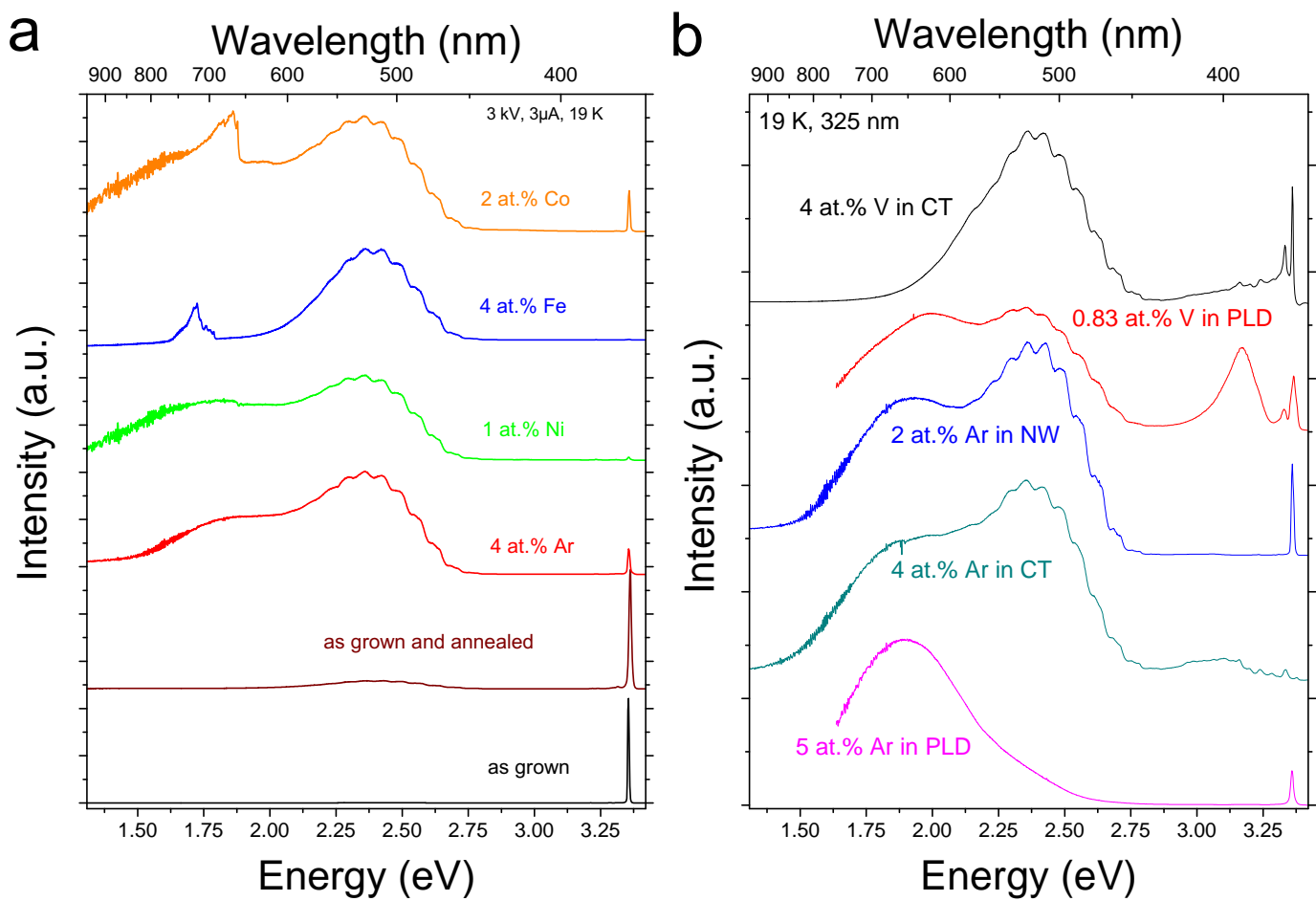

Figure 6.4: Luminescence after TM ion implantation into $\mathrm{ZnO}$ and annealing shows a dramatic increase of the structured green luminescence. A comparison of the cathodoluminescence is shown in (a) between TM implanted ZnO nanowires, an argon implanted reference sample and as-grown $\mathrm{ZnO}$ nanowires. (b) shows an identical trend for TM and Ar implanted ZnO samples with different origin.

These luminescence bands are assigned to intra-shell luminescence of the transition metal ions inside the $\mathrm{ZnO}$ matrix $[21-23 ; 25 ; 368]$ and are discussed in section 6.4.

\subsection{Deep center luminescence}

Nearly a dozen different deep center luminescence bands were observed in $\mathrm{ZnO}$ with maxima between $1.5 \mathrm{eV}$ and $2.7 \mathrm{eV}$ [65; 369]. Up to now the origin of those luminescence bands is discussed controversially, see for instance some $\mathrm{ZnO}$ review articels $[63 ; 98 ; 370]$. Commonly deep center luminescence is assigned to oxygen vacancies $V_{O}[70 ; 71 ; 323 ; 350]$, copper impurities $C u_{Z n}[90 ; 351 ; 371]$, zinc vacancies $V_{Z n}[369 ; 372 ; 373]$, and more complex defect centers $[49 ; 64 ; 374]$. This section discusses the nature of the observed green and red-yellow luminescence bands, which are visible in the figures 6.1 and 6.4 . 


\subsubsection{The oxygen vacancy in $\mathrm{ZnO}$}

The oxygen vacancy generates deep donor states within the band gap of $\mathrm{ZnO}$ [72; $353 ; 375 ; 376]$. The charge state of the oxygen vacancy depends on the position of the Fermi level within the band gap [375; 376], whereby only the neutral $V_{O}^{0}$ or double positive charged $V_{O}^{2+}$ oxygen vacancies are thermodynamical stable in $\mathrm{ZnO}$. The simulations of Erhart et al. and Van de Walle predict a direct transfer from the $2+$ charge state to the neutral charge state if the Fermi level lies more than $\sim 2.7 \mathrm{eV}$ upside the valence band $[375 ; 376]$. However, the single ionized oxygen vacancy $V_{O}^{+}$ is metastable and could be created by excitation with light $[323 ; 350 ; 377]$. The $V_{O}^{2+}$ can trap a photo-activated electron and change to the single charged oxygen vacancy [323]. The single positive charged oxygen vacancy is paramagnetic in contrast to $V_{O}^{0}$ and $V_{O}^{2+}$; and can be detected by electron paramagnetic resonance (EPR) [323; 350]. Vanheusden et al. discovered a correlation between the intensities of the single charged oxygen vacancy EPR signal and the Gaussian shaped green luminescence band of $\mathrm{ZnO}$ [323; 350]. Both show an increase with increasing annealing temperature in forming gas, and vice versa a decrease during annealing in oxygen atmosphere $[323 ; 350]$. The green luminescence of the single positive charged $V_{O}^{+}$is assigned by Vanheusden et al. to a recombination of a photo-excited hole in the valence band transferring the $V_{O}^{+}$center back into the $V_{O}^{2+}$ center [350].

Leiter et al. introduced a intra-defect recombination model for the green oxygen vacancy luminescence $[70 ; 71]$, which is outlined in figure 6.5 a. The ground state of a neutral oxygen vacancy is a singlet state with two electrons and can be excited into an excited singlet state [70; 71], which is indicated by the red arrow in figure 6.5a. Both singlet states are diamagnetic, and could not be observed during ODMR ${ }^{2}$ measurements. However, the excited singlet state relaxes into an excited triplet state (blue arrow in figure 6.5a) [70; 71], which is paramagnetic and could be detected by ODMR. This state relaxes into the ground state by emission of a photon with an energy of $2.45 \mathrm{eV}[70 ; 71]$, which corresponds to the peak position of the green luminescence band. Leiter et al. used the configuration coordinate model to describe the half-width of the green luminescence band, which is than given by $[71 ; 378]$ :

$$
H_{1 / 2}=2.36 S \hbar \omega_{g} \sqrt{\operatorname{coth}\left(\frac{\hbar \omega_{g}}{2 k_{B} T}\right)} .
$$

\footnotetext{
$\overline{2}$ ODMR: Optically Detected Magnetic Resonance
} 
The factor $S$ in equation 6.1 is the Huang-Rhys factor and $\hbar \omega_{g}$ is the mean phonon energy in the ground state.

Figure 6.5 shows a scheme of the configuration coordinate model with the states $k$ and $m$ [378]. In a simple model those two states $k$ and $m$ are the excited and ground state of one defect [378; 379], respectively. Whereby, an electric dipole transition may occur between these states [379]. It is assumed that both states have discrete quantum states $[71 ; 378 ; 380]$, which are indicated as red or orange lines in figure $6.5 \mathrm{~b}$. The energy $E$ in the configuration coordinate diagram in figure 6.5 is plotted as a function of the vibration mode (effective nuclear coordinate) [378; 379]. The calculation of the defect electronic states is done with the group theory and is comparable to $3 d$-shell degeneration of transition metals (see discussion in section 2.2) and depends on the charge distribution of both states shown in figure 6.5b [379]; especially the Jahn-Teller distortion is of high importance. The excitation of the defect from its ground state into the excited state leads to a relaxation of the lattice resulting in a reconfiguration of both states [379]. As the excitation of the state would occur from point $\mathrm{A}$ to $\mathrm{B}$ at the equilibrium position $R_{G}$ (see figure $6.5 \mathrm{~b})$, whereby the defect recombines between point $\mathrm{C}$ and $\mathrm{D}$ at the equilibrium position $R_{E}$ [378; 379]. Therefore, a stokes shift of such color centers could be observed between an absorbed photon and an emitted photon [378]. One result of the configuration coordinate model is the Gaussian shape of the emission and its temperature dependence of the half-width shown in equation 6.1 [378], which resuls from the thermal occupation probability of various vibrational levels. Shionoya et al. had developed an expression to describe temperature dependent shift of peak position of a transition corresponding to the configuration coordinate model [381]:

$$
\Delta E_{e m}=\left(\frac{\omega_{g}^{2}-\omega_{e}^{2}}{\omega_{e}^{2}}+\frac{8 \omega_{e}^{4}}{\omega_{e}^{2}\left(\omega_{g}^{2}+\omega_{e}^{2}\right)}\right) \cdot\left(\frac{E_{a b}-E_{e m}}{E_{e m}}\right) k_{B} T
$$

where $\omega_{e}$ is the phonon frequency in the excited state; $E_{a b}$ and $E_{e m}$ are the energies of the absorption and emission maximum at $0 \mathrm{~K}$, respectively. With increasing temperature the occupation of vibrational levels changes due to thermal excitation, which results in the temperature shift of the peak energy given in equation 6.2 [381].

Leiter et al. show that the configuration coordinate model is valid for the intradefect recombination of the oxygen vacancy luminescence model [71]. The authors show that the peak position energy as well as the half-width of the green luminescence band increases with increasing temperature [71]. However, annealing in air or in oxygen ambient should reduce the concentration of oxygen vacancies due to the 

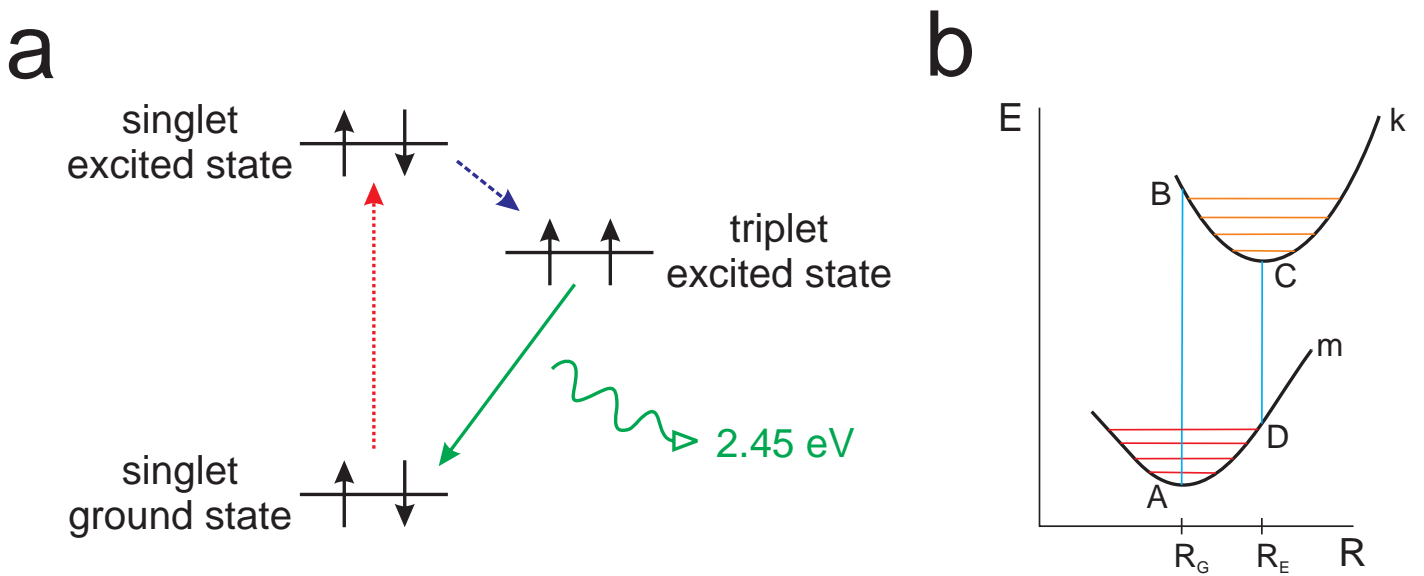

Figure 6.5: (a) Scheme of the intra-defect recombination of the oxygen vacancy represents the emission of the green luminescence of $\mathrm{ZnO}$ by recombination from excited triplet state to the singlet ground state (adapted from [70; 71]). (b) is the schematic configuration coordinate model, which is used to describe the intra-defect luminescence of the oxygen vacancy in (a) (adapted from [378]).

in-diffusion of oxygen. As a result the green luminescence band of the oxygen vacancy decreases, which is visible in figure 6.1 for air annealed virgin CrysTec single crystals.

This explanation is further supported by the results from DLTS measurements. In figure 6.6 DLTS spectra recorded for three different rate windows on the 5at.\% Vimplanted $\mathrm{ZnO}$ sample are shown [49]. Standard Arrhenius evaluation (inset of figure $6.6 \mathrm{~b})$ of the two DLTS features in the low $\left(E_{a 1}\right)$ and high $\left(E_{a 2}\right)$ temperature range reveals a thermal activation energy and apparent capture cross section amounting to $E_{a 1}=236 \mathrm{meV}$ and $\sigma_{i 1}=2.6 \cdot 10^{17} \mathrm{~cm}^{2}$ and $E_{a 2}=672 \mathrm{meV}$ and $\sigma_{i 2}=2.1 \cdot 10^{13} \mathrm{~cm}^{2}$, respectively. If the green luminescence is due to oxygen vacancies, a third electron trap with a thermal activation energy of about $530 \mathrm{meV}$ should appear according to the references [382-384]. However, since this is absent for the V-implanted and subsequently annealed $\mathrm{ZnO}$, one can conclude that the amount of oxygen vacancies is marginal and thus does not contribute to the luminescence in any of the investigated samples. Electron traps with similar thermal activation energies like $E_{a 2}$ were observed in Co- and $\mathrm{Mn}$-doped $\mathrm{ZnO}$, as well as in $\mathrm{ZnO}$ varistors doped with $\mathrm{Co}$ and other impurities. Given the fact that this trap appears in $\mathrm{ZnO}$ with different impurities, it has been assumed that this trap is generated by extrinsic or intrinsic defects or dislocation loops $[385 ; 386]$.

The decrease of the oxygen vacancy concentration due to annealing in air/oxygen ambient does not explain the increasing intensity of the structured green lumines- 

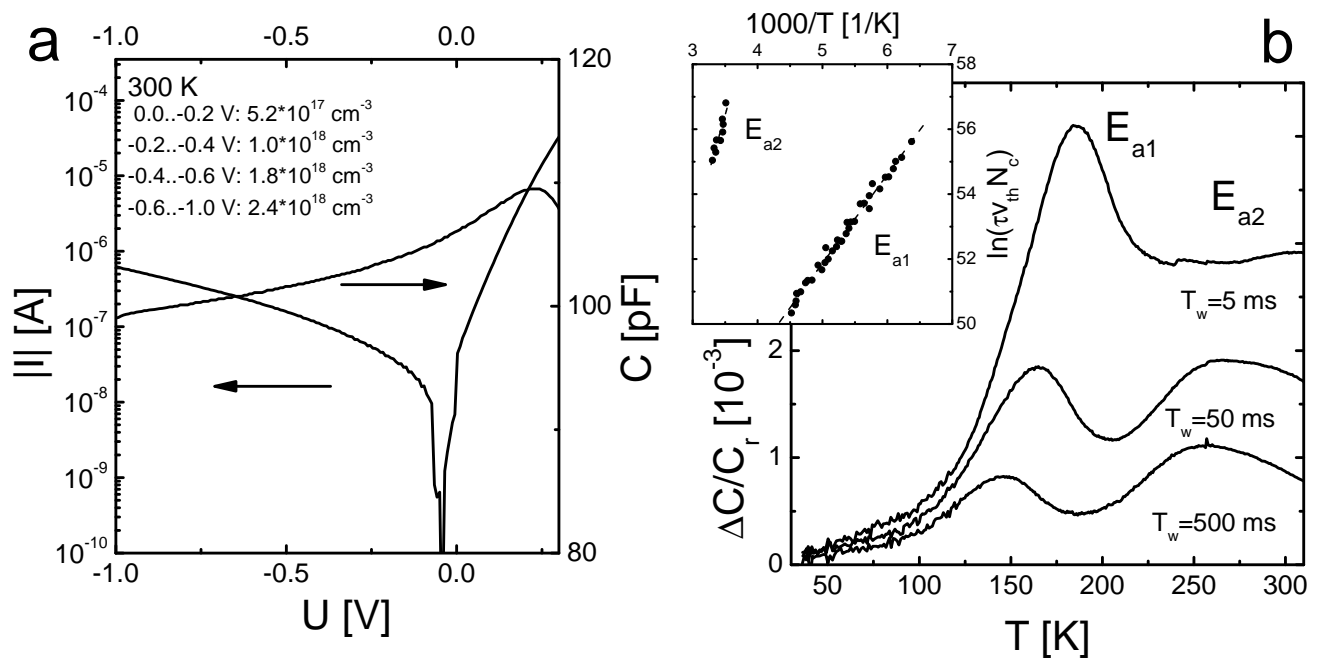

Figure 6.6: DLTS spectra (b) measured on a Schottky diode (a) fabricated from the 5 at.\% vanadium doped $\mathrm{ZnO}$ sample at several rate windows $T_{w}=5,50,500 \mathrm{~ms}$ [49]. The temperature of the two DLTS peaks increases with decreasing rate window. (Inset) Standard Arrhenius evaluation of the low and high temperature DLTS peak reveals a thermal activation energy $E_{a}$ and apparent capture cross section amounting to $E_{a 1}=236 \mathrm{meV}$ and $\sigma_{i 1}=2.6 \cdot 10^{17} \mathrm{~cm}^{2}$ and $E_{a 2}=672 \mathrm{meV}$ and $\sigma_{i 2}=2.1 \cdot 10^{13} \mathrm{~cm}^{2}$, respectively. For the $\sigma_{i}$ analysis possible detrapping entropy changes have been neglected.

cence band, first and foremost not the dramatic increase of the structured green luminescence band for all transition metal implanted $\mathrm{ZnO}$ samples. The implantation process may generate a high amount of different defects like zinc vacancies $\left(\mathrm{V}_{Z n}\right)$, oxygen vacancies $\left(\mathrm{V}_{O}\right)$, zinc interstitials $\left(\mathrm{Zn}_{i}\right)$, oxygen interstitials $\left(\mathrm{O}_{i}\right)$ and oxygen or zinc antisite defects $\left(\mathrm{O}_{Z n}, \mathrm{Zn}_{O}\right)$. Therefore, it is necessary to discuss the annealing effect of all ion implantation defects, which is done consistent with the 'new' red-yellow and structured green luminescence band.

\subsubsection{Red-yellow luminescence band in $\mathrm{ZnO}$}

Figure 6.7a shows the photoluminescence of an Ar implanted PLD ZnO thin film in comparison to $\mathrm{V}$ implanted PLD $\mathrm{ZnO}$ thin films, which were annealed in oxygen ambient at $800^{\circ} \mathrm{C}$ [49]. In contrast to the $\mathrm{V}$ implanted samples the Ar implanted sample shows an intense broad band in the red-yellow with only one pronounced maximum located at $1.9 \mathrm{eV}$ with a full width at half maximum (FWHM) of about $470 \mathrm{meV}$ (see figure 6.7a). Ar implanted $\mathrm{ZnO}$ nanowires show this red-yellow as well as the structured green luminescence band (see figure 6.7b). The intensity ration 

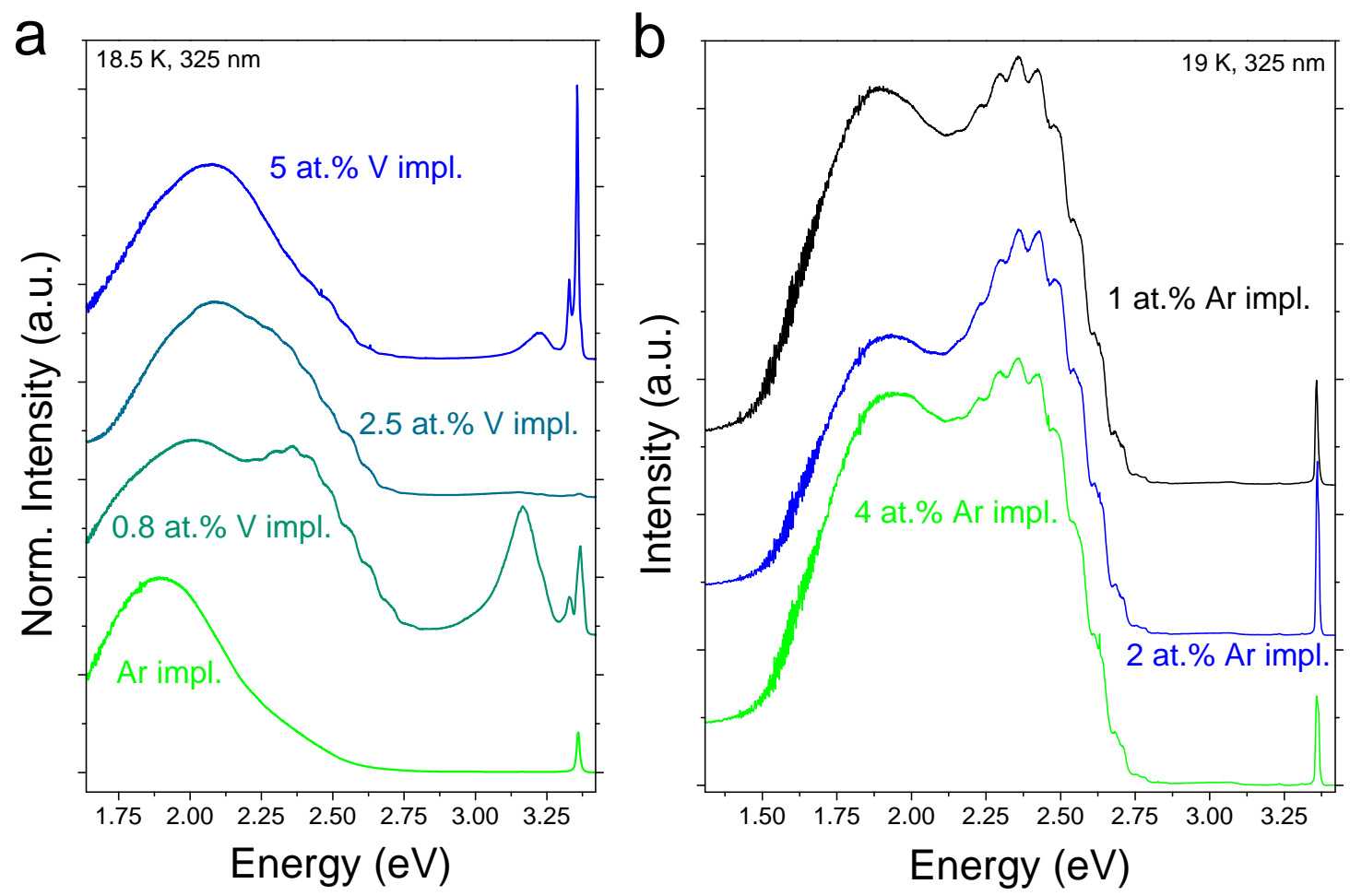

Figure 6.7: (a) shows the photoluminescence of $P L D$ grown $Z n O$ thin films after ion implantation of $V$ or Ar, and subsequent annealing in oxygen ambient [49]. (b) shows the photoluminescence of Ar implanted $\mathrm{ZnO}$ nanowires, which are annealed in air.

between both bands does not really change with increasing implantation dose. However, argon as noble gas diffuses out after the ion implantation, at the latest during the annealing process. Therefore, the red-yellow luminescence could only be assigned to a defect, which was formed during the ion implantation and annealing process. This statement is further supported by the luminescence of the $\mathrm{V}$ implanted PLD samples in figure 6.7, where the red-yellow luminescence is visible, too. Luminescence in the red-yellow spectral range was also found by Adekore et al. after argon irradiation and was generally assigned to 'ion implantation created defects' [387]. A similar luminescence band in the orange spectral range was observed in bulk ZnO, whereby the peak position shifts from $1.95 \mathrm{eV}$ at $10 \mathrm{~K}$ to $2.12 \mathrm{eV}$ at room temperature [369]. Reshchikov et al. assigned this broad luminescence to a transition from a shallow donor to a deep acceptor [369], but had not given an identification of the chemical nature of the involved impurities. The observed shift to higher peak positions would support the configuration coordinate model, if the half-width of this band increases with increasing temperature, which was not shown. Figure 6.8 reveals 


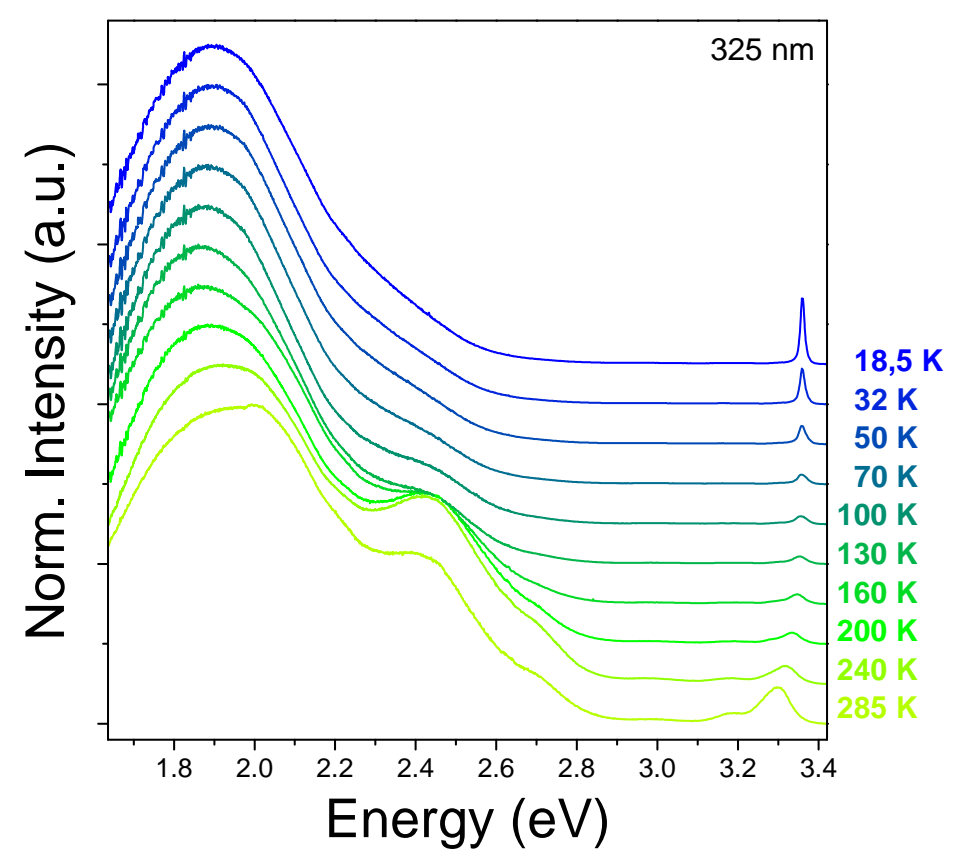

Figure 6.8: The temperature dependent photoluminescence of the Ar implanted $\mathrm{ZnO}$ PLD thin film shows a shift of the red-yellow luminescence band to higher peak positions with increasing temperature [49].

a shift of the peak position to higher energies of the red-yellow luminescence with increasing temperature as well as an increase of the half-width.

During annealing interstitial oxygen can recombine with an oxygen vacancy as well as an interstitial zinc with a zinc vacancy. The annealing procedure in oxygen or air ambient would therefore result in a reduction of oxygen vacancies due to the indiffusion of oxygen from the ambient [323; 350], as well. If both competing processes, recombination and oxidation, take place at the same time, the oxygen vacancy concentration is reduced markedly, whereas interstitial oxygen remains in the crystal. A thermal ionization energy of $1.9 \mathrm{eV}$ was determined for interstitial oxygen ions in tetrahedral surroundings $\left(\mathrm{O}_{i, \text { tetr }}^{2-}\right)$ using electron spin resonance (ESR) and PhotoESR measurements [388]. This energy is similar to the position of the red-yellow luminescence band. It can be assumed that the interstitial oxygen in tetrahedral surroundings $\left(\mathrm{O}_{i, \text { tetr }}\right)$ introduces a deep defect state within the band gap of $\mathrm{ZnO}$ resulting in the luminescence in the red-yellow spectral range. Studenikin et al. and Wenckstern et al. observed this red-yellow luminescence in $\mathrm{ZnO}$ samples grown under oxygen rich conditions during PLD growth [389; 390]. The temperature dependence of the red-yellow luminescence shown in figure 6.8 supports the configuration coor- 

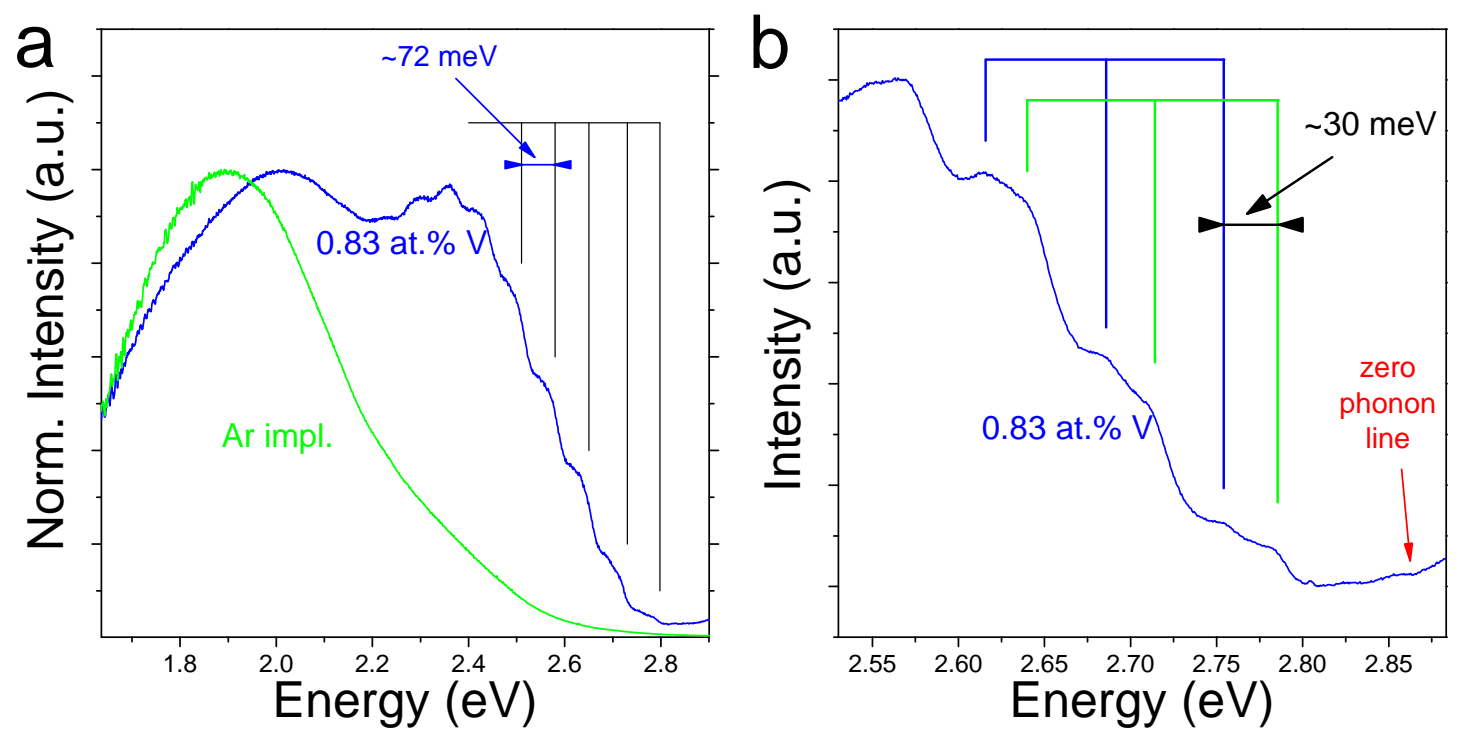

Figure 6.9: (a) The PL spectra of the broad modulated luminescence band recorded on the 0.8 at.\% V-implanted $\mathrm{ZnO}$ sample in comparison to the Arimplanted reference sample. (b) High-resolution PL spectrum of the 0.8 at.\% $\mathrm{V}$ implanted sample showing an extremely weak zero phonon line together with two modulations which are separated by $30 \mathrm{meV}$.

dinate model for this luminescence transition, which is either a transition form a shallow donor to a deep acceptor (as supposed by Reshchikov et al. [369]) or possibly an intra-defect transition such as the oxygen vacancy model from Leiter et al. $[70 ; 71]$.

For higher temperatures a green Gaussian shaped luminescence band emerges in the Ar implanted $\mathrm{ZnO}$ PLD thin film in figure 6.8. The possible nature of this band is discussed later.

\subsubsection{Dingle model}

All TM-implanted $\mathrm{ZnO}$ samples as well as all Ar-implanted $\mathrm{ZnO}$ nanowires and CrysTec samples show a structured green luminescence band at low temperatures after annealing in air/oxygen ambient (see figure 6.4). However, the luminescence of the Ar implanted ZnO PLD thin film shows no green luminescence at low temperature (see figure 6.4), only the red yellow luminescence appears after the irradiation. Figure 6.9a shows a comparison of the deep luminescence bands between the Ar implanted ZnO PLD thin film and the 0.83 at.\% V implanted ZnO PLD thin film. The edges are separated by about $72 \mathrm{meV}$ and are comparable to the energy of the lon- 
gitudinal optical phonon in $\mathrm{ZnO}$. The zero phonon line is located at about $2.86 \mathrm{eV}$, visible as a small hump, and is indicated in figure $6.9 \mathrm{~b}$. On closer examination, the structured green luminescence band shows a fine structure with an separation of about $30 \mathrm{meV}$ from every phonon edge (see figure $6.9 \mathrm{~b}$ ).

This specific modulated luminescence was early assigned to copper impurities in $\mathrm{ZnO}[90 ; 351]$. A copper impurity $\mathrm{Cu}^{2+}$ on zinc site acts as an acceptor in $\mathrm{ZnO}$ with a hole in the $3 d$-shell. Dingle [90] supposed an excited copper state $\left(\mathrm{Cu}^{+}\right)^{+}$where the $3 d$-shell is filled with electrons and the excited hole remains in the environment of the copper impurity and associates it with the four surrounding oxygen atoms. Browser et al. noted, that $\left(\mathrm{Cu}^{+}\right)^{+}$complex can only be formed under optical excitation of the $\mathrm{Cu}^{2+}$ [391]. During recombination, this excited hole jumps back into the copper $3 d-$ shell and as a result of the high shielding of the copper $3 d$-shell a narrow luminescence transition is expected. High-resolution photoluminescence excitation spectra show three zero phonon lines located at about $2.86 \mathrm{eV}$, which are declared with $\alpha, \beta$ and $\gamma$ [391-393]. Thereby, $\alpha$ and $\beta$ are doublet transitions with a separation by about $0.15 \mathrm{meV}[90 ; 363 ; 391-393]$. The intensity ratio of these two lines is in good

agreement with the ratio of the two natural copper isotopes ${ }^{63} \mathrm{Cu} /{ }^{65} \mathrm{Cu}$. The $\gamma$ line shows no isotope splitting and is by a factor of ten broader than the $\alpha$ and $\beta$ line [363]. Photoluminescence spectra show only the $\alpha$ line and a second zero phonon line at about $438.5 \mathrm{~nm}(\sim 2.83 \mathrm{eV})$, which is not understood within the Dingle model $[351 ; 391 ; 392]$.

\subsubsection{Configuration coordinate model for the structured green luminescence band of $\mathrm{ZnO}$}

Dingle's model describes very well the sharp line doublet at $2.86 \mathrm{eV}$ and their phonon replica. But a detailed look on the intensities of this lines and the intensity of the subsequent edge of all reported photoluminescence spectra of the structured green luminescence band shows a great variance [90; 351; 371; 391; 392]. Dingle's publication reveal a zero phonon line doublet, which has an intensity nearly as strong as the subsequent edge [90]. Luminescence spectra of Broser et al. and Garces et al. show a much lower intensity of the zero phonon line doublet $[371 ; 391]$. Figure $6.9 \mathrm{~b}$ shows a high-resolution PL spectrum of the modulated structure of the 0.8 at.\% V doped $\mathrm{ZnO}$ sample revealing an extremely weak zero phonon line and its phonon replicas with much higher intensity. However, a sharp line doublet at $2.86 \mathrm{eV}$ could not be observed in any TM implanted $\mathrm{ZnO}$ sample. In addition, Deicher et al. discussed first results of radioactive copper implanted $\mathrm{ZnO}$ with emphasis on the 
structured green luminescence band [394]. The intensity increase of the structured green band with the decay of the radioactive copper isotop ${ }^{64} \mathrm{Cu}$ to ${ }^{64} \mathrm{Ni}$ or ${ }^{64} \mathrm{Zn}$ revealing a more complex nature of the structured green luminescence band [394].

An alternative model for the modulated green band considers a transition from a shallow donor or the conduction band to a deep level [374]. A comparable transition occurs in the yellow luminescence band of GaN with a similar fine structure $[64 ; 380$; 395]. The yellow luminescence of $\mathrm{GaN}$ is described by a configuration coordinate (CC) model, where a deep center has its own vibrational states, characterized by a local phonon energy $[380 ; 395]$. The advantage of the CC model is the possibility to explain the width of the yellow band in GaN and the green band in $\mathrm{ZnO}$ [64]. The energies of these states are given by [64]:

$$
E_{G}-\left(E_{D C}+\eta E_{l o c}\right)
$$

where $E_{l o c}$ is the local phonon energy, $E_{D C}$ the ground state energy of the deep center, $E_{G}$ the band gap of $\mathrm{ZnO}$ and $\eta$ an integer. The broad emission band is then described by a series of possible donor - deep center transitions [64]:

$$
E_{\eta}=E_{G}-\left[E_{D}-\left(E_{D C}+\eta E_{l o c}\right)\right]
$$

where $E_{D}$ is the donor binding energy. The longitudinal-optical (LO) phonon $\left(E_{L O}\right)$ may also be involved in this transition; thus, in the CC model the transition energy amounts to [64]:

$$
E_{\eta}=E_{G}-\left[E_{D}-\left(E_{D C}+\eta E_{l o c}\right)-\eta^{\prime} E_{L O}\right]
$$

where $\eta^{\prime}$ is an integer [374]. Reynolds et al. supposed that this green fine structure is a superposition of two transitions from two different shallow donors to the same deep center. It is assumed that the donors have binding energies of about $60 \mathrm{meV}$ and $30 \mathrm{meV}$ resulting into a separation of the fine structure of about $30 \mathrm{meV}$. This fact describes well the small hump at $\sim 2.83 \mathrm{eV}$ in the luminescence of the V-doped $\mathrm{ZnO}$ and the shift from the zero phonon line as indicated in figure $6.9 \mathrm{~b}$.

Temperature dependent PL data of the 0.8 at.\% and 5 at.\% V-implanted ZnO PLD samples are summarized in figure 6.10(a) and (b), respectively. The intensity of the band-edge luminescence decreases with increasing temperature and vanishes at room temperature (RT); whereas, the Ar-implanted ZnO (figure 6.8) shows still ultraviolet emission at RT. Simultaneously, one can observe a decrease in the same manner of the structured green luminescence band in all $\mathrm{V}$-implanted $\mathrm{ZnO}$, whereby 


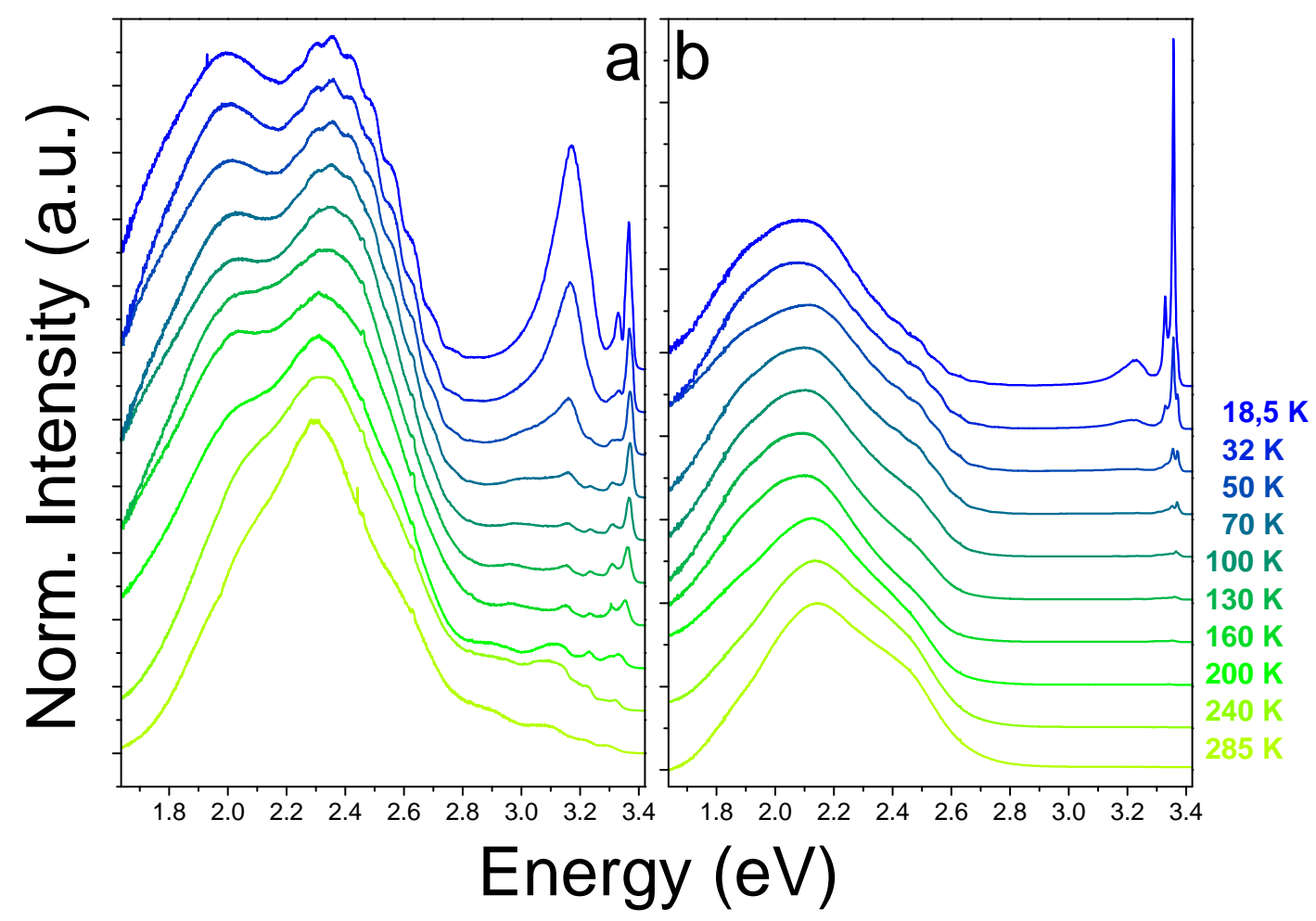

Figure 6.10: The temperature dependent photoluminescence measurement of (a) the 0.8 at.\% and (b) 5 at.\% vanadium doped $\mathrm{ZnO}$ reveals a decreasing intensity of the ultraviolet luminescence with increasing temperature. c) The intensity of green luminescence recorded on the Ar-implanted $\mathrm{ZnO}$ reference sample increases with increasing temperature.

no structure could be observed on the green luminescence band at temperatures above $100 \mathrm{~K}$. The change of the green luminescence band shape with increasing temperature supports assumption of a shallow donor-deep acceptor transition of Reynolds et al [374]. The shallow donors are ionized at elevated temperature. The intensity of the sharp transitions in the near band gap luminescence decreases with increasing temperature, and vanishes at about $100 \mathrm{~K}$ (see section 4.4 in reference [237]). Therefore, it is to assume that the green luminescence band is composed of the donor-acceptor transition and a band to acceptor transition. The green luminescence is dominated by the donor-acceptor transition at low temperatures, which results in the structured shape of the green luminescence at low temperatures. Due to the ionization of the shallow donors, the fraction of the band to acceptor transition in the green luminescence band increases and would be the main luminescence channel above $100 \mathrm{~K}$. However, the decrease in luminescence intensity is spectrally resolved 


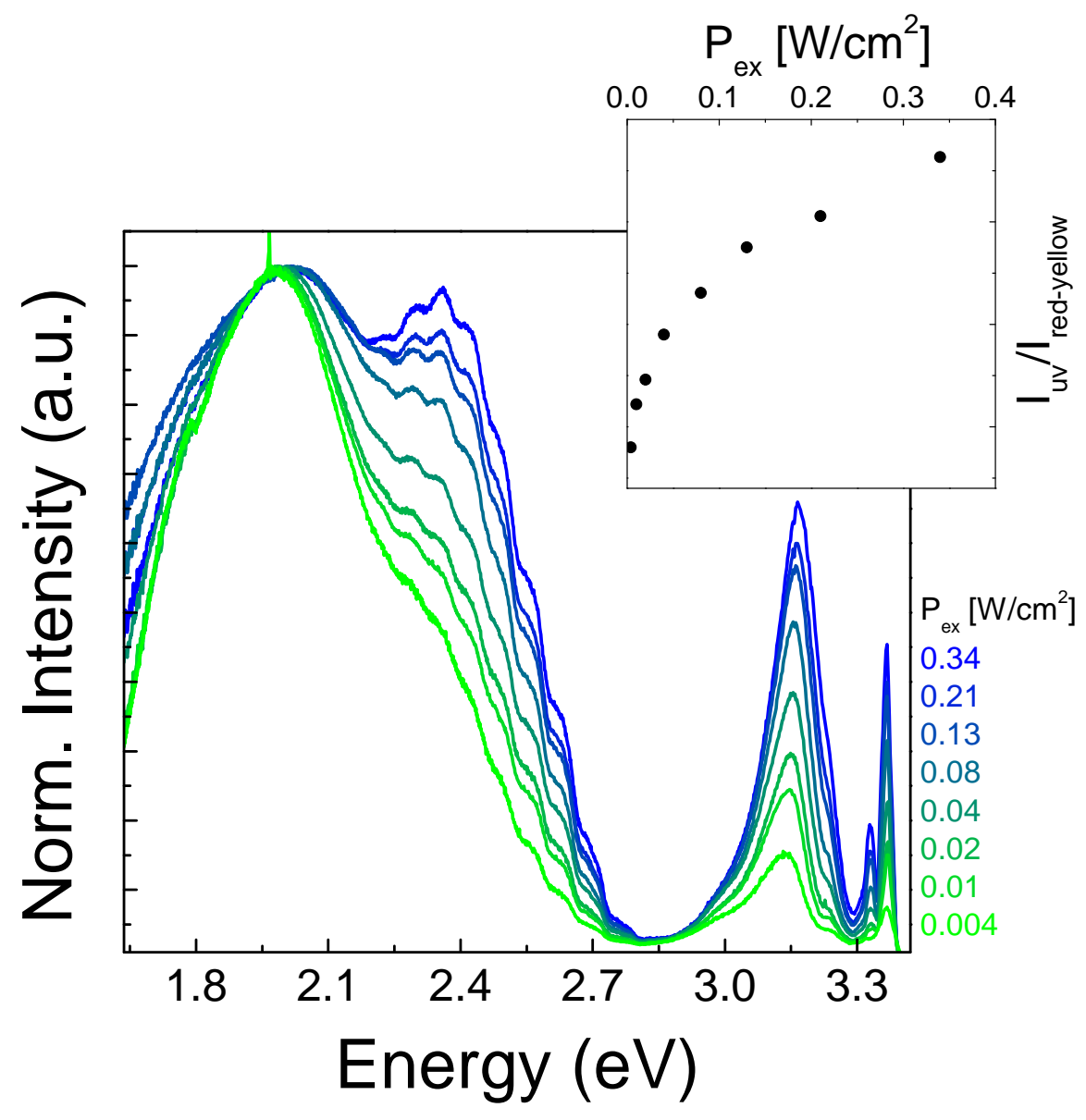

Figure 6.11: Power dependent PL measurements recorded at $18.5 \mathrm{~K}$ on the 0.8 at.\% V-implanted $\mathrm{ZnO}$ sample. The inset shows the ratio between the $U V$ and red-yellow luminescence in dependence on excitation intensity.

and is stronger in the red-yellow than in the green spectral range. The thermal quenching of a luminescence transition with increasing temperature depends on the imperfection of the specific defect surroundings [396].

Figure 6.11 shows the effect on the excitation intensity on the PL-spectra representative for the 0.8 at.\% $\mathrm{V}$-implanted $\mathrm{ZnO}$ sample. With increasing excitation intensity the intensity of the ultraviolet luminescence increases in comparison to the red-yellow luminescence band (see inset of figure 6.11). A small blue shift is observed for the low energy side due to the superposition of both bands.

Furthermore, due to Coulomb effects the DAP related transition at $3.16 \mathrm{eV}$ is blue shifted [359]. The same excitation dependency was observed for the transitions in the 2.5 at.\% and 5.0 at.\% V-implanted $\mathrm{ZnO}$ (not shown). 


\subsubsection{Interstitial zinc and oxygen in the green luminescence band}

In contrast to the implanted Ar, the implanted transition metals remain in the $\mathrm{ZnO}$ crystal and are isoelectronically incorporated on zinc lattice sites, which is confirmed by emission channeling experiments [83; 84]. Doping of wide band gap materials with transition metal elements can induce intra-shell luminescence of the corresponding transition metal element, but for $\mathrm{ZnO}$ these intra-3d transitions appear at lower energies than the structured green luminescence band [24; 25; 397; 398]. However, the isoelectronic transition metals may generate intrinsic deep levels in the band gap acting as radiative recombination centers. This effect is not uncommon and has been already observed for $\mathrm{Hg}$ and $\mathrm{Cd}$ in $\mathrm{ZnO}$ (see references [75; 366] and section 6.1). It can be attributed either to a lattice deformation due to the atomic size difference or to the difference in electronegativity between impurity and host atom [367]. In that case, one may expect different positions of such defect luminescence bands for the different transition metal elements. This speculation is not consistent with the observation in figure 6.4, the position of the structured green luminescence band is not effected by the chemical nature of the transition metal. Therefore, it is unlikely that the transition metals participate in the structured green luminescence. None the less, the ion implantation of transition metals could play an important role for the generation of one or both defects behind the structured green luminescence band.

The ion implantation of transition metals generates a high concentration of additional zinc interstitials in comparison to argon implantation. Given that zinc interstitials are shallow donors $[353 ; 354]$ and Sann et al. assigned the $\mathrm{I}_{3,3 a}$ to interstitial zinc [352], the luminescence of the donor bound excitons should increase and dominate the luminescence of all transition metal implanted $\mathrm{ZnO}$ samples. Whereby, the green luminescence should not be affected. However, luminescence spectra after ion implantation of transition metals show an oppositional effect, the structured green luminescence increases drastically and dominates the luminescence. The near band gap luminescence plays only a minor role. Mathematical simulations of the total density of states of $\mathrm{Zn}$ rich $\mathrm{ZnO}$ insinuate slight differences depending on the interstitial lattice site [354], due to differences in configuration and interactions with neighboring atoms. Kohan et al. had shown by calculation of the formation energy that zinc has a higher tendency to occupy an octahedral interstitial lattice site than the tetrahedral site [373]. These results suggest, that the concentrations of both zinc interstitial lattice sites differ from each other. Due to experimental observation of 
the concentration dependence of two shallow donors by Look et al. [399], Sun et al. assumed, that interstitial zinc introduces shallow donor level with 31 and $61 \mathrm{meV}$ below the conduction band minimum originated from the tetrahedral and octahedral zinc interstitial [354], respectively. This discrepancy of about $30 \mathrm{meV}$ fits very well the separation, which was shown in figure 6.9. Thus, one can assume that octahedral as well as tetrahedral interstitial zinc are the shallow donors in the configuration coordinate model of Reynolds et al. [374].

During ion implantation also oxygen vacancies, zinc vacancies, antisites, and oxygen interstitial are generated. However, the oxygen vacancy, interstitial oxygen on tetrahedral sites as well as zinc on oxygen site introduce donor states into the band gap $[72 ; 353 ; 354]$. On the other hand, the zinc vacancy, oxygen on zinc site, as well as interstitial oxygen on octahedral sites are deep acceptors $[195 ; 353 ; 354$; $373 ; 400]$. Due to the high concentration of interstitial zinc, the concentration of zinc vacancies should be very low. Therefore, the zinc vacancies should be only a minor factor for the green luminescence band [373]. The in-diffusion of oxygen during the annealing in air/oxygen ambient would not only increase the concentration of tetrahedral oxygen interstitials, the concentration of octahedral oxygen interstitials should increase as well. Kohan et al. supposed that octahedral interstitial oxygen is more stable than the tetrahedral interstitial oxygen due to simulation results of the defect formation energies [373]. This statement implicates a higher concentration of octahedral oxygen interstitial than tetrahedral oxygen interstitials. Octahedral oxygen interstitials are supposed to be deep acceptors introducing levels $0.57 \mathrm{eV}$ and $1.18 \mathrm{eV}$ above the valence band maximum [353; 354], which would fit to the observed data. Furthermore, Zhao et al. determined the acceptor level of antisite oxygen by density functional theory calculations [400], and depending on the charge state of the oxygen antisite energy levels between $0.283 \mathrm{eV}$ and $0.799 \mathrm{eV}$ were calculated.

At this point it can be assumed that the structured green luminescence band is a superposition of two transitions from two different shallow donors to the same deep acceptor, whereby the two different shallow donors are interstitial zinc on octahedral as well as on tetrahedral sites $[49 ; 50]$. The deep acceptor is either the octahedral oxygen interstitial or oxygen on antisites [49; 50]. In section 3.3.1 it was discussed that the $\mathrm{ZnO}$ nanowires grow under zinc rich conditions resulting in the incorporation of interstitial zinc in the lattice, which is confirmed by the near band gap luminescence in figure $6.2 \mathrm{a}$ by the $\mathrm{I}_{3,3 a}$ line [352]. However, the structured green luminescence band in as-grown $\mathrm{ZnO}$ nanowires in figure 6.1a implies that also interstitial oxygen or oxygen on antisite are present, if the assumed model is correct. It is possible that during growth both species are not perfectly installed in the crystal 
lattice, resulting in the formation of interstitial and vacancy defects. Nevertheless, the high growth temperature of about $1000{ }^{\circ} \mathrm{C}$ should anneal those defects during growth by recombination of interstitials and vacancies.

The ion implantation experiment of $\mathrm{Ar}$ into $\mathrm{ZnO}$ supports the in-diffusion of oxygen during annealing in air or oxygen ambient. It can be expected that the oxygen vacancies have an attractive potential for interstitial oxygen as well as oxygen from the ambient during the annealing. The increasing concentration of oxygen interstitials gives rise to the red-yellow luminescence in the PLD grown $\mathrm{ZnO}$ thin film. Whenever interstitial zinc is present in the sample, the structured green luminescence band increases in intensity as well. The last statement explains the increase of the structured green luminescence band for Ar implanted $\mathrm{ZnO}$ nanowires and CrysTec single crystals, which show both the $\mathrm{I}_{3,3 a}$ line of interstitial zinc in figure 6.2 [352]. The concentration of interstitial zinc should not be affected by the Ar implantation, because the ion implantation generated zinc vacancies and interstitials should recombine during annealing. However, the implantation of transition metals increases the concentration of interstitial zinc. Therefore, the intensity of the structured green luminescence band is a function of the concentrations of octahedral oxygen interstitial and interstitial zinc.

\subsection{Intra-shell luminescence of transition metals}

Co and Fe implanted $\mathrm{ZnO}$ samples show additional luminescence bands after annealing (see figure 6.4). It can be seen that the position of those bands depend on the implanted transition metal. For Co this additional band is located at about $1.8 \mathrm{eV}$ and for Fe at about $1.75 \mathrm{eV}$. The shape as well as the position of both bands do not fit to any other defect luminescence band of $\mathrm{ZnO}$, which were discussed so far. However, those two bands fit very well to the intra-shell absorption and luminescence bands of $\mathrm{Co}$ or Fe ions in $\mathrm{ZnO}[22 ; 25]$.

\subsection{1 $\mathrm{Co}^{2+}$ ion}

Cobalt on zinc lattices site are double positive charged ions, therefore the $3 d$-shell has a $d^{7}$ configuration (see table 2.1 in chapter 2 ). Hence, the ground state term of the free $\mathrm{Co}^{2+}$ ion is ${ }^{4} F$ and the first two excited free ion states are ${ }^{4} P$ and ${ }^{2} G$. Figure 6.12 shows the $3 d$-shell configuration of the free $\mathrm{Co}^{2+}$ ion and the degeneration by a tetrahedral crystal field $\left(T_{d}\right.$-symmetry) of a cubic crystal $[21 ; 22 ; 368 ; 401]$. For simplicity, further degenerations by the trigonal $\left(C_{3 \nu}\right)$ crystal field and the spin-orbit 


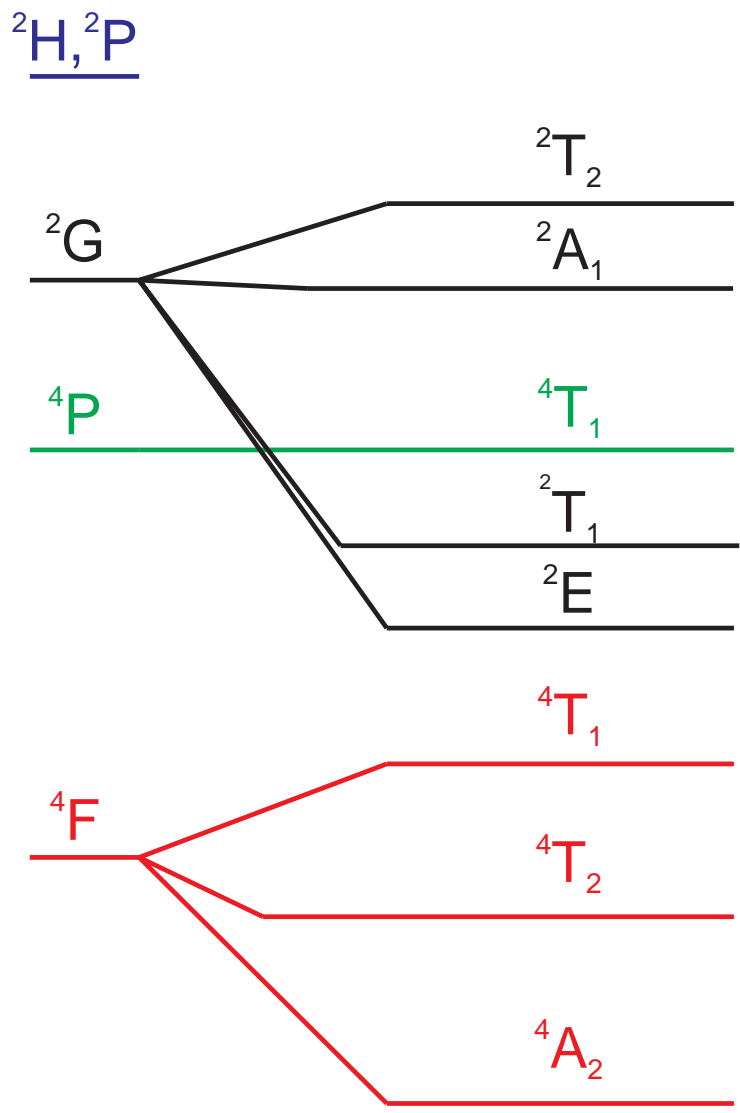

\section{free ion}

Figure 6.12: Configuration of the $\mathrm{Co}^{2+}$ ion in $\mathrm{T}_{d}$ symmetry (adapted from [21; 22; 368; 401]).

interaction are not shown in figure 6.12 , but those perturbations will be discussed and specified in table 6.2. The free ion ground state ${ }^{4} F$ is threefold degenerated by the tetragonal crystal field into the ${ }^{4} A_{2}(F),{ }^{4} T_{2}(F)$ and the ${ }^{4} T_{1}(F)$ term ${ }^{3}[22 ; 368]$. Whereby, the ${ }^{4} T_{2}(F)$ and the ${ }^{4} T_{1}(F)$ term are arranged above the ${ }^{4} A_{2}(F)$ term. It was shown by absorption measurements that all three terms are further degenerated by the trigonal field and spin-orbit interaction $[21 ; 22 ; 368]$. The ground state ${ }^{4} A_{2}(F)$ is twofold degenerated by the trigonal field and forms two Kramers doublets with a splitting between the doublets of about $0.7 \mathrm{meV}[21 ; 368]$. Optical transitions between the ${ }^{4} A_{2}(F)$ ground state and the two excited states were observed in the infra-red in the range of $0.8-1 \mathrm{eV}$ and $0.45-0.55 \mathrm{eV}$ for the ${ }^{4} T_{1}(F)$ and ${ }^{4} T_{2}(F)[21 ; 22$;

\footnotetext{
${ }^{3}$ Commonly, the term levels are labeled with ${ }^{2 S+1} \Gamma_{j}(L)$, where $L$ is the orbital symbol for the free ion state, $2 S+1$ is the spin multiplicity of this level and $\Gamma_{j}$ the irreducible representation of the orbital part for the crystal field degenerated state (for more detail see $[21-23 ; 25 ; 368]$ and references therein).
} 
368], respectively. Due to the fact that those transitions are outside of the working range of our photoluminescence setup, those transitions are not discussed further.

In the visible spectral range a variety of sharp transitions were observed by absorption measurements $[22 ; 88 ; 368]$, which follow from the ${ }^{4} P$ and ${ }^{2} G$ free ion states of the $\mathrm{Co}^{2+}$ ion. The ${ }^{4} P$ free ion state is not split in the $T_{d}$ symmetry, but transforms into ${ }^{4} T_{1}(P)$. Due to the anisotropy of the $\mathrm{ZnO}$ crystal, this term is split by the trigonal field and spin-orbit interaction into at least six states [22; 368]. The ${ }^{2} G$ free ion state is upside the ${ }^{4} P$ state, but the tetragonal crystal field perturbation leads to a fourfold split of this state $[22 ; 88 ; 368]$. However, the ${ }^{2} E(G)$ and ${ }^{2} T_{1}(G)$ terms have lower transition energies than the ${ }^{4} T_{1}(P)$ term, whereby the ${ }^{2} A_{1}(G)$ and ${ }^{2} T_{2}(G)$ terms are positioned above the ${ }^{4} T_{1}(P)[368]$. The ${ }^{2} A_{1}(G)$ term is not further split by the trigonal field component and spin-orbit interaction, which is contrary to the three other ${ }^{2} G$ terms. The ${ }^{2} T_{2}(G)$ term and the ${ }^{2} T_{1}(G)$ term are threefold degenerated by the trigonal field as well as the spin-orbit interaction [368]. The ${ }^{2} E(G)$ term is twofold degenerated and all transtions of the ${ }^{2} E(G)$ states could be observed at about $1.87 \mathrm{eV}$. It was shown by Koidl et al. that all terms of the ${ }^{4} P$ and ${ }^{2} G$ free ion states should be observable between $530 \mathrm{~nm}$ and $660 \mathrm{~nm}$ [368]. Table 6.2

Table 6.2: Collection of energy levels of $\mathrm{Co}^{2+}$ ( $d^{7}$ configuration) 3d-shell in $\mathrm{ZnO}$, which could contribute to the visible intra-shell luminescence including the ${ }^{4} A_{2}(F)$ ground state doublet [22; 368].

\begin{tabular}{lrrrr}
\hline \multirow{2}{*}{ Term } & \multicolumn{2}{c}{ Fine structure } & Spectral position \\
\cline { 2 - 3 } & State & \multicolumn{2}{c}{ Splitting } & \\
\cline { 3 - 4 } & & $\left(\mathrm{cm}^{-1}\right)$ & $(\mathrm{meV})$ & $(\mathrm{eV})$ \\
\hline${ }^{4} A_{2}(F)$ & $E_{1 / 2}$ & 0 & 0 & \\
& $E_{3 / 2}$ & 5.4 & 0.7 & \\
${ }^{2} E(G)$ & $E_{1 / 2}$ & 0 & 0 & 1.8781 \\
& $E_{3 / 2}$ & 38 & 4.7 & 1.8828 \\
${ }^{2} T_{1}(G)$ & & & & $\sim 1.93-1.98$ \\
${ }^{4} T_{1}(P)$ & & & & $\sim 2.01-2.12$ \\
${ }^{4} A_{1}(G)$ & $E_{1 / 2}$ & & & 2.1867 \\
${ }^{2} T_{2}(G)$ & $E_{1 / 2}$ & 0 & 0 & 2.2392 \\
& $E_{3 / 2}$ & 270 & 33.5 & 2.2726 \\
& $E_{1 / 2}$ & 370 & 45.9 & 2.285 \\
\hline
\end{tabular}



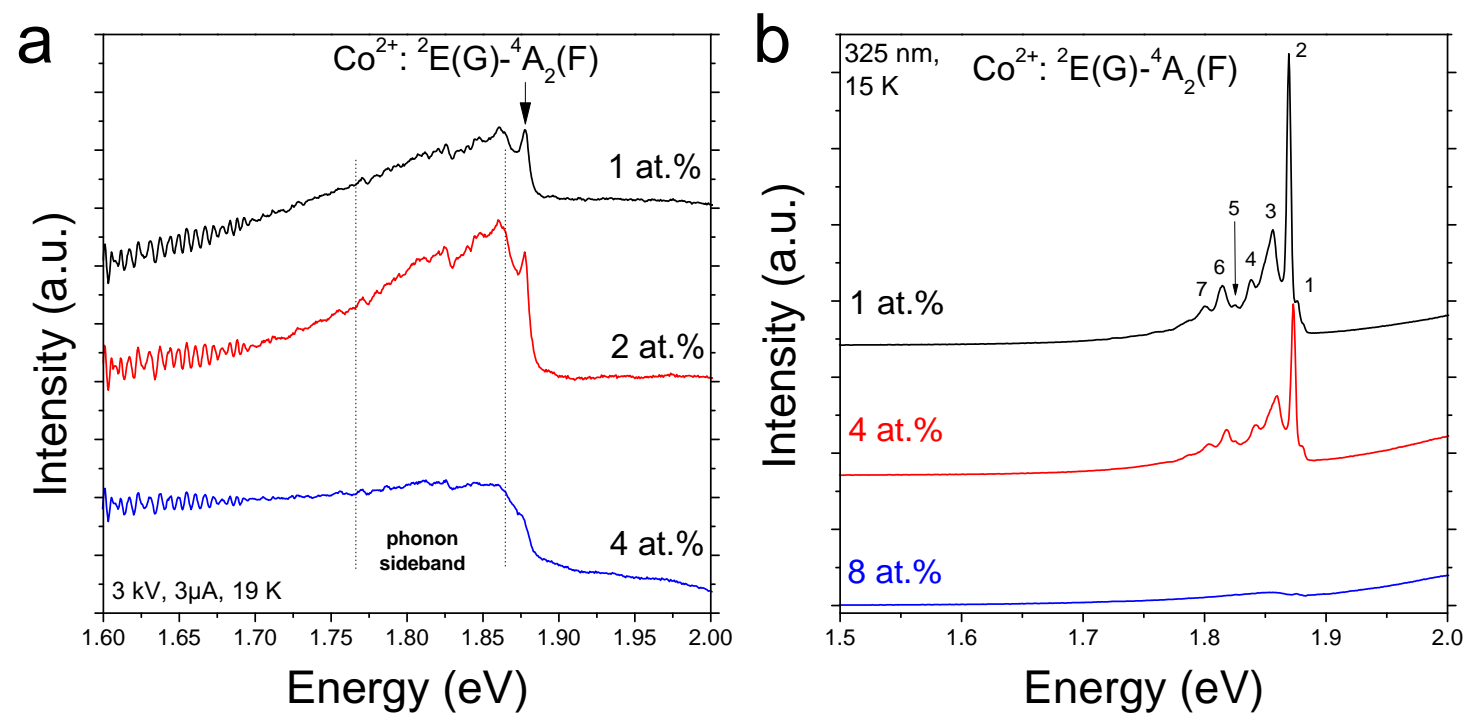

Figure 6.13: The intra-shell luminescence of $\mathrm{Co}^{2+}$ ion within $\mathrm{ZnO}$ nanowires in (a) and $\mathrm{ZnO}$ single crystals in (b) for different Co concentrations [50].

specifies the transition energies of all ${ }^{4} P$ and ${ }^{2} G$ terms to the ${ }^{4} A_{2}(F)$ ground state including the fine structure $[22 ; 368]$.

Figure 6.13 shows the additional luminescence band of Co implanted $\mathrm{ZnO}$ nanowires in (a) and single crystals in (b) as a function of the Co concentration. For low Co concentrations the band is composed of several sharp lines with the zero phonon line at $1.87 \mathrm{eV}$. The zero phonon line can be assigned to a transition from the ${ }^{2} E(G)$ doublet to the ${ }^{4} A_{2}(F)$ ground state doublet [22;368]; and is indicated in figure $6.13 \mathrm{a}$ by the arrow as well as in figure $6.13 \mathrm{~b}$ by the $\# 1$. However, the zero phonon line is only a small shoulder of the line \#2 in all CrysTec ZnO single crystals (see figure 6.13b). The intensity of the transition ${ }^{2} E(G)$ to ${ }^{4} A_{2}(F)$ decreases with increasing Co concentration compared to the consecutive band between 1.76 and $1.86 \mathrm{eV}$ in the Co implanted $\mathrm{ZnO}$ nanowires (see figure 6.13a), and can only be identified as small shoulder in the 4 at.\% Co implanted $\mathrm{ZnO}$ nanowire sample. The Co implanted single crystals show a similar behaviour with increasing Co concentration in figure $6.13 \mathrm{~b}$ (the 8 at.\% Co implanted $\mathrm{ZnO}$ single crystal is shown in more detail in figure 6.14). The low energy side band shows no defined transition in all Co implanted $\mathrm{ZnO}$ nanowires (see figure 6.13), which is totally different to the low energy side band of the Co implanted $\mathrm{ZnO}$ single crystals. In figure $6.13 \mathrm{~b}$ those lines are numbered from \#1 to \#7, their spectral positions are quoted in table 6.3 including the energy difference $\Delta E$ from the zero phonon line $\# 1$. The energy difference 
between line \#1 and \#2 is about $7.3 \mathrm{meV}$, which is greater than the fine structure of the ground state ${ }^{4} A_{2}(F)$ as well as of the ${ }^{2} E(G)$ excited state (see table 6.2). Therefore, the broad band between 1.76 and $1.86 \mathrm{eV}$ could not be assigned to a direct intra-shell transition of the $\mathrm{Co}^{2+}$ ion, but absorption measurements with Co doped $\mathrm{ZnO}$ crystals present a phonon side band on the high energy side $[21 ; 22 ; 88 ; 368]$. Due to the physical differences between absorption and luminescence measurements, the arrangement within the optical spectra changes and the phonon side band in luminescence spectra occurs on the low energy side of the direct transition. Table 6.3 gives a possible identification of the participating phonon in the lines \#2 to \#7, the phonon modes are assigned with respect to the references [88; 303]. However, the assignment of the phonons in table 6.3 should be treated with attention, as these values represent the lattice vibration modes of 'pure' ZnO. The alloying with transition metals should have an effect on the lattice vibration modes. With increasing transition metal content the characteristic of the lattice vibration modes should be changed from 'pure' ZnO towards the characteristic of the corresponding transition metal oxide. Such a mutation of lattice vibration modes was observed by Samanta et al. [135], who observed a shift of the $E_{2}^{\text {low }}$ phonon mode to lower frequencies with increasing Co concentration in $\mathrm{Zn}_{1-x} \mathrm{Co}_{x} \mathrm{O}$ by Raman measurements. In addition, local vibration modes could be a source for some transitions in table 6.3 just as well.

The relative increase of the phonon side band compared to the direct transition with increasing Co concentration is a sign for a lower crystal lattice quality in the

Table 6.3: Phonon side band of the $\mathrm{Co}^{2+}$ intra-shell luminescence, the transition from the ${ }^{2} E(G)$ doublet to the ${ }^{4} A_{2}(F)$ ground state doublet is denoted with $Z P L$ (zero phonon line). $\Delta E$ is the energy difference to the zero phonon line. The phonon modes are identified with respect to the references [88; 303]

\begin{tabular}{lrrr}
\hline Peak \# & $\begin{array}{r}\text { Energy } \\
(\mathrm{eV})\end{array}$ & $\begin{array}{r}\Delta E \\
(\mathrm{meV})\end{array}$ & $\begin{array}{r}\text { Identification } \\
\text { ZPL }\end{array}$ \\
\hline 1 & 1.8766 & & $T A[A], T O[A]$ \\
3 & 1.8693 & 7.3 & $L A[A], L O[A]$ \\
4 & 1.8562 & 20.4 & $B_{1}^{\text {high }}-B_{1}^{\text {low }[\Gamma]}$ \\
5 & 1.8384 & 38.3 & $E_{2}^{\text {high }}[\Gamma]$ \\
6 & 1.8243 & 52.3 & $L O[\Gamma]$, \\
7 & 1.8145 & 62.1 & $T A+T O[H, M]$ \\
\hline
\end{tabular}




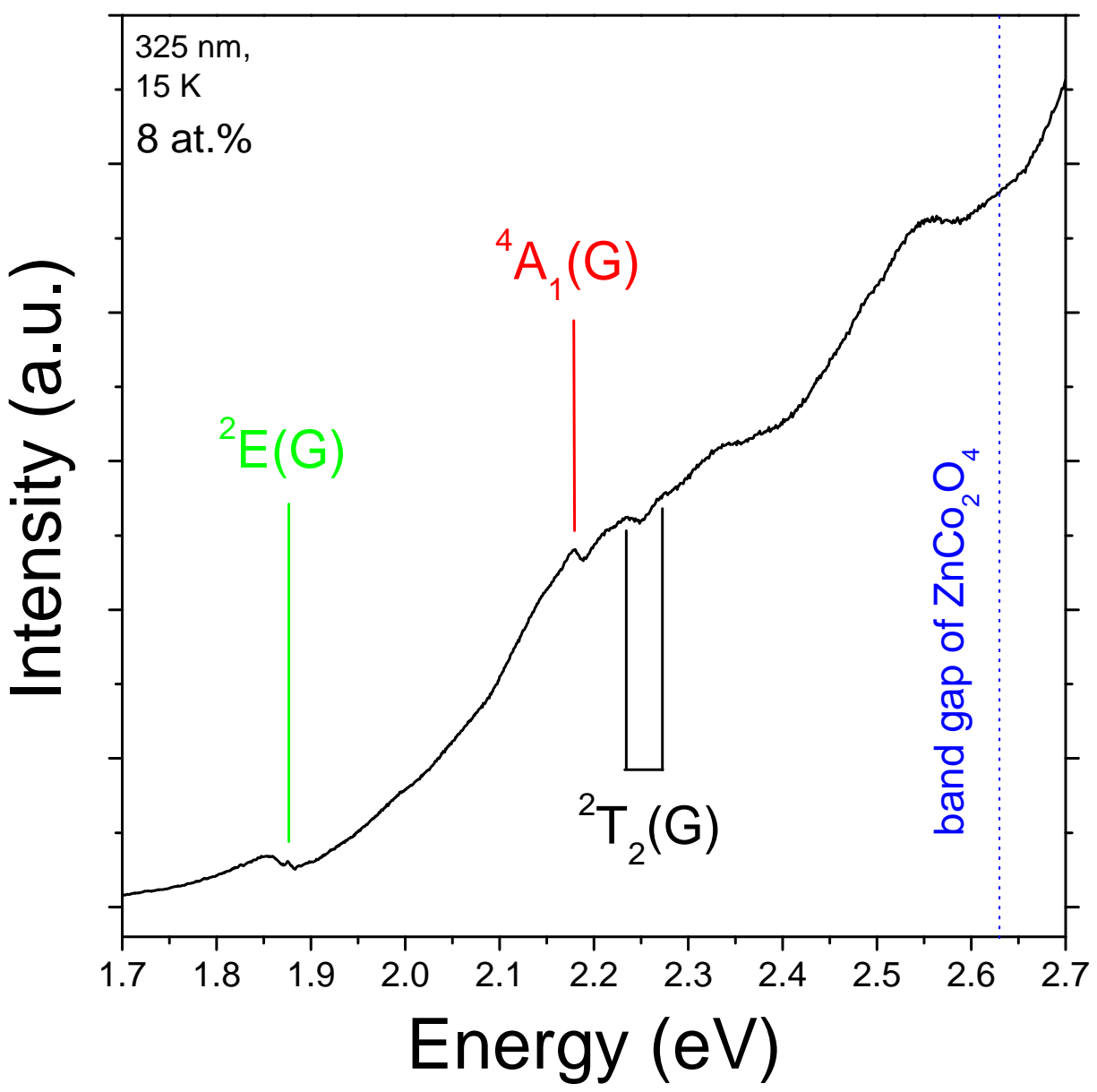

Figure 6.14: The 8 at.\% Co implanted $\mathrm{ZnO}$ single crystals shows some features at about $2.17 \mathrm{eV}$; $2.23 \mathrm{eV}$; and $2.26 \mathrm{eV}$, which could be assigned to higher 3d-shell states. The blue dotted line indicates the band gap of $\mathrm{ZnCo}_{2} \mathrm{O}_{4}$ at $2.63 \mathrm{eV}$ [402].

environment of the Co impurity and results in a stronger phonon coupling. This decrease of the zero phonon line is visible in the $\mathrm{Co}^{2+}$ intra-shell luminescence of the $\mathrm{ZnO}$ nanowires in figure 6.13a. This effect is not so strong in the Co implanted $\mathrm{ZnO}$ single crystals in figure $6.13 \mathrm{~b}$, but it is necessary to note, that the shown spectra of the Co implanted single crystals were recorded after annealing at $900{ }^{\circ} \mathrm{C}$. After annealing at $700{ }^{\circ} \mathrm{C}$ most transition metal implanted $\mathrm{ZnO}$ single crystals show extremely weak luminescence over the entire spectra. It might be possible that the intra-shell luminescence band of the $\mathrm{Co}^{2+}$ ion in $\mathrm{ZnO}$ nanowires could be improved by annealing at higher temperatures.

Almost all samples show no transitions from higher $\mathrm{Co}^{2+}$ intra shell states than the ${ }^{2} E(G)$ components. ${ }^{4} A_{1}(G)$ and ${ }^{2} T_{2}(G)$ components should appear on the low 
energy tail of the green luminescence band. Figure 6.14 shows the luminescence spectra of the 8 at.\% Co implanted $\mathrm{ZnO}$ single crystal recorded after annealing at $900{ }^{\circ} \mathrm{C}$. In figure 6.14 very weak and undefined transitions could be observed at about $2.17 \mathrm{eV} ; 2.23 \mathrm{eV}$; and $2.26 \mathrm{eV}$. These positions fit very well to luminescence transitions from ${ }^{4} A_{1}(G)$ and ${ }^{2} T_{2}(G)$ to the ground state ${ }^{4} A_{2}(F)$. However, samples with Co concentrations above 4 at.\% indicate the formation of $\mathrm{ZnCo}_{2} \mathrm{O}_{4}$ (see section 5.5.2), which is a semiconductor with a band gap of about $2.63 \mathrm{eV}$ [402]. The blue dotted line in figure 6.14 indicates the band gap of $\mathrm{ZnCo}_{2} \mathrm{O}_{4}$. Therefore, it is not possible to assigne the ${ }^{4} A_{1}(G)$ and ${ }^{2} T_{2}(G)$ transitions to the weak lines at $2.17 \mathrm{eV}$; $2.23 \mathrm{eV}$; and $2.26 \mathrm{eV}$, because it may be possible that those lines are transitions of $\mathrm{ZnCo}_{2} \mathrm{O}_{4}$. To my best knowledge, up to now no luminescence of $\mathrm{ZnCo}_{2} \mathrm{O}_{4}$ was reported.

\subsection{2 $\mathrm{Fe}^{3+}$ ion}

Iron ions are typically incorporated in $\mathrm{ZnO}$ with two different charge states [403]: $\mathrm{Fe}^{2+}$ and $\mathrm{Fe}^{3+}$. The $\mathrm{Fe}^{2+}$ ion has a electronic $d^{6}$ configuration with the free ion ground state ${ }^{5} D$ (see table 2.1), which is degenerated by tetragonal crystal field interaction into the ${ }^{5} E$ ground state term and the ${ }^{5} T_{2}$ excited state term $[23 ; 404 ; 405]$. The ${ }^{5} E$ ground state term and the ${ }^{5} T_{2}$ excited state term are further degenerated by spinorbit interaction and trigonal crystal field interaction into at least seven sub-levels $[23 ; 404]$. By absorption measurements the transition from the ground state ${ }^{5} E$ term to the ${ }^{5} T_{2}$ excited state term was observed in the far infra-red at about $400 \mathrm{meV}$ $[23 ; 404]$. Due to the fact that this optical transition is outside of our luminescence sensitivity range, the optical properties of the $\mathrm{Fe}^{2+}$ ions are not discussed any further.

The $\mathrm{Fe}^{3+}$ free ion has a electronic $d^{5}$ configuration with a ${ }^{6} S$ ground state and a ${ }^{4} G$ first excited state $[23 ; 25]$. The free ion states of the $\mathrm{Fe}^{3+}$ ion are degenerated in $\mathrm{ZnO}$ by crystal field perturbation (tetragonal as well as trigonal field components), spin-orbit interaction and Jahn-Teller effect. Figure 6.15 presents the degeneration of the $\mathrm{Fe}^{3+}$ ion. The tetrahedral crystal field transforms the ground state into a ${ }^{6} A_{1}(S)$ state, where the first excited state is fourfold degenerated with the ${ }^{4} T_{1}(G)$ term as the lowest state [23;24]. Spin-spin and second order spin-orbit interaction split the ${ }^{6} A_{1}(S)$ ground state into two states with $\Gamma_{7}$ and $\Gamma_{8}$ symmetry [23; 24], whereby the correct energetic order of these two states is up to now unknown. The axial distortion of the crystal field in hexagonal crystals with $C_{3 \nu}$ symmetry leads to a further split of the ground state ${ }^{6} A_{1}(S)$ levels into three Kramers doulbets [23; 24], which are separated by a few tens of $\mu \mathrm{eV}$. 


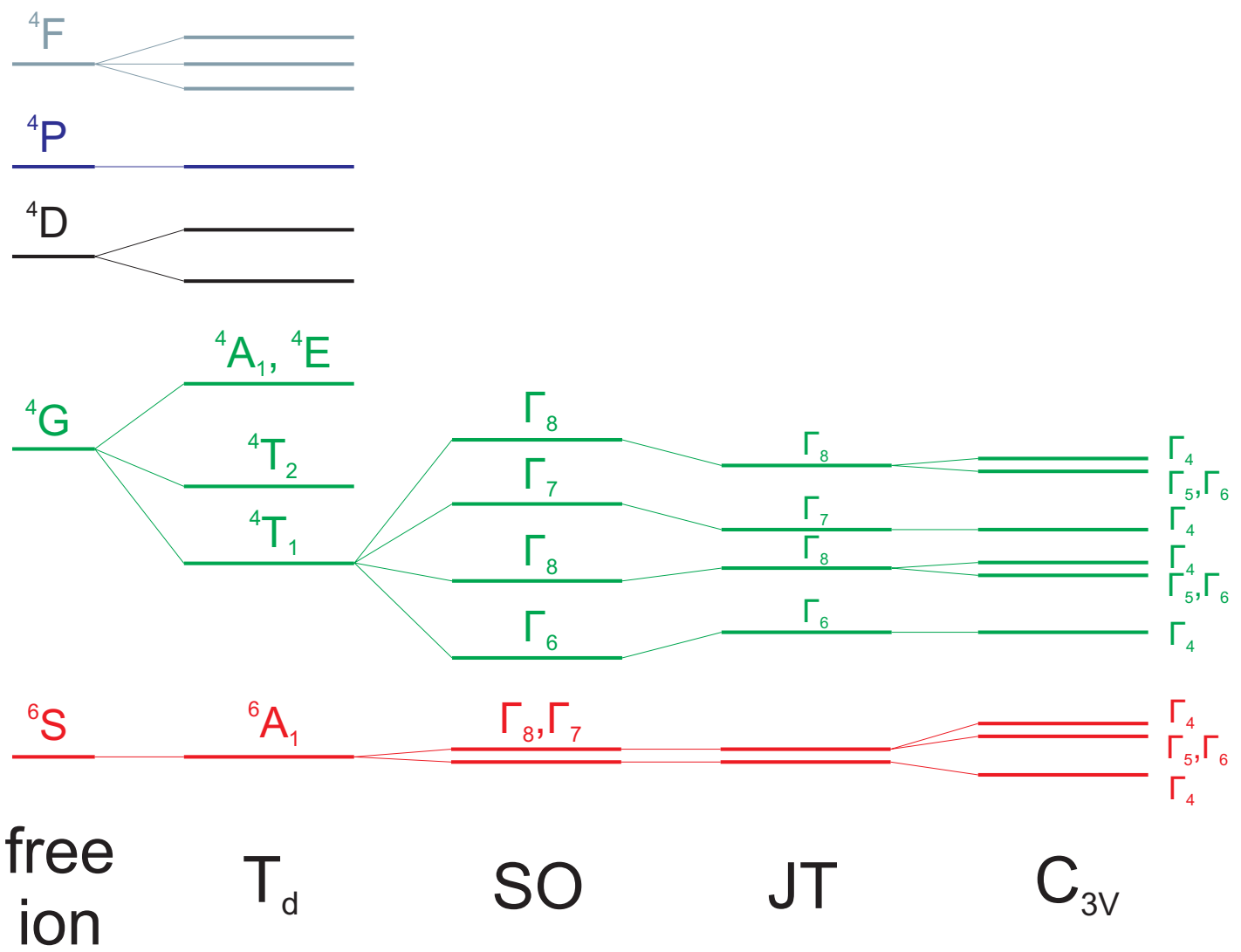

Figure 6.15: Term scheme of the $\mathrm{Fe}^{3+}$ ion in trigonal crystal fields such as $\mathrm{ZnO}$ : The free ion with electronic $d^{5}$ configuration is degenerated by tetragonal $\left(T_{d}\right)$ crystal field interaction; spin-orbit (SO) interaction; Jahn-Teller (JT) interaction; and trigonal $\left(C_{3 \nu}\right)$ crystal field interaction (adapted from [23; 25]).

The temperature dependent photoluminescence study of the $\mathrm{Fe}^{3+}$ luminescence band shows 'hot lines' on the high energy side [23; 24], which represent the fine structure of the first exited ${ }^{4} T_{1}(G)$ term. The ${ }^{4} T_{1}(G)$ state is split by further spin-orbit interaction, spin-spin interaction, trigonal crystal field and Jahn-Teller interaction into at least five states with a fine structure of a few meV [24]. The ${ }^{4} T_{1}(G)$ term is fourfold degenerated by spin-orbit interaction [23]. However, in cubic II-VI and III-V semiconductors it was found that those four sub-levels are reduced to two or three sub-levels by a dynamic Jahn-Teller distortion [23]. In trigonal symmetry, such as $\mathrm{ZnO}$, the lower symmetry of the crystal field reduces the Jahn-Teller distortion of the $\mathrm{Fe}^{3+}$ center [23]. Therefore, only an intermediate dynamic Jahn-Teller distortion reduces the fourfold spin-orbit splitting to about $4 \mathrm{meV}$ [23]. The trigonal crystal field with $C_{3 \nu}$ symmetry splits the four sub levels into six states with $\Gamma_{4} ; \Gamma_{5} ;$ and $\Gamma_{6}$ symmetry [23], which are only been resolved in $\mathrm{ZnO}$ and $\mathrm{GaN}$. 

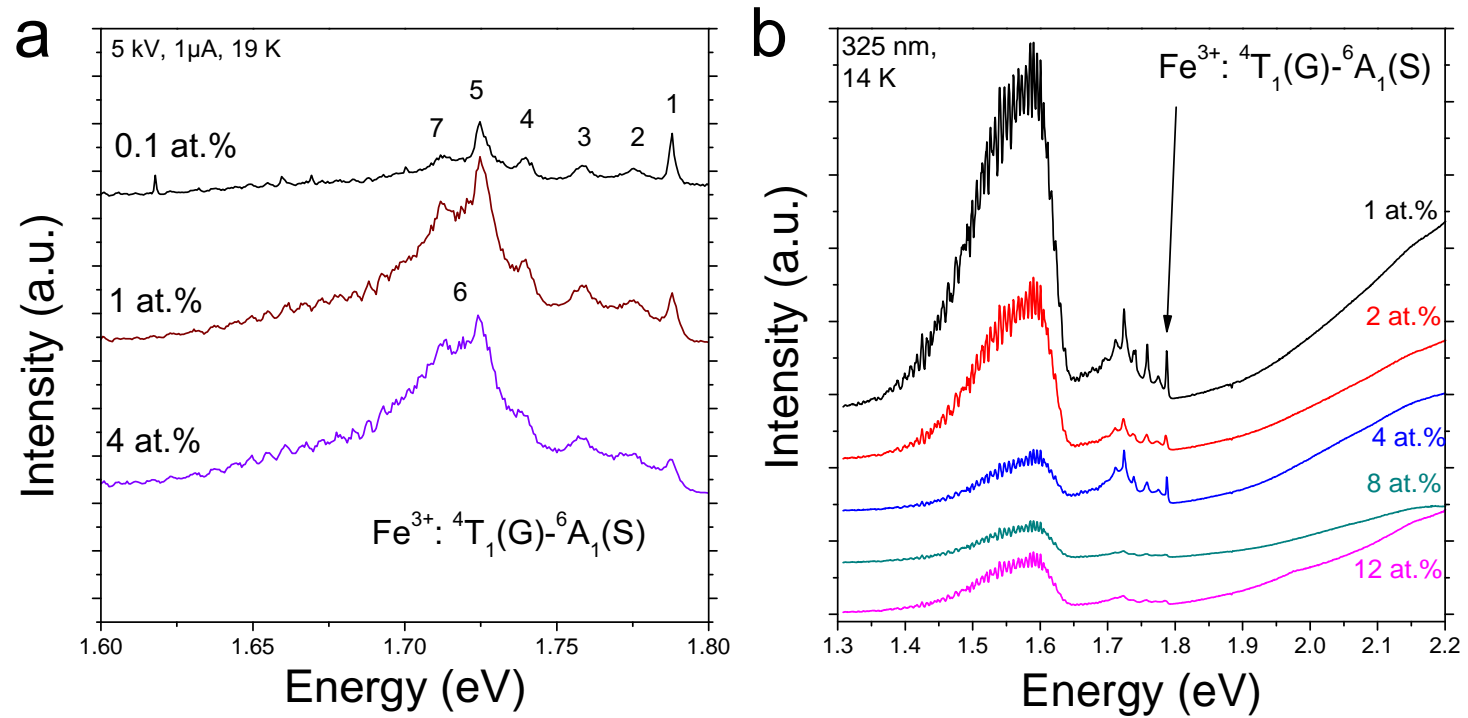

Figure 6.16: Cathodoluminescence spectra of Fe implanted ZnO nanowires show intra shell transitions of the $\mathrm{Fe}^{3+}$ ion at $19 \mathrm{~K}$. At $1.788 \mathrm{eV}$ a direct intra shell transition of $\mathrm{Fe}^{3+}$ from the ${ }^{4} T_{1}(G)$ excited state to the ${ }^{6} A_{1}(S)$ ground state is visible with a phonon side band on the low energy side.

Figure 6.16 shows sharp and comprehensive luminescence lines between 1.8 and $1.7 \mathrm{eV}$ of Fe implanted $\mathrm{ZnO}$ nanowires in (a) and for CrysTec single crystals in (b) at low temperature as function of the Fe concentration. For low Fe concentrations, this luminescence band is dominated by two sharp transitions at $1.788 \mathrm{eV}$ (line \#1) and $1.725 \mathrm{eV}$ (line \#5) in Fe implanted $\mathrm{ZnO}$ nanowires, where the relative intensity of the first one decreases with increasing Fe concentration compared to the second one. At the same time the broad transition at $1.713 \mathrm{eV}$ (line \#7) increases in intensity. However, the Fe implanted $\mathrm{ZnO}$ single crystals show no such concentration dependence like the nanowire sample, but it was necessary to anneal the implanted single crystals at higher temperature $\left(900{ }^{\circ} \mathrm{C}\right)$ to observe any luminescence. All Fe implanted CrysTec single crystals show a broad and strong band between 1.5 and $1.6 \mathrm{eV}$ (see figure 6.16), which has a higher intensity than all sharp lines at higher transition energies. This band is also visible in Fe implanted $\mathrm{ZnO}$ nanowires, but it is very weak (see figure 6.17).

The direct luminescence transition from the excited ${ }^{4} T_{1}(G)$ term to the ${ }^{6} A_{1}(S)$ ground state has an energy of $1.788 \mathrm{eV}$ and can be assigned to the line \#1 in figure 6.16. The splitting of the exited state ${ }^{4} T_{1}(G)$ as well as the ground state ${ }^{6} A_{1}(S)$ is about $4.4 \mathrm{meV}$ and $43 \mu \mathrm{eV}$ between the lowest and highest level, respectively. Table 6.4 specifies the spectral positions of all lines indicated in figure $6.16 \mathrm{a}$, whereby 
the direct transition from the ${ }^{4} T_{1}(G)$ term to the ${ }^{6} A_{1}(S)$ term is indicated as zero phonon line (ZPL). The spectral difference $\Delta E$ from the zero phonon line is greater than the fine splitting of both states (see table 6.4). Therefore, transitions at lower energies could not be assigned to a direct intra-shell transition of the $\mathrm{Fe}^{3+}$ center. The structured band on the low energy side of the direct intra-shell transition could be assigned to a phonon assisted side band, the spectral shift is used to assigne possible phonon modes with respect to the references [88; 303]. However, as discussed for intra-shell luminescence of the $\mathrm{Co}^{2+}$ ion, the phonon assignment in table 6.4 should be treated with attention.

The broad band between 1.5 and $1.6 \mathrm{eV}$ in Figure 6.16 can not be assigned to an intra-shell transition of the $\mathrm{Fe}^{3+}$ or to the phonon side band. This band is only observable in Fe implanted samples. Hence, a more complex defect containing Fe could be the origin for this luminescence band. On the other hand, all samples, which are implanted with at least 8 at.\% Fe, show the formation of secondary phases such as $\mathrm{ZnFe}_{2} \mathrm{O}_{4}$ and $\mathrm{Fe}_{2} \mathrm{O}_{3}$ (see section 5.5.3). Valenzuela et al. determine the band gap of both secondary phases to 1.92 and $1.82 \mathrm{eV}$ for $\mathrm{ZnFe}_{2} \mathrm{O}_{4}$ and $\mathrm{Fe}_{2} \mathrm{O}_{3}$ [406], respectively. Due to the fact, that up to now no luminescence properties of both phase were reported, we could not rule out a contribution of those secondary phases. However, all data from Raman, XRD, and TEM for low dose Fe implanted $\mathrm{ZnO}$ present no signal of any secondary phases, making the assignment to secondary phase luminescence unlikely.

Figure 6.17 shows temperature dependent photoluminescence spectra of the $\mathrm{Fe}^{3+}$

Table 6.4: Phonon side bands of the $F e^{3+}:{ }^{4} T_{1}(G)-{ }^{6} A_{1}(S)$ transition: spectral position $E$, difference to the zero phonon line (ZPL) $\Delta E$ and possible phonon modes of $\mathrm{ZnO}$ [88; 303].

\begin{tabular}{lrrr}
\hline Peak \# & $\begin{array}{r}\text { Energie } \\
(\mathrm{eV})\end{array}$ & $\begin{array}{r}\Delta E \\
(\mathrm{meV})\end{array}$ & $\begin{array}{r}\text { Identification } \\
\mathrm{ZPL}\end{array}$ \\
\hline 1 & 1.7882 & & $E_{2}^{\text {low }}[\Gamma]$ \\
2 & 1.7759 & 12.3 & $T O[\Gamma], L O[\Gamma]$ \\
3 & 1.7589 & 29.3 & $L O[\Gamma]$ \\
4 & 1.7397 & 48.5 & $A_{1}(T O)[\Gamma], E_{1}(T O)[\Gamma]$ \\
5 & 1.7252 & 63.0 & $A_{1}(L O)[\Gamma]$ \\
6 & 1.7166 & 71.6 & $T A+T O[H, M]$ \\
7 & 1.7117 & 76.5 &
\end{tabular}




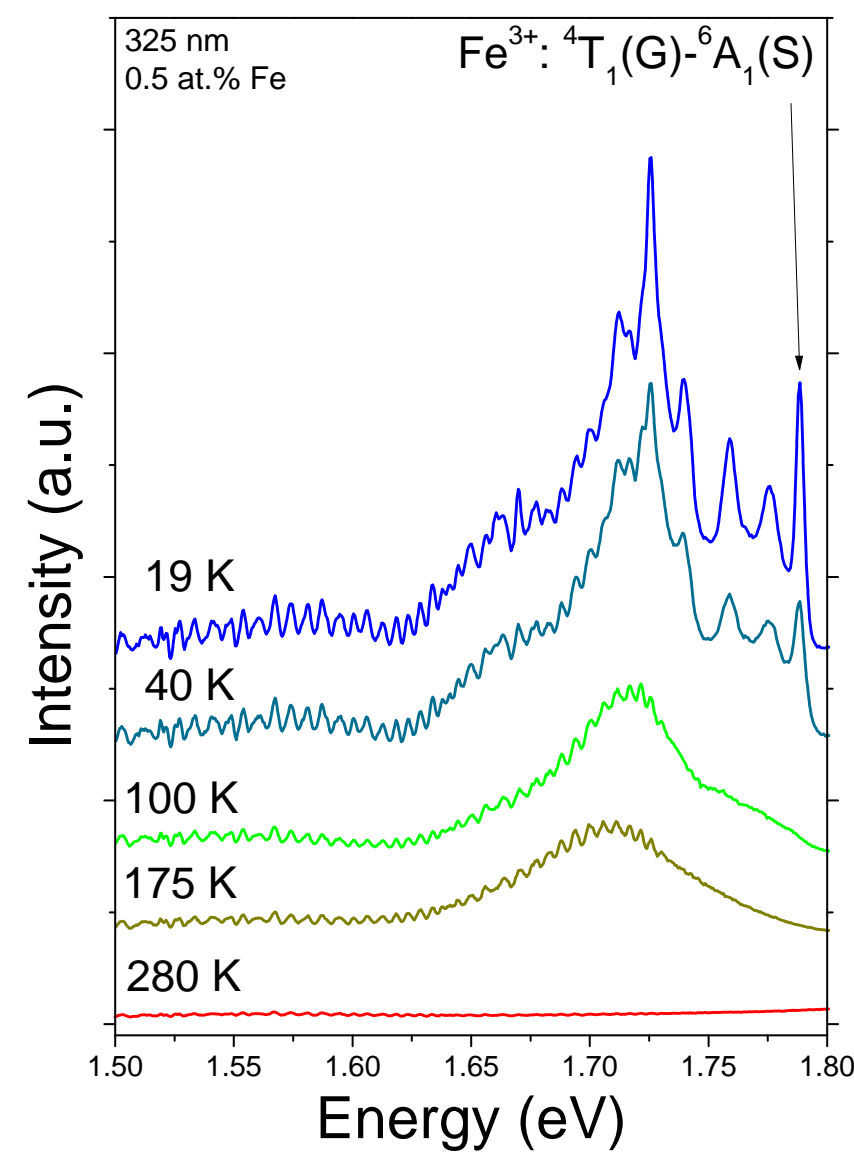

Figure 6.17: $\mathrm{Fe}^{3+}$ intrashell photoluminescence of 0.5 at.\% Fe implanted $\mathrm{ZnO}$ nanowires as function of measurement temperature [49]. At $19 \mathrm{~K}$ strong luminescence of the direct $\mathrm{Fe}^{3+}$ intra shell luminescence $(1.788 \mathrm{eV})$ and phonon assisted transitions could be observed, which changes drastically with increasing temperature and gets more dominated by phonon assisted side band transitions. No intra shell luminescence could be observed at room temperature.

intra shell luminescence of the 0.5 at.\% Fe implanted $\mathrm{ZnO}$ nanowires. With increasing temperature the relative intensity of the direct intra-shell luminescence decreases compared to the phonon side band, and could not be observed for temperatures above $100 \mathrm{~K}$. At $100 \mathrm{~K}$ only a broad phonon assisted transition could be observed, where a further increase in temperature leads to decreasing intensity. At room temperature no intra shell related luminescence could be observed at all. For rare earth implanted $\mathrm{ZnO}$ nanowires, room temperature intra-shell luminescence could be observed $[407 ; 408]$. However, in contrast to $4 f$ centers, the electron-phonon coupling is stronger in $3 d$ elements [126]. Therefore, it might be possible that the generation of phonons increases with increasing sample temperature and results in a strong quenching of the $3 d$ intra shell luminescence. This conclusion is supported by the remaining high defect density in our ion implanted $\mathrm{ZnO}$ nanowires after annealing. Further annealing at higher temperatures may reduce this effect. However, the influence of the excitation process of the $3 d$ centers must be discussed at this point, too. Below $77 \mathrm{~K}$ the presence of Fe3+ centers was not observed by ESR measurements 
and therefore the $\mathrm{Fe} 2+$ center is the unexcited charge state at low temperatures $[24 ; 403]$. The absence of a charge transfer level from $\mathrm{Fe}^{2+}$ to $\mathrm{Fe}^{3+}$ within the $\mathrm{ZnO}$ band gap shows the complexity of the excitation processes [23]. The luminescence of $\mathrm{Fe}^{3+}$ is excited by an energy transfer process from a deep acceptor to the $\mathrm{Fe}^{2+}$ center by photogenerated holes, where the nature of the acceptor is up to now unknown [24]:

$$
\begin{aligned}
F e^{2+}+A^{0}+\hbar \omega_{\text {blue }} & \rightarrow F e^{2+}+A^{-}+h_{v b} \\
& \rightarrow F e^{3+}\left({ }^{4} T_{1}(G)\right)+A^{-} \\
& \rightarrow F e^{3+}\left({ }^{6} A_{1}(S)\right)+A^{-}+\hbar \omega_{\text {red }} .
\end{aligned}
$$

The excitation with light transfers an electron to the neutral acceptor leaving a hole in the valance band, which is transfered to the $\mathrm{Fe}^{2+}$ ion. This hole transfer leads to a charge transfer from the $\mathrm{Fe}^{2+}$ ion to an excited $\mathrm{Fe}^{3+}$ ion. The intensity as well as the temperature dependence of the $\mathrm{Fe}^{3+}$ intra-shell luminescence depends strongly on the nature and the temperature behavior of the deep acceptor.

Figure 6.18 a shows the power dependence of the $\mathrm{Fe}^{3+}$ intra-shell photoluminescence of 0.5 at.\% Fe implanted $\mathrm{ZnO}$ nanowires at low temperatures. A decrease in excitation power does not effect the intensity ratios between those lines. Figure $6.18 \mathrm{~b}$ shows a double logarithmic plot of the integrated intensity ${ }^{4}$ as function of the excitation power. For help two linear slops are ploted within the diagram with $m=1$ (linear intensity increase) and $m=2$ (quadratic intensity increase). The intensity of the intra-shell luminescence shows a almost linear gain. But, for 'high' excitation power the integrated intensity of the $\mathrm{Fe}^{3+}$ intra-shell photoluminescence veers away from the linear behavior and shows a stronger rise in intensity. This behaviour suggests a transfer from spontaneous emission to stimulated emission with a minimal trashold value of $\sim 300 \mathrm{~mW} / \mathrm{cm}^{2}$. However, thresholds for stimulated emission in $\mathrm{ZnO}$ nanowires for band to band transitions has been reported between $8-867 \mathrm{~kW} / \mathrm{cm}^{2}[37 ; 409 ; 410]$, which is about five orders of magnitude higher. The band to band stimulated emission can be achieved by exciton-exciton scattering or electron-hole plasma recombination [409], which shows a much faster decay time of stimulated emission than the decay time of spontaneous emission. Therefore, if the above presented over-linear intensity increase of the $\mathrm{Fe}^{3+}$ luminescence is due to a transition into stimulated emission, the decay time for 'high' excitation power should be lower than the decay time of $25 \mathrm{~ms}$ for $\mathrm{Fe}^{3+}$ luminescence [25].

On the other hand, effects of impurity traps; excitation and deexcitation paths for

\footnotetext{
${ }^{4}$ The integrated intensity is determined by subtracting the background of the green luminescence band and integration over the entire $\mathrm{Fe}^{3+}$ band.
} 

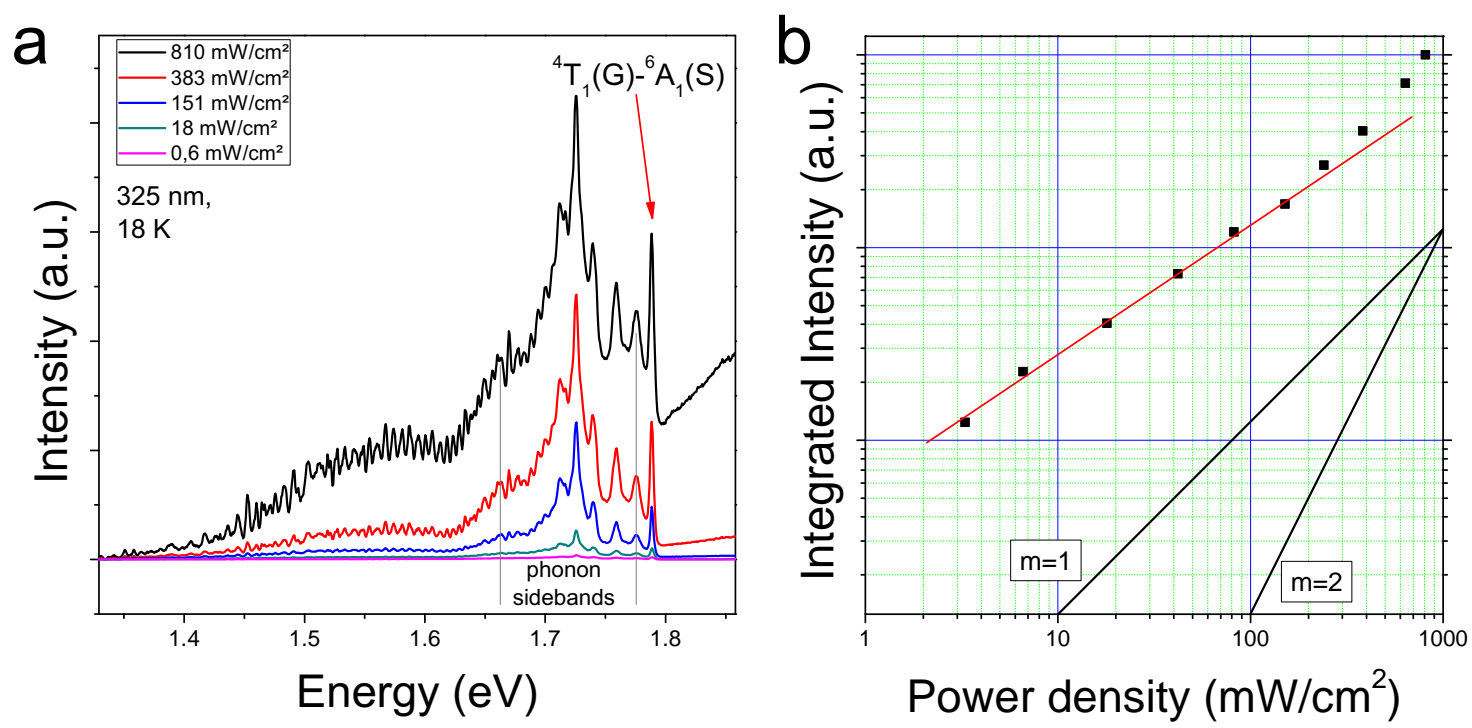

Figure 6.18: Power dependent photoluminescence of the $\mathrm{Fe}^{3+}$ intra-shell luminescence transition is shown for variation of the excitation power of the HeCd-laser in (a) of the 0.5 at.\% Fe implanted ZnO nanowires. (b) shows the double logarithmic plot of the integrated intensity as function of the excitation power of the $\mathrm{Fe}^{3+}$ intra-shell luminescence from (a).

intra-shell luminescence; and other direct or indirect involved luminescence channels must be discussed. For intra-shell luminescence several excitation paths are possible [411]: resonant absorption of a photon; transitions at impurities (for example donor acceptor pairs); or capture of an electron-hole pair. The $\mathrm{Fe}^{3+}$ intra-shell luminescence (see 6.3) is excited via an unknown acceptor [24]. This acceptor could have a low capture cross section for free carriers or take part in secondary luminescence channels, which could be preferred over the energy transfer to the Fe center. With increasing excitation power impurities with higher capture cross section for free carriers as well as possible secondary luminescence channels would be saturated, resulting in an increasing energy transfer channel to the Fe center above the threshold value. However, nonradiative recombination channels should show an effect onto the power dependent photoluminescence properties of the Fe center as well. In addition to radiative deexcitation, nonradiative deexcitation channels could be saturated with increasing excitation power. Such nonradiative deexcitation channels are [411]: auger deexcitation, multiphonon deexcitation, or transfer to killer centers.

Figure 6.19 shows the rather low intensity of the $\mathrm{Fe}^{3+}$ intra-shell luminescence for excitation power of $0.8 \mathrm{~W} / \mathrm{cm}^{2}$ compared to the dominating structured green band. This situation changes completely under excitation with the high power Nd:YAG at 


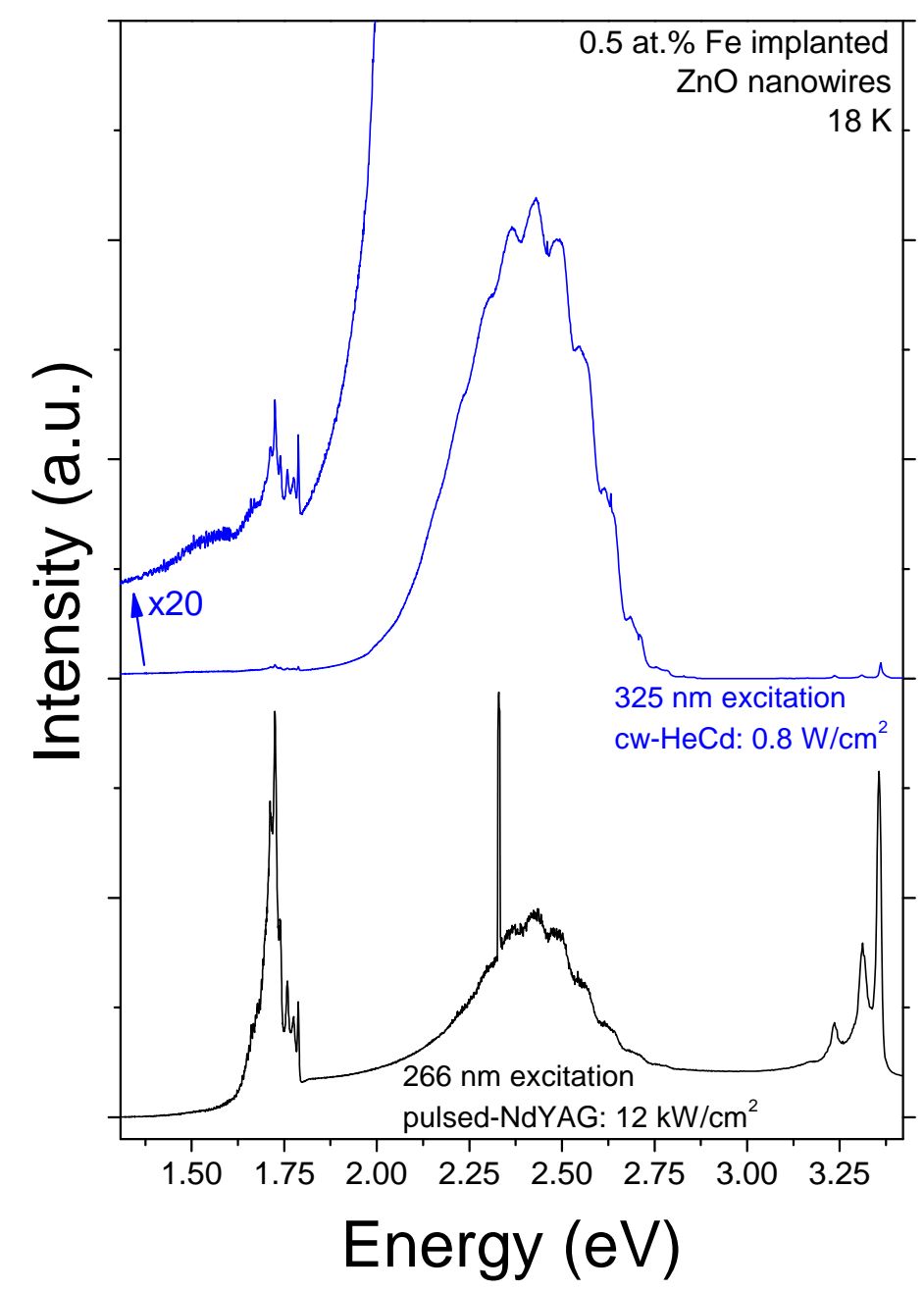

Figure 6.19: The comparison between 'high power' excitation with $266 \mathrm{~nm} \quad \mathrm{Nd}: Y A G$-laser line and 'low power' with $325 \mathrm{~nm}$ HeCd-laser line. The sharp line on the structured green luminescence band is the $532 \mathrm{~nm}$ $N d: Y A G$ laser line.

$12 \mathrm{~kW} / \mathrm{cm}^{2}$. The $\mathrm{Fe}^{3+}$ intra-shell luminescence transition as well as the near band gap luminescence show the highest peak intensity under excitation with $12 \mathrm{~kW} / \mathrm{cm}^{2}$, whereby both play only minor role under 'low power' excitation. The most interesting point of figure 6.19 is the dramatic increase of the $\mathrm{Fe}^{3+}$ intra-shell luminescence with the drastic increase of excitation power. It would be interesting to see the variation of integrated intensity of the intra-shell luminescence compared to the green luminescence band with increasing excitation power in the range from $0.8 \mathrm{~W} / \mathrm{cm}^{2}$ to $12 \mathrm{~kW} / \mathrm{cm}^{2}$. The intensity ratio $I_{\text {green }} / I_{\mathrm{Fe}^{3+}}$ between the green luminescence and the intra-shell luminescence is $\sim 66$ for excitation with $0.8 \mathrm{~W} / \mathrm{cm}^{2}$ and $\sim 0.5$ for $12 \mathrm{~kW} / \mathrm{cm}^{2}$. However, the decrease of the intensity ratio $I_{\text {green }} / I_{\mathrm{Fe}^{3+}}$ is only about two orders of magnitude with increasing excitation power of six orders of magnitude. The approximation of the intensity ratio $I_{\text {green }} / I_{F e^{3+}}$ neglects the intensity increase of both luminescence bands with increasing excitation power, which could 


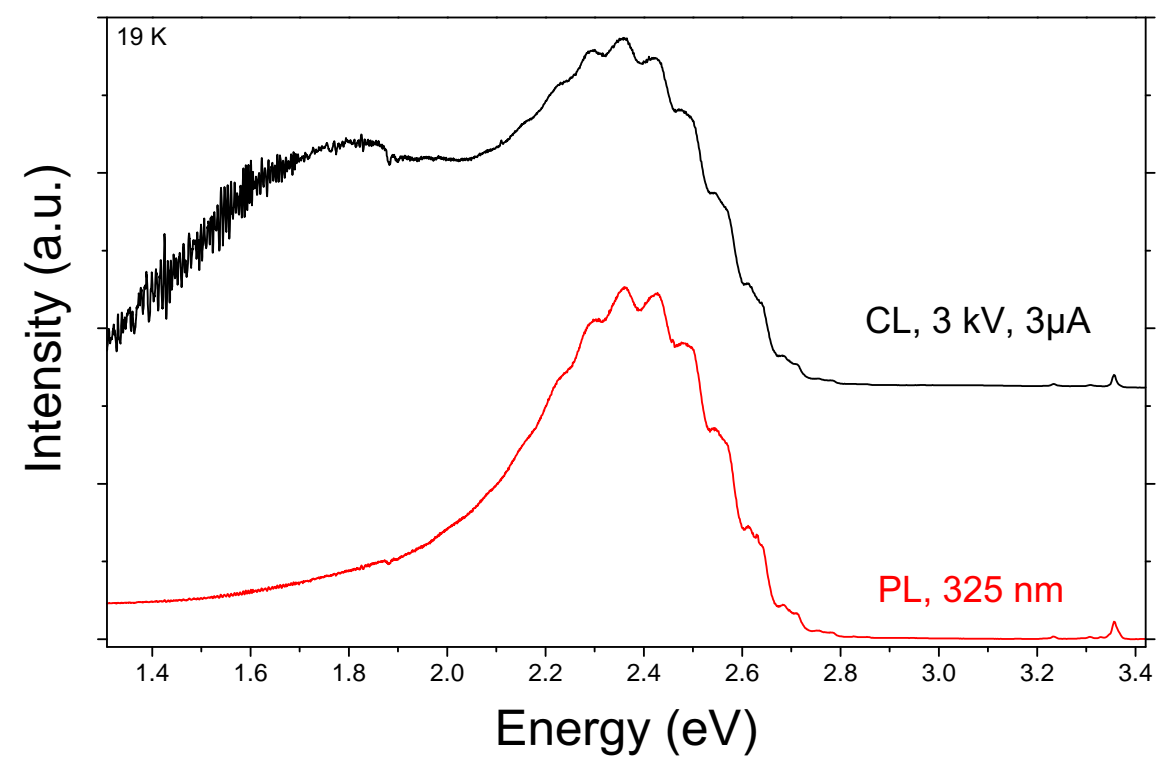

Figure 6.20: Cathodoluminescence and Photoluminescence of 1 at.\% Ni implanted $\mathrm{ZnO}$ nanowires.

not simply determined due to different optical adjustments of the PL setup. For future measurements it would be interesting to determine the variation of the intensity ratio between both luminescence bands as well as the variation of the integrated intensity for every luminescence band as function of the excitation power.

\subsection{3 $\mathrm{Ni}$ and $\mathrm{Mn}$ ions in $\mathrm{ZnO}$}

Figure 6.20 shows the photoluminescence and cathodoluminescence of the 1 at. $\% \mathrm{Ni}$ implanted $\mathrm{ZnO}$ nanowires, which displays only a weak unstructured band at around $1.8 \mathrm{eV}$ for excitation with the electron beam. All photoluminescence spectra of $\mathrm{Ni}$ implanted $\mathrm{ZnO}$ do not show this band, no matter which laser line was used for excitation. Nickel is typically a double positive charged ion $\mathrm{Ni}^{2+}$, if it is incorporated on a zinc lattice side and has therefore a $d^{8}$ configuration [22; 412]. The free $\mathrm{Ni}^{2+}$ ion has a ${ }^{3} \mathrm{~F}$ ground state, which is threefold degenerated by the tetragonal crystal field $[22 ; 412]$. These levels are further degenerated by spin-orbit interaction as well as the trigonal crystal field. However, optical transitions between those states were observed by absroption measurements in the far infra-red spectral region $[22 ; 128$; 412]. Weakliem and Kaufmann et al. have observed by absortion measurements transitions from the $\mathrm{Ni}^{2+}$ ion ground state to the degenerated levels of the $\mathrm{Ni}^{2+}$ free ion states ${ }^{1} D$ and ${ }^{3} P$ in the near infra-red and visible spectral region [22; 128]. The 
reported spectral position of the $T_{2}-{ }^{3} T_{1}(P)$ spin-orbital term at about $1.885 \mathrm{eV}$ fits very well to the onset of additional luminescence bands in $\mathrm{Ni}$ implanted $\mathrm{ZnO}$ in figure $6.20[22 ; 128]$. The $T_{2}$ state is the lowest spin-obital degenerated state of the ${ }^{3} T_{1}(P)$ tetragonal crystal field transformed ${ }^{3} \mathrm{P} \mathrm{Ni}^{2+}$ free ion state $[22 ; 128]$. Though, the onset of the $\mathrm{Ni}$ band is shifted to lower energies than the reported spectral position of the direct transition. Therefore, it could be assumed that the additional band in figure 6.20 might be the phonon side band of the $\mathrm{Ni}^{2+}$ cathodoluminescence, but a clear assignment is not suitable due to the absence of the direct $T_{2}-{ }^{3} T_{1}(P)$ luminescence of the $\mathrm{Ni}^{2+}$ ion.

Absorption measurements of $\mathrm{Ni}$ doped $\mathrm{ZnO}$ by Weakliem show an additional charge transfer band with an onset at about $2.6 \mathrm{eV}$ and ranges up to the band gap of $\mathrm{ZnO}$ [22]. Excitation of the $\mathrm{Ni}$ doped $\mathrm{ZnO}$ with photon energies around the band gap lead to a charge transfer process of the $\mathrm{Ni}^{2+}$ ion to the $\mathrm{Ni}^{3+}$ ion [398]. Latter one has an electronic $d^{7}$ configuration and intra-shell photoluminescence was observed at about $750 \mathrm{meV}$ [398]. However, cathodoluminescence could excite the $d$-shell by direct impact excitation [411], resulting in a small fraction of excited $\mathrm{Ni}^{2+}$ ions. This small fraction of $\mathrm{Ni}^{2+}$ ions could be the source of the additional luminescence band in Ni implanted $\mathrm{ZnO}$ samples. Direct excitation of the $d$-shell by photons is only possible, if the energy of the exciting photon matches the energy difference between the ground and excited state. Therefore, excitation with the HeCd laser line $(325 \mathrm{~nm})$ or Nd:YAG laser line $(266 \mathrm{~nm})$ lead to the charge transfer process and does not excite the $\mathrm{Ni}^{2+}$ ion.

In section 5.5.4 it was shown that $\mathrm{NiO}$ secondary phases are formed after annealing of high dose $\mathrm{Ni}$ implanted $\mathrm{ZnO}$ single crystals. $\mathrm{NiO}$ is a wide band gap semiconductor with an energy gap of about $4-4.3 \mathrm{eV}$ (see table 2.5). Cathodoluminescence studies of $\mathrm{NiO}$ show a broad band in the visible and near infra-red spectral region [131]. However, all high dose $\mathrm{Ni}$ implanted $\mathrm{ZnO}$ samples have really bad luminescence properties and show a broad band between 3.5 and $2 \mathrm{eV}$, which is a composition of the background glow; $\mathrm{ZnO}$ photoluminescence and possibly $\mathrm{NiO}$ luminescence. Therefore, a clear NiO luminescence could not be observed.

The $d$-shell configuration of manganese ions in $\mathrm{ZnO}$ are up to now not really understood, especially no intra-shell luminescence of $\mathrm{Mn}$ ions are reported in contrast to other semiconductor materials such as ZnS [235]. The charge state of manganese ions in $\mathrm{ZnO}$ was determined by electron paramagnetic resonance to be double positive charged $\mathrm{Mn}^{2+}$ with an electronic $d^{5}$ configuration [413; 414]. The configuration of the free ion orbitals of the $\mathrm{Mn}^{2+}$ ion as well as the crystal field degeneration should be comparable to the $\mathrm{Fe}^{3+}$ ion in $\mathrm{ZnO}$ (see figure 6.15), whereby the degeneration 
strength depends on the interaction of the Mn ion with the ligands. Bates et al. had presented absorption measurements of the $\mathrm{Mn}$ doped $\mathrm{ZnO}$ crystals with transition energies of about $1.88 \mathrm{eV} ; 2.95 \mathrm{eV}$; and $3.26 \mathrm{eV}$ [307], which are assigned to transitions from the $\mathrm{Mn}^{2+}$ ground state ${ }^{6} A_{1}(S)$ to ${ }^{4} T_{1}(G),{ }^{4} T_{2}(G)$, and ${ }^{4} A_{1} /{ }^{4} E(G)$ excited states, respectively. In contrast to this results Mizokawa et al. presented by cluster model calculated values for these transitions of [158]: $2.55 \mathrm{eV}$ for ${ }^{4} T_{1}(G), 2.85 \mathrm{eV}$ for

${ }^{4} T_{2}(G), 2.97 \mathrm{eV}$ for ${ }^{4} E(G)$, and $2.99 \mathrm{eV}$ for ${ }^{4} A_{1}(G)$. However, all photoluminescence and cathodoluminescence spectra of $\mathrm{Mn}$ implanted $\mathrm{ZnO}$ show no additional transition, which could be assigned to the theoretical values of Mizokawa et al. or to the absorption measurements of Bates et al.. Kleinlein and Helbig presented absorption measurements comparable to the results of Bates et al., but had assigned the absorption band below the band edge to a charge transfer region from the $\mathrm{Mn}^{2+}$ ion to either $\mathrm{Mn}^{+}\left(3 d^{6}\right)$ or $\mathrm{Mn}^{+}\left(3 d^{5} 4 s\right)$ [308].

\subsection{White color luminescence emission of $\mathrm{V}$ im- planted ZnO PLD films}

During the luminescence measurements of the $\mathrm{V}$ implanted $\mathrm{ZnO}$ PLD thin films a whitish luminescence of those samples was noticed. These whitish luminescence is illustrated in figure 6.21, the pictures are taken with a digital single lens reflex camera (Canon EOS 350D) under excitation with the $325 \mathrm{~nm}$ HeCd-laser line at room temperature (RT) and at $18.5 \mathrm{~K}$. All V implanted samples reveal a whitish luminescence in contrast to the Ar implanted ZnO PLD film, which shows a orange luminescence emission. As shown in reference [49] the 5 at.\% $\mathrm{V}$ implanted $\mathrm{ZnO}$ PLD thin film shows a 230 times higher total intensity at room temperature than the 0.8 at. $\% \mathrm{~V}$ implanted sample and a 325 times higher luminescence than the $\mathrm{Ar}$ implanted reference sample.

The color of the luminescence emission depends on the intensity of the deep luminescence bands shown in figure 6.7a. The physical and chemical nature of those bands was discussed before. The color of a light emitting diode is determined by the position in the CIE chromaticity diagram in figure $6.22 \mathrm{a}$, whereby the coordinates present a color by numbers and were determined via the CIE standard presented in section 4.5 by the equations 4.7 and 4.8. The results are shown in figure 6.22. The specific color regions blue, green, red and white are indicated in the diagrams in figure 6.22. In the detail diagrams the white region is marked with respect to reference [284]. 


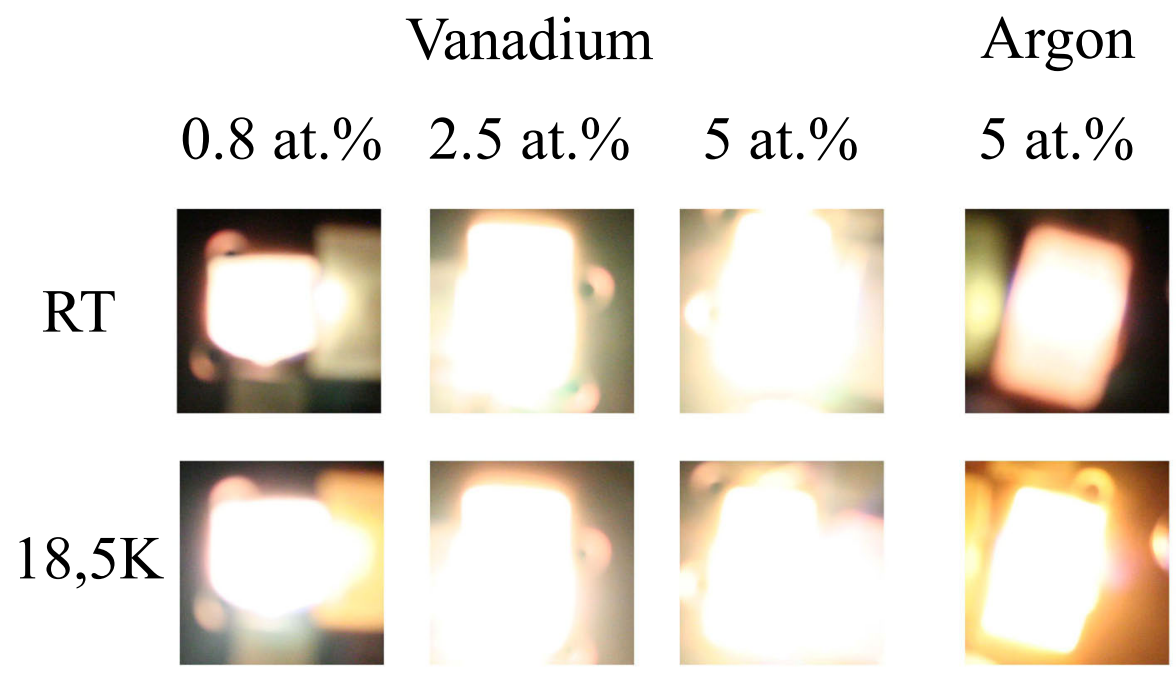

Figure 6.21: Pictures of photoluminescence emission of $V$ implanted ZnO PLD thin films at room temperature (RT) and $18.5 \mathrm{~K}$ show a whitish color except the Ar reference sample, which shows an orange luminescence emission. All pictures are taken with a digital single lens reflex camera (Canon EOS 350D) under excitation with the $325 \mathrm{~nm} \mathrm{HeCd}$-laser line.

The color of the visible luminescence band represented in dependence of vanadium concentration (figure 6.7a), temperature (figure 6.10) and excitation intensity (figure 6.11) is evaluated in CIE color space. The chromaticity diagram reveals an almost perfect white luminescence color for all $\mathrm{V}$-implanted $\mathrm{ZnO}$ films (see figure 6.22) [284], which confirms the visual impression (see figure 6.21). Furthermore, figures $6.22 \mathrm{~b}$ and $6.22 \mathrm{c}$ demonstrate that the color depends on $\mathrm{V}$ content, temperature, and excitation intensity. An increasing distance from the white color region can be observed with increasing vanadium concentration. Therefore, samples with less implanted V should be perfect white light emitters. The luminescence color becomes greener with increasing measuring temperature (not visible by eye), which is indicated by the red and blue arrow in figure $6.22 \mathrm{~b}$. This shift of the luminescence color is due to the diminishing red spectral region (see figure 6.10). The argon irradiated reference sample is located in the orange part of the chromaticity diagram and moves into the white color region with increasing measurement temperature due to the rising green luminescence in figure 6.8. Another parameter which may be used to control the color of visible luminescence is the excitation intensity. For example at low temperature the luminescence color in the Ar implanted $\mathrm{ZnO}$ and the 0.8 at.\% $\mathrm{V}$-implanted $\mathrm{ZnO}$ shifts from the reddish orange into orange and from orange into yellow (see figure 6.22c), respectively. 

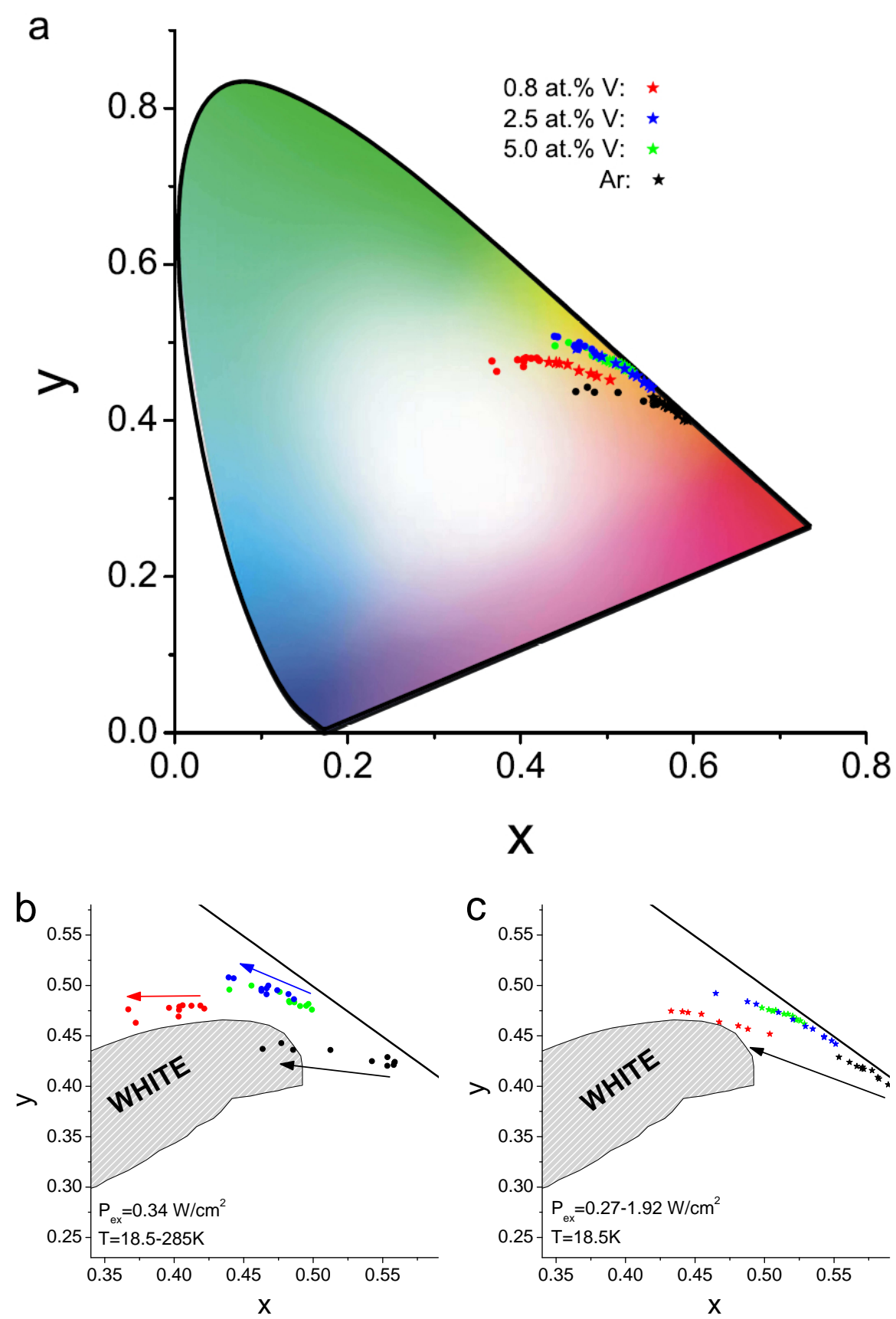

Figure 6.22: (a) gives an overview over chromaticity parameters in dependence on $V$ concentration, the temperature and excitation intensity. The temperature dependence and dependence on excitation intensity are given in detail c) and d) [49], whereas the arrows suggest the change of the chromaticity parameter with increasing temperature and excitation intensity, respectively. 


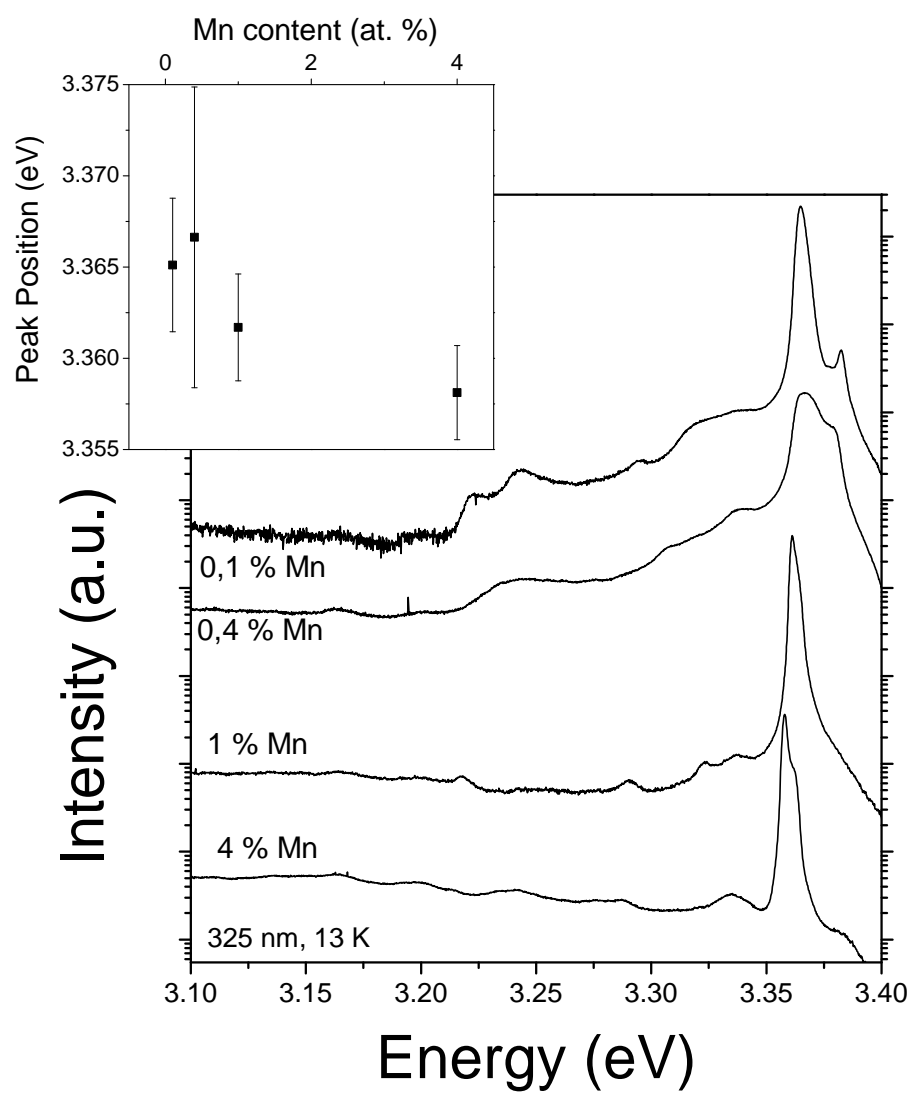

Figure 6.23: The near band gap luminescence of Mn implanted ZnO single crystals presents a shift of the luminescence peaks to lower energies. The inset shows the peak position as function of the Mn concentration.

\subsection{Band gap shift in $\mathrm{ZnO}: \mathrm{Mn}$}

As discussed in section 2.3, the alloying of $\mathrm{ZnO}$ with transition metals should have an effect on the band gap of the semiconductor. The size of the band gap should vary as function of the transition metal content between the band gaps of $\mathrm{ZnO}$ and the transition metal oxide (see section 2.3). Therefore, the band gap of Mn implanted $\mathrm{ZnO}$ samples should increase with increasing Mn concentration from the band gap of $\mathrm{ZnO}$ to the band gap of $\mathrm{MnO}$, which are found to be around $3.47 \mathrm{eV}$ and 3.6-3.8 eV $[1 ; 130]$, respectively. Figure 6.23 shows the near band gap photoluminescence of Mn implanted $\mathrm{ZnO}$ single crystals with increasing $\mathrm{Mn}$ concentration. At about $3.6 \mathrm{eV}$ a sharp and strong transition is observed, which shifts with increasing Mn concentration to lower peak positions. Commonly those luminescence transitions are labeled as donor bound exciton transitions (see beginning of this chapter 6 on page 105). 
The peak position was determined by fitting of Gaussian functions to the peaks in figure 6.23, the results are presented in the inset of figure 6.23. The peak position shifts about $7 \mathrm{meV}$ to lower energies, and is in contrast to the expectation of an increasing band gap. However, such a decrease in $\mathrm{Mn}$ alloyed $\mathrm{ZnO}$ was observed by Bhat et al. by absorption measurements on thin films. The bowing of the band gap with increasing transition metal content is attributed to the strong $s p-d$ interaction between the transition metal ions and the ligands (see section 2.3 and references $[133 ; 134])$.

Bhat et al. shows also a shift for $\mathrm{ZnCoO}$ and $\mathrm{ZnNiO}$, which is not unexpected due to the fact that $\mathrm{NiO}$ as well as $\mathrm{CoO}$ are semiconducting with band gaps of 4-4.3 $\mathrm{eV}$ and $\sim 3 \mathrm{eV}$ [130], respectively. However, due to extreme low luminescence intensity of all $\mathrm{Ni}$ and $\mathrm{Co}$ implanted $\mathrm{ZnO}$ single crystals and the superposition with background glow of the PL setup, it was not possible to determine the near band gap luminescence of those samples.

\subsection{Summary}

After ion implantation and annealing of the radioactive ${ }^{111} \mathrm{In}$ implanted $\mathrm{ZnO}$ sample the $\mathrm{I}_{9}$ luminescence line in the near band gap luminescence of $\mathrm{ZnO}$ appears. The decay time of the $\mathrm{I}_{9}$ line intensity corresponds to the half-live of the radioactive ${ }^{111}$ In isotope. By perturbed- $\gamma \gamma$-angular-correlation the lattice site of the implanted In was identified as the zinc lattice site in $\mathrm{ZnO}$ [75]. Due to this observation the $\mathrm{I}_{9}$ line was assigned to the shallow indium donor. The daughter product of ${ }^{111} \mathrm{In}{ }^{111} \mathrm{Cd}$ induces a broad band centered at about $2.85 \mathrm{eV}$, which might be induced due to lattice deformation or differences in electronegativity.

All transition metal implanted $\mathrm{ZnO}$ samples as well as all argon implanted $\mathrm{ZnO}$ samples show the generation of deep defect luminescence bands after annealing in air or oxygen ambient. These deep luminescence bands were divided into at least two different luminescence bands: a red-yellow luminescence band centered at about $1.9 \mathrm{eV}$ and a structured green luminescence band centered at about $2.4 \mathrm{eV}$. Both bands can not be assigned to oxygen vacancies due to the annealing in air or oxygen, which should reduce the concentration of oxygen vacancies. This conclusion is supported by the absence of the electron trap with an activation energy of $530 \mathrm{meV}$ DLTS measurements [382-384]. The red-yellow luminescence band is assigned to interstitial oxygen with tetrahedral surroundings, which are generated during the 
annealing in air or oxygen by two competing processes: recombination of ion implantation induced defects and oxidation.

Typically the green structured luminescence band was assigned to Co acceptors in $\mathrm{ZnO}$ [90; 351], but the drastic increase of the structured green luminescence band after ion implantation and annealing reveals a more complicated process behind this luminescence band. The configuration coordinate model was used to describe the broad as well as the fine structure of the green luminescence band. The structured green luminescence band was assigned to a luminescence transition from a shallow donor to a deep acceptor, which is further supported by the temperature dependent photoluminescence measurements. With increasing temperature the luminescence channel transforms from the donor-acceptor pair transition to a band to acceptor transition due to the ionization of the shallow donor. However, high resolution spectra reveal that two different shallow donors contribute to this deep luminescence band.

The nature behind the shallow donor and the deep acceptor was up to now unknown. Due to the appearance of the structured green luminescence band in all transition metal implanted $\mathrm{ZnO}$ samples, this luminescence band can not be assigned to defects involving the implanted transition metal elements. However, the transition metal ion implantation into $\mathrm{ZnO}$ plays an important role during the generation of this defect. The transition metal ion implantation results in the generation of interstitial zinc, which introduces shallow donor levels into the band gap $[353 ; 354]$ and should give rise to the $I_{3,3 a}$ line [352]. But as no increase of the near band gap luminescence was observed, the structured green luminescence band dominates the luminescence of all transition metal implanted $\mathrm{ZnO}$ samples. Sun et al. assumed that the tetrahedral and octahedral zinc interstitials generate shallow donor levels $31 \mathrm{meV}$ and $61 \mathrm{meV}$ below the conduction band. The discrepancy between both levels fits very well to the fine structure separation of $30 \mathrm{meV}$, therefore it was assumed that the two interstitial zinc donors contribute to the structured green luminescence band. On the other hand, the octahedral oxygen interstitial is a deep acceptor, and introduces levels at $0.57 \mathrm{eV}$ and $1.18 \mathrm{eV}$ above the valence band depending on the charge state $[353 ; 354]$. Oxygen on antisides introduces deep acceptor levels between $0.283 \mathrm{eV}$ and $0.799 \mathrm{eV}$ above the valence band [400]. Therefore the structured green luminescence band was assigned to a transition from the two different interstitial zinc donors to interstitial oxygen on octahedral lattice sites or oxygen on antisite.

The combination of the red-yellow luminescence band and the structured green luminescence band results in a almost whitish luminescence emission of the vanadium implanted $\mathrm{ZnO}$ thin films, which offers the potential for white light emitting diodes. 
$\mathrm{Co}$ and Fe implanted $\mathrm{ZnO}$ samples show 'new' sharp luminescence transitions at $1.878 \mathrm{eV}$ and $1.788 \mathrm{eV}$, respectively. Those bands were assigned to the intra-shell luminescence of the $\mathrm{Co}^{2+}$ and $\mathrm{Fe}^{3+}$ ion. Both show several luminescence lines at lower energies, which were assigned to the phonon side band. Temperature dependent luminescence measurements show a decrease of the intra-shell luminescence with increasing measurement temperature. At $100 \mathrm{~K}$ only a broad luminescence was observed, which was assigned to the phonon side band, but the direct intra-shell luminescence was not obsorved for temperatures above $100 \mathrm{~K}$. At room temperature no intra-shell luminescence was observed. However, the acceptor, which is necessary for the excitation of the $\mathrm{Fe}^{3+}$ ion, is up to now unknown, but its temperature behavior plays a major role for room temperature intra-shell luminescence. The excitation process of the $\mathrm{Co}^{2+}$ ion is up to now unclear.

Power dependent photoluminescence measurements reveal an almost linear behavior, except for the excitation power densities above $\sim 300 \mathrm{~mW} / \mathrm{cm}^{2}$. Above this point the integrated intensity veers away from the linear behavior and shows an stronger intensity increase with increasing excitation power density. However, the assignment of this behavior to a changeover from spontaneous emission to stimulated emission is up to now not possible and needs further photoluminescence measurements, especially a decrease of the decay time could prove this situation.

All luminescence experiments on transition metal implanted $\mathrm{ZnO}$ nanowires, thin films as well as on single crystals had not shown any dimension effects [235]. Only the surface exciton emission in as-grown $\mathrm{ZnO}$ nanowire samples reveals some size effects. 


\section{Chapter 7}

\section{Summary and Outlook}

\subsection{Structural impact of ion implantation onto single crystals}

Within this thesis it was shown that the ion implantation of transition metals generates a huge amount of defects and results in an increasing Raman intensity of the $A_{1}(L O)$ phonon band. The intensity of this phonon band scales with the ion implantation fluence and expresses the ion implantation induced disorder. Annealing of argon implanted $\mathrm{ZnO}$ single crystals result in a healing of ion implantation induced defects and the Raman spectra after annealing had shown no difference to the initial state of pure $\mathrm{ZnO}$ for low dose Ar implanted ZnO. However, during annealing of transition metal implanted $\mathrm{ZnO}$ single crystals the $A_{1}(L O)$ disorder band decreases, whereby after annealing at $700{ }^{\circ} \mathrm{C}$ the intensity of this $A_{1}(L O)$ phonon band scales with the incorporated transition metal concentration. The annealing effect and the scaling with the transition metal concentration of the $A_{1}(L O)$ phonon band supports the assignment of interstitial zinc to this phonon mode $[316 ; 317]$. But further work is necessary to prove this assignment.

Only $\mathrm{Mn}$ implanted $\mathrm{ZnO}$ has shown an additional phonon mode in the Raman spectra, which scales with the Mn concentration and can be therefore assigned to a local vibration mode of $\mathrm{Mn}$ on a zinc lattice side in $\mathrm{ZnO}$.

No formation of secondary phases was observed in all transition metal implanted $\mathrm{ZnO}$ samples after annealing at $700{ }^{\circ} \mathrm{C}$ with transition metal concentrations below 8 at.\%. All TEM micrographs of transition metal implanted $\mathrm{ZnO}$ and Ar implanted $\mathrm{ZnO}$ reveal a high defect density after annealing at $700{ }^{\circ} \mathrm{C}$, which indicates a too low annealing temperature for a complete recovery. However, up to now the nature 
of ion implantation generated dislocation loops as well as their annealing behavior is unknow. Only studies of dislocation loop formation during electron irradiation were reported $[322 ; 327]$, revealing two different types of defects with different annealing temperatures. For a real understanding of the annealing of ion implantation generated dislocation loops a detailed knowledge of the nature is required and should be focused in future work.

\subsection{Secondary phases}

High dose transition metal implanted $\mathrm{ZnO}$ single crystals had shown additional features in Raman, XRD and TEM, which were assigned to secondary phase segregations in the $\mathrm{ZnO}$ matrix. It was shown that the results obtained by Raman spectroscopy and X-ray diffraction can differ due to their limitations to detect secondary phases. Therefore, a combination of several techniques for the detection of secondary phases is necessary, especially whenever the magnetic nature of transition metal alloyed $\mathrm{ZnO}$ samples is of interest. It was shown that small segregations have a major effect on the magnetic properties [14-18].

Annealing at different temperatures can result in the formation of different phases and/or a transformation into other oxide phases. Especially metallic segregations, which are observed after annealing at $700{ }^{\circ} \mathrm{C}$, were oxidized during the annealing at $900{ }^{\circ} \mathrm{C}$.

Some of the identified secondary phases are semiconductors, for example $\mathrm{NiO}$ and $\mathrm{CoO}$ with band-gaps of $4-4.3 \mathrm{eV}$ and $\sim 3$, respectively. Also the spinels $\mathrm{ZnMn}_{2} \mathrm{O}_{4}$, $\mathrm{ZnFe}_{2} \mathrm{O}_{4}$ and $\mathrm{ZnCo}_{2} \mathrm{O}_{4}$ are semicondutors with band gaps of $1.23 \mathrm{eV}, 1.92 \mathrm{eV}$, and $2.63 \mathrm{eV}[402 ; 406]$, respectively. Therefore, it is possible that the secondary phases have also an impact on the optical properties of high dose transition metal implanted $\mathrm{ZnO}$. However, an identification of those phases via luminescence studies requires a detailed knowledge of the luminescence properties of thos segregations. Up to now not much of the optical properties of the secondary phases is know.

\subsection{Defect luminescence}

With the radioactive ${ }^{111} \mathrm{In}$ isotope the assignment of the $\mathrm{I}_{9}$ luminescence line to shallow indium donors was approved and a defect band centered at about $2.85 \mathrm{eV}$ was found, which is induced by the daughter product of ${ }^{111} \mathrm{In}$ isotope: ${ }^{111} \mathrm{Cd}$. This broad band might be induced due to lattice deformation or differences in electronegativity. 
Two deep and broad luminescence bands were generated by the ion implantation of transition metals and/or argon into $\mathrm{ZnO}$, which are centered at about $1.9 \mathrm{eV}$ and $2.4 \mathrm{eV}$. The broad luminescence band at $1.9 \mathrm{eV}$ was assigned to interstitial oxygen with tetrahedral surroundings, which is introduced due to the competing process: recombination of interstitials and vacancies and the oxidation of ion implantation generated defects. It was shown that the assignment of the structured green luminescence band to copper acceptors is not certain. The green structured luminescence band was assigned to a transition from two different shallow donors to the same deep acceptor. The two shallow donors were identified as interstitial zinc with tetrahedral and octahedral surrounding, respectively. The deep acceptor level involved in this luminescence band was identified as interstitial oxygen with octahedral surrounding.

It was shown that the combination of these two deep luminescence bands results in an almost whitish luminescence emission, giving the opportunity for white light emitting diodes on basis of the deep defect center luminescence $\mathrm{ZnO}$. The next step would be the fabrication of an white LED on basis of this knowledge, to prove the efficiency of such a LED.

\subsection{Intra-shell luminescence}

For Co and Fe implanted $\mathrm{ZnO}$ samples sharp luminescence transitions at $1.878 \mathrm{eV}$ and $1.788 \mathrm{eV}$ were observed, which were assigned to the intra-shell luminescence of the $\mathrm{Co}^{2+}$ and $\mathrm{Fe}^{3+}$ ion, respectively. By temperature dependent luminescence measurements it was shown that the intensity of the intra-shell luminescence decreases with increasing measurement temperature and vanishes at room temperature. By power dependent photoluminescence measurements reveal an almost linear behavior for the excitation power densities, but above $\sim 300 \mathrm{~mW} / \mathrm{cm}^{2}$ the intensity of the intra-shell luminescence increases over-linear. However, the assignment of this behavior to a changeover from spontaneous emission to stimulated emission was up to now not possible and needs further photoluminescence measurements, especially decay time measurements.

Most reports dealing with intra-shell transitions of transition metal ions in $\mathrm{ZnO}$ were published in the sixties and seventies of the twentieth century $[21 ; 22 ; 368]$. Only little is known of the excitation mechanism of the intra-shell luminescence and should be treated in future work. 


\subsection{Transition metal implanted $\mathrm{ZnO}$ nanowires}

The incorporation of transition metals into $\mathrm{ZnO}$ nanowires was successful. By energy electron loss spectroscopy and energy dispersive X-ray spectroscopy the incorporation of transition metals up to a concentration of 4 at.\% was confirmed and no out-diffusion of the implanted transition metals was observed after annealing at $700{ }^{\circ} \mathrm{C}$. By photoluminescence measurements the successful activation of the transition metal ions in the $\mathrm{ZnO}$ host matrix was confirmed for the $\mathrm{Co}^{2+}$ and $\mathrm{Fe}^{3+}$ ions. Therefore, the ion implantation of transition metals is a promising technique for the incorporation of transition metals into $\mathrm{ZnO}$ nanowires. However, up to now, no difference between the optical porperties of transition metal implanted $\mathrm{ZnO}$ nanowires and $\mathrm{ZnO}$ single crystals was observed, but decay time measurements of the intra-shell luminescence could show a confinement due to the lower dimension of the nanowires [235]. 


\section{List of Figures}

2.1 Computed Curie temperatures by the mean field Zener model . . . . . . . . 17

2.2 Computed stabillity of the ferromagnetic state in $\mathrm{TM}$ alloyed $\mathrm{ZnO}$. . . . . 19

2.3 Representation of an bound magnetic polaron . . . . . . . . . . . . . 20

2.4 Crystal field subgroups of $\mathrm{O} h \ldots \ldots \ldots \ldots \ldots \ldots$

2.5 Schema of Jahn-Teller degeneration. . . . . . . . . . . . . . . 28

2.6 Band gap of $\mathrm{Zn}_{1-x} \mathrm{Mn}_{x} \mathrm{Se}$ as function of the Mn concentration $x \quad \ldots \ldots$

3.1 Schema of a hydrothermal growth system. . . . . . . . . . . . . . . . . 34

3.2 Schema of pulsed laser deposition of thin films. . . . . . . . . . . . . 37

3.3 Schematic drawing of the vapor-liquid-solid growth process is shown in combination with a simplified phase diagram (from [227]). . . . . . . . . . 40

3.4 HR-TEM micrograph of the interface between $\mathrm{ZnO}$ nanowire and $\mathrm{Yu}$ catalyst. 41

3.5 Tentative ternary Au-Zn-O phase diagram and tentative liquidus surface of the proposed phase diagram . . . . . . . . . . . . . . . . . . 42

3.6 Binary phase diagram of the system $\mathrm{Au}-\mathrm{Zn} \ldots \ldots \ldots$. . . . . . . . 43

3.7 Tentative isothermal section at $1100{ }^{\circ} \mathrm{C}$ of the proposed phase diagram. . . 44

3.8 Phase diagram of the $\mathrm{Mn}-\mathrm{O}$ and Fe-O systems . . . . . . . . . . . . 45

3.9 High temperature furnace for $\mathrm{ZnO}$ nanowire growth . . . . . . . . . . . 46

3.10 As grown $\mathrm{ZnO}$ nanowires and ion implantation profile for $\mathrm{Fe}$ in $\mathrm{ZnO}$. . . 48

4.1 TEM specimen produced by focused ion beam milling . . . . . . . . . 53

4.2 Schematic Raman spectrum . . . . . . . . . . . . . . . 56

4.3 Schematic drawing of a micro-Raman setup . . . . . . . . . . . . 57

4.4 Schematic drawing of the photoluminescence (PL) and cathodoluminescence $(\mathrm{CL})$ equipment. . . . . . . . . . . . . . . . . . 59

4.5 CIE primaries and chromaticity diagram . . . . . . . . . . . 61

5.1 Wurtzite lattice structure of $\mathrm{ZnO}$, the black and white colored spheres represent zinc and oxygen atoms, respectively. (from [291]) . . . . . . . . 64

5.2 $\mathrm{XRD}$-diffraction pattern of an as-grown $\mathrm{ZnO}$ nanowire sample and an virgin $\mathrm{ZnO}$ single crystals. . . . . . . . . . . . . . . . 6 65

5.3 TEM micrograph of an as-grown $\mathrm{ZnO}$ nanowire . . . . . . . . . . . 66

5.4 Raman spectra of pure $\mathrm{ZnO}$ recorded in different scattering configurations. $\quad 67$

5.5 Effect of ion implantation onto the phonon modes of $\mathrm{ZnO}$. . . . . . . . 70 
5.6 Raman spectra as function of annealing temperature for Ar and TM implanted $\mathrm{ZnO}$ single crystals . . . . . . . . . . . . . . . . 73

5.7 TEM micrograph of a 4 at.\% $\mathrm{Mn}$ implanted $\mathrm{ZnO}$ single crystal after annealing at $700{ }^{\circ} \mathrm{C} \ldots \ldots \ldots \ldots \ldots \ldots \ldots$

5.8 High resolution TEM micrograph of a 4 at.\% Co implanted ZnO nanowire . 76

5.9 Composition TM implanted ZnO nanowires recorded with EDS (a) and EELS. 77

5.10 Raman spectra of pure, $\mathrm{Mn}$ and Co implanted $\mathrm{ZnO}$ single crystals \& local vibration mode of $\mathrm{Mn}$ in $\mathrm{ZnO} \ldots \ldots \ldots$. . . . . . . . . . 78

5.11 Resonance measurements and detail view of the broad band between $500 \mathrm{~cm}^{-1}$ and $600 \mathrm{~cm}^{-1}$ in ZnO:Mn Raman spectra . . . . . . . . . . 79

5.12 Annealing behavior of the Raman modes at $519 \mathrm{~cm}^{-1}$ and $527 \mathrm{~cm}^{-1} \ldots$. . 80

$5.13 \mathrm{XRD}$ measurements of the $\mathrm{Ni}$ implanted $\mathrm{ZnO}$ samples after annealing at

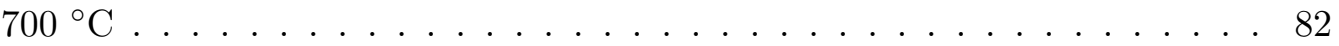

5.14 Low resolution TEM micrograph with EDS line scan along the cross section of a 16 at.\% $\mathrm{Mn}$ implanted $\mathrm{ZnO}$ single crystal after annealing at $700{ }^{\circ} \mathrm{C}$. . 83

5.15 The lateral inhomogeneity of the 16 at.\% Mn implanted $\mathrm{ZnO}$ single crystal due to precipitate formation, demonstrated by micro-Raman spectra taken after $700{ }^{\circ} \mathrm{C}$ annealing. . . . . . . . . . . . . . . . . . 84

$5.16 \mathrm{XRD}$ diffractogram of the 16 at.\% $\mathrm{Mn}$ implanted $\mathrm{ZnO}$ single crystal after $700{ }^{\circ} \mathrm{C}$ and $900{ }^{\circ} \mathrm{C}$ annealing. . . . . . . . . . . . . . . 86

5.17 High resolution TEM micrograph of the 16 at.\% Mn implanted $\mathrm{ZnO}$ single crystal annealing at $900{ }^{\circ} \mathrm{C} \ldots \ldots \ldots$. . . . . . . . . . . 88

5.18 Micro-Raman spectra of different spots on the 16 at.\% Co implanted $\mathrm{ZnO}$ after $700{ }^{\circ} \mathrm{C}$ annealing. . . . . . . . . . . . . . . . . 9 90

$5.19 \mathrm{XRD}$ diffractogram of the 16 at.\% Co implanted $\mathrm{ZnO}$ after annealing at

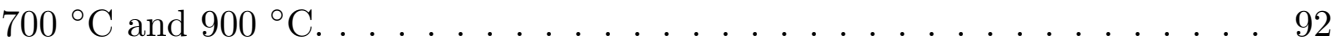

5.20 High resolution TEM micrograph of one large Co rich cluster in the 16 at.\% Co implanted $\mathrm{ZnO}$ single crystal after annealing at $900{ }^{\circ} \mathrm{C} \ldots \ldots$. . . . . . 93

5.21 Raman spectra of different spots on 16 at.\% Fe implanted $\mathrm{ZnO}$ single crystal after $700{ }^{\circ} \mathrm{C}$ annealing. . . . . . . . . . . . . . . . . . 95

5.22 XRD diffractogram of 16 at.\% Fe implanted $\mathrm{ZnO}$ single crystal after an-

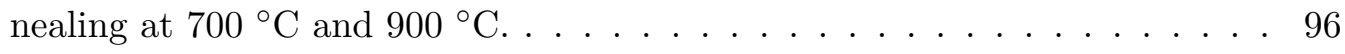

5.23 High resolution TEM micrograph of the 16 at.\% Fe implanted $\mathrm{ZnO}$ single crystal after annealing at $900{ }^{\circ} \mathrm{C} \ldots \ldots \ldots . \ldots \ldots 7$

$5.24 \mathrm{XRD}$ diffractogram of 16 at.\% $\mathrm{Ni}$ implanted $\mathrm{ZnO}$ single crystal after annealing at $700{ }^{\circ} \mathrm{C}$ and $900{ }^{\circ} \mathrm{C} \ldots \ldots \ldots \ldots 9 . \ldots \ldots$

5.25 High resolution TEM micrograph of the 16 at.\% Ni implanted $\mathrm{ZnO}$ single crystal after annealing at $900{ }^{\circ} \mathrm{C} \ldots \ldots \ldots \ldots$

6.1 Survey of $\mathrm{ZnO}$ luminescence at low temperature . . . . . . . . . 106

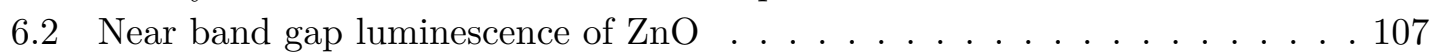

6.3 Photoluminescence of ${ }^{111} \mathrm{In}$ implanted $\mathrm{ZnO}$ as function of time. . . . . . . . 110

6.4 Survey of $\mathrm{ZnO}$ luminescence after transition metal implantation . . . . . . . 112

6.5 Schematic level arrangement of the oxygen vacancy intra-defect recombination and scheme of the configuration coordinate model . . . . . . . . . . 115 
6.6 DLTS measurement on the 5 at.\% V implanted ZnO PLD film. . . . . . . . 116

6.7 Photoluminescence effect of Ar/V implantation and annealing in air/oxygen ambient. . . . . . . . . . . . . . . . . . . 117

6.8 Temperature dependent photoluminescence of Ar implantation ZnO PLD thin film. . . . . . . . . . . . . . . . . . 118

6.9 Detail view of the structured green luminescence band. . . . . . . . . . . . 119

6.10 Temperature dependent $\mathrm{PL}$ of $\mathrm{V}$-implanted $\mathrm{ZnO}$. . . . . . . . . . . . . 122

6.11 Power dependent Potholuminescence of $\mathrm{V}$-implanted $\mathrm{ZnO}$. . . . . . . . . 123

6.12 Configuration of the $\mathrm{Co}^{2+}$ ion in $T_{d}$ symmetry (adapted from $[21 ; 22 ; 368$;

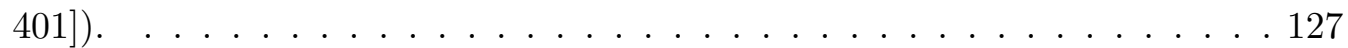

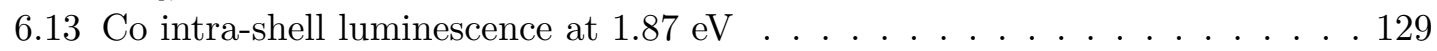

6.14 Photoluminescence of 8 at.\% Co implanted $\mathrm{ZnO}$ single crystal . . . . . . . . 131

6.15 Term scheme of $\mathrm{Fe}^{3+}$ ion in trigonal crystal fields. . . . . . . . . . . . . 133

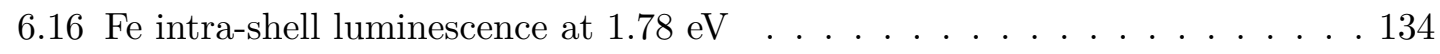

6.17 Temperature dependent photoluminescence of the $\mathrm{Fe}^{3+}$ intra-shell band . . 136

$6.18 \mathrm{Fe}^{3+}$ intra-shell luminescence at $1.78 \mathrm{eV}$ as function of excitation power . . 138

6.19 High power excitation of $\mathrm{Fe}^{3+}$ intra-shell luminescence . . . . . . . . . . . . 139

6.20 Luminescence of $\mathrm{Ni}$ implanted $\mathrm{ZnO}$ nanowires . . . . . . . . . . . . . . . . . 140

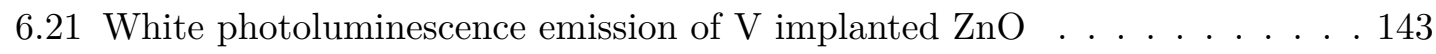

6.22 CIE of V implanted ZnO . . . . . . . . . . . . . . . . . . . . . . . . 144

6.23 Variance of the near band gap luminescence of $\mathrm{Mn}$ implanted $\mathrm{ZnO}$ single crystals and shift of peak position . . . . . . . . . . . . . 145 


\section{List of Tables}

2.1 Electronic configuration of the 3d-shell of transition metal ions in crystal fields [85]. At this point further perturbations by the crystal field are neglected and discussed in section 2.2. Therefore, the last row specifies the ground state term of the corresponding free ion. . . . . . . . . . . . . 14

2.2 Expected oxidation and charge state of some transition metals in II-VI and III-V semiconductors, whereas all $\mathrm{TM}^{2+}$ ions in $\mathrm{ZnO}$ are typical neutral $\left(\right.$ denoted as $\left.\mathrm{A}^{0} / \mathrm{D}^{0}\right)[89] \ldots \ldots \ldots \ldots \ldots \ldots$

2.3 Listed are the algebraic expressions of the tensor operators $C_{q}^{(k)}$ with $q \geq 0$ (after [113]). For negative $q: r_{+}=x+\mathrm{i} y$ is replaced with $r_{-}=x-\mathrm{i} y$ and the overall expression is multiplied by the factor $(-1)^{q}$. . . . . . . . 24

2.4 Non-vanishing crystal field parameters which are indicated by ' + ' for $+q$ and \pm if $+q$ and $-q$ parameters are non-zero (after [113]). . . . . . 26

2.5 Band gap $E_{g}$ of some 3 d-metal monoxides [130-132] . . . . . . . . . . . . 31

3.1 Energies, fluences and the corresponding transition metal concentration used within this thesis. For * marked series PLD thin films are used, which are annealed in oxygen ambient at $800^{\circ} \mathrm{C}$ for 30 minutes. For the Ar reference samples of this series we chose the same ion energy and ion fluence as for the 5 at.\% V implanted sample. . . . . . . . . . . . . . . . 48

5.1 Phonon modes in $\mathrm{ZnO}$ including their energy, Brillouin zone point and scattering symmetry in Raman (adapted from [88; 303-305]). . . . . . . . . 68

5.2 Magnetic Properties of potential or identified second phases (discussion for nanoparticels see text) . . . . . . . . . . . . . . . . . 102

6.1 List of free exciton (FX) and donor bound exciton (DX) lines and their chemical nature $\left({ }^{*}\right.$ indicate ionized donor bound exciton transitions $\left(\mathrm{D}^{-} \mathrm{X}\right)$, data and chemical assignment are taken from $[73-75 ; 107 ; 352]) . \ldots 108$

6.2 Collection of energy levels of $\mathrm{Co}^{2+}$ ( $d^{7}$ configuration) $3 d$-shell in $\mathrm{ZnO}$, which could contribute to the visible intra-shell luminescence including the ${ }^{4} \mathrm{~A}_{2}(\mathrm{~F})$

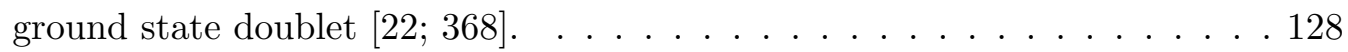

6.3 Phonon side band of the $\mathrm{Co}^{2+}$ intra-shell luminescence, the transition from the ${ }^{2} E(G)$ doublet to the ${ }^{4} A_{2}(F)$ ground state doublet is denoted with ZPL (zero phonon line). $\Delta E$ is the energy difference to the zero phonon line. The phonon modes are identified with respect to the references $[88 ; 303]$. . . 130 
6.4 Phonon side bands of the $\mathrm{Fe}^{3+}:{ }^{4} \mathrm{~T}_{1}(\mathrm{G}){ }^{6} \mathrm{~A}_{1}(\mathrm{~S})$ transition: spectral position $E$, difference to the zero phonon line (ZPL) $\Delta E$ and possible phonon modes

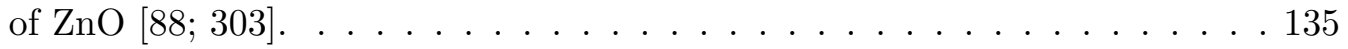




\section{Bibliography}

[1] O. Madelung et al. (editors): Landolf-Börnstein - Numerical Data and Functional Relationships in Science and Technology, volume 41b: Semiconductors of III. Springer (2002).

[2] S. Pearton et al.: Journal of Vacuum Science and Technology B 22(3), 932 (2004).

[3] T. Dietl et al.: Science 287, 1019 (2000).

[4] K. Sato et al.: Japanese Journal of Applied Physics Part 2-Letters 39(6B), L555 (2000).

[5] K. Sato et al.: Japanese Journal of Applied Physics Part 2-Letters 40(4A), L334 (2001).

[6] S. J. Pearton et al.: Journal of Electronic Materials 36(4), 462 (2007).

[7] C. Zener: Physical Review 82(3), 403 (1951).

[8] F. Pan et al.: Materials Science ES Engineering R-Reports 62(1), 1 (2008).

[9] J. M. D. Coey et al.: Nature Materials 4(2), 173 (2005).

[10] M. Herbich et al.: Physical Review B 58(11), 7024 (1998).

[11] C. Liu et al.: Journal of Materials Science: Materials in Electronics 16(9), 555 (2005).

[12] S. Pearton et al.: Semiconductor Science and Technology 19(10), R59 (2004).

[13] S. J. Pearton et al.: Journal of Applied Physics 93(1), 1 (2003).

[14] K. Potzger et al.: Journal of Applied Physics 104(2), 023510 (2008).

[15] S. Q. Zhou et al.: Physical Review B 77(3), 035209 (2008).

[16] S. Q. Zhou et al.: Journal of Physics D 40(4), 964 (2007).

[17] S. Q. Zhou et al.: Journal of Applied Physics 103(2), 023902 (2008).

[18] S. Q. Zhou et al.: Journal of Applied Physics 100(11), 114304 (2006).

[19] M. Schumm et al.: Journal of Applied Physics submitted (2009).

[20] X. Wang et al.: Applied Physics Letters 91(3), 031908 (2007).

[21] R. Anderson: Physical Review 164(2), 398 (1967).

[22] H. Weakliem: Journal of Chemical Physics 36(8), 2117 (1962).

[23] E. Malguth et al.: Physica Status Solidi B 245(3), 455 (2008).

[24] R. Heitz et al.: Physical Review B 45(16), 8977 (1992).

[25] R. Heitz et al.: Journal of Luminescence 48-49(pt 2), 689 (1991). 
[26] A. Polman: Journal of Applied Physics 82(1), 1 (1997).

[27] IBM Zurich Research Laboratory: Optical interconnects (2009). URL http://www.zurich.ibm.com/st/ server/interconnects.html. [Online; accessed 25-February-2009].

[28] D. A. B. Miller: International Journal of Optoelectronics 11(3), 155 (1997).

[29] D. A. B. Miller: Proceedings of The IEEE 88(6), 728 (2000).

[30] Y. Huang et al.: Science 294, 1313 (2001).

[31] Y. Huang et al.: Science 291, 630 (2001).

[32] Y. Huang et al.: Small 1(1), 142 (2005).

[33] J. Wang et al.: Science 293(5534), 1455 (2001).

[34] Y. Cui et al.: Science 293, 1289 (2001).

[35] C. Lieber: MRS Bulletin 28(7), 486 (2003).

[36] M. A. Zimmler et al.: Nano Letters 8(6), 1695 (2008).

[37] M. A. Zimmler et al.: Applied Physics Letters 93(5), 051101 (2008).

[38] T. Voss et al.: Nano Letters 7(12), 3675 (2007).

[39] Z. Wang (editor): Nanowires and Nanobelts - Materials, Properties and Devices, volume 1: Metal and Semiconductor Nanowires. Kluwer Academic Publishers, Boston (2004).

[40] C. Borchers et al.: Journal of Physical Chemistry B 110(4), 1656 (2006).

[41] R. Wagner et al.: Applied Physics Letters 4(5), 89 (1964).

[42] L. Liao et al.: Journal of Applied Physics 102(11), 114307 (2007).

[43] H. Pan et al.: Journal of Nanoscience and Nanotechnology 7(10), 3620 (2007).

[44] X. F. Wang et al.: Nanotechnology 19(45), 455702 (2008).

[45] B. Zhang et al.: Chinese Science Bulletin 53(11), 1639 (2008).

[46] W. B. Jian et al.: Journal of Nanoscience and Nanotechnology 8(1), 202 (2008).

[47] C. Ronning et al.: Applied Physics Letters 84(5), 783 (2004).

[48] Z. Y. Wu et al.: New Journal of Physics 10, 033017 (2008).

[49] S. Müller et al.: Journal of Applied Physics 104(12), 123504 (2008).

[50] S. Müller et al.: Nanotechnology 20(13), 135704 (2009).

[51] E. Schubert: Light-Emitting Diodes. Cambridge University Press, 2nd edition (2006).

[52] S. Nakamura: Solid State Communications 102(2-3), 237 (1997).

[53] S. Nakamura: Electronics and Communications in Japan Part II-Electronics 81(5), 1 (1998).

[54] E. Chase et al.: Journal of Applied Physics 40(6), 2512 (1969).

[55] H. Lozykowski et al.: Applied Physics Letters 74(8), 1129 (1999).

[56] W. Jadwisienczak et al.: Journal of Applied Physics 89(8), 4384 (2001). 
[57] T. Monteiro et al.: Journal of Applied Physics 93(11), 8995 (2003).

[58] U. Vetter et al.: Applied Physics Letters 84(21), 4286 (2004).

[59] J. Tsao (editor): Light Emitting Diodes (LEDs) for General Illumination. Optoelectronics Industry Development Association (2002). URL http://apps1.eere.energy.gov/buildings/publications/pdfs/ssl/ report_led_november_2002a_1.pdf.

[60] US Department of Energy (DOE) - Energy Efficiency and Renewable Energy: Solid state lightning - technology roadmaps. URL http://www1.eere.energy.gov/buildings/ssl/techroadmaps.html.

[61] Y. Narukawa et al.: Japanese Journal of Applied Physics Part 2 41(4A), L371 (2002).

[62] I. E. Titkov et al.: Semiconductors 41(5), 564 (2007).

[63] C. Klingshirn: Physica Status Solidi B 244(9), 3027 (2007).

[64] D. C. Reynolds et al.: Solid State Communications 101(9), 643 (1997).

[65] X. L. Wu et al.: Applied Physics Letters 78(16), 2285 (2001).

[66] X. Guo et al.: Japanese Journal of Applied Physics Part 2 40, L177 (2001).

[67] A. Tsukazaki et al.: Nature Materials 4, 42 (2005).

[68] K.-K. Kim et al.: Applied Physics Letters 83(1), 63 (2003).

[69] D. C. Look et al.: Physica Status Solidi B 241(3), 624 (2004).

[70] F. H. Leiter et al.: Physica Status Solidi B 226(1), R4 (2001).

[71] F. Leiter et al.: Physica B 340-342, 201 (2003).

[72] P. S. Xu et al.: Chinese Physics Letters 18(9), 1252 (2001).

[73] B. Meyer et al.: Physica Status Solidi B 241(2), 231 (2004).

[74] K. Johnston et al.: Physical Review B 73, 165212 (2006).

[75] S. Müller et al.: Applied Physics Letters 90(1), 012107 (2007).

[76] S. Wolf et al.: Science 294(5546), 1488 (2001).

[77] S. J. Pearton et al.: IEE Proceedings-Circuits Devices And Systems 152(4), 312 (2005).

[78] J. K. Furdyna: Journal of Applied Physics 64(4), R29 (1988).

[79] M. Jain (editor): Diluted Magnetic Semiconductors. World Scientific, Singapore (1991).

[80] H. Ohno: Science 291(5505), 840 (2001).

[81] T. Jungwirth et al.: Physical Review B 72(16), 165204 (2005).

[82] T. Jungwirth et al.: Reviews of Modern Physics 78(3), 809 (2006).

[83] E. Rita et al.: Applied Physics Letters 85(21), 4899 (2004).

[84] U. Wahl et al.: Physical Review B 69(1), 012102 (2004).

[85] E. Omel'yanovskii et al.: Transition Metal Impurities in Semiconductors. Adam Hilger LTD, Bristol (1986).

[86] D. Meschede (editor): Gerthsen Physik. Springer, Berlin, 21st edition (2002). 
[87] Wikipedia: List of hund's rules — wikipedia, the free encyclopedia (2008). URL http://en.wikipedia.org/ w/index.php?title=List_of_Hund\%27s_rules\&oldid=259976929. [Online; accessed 8-January-2009].

[88] H.-J. Schulz et al.: Physical Review B 35, 18 (1987).

[89] T. Graf et al.: Physica Status Solidi B 239(2), 277 (2003).

[90] R. Dingle: Physical Review Letters 23, 579 (1969).

[91] C. Kittel: Einführung in die Festkörperphysik. Oldenbourg Verlag, München, 13th edition (2002).

[92] M. Getzlaff: Fundamentals of Magnetism. Springer, Berlin (2008).

[93] R. Janisch et al.: Journal of Physics Condensed Matter 17(27), (2005).

[94] T. Dietl: Semiconductor Science And Technology 17(4), 377 (2002).

[95] C. Zener: Physical Review 81(3), 440 (1951).

[96] C. Zener: Physical Review 83(2), 299 (1951).

[97] S. Pearton et al.: Progress in Materials Science 50(3), 293 (2005).

[98] U. Oezguer et al.: Journal of Applied Physics 98(4), 041301 (2005).

[99] K. Sato et al.: Semiconductor Science and Technology 17(4), 367 (2002).

[100] K. Sato et al.: Physica Status Solidi B 229(2), 673 (2002).

[101] H. Katayama-Yoshida et al.: Physica Status Solidi A 204(1), 15 (2007).

[102] H. Katayama-Yoshida et al.: Applied Physics A 89(1), 19 (2007).

[103] T. Fukushima et al.: Japanese Journal of Applied Physics Part 2-Letters 43(11A), L1416 (2004).

[104] H. Katayama-Yoshida et al.: Journal of Magnetism and Magnetic Materials 310(2), 2070 (2007).

[105] K. Sato et al.: Physica B 376, 639 (2006).

[106] J. W. Orton et al.: Reports on Progress in Physics 61(1), 1 (1998).

[107] B. Meyer et al.: Semiconductor Science and Technology 20, S62 (2005).

[108] J. B. Torrance et al.: Physical Review Letters 29(17), 1168 (1972).

[109] A. C. Durst et al.: Physical Review B 65(23), 235205 (2002).

[110] P. A. Wolff et al.: Journal of Applied Physics 79(8), 5196 (1996).

[111] D. E. Angelescu et al.: Physical Review B 65(7), 075211 (2002).

[112] H. Bethe: Annalen der Physik 395(2), 133 (1929).

[113] D. Newman et al. (editors): Crystal Field Handbook. Cambridge University Press, Cambridge (2000).

[114] R. Schlapp: Physical Review 41(2), 194 (1932).

[115] W. G. Penney: Physical Review 42(5), 666 (1932).

[116] J. Van Vleck: Physical Review 41(2), 208 (1932).

[117] S. Gasiorowicz: Quantenphysik. Oldenbourg Verlag, München (2002).

[118] C. Morrison: Crystal Fields for Transition-Metal Ions in Laser Host Materials. Springer, Berlin (1994). 
[119] M. Gerloch et al.: Ligand-Field Parameters. Cambridge University Press, London (1973).

[120] J. Griffith: The Theory of Tranition-Metal ions. Cambridge University Press, London (1961).

[121] S. Sugano et al.: In: Multiplets of Tranistion-Metal Ions in Crystals, volume 33 of Pure and Applied Physics. Academic Press, New York (1970).

[122] D. S. McClure: Solid State Physics 9, 399 (1959).

[123] Wikipedia: Kramers theorem - wikipedia, the free encyclopedia (2008). URL http://en.wikipedia.org/ w/index.php?title=Kramers_theorem\&oldid=206111048. [Online; accessed 12-January-2009].

[124] Wikipedia: Jahn-teller effect - wikipedia, the free encyclopedia (2008). URL http://en.wikipedia.org/w/ index.php?title=Jahn\%E2\%80\%93Teller_effect\&oldid=247455580. [Online; accessed 13-January-2009].

[125] K. Stevens: Magnetic Ions in Crystals. Princeton University Press, Princeton (1998).

[126] A. Hoffmann et al.: Zeitschrift für Physikalische Chemie 201, 3 (1997).

[127] A. Hoffmann et al.: Journal of Crystal Growth 101(1-4), 385 (1990).

[128] U. G. Kaufmann et al.: Journal of Physics C 7(4), 791 (1974).

[129] T. Makino et al.: Applied Physics Letters 78(9), 1237 (2001).

[130] V. I. Anisimov et al.: Journal of Physics 2(17), 3973 (1990).

[131] C. DiazGuerra et al.: Solid State Communications 104(12), 763 (1997).

[132] T. Ito et al.: Journal of Materials Science 33(14), 3555 (1998).

[133] R. B. Bylsma et al.: Physical Review B 33(12), 8207 (1986).

[134] S. V. Bhat et al.: Solid State Communications 135(6), 345 (2005).

[135] K. Samanta et al.: Physical Review B 73(24), 245213 (2006).

[136] K. Samanta et al.: Applied Physics Letters 90(26), 261903 (2007).

[137] H. J. Zhou et al.: Physica Status Solidi A 204(1), 112 (2007).

[138] N. Wooster et al.: Nature 157(3984), 297 (1946).

[139] E. Ohshima et al.: Journal of Crystal Growth 260(1-2), 166 (2004).

[140] W. J. Li et al.: Journal of Crystal Growth 203(1-2), 186 (1999).

[141] T. Sekiguchi et al.: Journal of Crystal Growth 214-215, 72 (2000).

[142] L. N. Dem'yanets et al.: Journal of Crystal Growth 310(5), 993 (2008).

[143] R. A. Laudise: Chemical \& Engineering News 65(39), 30 (1987).

[144] R. A. Laudise et al.: Journal of Physical Chemistry 64(5), 688 (1960).

[145] R. A. Laudise et al.: Journal of The American Ceramic Society 47(1), 9 (1964).

[146] D. C. Reynolds et al.: Journal of Applied Physics 95(9), 4802 (2004).

[147] S.-J. Han et al.: Applied Physics Letters 81(22), 4212 (2002).

[148] S. Kolesnik et al.: Journal of Applied Physics 95(5), 2582 (2004).

[149] S. Kolesnik et al.: Journal of Superconductivity 15(4), 251 (2002). 
[150] P. Sharma et al.: Nature Materials 2(10), 673 (2003).

[151] Wikipedia: Hydrothermal synthesis - wikipedia, the free encyclopedia (2008). URL http://en.wikipedia. org/w/index.php?title=Hydrothermal_synthesis\&oldid=252567350. [Online; accessed 15-January-2009].

[152] J. Nause et al.: Us patent 5900060 - pressurized skull cruciable apparatus for crystal growth and related system and methods (1999). URL http://www.patentstorm.us/patents/5900060/fulltext.html.

[153] J. Nause et al.: Us patent 6936101 - semi-insulating bulk zinc oxide single crystal (2005). URL http: //www.patentstorm.us/patents/6936101/fulltext.html.

[154] M. H. Kane et al.: Journal of Applied Physics 97(2), 023906 (2005).

[155] M. H. Kane et al.: Physica Status Solidi B 244(5), 1462 (2007).

[156] M. Torii et al.: IEEE Transactions On Magnetics 16(5), 879 (1980).

[157] E. Guzman et al.: Annalen der Physik (Leipzig) 13(1-2), 57 (2004).

[158] T. Mizokawa et al.: Physical Review B 65(8), 085209 (2002).

[159] S. Ramachandran et al.: Applied Physics Letters 84(25), 5255 (2004).

[160] K. Rode et al.: Journal of Applied Physics 93(10), 7676 (2003).

[161] K. Ueda et al.: Applied Physics Letters 79(7), 988 (2001).

[162] T. Wakano et al.: Physica E 10(1-3), 260 (2001).

[163] Q. Y. Xu et al.: Applied Physics Letters 91(9), 092503 (2007).

[164] L. Yan et al.: Journal of Applied Physics 96(1), 508 (2004).

[165] Y. Zheng et al.: Journal of Crystal Growth 274(1-2), 156 (2005).

[166] J.-L. Zhao et al.: Journal of Crystal Growth 276, 507 (2005).

[167] M. Diaconu et al.: Solid State Communications 137(8), 417 (2006).

[168] Z. Jin et al.: Physica E 10(1-3), 256 (2001).

[169] J. H. Kim et al.: Journal of Applied Physics 92(10), 6066 (2002).

[170] A. Fouchet et al.: Journal of Applied Physics 95(11), 7187 (2004).

[171] W. Prellier et al.: Applied Physics Letters 82(20), 3490 (2003).

[172] E. Biegger et al.: Journal of Applied Physics 101(7), 073904 (2007).

[173] Y. M. Cho et al.: Applied Physics Letters 80(18), 3358 (2002).

[174] A. Dinia et al.: Journal of Applied Physics 97(12), 123908 (2005).

[175] S. Lee et al.: Material Science and Engineering B 126(2-3), 300 (2006).

[176] N. Theodoropoulou et al.: Journal of Magnetism and Magnetic Materials 300(2), 407 (2006).

[177] X. X. Wei et al.: Journal of Physics 18(31), 7471 (2006).

[178] H. Y. Xu et al.: Applied Physics Letters 88(24), 242502 (2006).

[179] Z. B. Gu et al.: Thin Solid Films 515(4), 2361 (2006).

[180] Y. M. Kim et al.: Solid State Communications 129(3), 175 (2004). 
[181] G. Lawes et al.: Physical Review B 71(4), 045201 (2005).

[182] J. H. Park et al.: Applied Physics Letters 84(8), 1338 (2004).

[183] A. S. Risbud et al.: Physical Review B 68(20), 205202 (2003).

[184] S. W. Yoon et al.: Journal of Applied Physics 93(10), 7879 (2003).

[185] G. Srinivasan et al.: Journal of Crystal Growth 310(7-9), 1841 (2008).

[186] A. Mofor et al.: Physica Status Solidi C 3, 1104 (2006).

[187] Wikipedia: Pulsed laser deposition — wikipedia, the free encyclopedia (2008). URL http://en.wikipedia. org/w/index .php?title=Pulsed_laser_deposition\&oldid=259950687. [Online; accessed 16-January-2009].

[188] D. Chrisey et al. (editors): Pulsed laser deposition of thin films. Wiley, New York (1994).

[189] H. M. Christen et al.: Journal of Physics 20(26), 264005 (2008).

[190] Z. W. Jin et al.: Applied Physics Letters 83(1), 39 (2003).

[191] S. W. Jung et al.: Applied Physics Letters 80(24), 4561 (2002).

[192] J. L. Maurice et al.: European Physical Journal 33(2), 109 (2006).

[193] Wikipedia: Sputtering — wikipedia, the free encyclopedia (2009). URL http://en.wikipedia.org/w/index. php?title=Sputtering\&oldid=262624096. [Online; accessed 16-January-2009].

[194] N. Croitoru et al.: Thin Solid Films 150(2-3), 291 (1987).

[195] B. X. Lin et al.: Applied Physics Letters 79(7), 943 (2001).

[196] Y. X. Jin et al.: Journal of Physics 13(44), L913 (2001).

[197] J. Ma et al.: Journal of Vacuum Science and Technology B 22(1), 94 (2004).

[198] Wikipedia: Sol-gel — wikipedia, the free encyclopedia (2009). URL http://en.wikipedia.org/w/index.php? title=Sol-gel\&oldid=262899125. [Online; accessed 16-January-2009].

[199] Y. Heo et al.: Materials Science and Engineering: R: Reports 47, 1 (2004).

[200] M. J. Xin et al.: Materials Letters 62(17-18), 2717 (2008).

[201] I. Djerdj et al.: Journal of Materials Chemistry 18(43), 5208 (2008).

[202] L. Schmidt-Mende et al.: Materials Today 10(5), 40 (2007).

[203] L. E. Greene et al.: Inorganic Chemistry 45(19), 7535 (2006).

[204] J. Park et al.: Journal of Physical Chemistry B 108(36), 13594 (2004).

[205] D. Zitoun et al.: Journal of The American Chemical Society 127(43), 15034 (2005).

[206] X. H. Zhong et al.: Journal Of Physical Chemistry B 110(1), 2 (2006).

[207] K. An et al.: Journal Of The American Chemical Society 128(30), JA0608702 (2006).

[208] C. K. Xu et al.: Chemical Physics Letters 366(5-6), 567 (2002).

[209] L. L. Wu et al.: Materials Letters 58(21), 2700 (2004).

[210] C. K. Xu et al.: Journal of Crystal Growth 255(3-4), 308 (2003).

[211] W. Z. Wang et al.: Chemical Physics Letters 362(1-2), 119 (2002). 
[212] S. Vaidya et al.: Journal of Physical Chemistry C 112(33), 12610 (2008).

[213] L. Y. Bai et al.: Materials Letters 61(8-9), 1698 (2007).

[214] Q. Yang et al.: Materials Letters 59(14-15), 1967 (2005).

[215] Y. Lin et al.: Chemical Physics Letters 380(5-6), 521 (2003).

[216] S. I. Kim et al.: Applied Physics Letters 93(3), 033503 (2008).

[217] G. X. Wang et al.: Journal of Physical Chemistry C 112(39), 15220 (2008).

[218] C. W. Na et al.: Applied Physics Letters 87(14), 142504 (2005).

[219] Y. J. Xiong et al.: Chemistry-A European Journal 9(7), 1645 (2003).

[220] X. Wang et al.: Journal of The American Chemical Society 124(12), 2880 (2002).

[221] X. Wang et al.: Chemistry-A European Journal 9(1), 300 (2003).

[222] D. S. Zheng et al.: Journal of Physical Chemistry B 109(34), 16439 (2005).

[223] K. M. Shaju et al.: Physical Chemistry Chemical Physics 9(15), 1837 (2007).

[224] W. R. Liu et al.: Journal of Applied Crystallography 40, 924 (2007).

[225] L. Q. Liu et al.: Applied Physics Letters 88(6), 063104 (2006).

[226] A. O. Ankiewicz et al.: Journal of Applied Physics 101(2), 024324 (2007).

[227] D. Stichtenoth: Wachstum und Modifikation von Halbleiternanodrähten. Master's thesis, Georg-AugustUniversität zu Göttingen (2005).

[228] A. Morales et al.: Science 279, 208 (1998).

[229] T. Martensson et al.: Nano Letters 4(10), 1987 (2004).

[230] N. Panev et al.: Applied Physics Letters 83(11), 2238 (2003).

[231] L. Samuelson et al.: Physica E 25(2-3 SPEC.ISS.), 313 (2004).

[232] E. Sutter et al.: Nano Letters 8(2), 411 (2008).

[233] X. Duan et al.: Advanced Materials 12(4), 298 (2000).

[234] C. Palacios et al.: Geologische Rundschau 86(1), 132 (1997).

[235] D. Stichtenoth: Dimensionseffekte in Halbleiternanodrähten. Ph.D. thesis, Georg-August-Universität zu Göttingen (2008).

[236] S. Müller et al.: In: DPG spring meeting 2005. Berlin (2005).

[237] S. Müller: Wachstum von ZnO Nanodrähtenhten und deren Dotierung durch Ionenstrahlen. Master's thesis, Georg-August-Universität Göttingen (2005).

[238] A. V. Makarov et al.: Inorganic Materials 39(6), 594 (2003).

[239] H. Okamoto: Journal of Phase Equilibria and Diffusion 27(4), 427 (2006).

[240] X. D. Wang et al.: Journal of Physical Chemistry B 108(26), 8773 (2004).

[241] H. Hanemann et al.: In: Ternäre Legierungen des Aluminiums, volume III of Atlas Metallographicus. Verlag Stahleisen, Düsseldorf, Germany (1952). 
[242] B. Predel: In: Li-Mg - Nd-Zr, volume 5H of Landolt-Börnstein - Group IV Physical Chemistry, chapter Mn-O (Manganese-Oxygen), 1-2. Springer (1997).

[243] B. Predel: In: Dy-Er - Fr-Mo, volume 5e of Landolt-Börnstein - Group IV Physical Chemistry, chapter Fe-O (Iron-Oxygen), 1-5. Springer (1995).

[244] P. Franke et al.: In: Binary systems. Part 4: Binary Systems from Mn-Mo to Y-Zr, volume 19B4 of LandoltBörnstein - Group IV Physical Chemistry, chapter Fe-O, 1-2. Springer (2006).

[245] P. Franke et al.: In: Binary systems. Part 3: Binary Systems from Cs-K to Mg-Zr, volume 19B3 of LandoltBörnstein - Group IV Physical Chemistry, chapter Fe-O, 1-3. Springer (2005).

[246] O. Madelung et al.: In: Non-Tetrahedrally Bonded Binary Compounds II, volume 41D of Landolt-Börnstein Group III Condensed Matter, chapter CoO: phase diagram, crystal structure, lattice parameters, 1-5. Springer (1993).

[247] B. Predel: In: Ni-Np - Pt-Zr, volume 5I of Landolt-Börnstein - Group IV Physical Chemistry, chapter Ni-O (Nickel-Oxygen), 1-1. Springer (1998).

[248] B. Predel: In: $C a-C d-C o-Z r$, volume 5c of Landolt-Börnstein - Group IV Physical Chemistry, chapter Co-O (Cobalt-Oxygen), 1-4. Springer (1993).

[249] B. Predel: In: Ac-Au - Au-Zr, volume 5a of Landolt-Börnstein - Group IV Physical Chemistry, chapter Au-Fe (Gold-Iron), 1-5. Springer (1991).

[250] B. Predel: In: Ac-Au - Au-Zr, volume 5a of Landolt-Börnstein - Group IV Physical Chemistry, chapter Au-Ni (Gold-Iron), 1-6. Springer (1991).

[251] B. Predel: In: Ac-Au - Au-Zr, volume 5a of Landolt-Börnstein - Group IV Physical Chemistry, chapter Au-Mn (Gold-Manganese), 1-5. Springer (1991).

[252] B. Predel: In: Ac-Au - Au-Zr, volume 5a of Landolt-Börnstein - Group IV Physical Chemistry, chapter Au-Co (Gold-Cobalt), 1-4. Springer (1991).

[253] Y. Heo et al.: Applied Physics Letters 84(13), 2292 (2004).

[254] D. Norton et al.: Applied Physics Letters 83(26), 5488 (2003).

[255] N. A. Theodoropoulou et al.: Solid State Electronics 47(12), 2231 (2003).

[256] A. F. Vyatkin et al.: Nuclear Instruments 83 Methods in Physics Research Section B 237(1-2), 179 (2005).

[257] P. Wu et al.: Applied Physics Letters 89(1), 012508 (2006).

[258] I. J. Chen et al.: Journal of Physical Chemistry C 112(25), 9168 (2008).

[259] W. B. Jian et al.: Physical Review B 73(23), 233308 (2006).

[260] L. Liao et al.: Nanotechnology 17, 830 (2006).

[261] Z. Y. Wu et al.: Nanotechnology 17(21), 5511 (2006).

[262] K. Ip et al.: Journal of Vacuum Science \& Technology B 21(4), 1476 (2003).

[263] J. Mayer et al.: Ion Implantation in Semiconductors. Academic Press (1970).

[264] R. Wilson et al.: Ion Beams. Wiley Interscience Publication (1973).

[265] J. Ziegler et al.: The Stopping and Range of Ions in Solids. Pergamon Press, New York (1999). URL http://www.srim.org/.

[266] M. Uhrmacher et al.: Nuclear Instruments 83 Methods In Physics Research Section B 240(1-2), 48 (2005). 
[267] Carl Zeiss SMT Ltd.: Handbuch für die Rasterelektronenmikroskope Supra(VP) und Ultra. Carl Zeiss SMT Ltd. (2004).

[268] T. Everhart et al.: Journal of Scientific Instruments 37(7), 246 (1960).

[269] L. A. Giannuzzi et al.: Micron 30(3), 197 (1999).

[270] P. J. Heaney et al.: American Mineralogist 86(9), 1094 (2001).

[271] L. Reimer: Springer, Berlin (1998).

[272] L. Pages et al.: Atomic Data 4, 1 (1972).

[273] B. Cullity et al.: Elements of X-Ray Diffraction. Prentice Hall, 3rd edition (2001).

[274] B. Warren: X-Ray diffraction. Reprint. Dover Publications, New York (1990). Originally publ. by AddisonWesley, Reading, Mass., 1969.

[275] International Centre for Diffraction Data: Pcpdfwin 1.30 (1997).

[276] P. Scherrer: Nachrichten von der Gesellschaft der Wissenschaften zu Göttingen 26, 98 (1918).

[277] Bruker Analytical X-Ray Systems: D8 Advance, D8 Discover - X-ray Diffractometer, ii edition (1999).

[278] P. Brüesch: In: M. Cardona et al. (editors) Phonons: Theory and Experiments II, volume 65 of Springer Series in Solid-State Sciences. Springer, Berlin (1986).

[279] In: M. Cardona et al. (editors) Light Scattering in Solids VIII, volume 76 of Topics in Applied Physics. Springer, Berlin (2000).

[280] C. Hamaguchi: Basic Semiconductor Physics. Springer, Berlin (2001).

[281] M. Schumm: ZnO-based semiconductors studied by Raman spectroscopy: semimagnetic alloying, doping and nanostructures. Ph.D. thesis, Julius-Maximilians-Universität Würzburg (2008).

[282] C. Sansonetti et al.: Applied Optics 35(1), 74 (1996).

[283] D. MacAdam: In: Color Measurement, volume 27 of Springer Series in Optical Sciences, 4-25. Springer, Berlin Heidelberg, 2nd edition (1985).

[284] B. Fortner et al.: Number by colors. Springer-Verlag, New York (1997).

[285] W. Wright: The measurement of colour. Adam Hilger LTD, London, 4th edition (1969).

[286] M. Grundmann: The Physics of Semiconductors. Springer, Berlin Heidelberg (2006).

[287] D. V. Lang: Journal of Applied Physics 45(7), 3023 (1974).

[288] D. V. Lang: Journal of Applied Physics 45(7), 3014 (1974).

[289] H. von Wenckstern et al.: Applied Physics Letters 84(1), 79 (2004).

[290] J. Ziegler (editor): Ion Implantation Technology. North-Holland, Amsterdam (1992).

[291] J. Jaffe et al.: Physical Review B 48, 7903 (1993).

[292] A. B. M. A. Ashrafi et al.: Applied Physics Letters 76(5), 550 (2000).

[293] A. A. Ashrafi et al.: Journal of Crystal Growth 221, 435 (2000).

[294] EMS Online: Electron microscopy image simulation. http://cimesg1.epfl.ch/CIOLS/crystal1.pl (2005). URL http://cecm.insa-lyon.fr/CIOLS/crystal1.pl.

[295] X. Wang et al.: Nano Letters 4(3), 423 (2004). 
[296] D. Banerjee et al.: Applied Physics A 80, 749 (2005).

[297] H. Fan et al.: Nanotechnology 16, 913 (2005).

[298] J. Song et al.: Journal of Physical Chemistry B 109, 9869 (2005).

[299] J.-S. Lee et al.: Journal of Crystal Growth 254, 423 (2003).

[300] Z. Wang: Journal of Physics 16, R829 (2004).

[301] T. Damen et al.: Physical Review 142(2), 570 (1966).

[302] R. Loudon: Advances In Physics 13(52), 423 (1964).

[303] R. Cusco et al.: Physical Review B 75(16), 165202 (2007).

[304] W. Wegener et al.: Physics Letters A 31(1), 2 (1970).

[305] B. H. Bairamov et al.: Physica Status Solidi B 119(1), 227 (1983).

[306] Z. Jin et al.: Journal of Crystal Growth 214/215, 55 (2000).

[307] C. H. Bates et al.: Journal of Inorganic \& Nuclear Chemistry 28(2), 397 (1966).

[308] F. W. Kleinlein et al.: Zeitschrift für Physik A Hadrons and Nuclei 266(3), 201 (1974).

[309] Z. Q. Chen et al.: Journal of Applied Physics 99(9), 093507 (2006).

[310] Z. Q. Chen et al.: Journal of Applied Physics 97(1), 013528 (2005)

[311] T. S. Jeong et al.: Journal of Applied Physics 96(1), 175 (2004).

[312] S. Venkataraj et al.: Journal of Applied Physics 102(1), 014905 (2007).

[313] F. Friedrich et al.: Applied Physics Letters 91(11), 111903 (2007).

[314] J. M. Liu et al.: Ferroelectrics 231(1), 223 (1999).

[315] G. J. Exarhos et al.: Thin Solid Films 270(1-2), 27 (1995).

[316] C. J. Youn et al.: Journal of Crystal Growth 261(4), 526 (2004).

[317] M. Tzolov et al.: Thin Solid Films 379(1-2), 28 (2000).

[318] S. O. Kucheyev et al.: Physical Review B 67(9), 094115 (2003).

[319] S. O. Kucheyev et al.: Vacuum 73(1), 93 (2004).

[320] S. O. Kucheyev et al.: Physical Review B 64(3), 035202 (2001).

[321] S. O. Kucheyev et al.: Materials Science and Engineering: R: Reports 33(2-3), 51 (2001).

[322] T. Yoshiie et al.: Philosophical Magazine A 41(6), 935 (1980).

[323] K. Vanheusden et al.: Applied Physcis Letters 68(3), 403 (1996).

[324] J. S. Thakur et al.: Journal of Applied Physics 102(9), 093904 (2007).

[325] M. Schumm et al.: New Journal of Physics 10, 043004 (2008).

[326] C. Borchers et al.: Nanotechnology 17(4), 1067 (2006).

[327] T. Yoshiie et al.: Philosophical Magazine A 47(3), 315 (1983). 
[328] I. Yonenaga et al.: Journal of Applied Physics 103(9), 093502 (2008).

[329] H. Van Bueren: Imperfections in Crystals. North-Holland Publishing Company, Amsterdam (1960).

[330] H. M. Zhong et al.: Journal of Applied Physics 99(10), 103905 (2006).

[331] J. Alaria et al.: Journal of Applied Physics 99(8), 08M118 (2006).

[332] C. L. Du et al.: Journal of Applied Physics 99(12), 123515 (2006).

[333] J. Serrano et al.: Physica Status Solidi B 244(5), 1478 (2007).

[334] H. Y. Xu et al.: Journal of Chemical Physics 124(7), 074707 (2006).

[335] C. Bundesmann et al.: Applied Physics Letters 83(10), 1974 (2003).

[336] J. Zuo et al.: Journal of Raman Spectroscopy 32(11), 979 (2001).

[337] F. J. Manjon et al.: Journal of Applied Physics 97(5), 053516 (2005).

[338] C. Sudakar et al.: Journal of Physics 19(2), 026212 (2007).

[339] F. Buciuman et al.: Physical Chemistry Chemical Physics 1(1), 185 (1999).

[340] J. D. Hem et al.: Geochimica Et Cosmochimica Acta 51(6), 1539 (1987).

[341] W. Szuszkiewicz et al.: Acta Physica Polonica A 112(2), 363 (2007).

[342] M. Schumm et al.: Journal of Physics: Conference Series 92, 012149 (2007).

[343] M. H. Sousa et al.: Journal of Raman Spectroscopy 31(3), 185 (2000).

[344] D. deFaria et al.: Journal of Raman Spectroscopy 28(11), 873 (1997).

[345] S. J. Oh et al.: Hyperfine Interactions 112(1-4), 59 (1998).

[346] R. J. THIBEAU et al.: Applied Spectroscopy 32(6), 532 (1978).

[347] H. Labhart: Zeitschrift für Angewandte Mathematik und Physik (ZAMP) 4(1), 1 (1953).

[348] H. J. Kim et al.: Physica Status Solidi B 241(7), 1553 (2004).

[349] D. Hofmann et al.: Physical Review Letters 88(4), 045504 (2002).

[350] K. Vanheusden et al.: Journal of Applied Physics 79(10), 7983 (1996).

[351] R. Kuhnert et al.: Journal of Luminescence 26(1-2), 203 (1981).

[352] J. Sann et al.: Physical Review B 76(19), 195203 (2007).

[353] A. Janotti et al.: Journal of Crystal Growth 287(1), 58 (2006).

[354] Y. M. Sun et al.: Physica B 325(1-4), 157 (2003).

[355] L. Wischmeier et al.: Applied Physics A 84(1-2), 111 (2006).

[356] L. Wischmeier et al.: Physical Review B 74(19), 195333 (2006).

[357] K. Thonke et al.: Physica B 308, 945 (2001).

[358] D. Reynolds et al.: Physical Review B 57(19), 12151 (1998).

[359] C. Klingshirn: Semiconductor Optics. Springer-Verlag (1997). 
[360] D.-C. Park et al.: Applied Surface Science 203-204, 359 (2003).

[361] R. Firestone et al. (editors): Table of Isotopes, volume 1. Wiley \& Sons, New York, 8th edition (1998).

[362] S. Deubler et al.: Nuclear Instruments and Methods in Physics Research Section B 63, 223 (1992).

[363] P. Dahan et al.: Physical Review B 57(16), 9690 (1998).

[364] D. Look et al.: Physical Review Letters 82(12), 2552 (1999).

[365] J. Vechten: Handbook on Semiconductors, volume 3: Materials and Preparation. North-Holland, Amsterdam (1980).

[366] T. Agne et al.: Applied Physics Letters 82(20), 3448 (2003).

[367] Y. Zhang et al.: Journal of Luminescence 85(4), 247 (2000).

[368] P. KOIDL: Physical Review B 15(5), 2493 (1977).

[369] M. A. Reshchikov et al.: Physica B 401, 358 (2007).

[370] C. Klingshirn: ChemPhysChem 8(6), 782 (2007).

[371] N. Y. Garces et al.: Applied Physics Letters 81(4), 622 (2002).

[372] E. G. Bylander: Journal of Applied Physics 49(3), 1188 (1978).

[373] A. Kohan et al.: Physical Review B 61(22), 15019 (2000).

[374] D. Reynolds et al.: Journal of Applied Physics 89(11), 6189 (2001).

[375] P. Erhart et al.: Physical Review B 73(20), 205203 (2006).

[376] C. Van de Walle: Physica B 308-310, 899 (2001).

[377] A. Poppl et al.: Physica Status Solidi A 121(1), 195 (1990).

[378] W. Fowler (editor): Physics of Color Centers. Academic Press, New York (1968).

[379] W. B. FOWLER et al.: Physical Review 128(5), 2154 (1962).

[380] T. Ogino et al.: Japanese Journal of Applied Physics 19(12), 2395 (1980).

[381] S. Shionoya et al.: Journal of The Physical Society of Japan 19(7), 1157 (1964).

[382] D. Pfisterer et al.: Physica B 376, 767 (2006).

[383] T. Frank et al.: Applied Physics A 88(1), 141 (2007).

[384] D. M. Hofmann et al.: Applied Physics A 88(1), 147 (2007).

[385] J. Gambino et al.: Journal of Applied Physics 61(7), 2571 (1987).

[386] Y. Yano et al.: Journal of Materials Research 9(1), 112 (1994).

[387] B. T. Adekore et al.: Journal of Applied Physics 101(2), 024902 (2007).

[388] A. Poppl et al.: Physica Status Solidi A 125(2), 571 (1991).

[389] S. A. Studenikin et al.: Journal of Applied Physics 84(4), 2287 (1998).

[390] H. von Wenckstern et al.: Applied Physics A 88(1), 125 (2007).

[391] I. J. Broser et al.: Solid-State Electronics 21(11-12), 1597 (1978). 
[392] P. J. Dean et al.: Journal of Physics C 14(20), 2847 (1981).

[393] C. West et al.: Physica B \& C 116(1-3), 492 (1983).

[394] M. Deicher: Physica B 389(1), 51 (2007).

[395] M. A. Reshchikov et al.: Journal of Applied Physics 87(7), 3351 (2000).

[396] Q. Li et al.: Europhysics Letters 71(6), 994 (2005).

[397] L. S. Vlasenko et al.: Physical Review B 71(11), 115205 (2005).

[398] P. Thurian et al.: Journal of Crystal Growth 117(1-4), 727 (1992).

[399] D. Look et al.: Solid State Communications 105(6), 399 (1998).

[400] Q. X. Zhao et al.: Applied Physics Letters 87(21), 211912 (2005).

[401] R. O. Kuzian et al.: Physical Review B 74(15), 155201 (2006).

[402] H. J. Kim et al.: Journal of Applied Physics 95(11), 7387 (2004).

[403] J. Schneider: Zeitschrift für Naturforschung Part A A 17(2), 189 (1962).

[404] L. Podlowski et al.: Journal of Luminescence 58(1-6), 252 (1994).

[405] G. A. SLACK et al.: Physical Review 152(1), 376 (1966).

[406] M. A. Valenzuela et al.: Journal Of Photochemistry And Photobiology A-Chemistry 148(1-3), 177 (2002).

[407] S. Geburt et al.: Journal Of Nanoscience And Nanotechnology 8(1), 244 (2008).

[408] S. Geburt: Lanthanoid-dotierte ZnO-Nanodrähte. Master's thesis, Georg-August-Universität Göttingen, II. Physikalischen Institut (2008).

[409] A. B. Djurisic et al.: Small 2(8-9), 944 (2006).

[410] C. Klingshirn et al.: Journal of Luminescence 128(5-6), 792 (2008).

[411] R. Boyn: Physica Status Solidi B 148(1), 11 (1988).

[412] U. Kaufmann et al.: Journal of Physics C 6(2), 310 (1973).

[413] E. Chikoidze et al.: Applied Physics A 88(1), 167 (2007).

[414] J. Schneider et al.: Zeitschrift für Naturforschung Part A A 17(7), 570 (1962). 


\section{Appendix A}

\section{Publications}

List of publications contributing to this thesis:

- C. Borchers, S. Müller, D. Stichtenoth, D. Schwen, and C. Ronning, 'Catalystnanostructure interaction in growth of 1D-ZnO nanostructures', Journal of Physical Chemistry B 110 (2006) 1656-1660

- S. Müller, D. Stichtenoth, M. Uhrmacher, H. Hofsäss, J. Röder, and C. Ronning, 'Unambigiuos identification of the PL-I9-Line in zincoxide', Applied Physics Letters 90 (2007) 011207

- M. Schumm, M. Koerdel, S. Müller, H. Zutz, C. Ronning, J. Stehr, D.M. Hofmann, and J. Geurts, 'Structural impact of Mn implantation on ZnO', New Journal of Physics 10 (2008) 043004

- S. Müller, M. Lorenz, C. Czekalla, H. Hochmuth, G. Benndorf, M. Grundmann, H. Schmidt, and C. Ronning, 'Intense white photoluminescence emission of Vimplanted zinc oxide thin films', Journal of Applied Physics 104 (2008) 123504

- S. Müller, M.J. Zhou, Q. Li, and C. Ronning, 'Intra-shell luminescence of transition metal implanted zinc oxide nanowires', Nanotechnology (2009), accepted

- M. Koerdel, M. Schumm, S. Müller, C. Ronning, Z. Golacki, W. Szuszkiewicz, and J. Geurts, 'Effect of thermal annealing and secondary phase segregation in heavily TM implanted ZnO', Journal of Applied Physics (2009), submitted

Other publications:

- C. Ronning, D. Stichtenoth, and S. Müller, 'Der Draht zur Welt en miniature', Georgia Augusta 4 (2005) 83

- D. Stichtenoth, D. Schwen, S. Müller, C. Borchers, and C. Ronning, 'Optical activation of implanted impurities in ZnS nanowires', Journal of Vacuum Science and Technology A 24 (2006) 1356-1359, selected for: Virtual Journal of Nanoscale Science \& Technology 14 (2006) Issue 1 
- C. Borchers, D. Stichtenoth, S. Müller, D. Schwen, and C. Ronning, 'Catalystnanostructure interaction and growth of ZnS nanobelts', Nanotechnology 17 (2006) 1067-1071

- D. Stichtenoth, D. Schwen, S. Mueller, C. Borchers and C. Ronning, 'Growth and Properties of Zincsulfide Nanowires', in NATO Science Series II, 'Functional Properties of Nanostructured Materials', R. Kassing, P. Petkov, W. Kulisch, and C. Popov (Eds.) 223 (2006), 407-410

- T. Voss, G.T. Svacha, E. Mazur, S. Müller, C. Ronning, D. Konjhodzic, and F. Marlow, 'High order waveguide modes in ZnO nanowires', Nano Letters 7 (2007) 3675

- W. Prost, K. Blekker, Q.-T. Do, I. Regolin, D. Stichtenoth, S. Müller, K. Wegener, C. Ronning, and F.J. Tegude, 'Modeling the carrier mobility in nanowire channel FET'in 'Low-dimesional materials - synthesis, assembly, property scaling, and modeling', M. Shim, M. Kuno, X. Lin, R. Pachter, S. Kumar (eds.), Mat. Res. Soc. Sympo. Proc. Vol. 1017 (2007) DD14-06

- E. Schlenker, A. Bakin, H. Schmid, W. Mader, M. Albrecht, C. Ronning, S. Müller, M. Al-Suleiman, B. Postels, H.H. Wehmann, U. Siegner, and A. Waag, 'Properties of V-implanted ZnO nanorods', Nanotechnology 18 (2007) 125609

- J. Wang, Q.Li, D. Stichtenoth, S. Müller, and C. Ronning, 'Nanomaterial electronic structure investigation by valence electron energy loss spectroscopy - An example of doped ZnO nanowires', Micron 39 (2008) 703

- S. Geburt, D. Stichtenoth, S. Müller, W. Dewald, J. Wang, Y. Jiao, Y.Y. Rao, S.K. Hark, Quan Li, and C. Ronning, 'Rare earth doped ZnO nanowires', Journal of Nanoscience and Nanotechnology 8 (2008) 244-251

- M. Zimmler, J. Bao, F. Capasso, S. Müller, and C. Ronning, 'Laser action in nanowires: Observation of the transition from amplified spontaneous emission to laser oscillation', Applied Physics Letters 93 (2008) 051101

- T. Voss, G.T. Svacha, E. Mazur, S. Müller, and C. Ronning, 'The influence of local heating by nonlinear pulsed laser excitation on the transmission characteristics of a ZnO nanowire waveguide', Nanotechnology 20 (2009) 095702

- D. Höche, S. Müller, M. Shinn, G. Rapin, E. Remdt, M. Gubisch, and P. Schaaf, 'Marangoni convection during free electron laser nitriding of titanium', Metallurgical and Materials Transactions B (2009), accepted

- D. Höche, M. Shinn, S. Müller, and P. Schaaf, 'Diffusion, convection and solidification in cw-mode free electron laser nitrided titanium', Journal of Applied Physics (2009), accepted 


\section{Curriculum vitae}

Persönliche Daten:

Name:

Sven Müller

Geburtstag: $\quad$ 5. August 1980

Geburstort: Eilenburg, Deutschland

Nationalität: deutsch

Familienstand: ledig

Bildungsweg:

1987-1991 Wilhelm-Koenen Oberschule (Grundschule) in Halle Neustadt

1991-1996 Kooperative Gesamtschule Wilhelm von Humboldt (Gymnasium) in Halle

1996-1999 Christian-Weise Gymnasium in Zittau, (Abitur) 1999

2000-2005 Studium der Physik an der Universität Göttingen

Vordiplom 2003

(Note: gut)

Diplom November 2005 ('Wachstum von ZnO Nanodrähten und deren Dotierung durch Ionenstrahlen')

(Note: gut)

2006-2009 Wissenschaftlicher Angestellter, 2. Physikalisches Institut, Universität Göttingen 
A. Publications 


\section{Acknowledgments}

An dieser Stelle möchte ich mich bei allen Personen bedanken, die zum Gelingen dieser Arbeit beigetragen haben.

Beginnen möchte ich mit Prof. Dr. Carsten Ronning. Danke für das sehr weitreichende Thema von Übergangsmetalllegiertem $\mathrm{ZnO}$, deine Motivationskünste auch in den späten Abendstunden, und deine immer offenstehende Tür, auch wenn es am Ende ein weiter Weg zu dir war. Danke, für die Möglichkeit das Thema auf vielen Konferenzen präsentieren zu dürfen und die Forschungsaufenthalte in Hong Kong. Danke für die unermüdlichen Vergleiche zwischen Ost- und Westdeutschland.

Prof. Dr. Hans Hofsäss möchte ich dafür danken, dass ich auch nach Carstens Berufung an die Uni Jena die volle Unterstützung an der Uni Göttingen erhalten habe. Prof. Dr. R. Ulbrich möchte ich für die Bereitschaft diese Arbeit zu begutachten danken.

Einen großen Dank an den Operateur des Ionenbeschleunigers 'Ionas' Detlef Pruschke für die mindestens 50! Tage an denen wir mehr oder weniger erfolgreich versucht haben Ionenstrahlen der verschiedensten Übergangsmetalle zu erzeugen. Natürlich auch für unsere Diskussionen über Gott und die Welt. Ebenso möchte ich mich bei den Werkstätten bedanken, darunter besonders bei meinem Ex-Nachbarn Andreas 'Schnitzel' König. Nicht zuvergessen, Danke an unsere vier guten Feen: Christa, Lucie, Heike und Ina.

Danke an die alte und neue Nanodrahtgruppe für die weitreichenden Diskussionen: Stichte, Geburti, Ralf, Christian, Wilma, Kata, und Joachim.

Danke Hayo, Anne, Uli und der restlichen Arbeitsgruppe von Prof. Hofsäss für die vielen informellen als auch lustigen Gruppenrunden, das Korrekturlesen dieser Arbeit, das gemeisame Grillen oder Chillikochen. Hayo möchte ich nochmal besonders für die FIB Präparation und TEM-Messungen danken.

Ich möchte mich auch bei allen unseren Kooperationspartnern bedanken. Besonders der Zusammenarbeit mit Marcel Schumm, Martin Koerdel, Christian Ziereis und Jean Geurts von der Universität Würzburg für die vielen Ramanmessungen an den Proben und den Diskussionen über die Ergebnisse. Danken möchte ich auch E. Dynowska, Z. Golacki und W. Szuszkiewicz von dem Institute of Physics der Polish Academy of Sciences (Warszawa, Poland) für die vielen Diskussionen über unsere XRD Ergebnisse und die Möglichkeit für die Synchrotron XRD Messungen am DESY in Hamburg. Bedanken möchte ich mich auch bei M. Lorenz, C. Czekalla, 
H. Hochmuth, G. Benndorf und M. Grundmann von der Universität Leipzig und H. Schmidt vom FZ Dresden-Rossendorf für die Bereitstellung der PLD ZnO Schichten und den DLTS Messungen.

Für die große Gastfreundschaft und freundliche Zusammenarbeit möchte ich mich bei Juan Wang, Minjie Zhou und Prof. Quan Li von der Chinese University of Hong Kong bedanken, und für die dort erstellten TEM-Messungen an den Übergangsmetall implantierten ZnO Nanodrähten.

Ich möchte mich bei allen meinen Freunden bedanken, die mir die Zeit während meiner Doktorarbeit versüßt haben. Darunter möchte ich besonders Frank und Corinna für die vielen Reicheltbestellungen, Dokoabende, und abchillen im Gromo danken. Ich möchte mich auch bei Kai, Manuel, Steffe und Krissi für die immer neuen Musikvorschläge und die netten Nächte auf und neben der Tanzfläche bedanken.

Ich möchte meiner Mutter, Schwester und meinen Großeltern dafür danken mich während der gesamten Doktorarbeit mit allen Mitteln unterstütz zuhaben. Ich verspreche in Zukunft wieder öfter vorbeizuschauen. 